\title{
Investigating the role of Porcupine and WNTless: \\ Components of WNT signalling pathway in response to endoplasmic reticulum, oxidative, hypoxia and environmental toxins stresses
}

by

Rowida Mohamed

A thesis submitted to the Faculty of Graduate and Postdoctoral Affairs in partial fulfillment of the requirements for the degree of

\author{
Doctorate \\ in
}

Chemistry

Specialization in Environmental and Chemical Toxicology

Carleton University

Ottawa, Ontario

(C) 2021, Rowida Mohamed 


\section{Abstract}

Porcupine (PORCN) and WNTless (WLS) are factors that control the production of WNT; a protein involved in the early stages of colon cancer development. The WNT proteins play a role in stem cell development and cell differentiation. However, the response of PORCN and WLS to stressors remains to be studied. Previously, we investigated how modifications of PORCN and WLS result in changes in WNT expression and secretion from cells under stress conditions that are found in the tumor microenvironment (hypoxia, oxidative stress, endoplasmic reticulum (ER) stress). The mRNA and protein expression of both PORCN and WLS were notably altered with treatments in human colon cancer (HCT116) cells and human intestinal epithelial (HIEC6) cells. Our results demonstrated significant changes in expression, which led us to investigate the role of different transcription factors during stresses including (GRP78, HIF1 $\alpha$, NFE2L1, NFEL2L2 and MT1). These transcription factors were altered both at levels of the translation and transcription, when overexpressed by PORCN, WLS and WNT3A. WNT and PORCN levels significantly increased at translation and transcriptional levels when overexpressed by PORCN, WLS and WNT3A. WNT and PORCN undergo palmitoyleation; a modification implicated in cancer cell signalling. The inhibition of palmitoyleation by 2-bromohexdecanoic acid (2-BHDA) resulted in a decrease in the modified forms of PORCN in HCT116 cells. Furthermore, the data showed that palmitoyleation plays an essential role in the expression of WNT proteins; specifically, inhibiting WNT3A and decreasing WLS protein expression in HCT116 cells. However, WLS and WNT3A are modified by N-linked glycosylation. Emerging evidence indicates the critical role of glycosylation in tumor aggressiveness, progression, and metastasis. Cell 
lysates from hypoxic HCT116 cells, containing induced WLS and WNT3A proteins, HCT116 cells were subjected for deglycosylation. The production of glycosylated WLS and WNT3A was inhibited. We also investigated the role of cadmium in activating WNT signalling and its components and our results showed a significant increase of the WNT proteins, and the transcription factors, when exposed to dose-specific concentration of cadmium at $5 \mu \mathrm{M}$. These notable findings show the essential role of post-translation modification of PORCN and WLS and how it alters WNT signalling during stress conditions. 


\section{Acknowledgements}

I feel extremely privileged to be surrounded by a group of supportive and encouraging people who have greatly contributed to my professional development and growth. First and foremost, I want to thank my thesis advisor and mentor Dr. William Willmore for his unwavering support and contagious enthusiasm throughout these five years. He has been an integral part of my learning process, and this work would have never been possible without his devoted guidance and scientific insight. Dr. Willmore's passion and positive attitude towards research and teaching science has encouraged my academic and personal journey. A great amount of his valuable time was used to give me constructive suggestion to complete my work. I appreciate his warm and gentle approach to any issues that occurred with the data. Through our interactions I have learnt so much, not just about science but also about life! Thank you for pushing me to achieve more than I ever thought I was capable of, and for giving me confidence in both my science and myself. Working with you and the lab has been the best experience of my life. I hold the upmost gratitude for giving the opportunity to complete my doctoral training in your lab; it has been a great journey.

I would like to thank my committee members for taking the time and effort to read to my thesis and be present and interested in my topic.

Special thanks to my colleague and very good friend Shana Cameron. You have been a positive and an awesome friend.

I would like to thank all former and current members of the Willmore Lab: Shana, Anand, Hayiun, Matthew, Marwa, Jessica, Jacob, Mary, Nekha, Ramak, Tombi, Tristan, James, Babur, Alison, Emily, and Florian. 
I would like to extend my thanks to my department. The lab coordinators Mastaneh Azad, Elan and Peter Mosher. The grad office coordinator Chantal Gravelle. My comprehensive committee members, Drs. Wang Wong, Sean Barry, Apollinaire Tsopmo, David McMullin and Anatoli Ianoul.

I would like to thank Dr. Farah Hosseinian for being a mentor and a great role model, being both encouraging and supportive.

I would like to acknowledge and thank Sonia Tangauy, PMC Coordinator, and all the PMC staff: Laurie, Maureen Jones, Bruce Hamm, Larry McCloskey, and all the exam center stuff at PMC.

Nancy, Tina and David working in Environmental Health and Safety at Carleton University. Thank you for giving me the opportunity and support through my student work experience.

Thanks to everyone who, in one way or another, contributed to the completion of this work. Thanks to Dr. Bruce McKay and his imager and lab equipment and the EVOS microscope from Dr. Jenny Bruin's lab.

Thanks to Dr. Apolliaire Tsopmo, awesome mentor, and great support through all my Comprehensive Exams and Masters degree. You have been a great support to my success and very encouraging mentor.

I would like to thank my family for their continuous encouragement from all my sisters and my mother. I dedicate my thesis to my loving and caring mother Salwa Abdalla. My mother not only raised and nurtured me and my sisters, she taxed herself dearly over the years for my education and intellectual development. I hope by completing this thesis I can make you proud. 


\section{Table of Contents}

Abstract 2

$\begin{array}{ll}\text { Acknowledgements } & 4\end{array}$

$\begin{array}{ll}\text { Table of Contents } & 6\end{array}$

List of Tables 13

List of Illustrations 14

Chapter 1: Introduction 1

1.1 Colon Cancer 1

1.2 The emerging roles of WNT in human colon cancer 4

$\begin{array}{llr}1.3 & \text { WNT signalling pathway } & 8\end{array}$

1.4 Functions of WNT signalling network 9

$\begin{array}{lll}1.5 & \text { The WNT ligands } & 12\end{array}$

$\begin{array}{lll}\text { 1.6 WNT signaling pathways } & 15\end{array}$

$\begin{array}{lll}\text { 1.6.1 Canonical WNT/ } \beta \text {-catenin-dependent } & 15\end{array}$

$\begin{array}{ll}\text { 1.6.2 Non-canonical WNT pathway } & 17\end{array}$

$\begin{array}{lll}\text { 1.6.2.1 WNT/calcium pathway } & 18\end{array}$

$\begin{array}{lll}\text { 1.6.2.2 WNT/PCP pathway } & 18\end{array}$

1.7 The WNT secretory route and modification 21

$\begin{array}{lll}\text { 1.7.1 Porcupine (PORCN) } & 23\end{array}$ 
1.7.2 Palmitoyleation of WNT 26

1.7.3 Evi-WNTLESS (WLS) the WNT chaperone 31

1.7.4 Glycosylation of WNT 36

1.8 The tumor microenvironment 40

$\begin{array}{lll}\text { 1.8.1 Endoplasmic reticulum stress } & 40\end{array}$

1.8.1.1 WNT secretion and endoplasmic reticulum stressors 41

$\begin{array}{lll}\text { 1.8.2 Hypoxia } & 42\end{array}$

1.8.2.1 WNT secretion and hypoxia 44

$\begin{array}{lll}\text { 1.8.3 Oxidative stress } & 45\end{array}$

1.8.3.1 WNT secretion and oxidative stress 46

$1.9 \quad$ Stress induced transcriptional activation factors 50

1.9.1 Glucose-regulated protein 78 (GRP78) 52

1.9.2 Nuclear factor erythroid-1 and 2-related factor (NFE2L1 or Nrf2) 52

1.10 Hypothesis and objectives $\quad 54$

$\begin{array}{ll}\text { Chapter 2: Materials and Methods } & 59\end{array}$

$\begin{array}{lll}2.1 & \text { Cell culture } & 59\end{array}$

2.2 Assessment of total cell viability 59

$\begin{array}{lll}2.3 & \text { Chemicals } & 60\end{array}$

2.4 Construction of plasmids and gene cloning 61 
2.5 Cellular reactive oxygen species (ROS with DCFDA)

$\begin{array}{lll}2.6 & \text { Transfections } & 62\end{array}$

2.7 Hypoxia and hypoxia mimetic treatments 63

2.8 Gene expression by quantitative polymerase chain reaction (qPCR) 64

2.9 Membrane-bound protein extraction $\quad 64$

2.10 Gel electrophoresis and immunoblotting analysis 65

2.11 Promoter analysis and electrophoretic mobility shift assay 66

2.11.1 Preparation of whole cell extracts 68

$\begin{array}{lll}2.11 .2 & \text { Probe synthesis } & 68\end{array}$

$\begin{array}{ll}2.11 .3 \text { Binding reactions } & 68\end{array}$

2.12 Knockdown of PORCN and WLS using siRNA 69

2.13 Inhibition of WNT post-translation modification in the secretory route 70

$\begin{array}{lll}\text { 2.13.1 PNGase F assay (inhibition of glycosylation) } & 70\end{array}$

2.13.2 Inhibition of palimatoylation using 2-bromohexadecanoic acid (2BHDA) $\begin{array}{ll}\text { and Stearoyl-CoA-Desaturase 1 (SCD1) inhibitor } & 71\end{array}$

$\begin{array}{lll}2.14 & \text { Statistical analysis } & 72\end{array}$

Chapter 3: Responses of Porcupine and WNTless proteins to oxidative, hypoxic and $\begin{array}{ll}\text { endoplasmic reticulum stresses } & 73\end{array}$

$\begin{array}{lll}3.1 & \text { Introduction } & 73\end{array}$

$\begin{array}{lll}3.2 & \text { Results } & 76\end{array}$ 
3.2.2 The effect of hypoxia and hypoxia mimetics condition on the gene expression of PORCN and WLS in HCT116 cells

3.2.3 Effect of physicochemical stressors on the gene expression of PORCN and WLS on HCT116 cells

3.2.4 PORCN and WLS response are activated upon exposure to ER stressors 80

3.2.5 Hypoxia and hypoxic mimetics lead to elevated and modified protein expression in PORCN and WLS

3.2.6 Electrophoretic mobility shift assay (EMSA) shows that PORCN and WLS genes under hypoxia, hypoxia mimetics, ER stressors and oxidative stressors induces the activation of $\mathrm{HIF} 1 \alpha, \mathrm{NRF} 2$ and NF- $\kappa \mathrm{B}$ activation

3.2.7 Knockdown of PORCN and WLS using siRNA

3.8.2 Inhibition of palimatoylation using 2-bromohexadecanoic acid (2BHDA) 92

3.8.3 PNGase F assay (deglycosylation of WNT proteins)

3.8.4 WNT3A response are activated upon exposure to hypoxia and hypoxia mimics, oxidative and ER stressors

3.8.4.1 The effect of hypoxic conditions on gene and protein expression of PORCN, WLS and $\beta$-catenin in vitro 
3.8.4.2 The effect of ER and oxidative stressors on gene and protein expression

of PORCN, WLS, WNT3A and $\beta$-catenin in vitro

104

3.8.4.3 The effect of PORCN and WLS knock down on PORCN, WLS and

WNT3A protein expression in vitro

3.8.4.4 The effects of PORCN and WLS knock down on WNT3A expression 108

3.8.4.5 Palmitoyleation is necessarily for WNT secrection

109

3.4 Conclusions

Chapter 4: Transcription factor control of WNT signalling pathway components 111

4.1 Introduction

4.2 Results

4.2.1 Effect of SCD1 and 2BHDA on the cell viability

4.2.2 Gene expression of transcription factors when treated with SCD1

inhibitor and inhibition of palmitoyleation

4.2.3 Gene expression of WNT-associated genes with 2BHDA and SCD1 inhibitor treatments

4.2.4 Expression profile of GRP78, HIF1, NFE2L1 and NFE2L2 genes when treated with hypoxia, ER and oxidative stress.

4.2.5 Relative $\beta$-catenin expression with overexpression of PORCN, WLS and WNT3A 
4.1.1 Palmitoyleations and stearoyl CoA desaturases genes regulates GRP78, HIF $1 \alpha, N F E 2 L 1$ and NFE2L2

4.1.2 Stearoyl CoA desaturase is required to produce active, lipid-modified WNT proteins

4.1.3 Treatment with ER, hypoxia and oxidative stresses elevate the gene expression of GRP78, HIF1 alpha, NFE2L1 and NFE2L2 in HIEC-6 cells

4.1.4 Overexpression of PORCN, WLS, and WNT3A induces levels of GRP78, HIF1 $\alpha$, NFE2L1, and NFE2L2 and $\beta$-catenin protein expression

Chapter 5: Cadmium induced toxicity on WNT proteins and transcription factors 139

5.1 Introduction

5.2.1 Effect of cadmium chloride on cell viability

5.2.2 Cadmium exposure induced ROS using (DCFDA, also known as $\mathrm{H}_{2}$ DCFDA).

5.2.3 Expression profile of transcription factors in cadmium-treated cells

5.2.4 Induction of WNT gene expression in cadmium-treated cells 
5.3.1 Induction of ROS by cadmium chloride

5.3.2 Cadmium altered WNT gene and protein expression

5.3.3 Effect of cadmium on transcription factors

5.4 Conclusions

Table 2: List of all the primers used in all the experiments (Cloning, qPCR, EMSA, siRNA). 


\section{List of Tables}

Table 1: List of mammalians WNTS and their functions. The WNT proteins contain 19 different family members of WNT and are essential secretory factors involved in WNT pathways (canonical and non-canonical).

Table 2: list of all the primers used in all the experiments (cloning, qPCR, EMSA, siRNA) in Appendices

228-229 


\section{List of Illustrations}

Figure 1.1: Estimated age-standardized incidence rate (standard: world population) of colorectal cancer in 2012 (source: Globocan 2012, International Agency for Research on Cancer).

Figure 1.2: WNT signalling affects the function and structure of the colon tissue. WNT signalling with mutant APC is the main cause of colon cancer. The image on the left illustrates WNT signaling in its active form, with the continuous degradation of $\beta$-catenin. The middle image shows the condition when APC is deleted, which affects the activity of WNT signalling. The image on the right shows' inhibitors of WNT signalling in Apcmutant cells. B, immunohistochemistry for $\beta$-catenin in a human colorectal carcinoma showing increased nuclear localization as a surrogate marker of active WNT in the invasive front compared with the tumor center (Dustin et al., 2019).

6-7

Figure 1.3: The role and function of WNT signaling in different organs and tissues in the human body (image created by R. Mohamed using Biorender). The role of WNT can be seen in every part of the human body, and specifically the vital organs (brain, liver, breast, reproductive system, gastrointestinal tract, and bone).

Figure 1.4: Schematic overview of WNT signaling pathways (a) WNT-OFF: in the canonical WNT pathway, the absence of WNT signaling leads to proteasome degradation of $\beta$-catenin that mediate destruction complex including several proteins (GSK-3 $\beta$ and CK1 $1 \alpha$ kinases, result in $\beta$-catenin phosphorylation). (b) WNT-ON: the activation of WNT 
signaling start of the WNT ligand binding to its Fz receptor to LRP5/6 co-receptor to the initiate DVL together with the destruction complex to the cell membrane. However, the destruction box that includes GSK-3 $\beta$ and CK1 $1 \alpha$ mediated phosphorylation of $\beta$-catenin, is inhibited and the stabilized $\beta$-catenin is translocated to the nucleus where it serves as coactivator for Tcf/LEF transcription factors. (c) Non-canonical pathway $\mathrm{WNT} / \mathrm{Ca}^{2+}$, the interaction of WNT ligands to a specific receptor activates the PLC, initiate intracellular $\mathrm{Ca}^{2+}$ release result in activation of PKC and CaMKII that regulate the activation of NF-AT transcription factor and gene expression. (d) In the non-canonical WNT/PCP signaling pathway, the binding of WNT ligand to the receptor triggers a signalling cascade that involves small Rho-family GTPases and downstream effectors such as JNK and ROCK, which in turn modulate cytoskeleton organization and gene expression (Qu et al., 2019).

Figure 1.5: Showing the upstream pathway of WNT signalling, highlighting all the essential components of WNT secretions. Upon translation, WNT proteins undergo a series of modifications as they transit through the secretory pathway and associate with several proteins, including Porcupine (PORCN) in the endoplasmic reticulum. All WNT proteins harbor a covalent lipid modification: a palmitoleic acid, attached by the palmitoyltransferase PORCN. The transmembrane protein WNTless (WLS) binds exclusively to lipidated WNT proteins and transfers them to the plasma membrane for secretion. (Gonzalez-Fernandez et al., 2016a). 
Figure 1.6: Domain organization of PORCN. The three-dimensional structure of all three PORCN is not known (a) PORCN isoforms are approximately $51 \mathrm{kDa}$ and palmitoyleation sites (yellow). It has nine transmembrane (TM) domains (gold). There are four isoforms of PORCN (A, B, C and D) that differ by 5-11 amino acids within the third cytoplasmic loop (shown in insert). It has two sites of palmitoylation (yellow), and the carboxy-terminal tail that is predicted to be embedded within the phospholipid bilayer. The TM domains are defined by the following amino acids: $29-47,67-89,96-115,155-177,197-219,250-$ 271, 343-360, and 395-417.

Figure 1.7: A schematic model illustration how WNT gets palimtoyleated by Porcupine (PORCN). PORCN is palmitoyleated at residue Cys187 Lipid modification of PORCN then regulates the acylation level of WNT3A. PORCN is fatty acylated via a thioester linkage. PORCN recognizes fatty acid Coenzyme A (CoA) substrates that have 13-16 carbon atoms including palmitoleoyl-CoA and acylates newly synthesized WNT3A in the ER at conserved Ser209. Cys77 residues on WNT3A are not lipid modified. PORCN fatty acylation (at Cys187) adjusts the level of WNT3A palmitoyleation and its signaling activity. The O-palmitate bound to WNT3A does not undergo rapid cycles of acylation and deacylation during the lifetime of the protein. Palmitoyleation of WNT3A neither requires glycosylation nor protects WNT3A from degradation in the proteasome, and it is required for maximal WNT3A secretion. Monoglycosylation of palmitoylated WNT3A occurs in the ER-Golgi system and sets it routes to secretion. Mature WNT3A traffics post-Golgi which act as export sites for its secretion on exosomes. PAT, palmitoyl acyltransferase 
(adapted from Rios-Esteves and Resh, 2014 using ChemDraw tool by R. Mohamed).

Figure 1.8: WLS is a $62-\mathrm{kDa}$ protein that is proposed to have seven or eight TM domains (gold) and one glycosylated site between site 1 (blue) and 2 (green) (adapted from Nygaard et al. (2020))

Figure 1.9: The structure of WNT8A bound to WLS, with WLS colored in rainbow as in (D) and WNT8A in violet with a transparent surface. The PAM is represented as green spheres extending out between TM helices 4 and 5. Glycosylation of WNT8A at two sites is shown in red, represented as sticks (Nygaard et al., 2020).

Figure 1.10: Illustration showing the WNT ligand gets chaperoned by protein WLS. WLS is an eight transmembrane protein. WLS N-glycosylation will increase the affinity of WNT binding, allowing WNT stabilization and enhance WNT secretion to activate WNT signaling pathway (adapted from Li and Niswander, 2020).

Figure 1.11: Illustration of the sources of stressors and their effect on the balance of antioxidant system. Exogenous and endogenous sources of stressors could result in an increase in ROS which lead to deregulation of cellular signaling will results in chronic illness and cancer. 
Figure 1.12: Illustrates the crucial role of UPR in all the human tissues and organs could results from ER, hypoxia and oxidative stressors. UPR effect wide range of physiological and pathological outcomes in many chronic illnesses and diseases. http://www.trainers.eu/background/.

Figure 1.13: Schematic diagram of potential of WNT pathways linked to hypoxia, ER and oxidative stressors which controls and regulates WNT secretion. In the secreting cells the WNT ligand is translated into the endoplasmic reticulum (ER) where it is bound and modified with lipids by the membrane-bound-O-acyltransferase PORCN. The lipidated WNT is transported by a yet unknown mechanism to the Golgi. The transmembrane protein WLS binds to WNT in the Golgi and escorts it to the plasma membrane. From there WNTs are eventually released into the extracellular space. In the WNT-expressing cells WLS is saved from lysosomal degradation by binding to the retromer complex and is retrieved to the trans-Golgi for additional rounds of WNT secretion. This leads to cytoplasmic stabilisation and subsequent nuclear translocation of $\beta$-catenin. In the nucleus $\beta$-catenin associates with transcriptional coactivators and target genes are transcribed. Endoplasmic Reticulum (ER) stress (also known as the unfolded protein response (UPR)) is a concurrent stress found in many stress conditions (hypoxia, oxidative stress, toxin stress). During ER stress, essential proteins released from IRE1 $\alpha$, PERK and ATF6 sensors allowing their dimerization/oligomerization export to the Golgi apparatus. PERK activation leads to phosphorylation of NRF2, HIF1 $\alpha$ and eIF2 $\alpha$ as well as the expression of genes involved in antioxidant response, protein folding, and apoptosis. Hypoxia induces the activation of HIF proteins and WNT signaling (Boso et al., 2019).

57-58 
Figure 2.1: Promoter analysis of (a) PORCN and (b) $W L S$ genes. Analysis of the PORCN and $W L S$ promoters was performed using the ExPASy Bioinformatics Resources Portal. Antioxidant Response Elements (AREs), Hypoxia Response Elements (HREs) and NF-kBbinding sites were identified and further tested with Electrophoretic Mobility Shift Assays (EMSAs) using promoter-specific sequences.

Figure 3.1: Graphical abstract showing how hypoxia, oxidative, and ER stressors potentially cross-talk with the WNT secretory pathway. The illustration displays modification of PORCN (palmitoylation) and WLS (glycosylation) could altered as a result of physiological and chemical stresses. Moreover, these changes possibly affect the binding of WNT to the receptors FZ/LPR5/6 (image created by Dr. William Willmore using BioRender).

Figure 3.2: The effect of hypoxia and hypoxic mimetics (a and b), ER stressors and oxidative stressors ( $\mathrm{c}$ and d) on PORCN and WLS gene expression in human colorectal carcinoma (HCT116) cells. HCT116 cells were treated with hypoxia $\left(1 \% \mathrm{O}_{2}\right.$ and $\left.5 \% \mathrm{CO}_{2}\right)$ and hypoxic mimetics (100 $\mu \mathrm{M}$ desferrioxamine (DFO) and $1 \mathrm{mM}$ dimethyloxalylglycine (DMOG), (5 mg/mL tunicamycin (Tuni), $3 \mathrm{nM}$ thapsigargin (Thap), $90 \mathrm{mM}$ antimycin $\mathrm{A}$ (AA), $2 \mathrm{M}$ dithiothreitol (DTT) for $24 \mathrm{~h}$ and $5 \mathrm{mg} / \mathrm{mL}$ MG132 for $3.5 \mathrm{~h}$. Cells without treatment were used as controls. Total RNA was isolated from HCT116 cells, converted to cDNA and analyzed by qPCR using gene- specific primers for a and $\mathrm{c}(\mathrm{PORCN})$ and $\mathrm{b}$ and d (WLS) and target genes. The level of target genes of each mRNA was normalized to the GAPDH mRNA. Data are the means from three independent experiments \pm S.E.M. Data 
analysis was done using one-way ANOVA followed by Dunnett's test; *p $<0.05$, ** $p$ $<0.01, * * * \mathrm{p}<0.001$

Figure 3.3: Western blot showing the effects of 24 hours of treatment on HIEC-6 and HCT116 protein expression. Cultured HIEC-6 and HCT116 cells were treated with hypoxia $\left(1 \% \mathrm{O}_{2}\right.$ and $5 \% \mathrm{CO}_{2}$ ), hypoxic mimetics (100 $\mu \mathrm{M}$ desferrioxamine (DFO), $1 \mathrm{mM}$ dimethyloxalylglycine (DMOG)), and ER stressors ( $5 \mathrm{mg} / \mathrm{mL}$ tunicamycin (Tuni), $3 \mathrm{nM}$ thapisigargin (Thap), $90 \mathrm{mM}$ antimycin A (AA), $2 \mathrm{M}$ dithiothreitol (DTT)) for $24 \mathrm{hr}$ and 5 $\mathrm{mg} / \mathrm{mL}$ MG132 for $3.5 \mathrm{hr}$. Cells without treatment were used as controls. Total membrane bound proteins were isolated using $8 \mathrm{M}$ urea. Samples were incubated with anti-WLS (65 $\mathrm{kDa})$, anti-PORCN (55 kDa) or anti- $\beta$-catenin $(92 \mathrm{kDa})$ antibodies. The level of protein expression of each sample was normalized to $\beta$-tubulin. Data are the average band densities from three independent experiment \pm S.E.M. Data analysis was done using one-way ANOVA followed by Dunnett's test; ${ }^{*} \mathrm{p}<0.05,{ }^{* *} \mathrm{p}<0.01,{ }^{* * *} \mathrm{p}<0.001$.

Figure 3.4: Effects of hypoxia, hypoxic mimetics (DMOG, DFO, MG132), ER stressors (Tuni, Thap, DTT, MG132) and oxidative stressors (AA) on HIF-1, Nrf2 and NF-кB DNA binding to PORCN and $W L S$ promoter elements (a, b, c and d). Whole cell extracts were prepared and EMSA carried out of HIF-1 using HRE (consensus site) (a and b), Nrf2 using ARE (c) and NF- $\mathrm{kB}$ (d) labelled oligonucleotides corresponding to the consensus sites in PORCN and WLS promoter. Cells were overexpressed by PORC (a and d) and WLS (b and c) for $24 \mathrm{hrs}$. Overexpressed cells were treated with hypoxia $\left(1 \% \mathrm{O}_{2}\right.$ and $\left.5 \% \mathrm{CO}_{2}\right)$, hypoxic mimetics (100 $\mu \mathrm{M}$ desferrioxamine (DFO), $1 \mathrm{mM}$ dimethyloxalylglycine 
(DMOG)), and ER stressors (5 mg/mL tunicamycin (Tuni), $3 \mathrm{nM}$ thapisigargin (Thap), 90 $\mathrm{mM}$ antimycin A (AA), $2 \mathrm{M}$ dithiothreitol (DTT)) for $24 \mathrm{hr}$ and $5 \mathrm{mg} / \mathrm{mL}$ MG132 for 3.5 hr. Cells without treatment were used as controls. Data are the average band densities from three independent experiment \pm S.E.M. Data analysis was done using one-way ANOVA followed by Dunnett's test; *p $<0.05,{ }^{* *} \mathrm{p}<0.01,{ }^{* * *} \mathrm{p}<0.001$.

Figure 3.5: The siRNA knock down of $P O R C N$ and $W L S$ genes were detected by Western blot analysis. HCT116 cells were transfected with PORCN and WLS siRNA and Negative Control siRNA, and the levels of PORCN, WLS and WNT3A protein were detected by Western blot. The quantitative comparison of the difference of expression of PORCN, WLS, and WNT3A in each group. Total protein levels were normalized to levels of $\beta$ tubulin protein. The data are presented as the means \pm S.E.M. from at least 3 independent experiments $(* \mathrm{p}<0.05 ; * * \mathrm{p}<0.01 ; * * * \mathrm{p}<0.001$ by Dunnett's test).

90-91

Figure 3.6: Effects of PORCN ( $\left.\mathrm{P}_{\mathrm{KD}}\right)$ and WLS ( $\left.\mathrm{W}_{\mathrm{KD}}\right)$ knock down and inhibition of palmitoyleation by 2-bromohexadecanoic acid (2BHDA) on the expression PORCN, WLS and WNT3A in HCT116 cells. Cells were transfected using siRNA targeting PORCN and WLS under normoxic and hypoxic (1\% oxygen) conditions.

Figure 3.7: Effects of PORCN ( $\left.\mathrm{P}_{\mathrm{KD}}\right)$ and WLS ( $\left.\mathrm{W}_{\mathrm{KD}}\right)$ knock down and inhibition of Nglycosylation by F-PGNase on the expression PORCN, WLS, and WNT3A in HCT116 cells. Cells were transfected using siRNA targeting PORCN and WLS under normoxic and hypoxic (1\% oxygen) conditions. 95 
Figure 3.8: Western blot showing the effects of 24 hours of treatment on HIEC-6 and HCT116 protein expression. Cultured HIEC-6 and HCT116 cells were treated with hypoxia $\left(1 \% \mathrm{O}_{2}\right.$ and $\left.5 \% \mathrm{CO}_{2}\right)$, hypoxic mimetics (100 $\mu \mathrm{M}$ desferrioxamine (DFO), $1 \mathrm{mM}$ dimethyloxalylglycine (DMOG)), and ER stressors (5 mg/mL tunicamycin (Tuni), $3 \mathrm{nM}$ thapisigargin (Thap), $90 \mathrm{mM}$ antimycin A (AA), $2 \mathrm{M}$ dithiothreitol (DTT)) for $24 \mathrm{hrs}$ and $5 \mathrm{mg} / \mathrm{mL}$ MG132 for $3.5 \mathrm{hrs}$. Cells without treatment were used as controls. Total membrane bound proteins were isolated using $8 \mathrm{M}$ urea. Samples were incubated with antiWNT3A (45 kDa) antibodies. The level of protein expression of each sample was normalized to $\beta$-tubulin. Data are the average band densities from three independent experiment \pm S.E.M. Data analysis was done using one-way ANOVA followed by Dunnett's test; ${ }^{*} \mathrm{p}<0.05, * * \mathrm{p}<0.01, * * * \mathrm{p}<0.001$.

Figure 3.9: The effect of hypoxia and hypoxic mimetics, ER stressors and oxidative stressors on WNT3A gene expression in human colorectal carcinoma (HCT116) cells. HCT116 cells were treated with hypoxia $\left(1 \% \mathrm{O}_{2}\right.$ and $\left.5 \% \mathrm{CO}_{2}\right)$ and hypoxic mimetics $(100$ $\mu \mathrm{M}$ desferrioxamine (DFO) and $1 \mathrm{mM}$ dimethyloxalylglycine (DMOG), $(5 \mathrm{mg} / \mathrm{mL}$ tunicamycin (Tuni), $3 \mathrm{nM}$ thapsigargin (Thap), $90 \mathrm{mM}$ antimycin $\mathrm{A}$ (AA), $2 \mathrm{M}$ dithiothreitol (DTT) for $24 \mathrm{hr}$ and $5 \mathrm{mg} / \mathrm{mL}$ MG132 for $3.5 \mathrm{hr}$. Cells without treatment were used as controls. Total RNA was isolated from HCT116 cells, converted to cDNA, and analyzed by qPCR using gene-specific primers for WNT3A. The level of target genes of each mRNA was normalized to the GAPDH mRNA. Primers used for WNT3A gene expression were 5'-GACTTCCTCAAGGACAAGTACG-3' (forward) and 5'- 
GGAACCTTGAAGTAGGTGTAG-3' (reverse). Data are the average from three independent experiments \pm S.E.M. Data analysis was done using one-way ANOVA followed by Dunnett's test; *p $<0.05$.

98-99

Figure 4.1: Cell viability assay. The viability of HIEC-6 cells, in exponential stage of growth, after $24 \mathrm{hrs}$ of exposure to a) SC1 inhibitor and b) 2BHDA as measured by the MTT (3-(4,5-dimethylthiazol-2-yl)-2,5-diphenyltetrazolium bromide) assay.

115

Figure 4.2: The effect of 2BHDA and SCD1 inhibitor on GRP78, HIF1 $\alpha, N F E 2 L 1$ and NFE2L2 gene expression in human intestinal epithelium cell 6 (HIEC-6) cells. HIEC-6 cells were treated with $100 \mu \mathrm{M}$ of 2BHDA and $10 \mu \mathrm{M}$ of SCD1 inhibitor (24 hrs). Cells without treatment were used as controls. Total RNA was isolated from HCT116 cells, converted to cDNA and analyzed by qPCR using gene-specific primers for a $(G R P 78), \mathrm{b}$ (HIF 1 $\alpha), \mathrm{c}(N F E 2 L 1)$ and $\mathrm{d}(N F E 2 L 2)$ and target genes. The level of target genes of each mRNA was normalized to the GAPDH mRNA. Data show the average from three independent experiments \pm S.E.M. Data analysis was done using one-way ANOVA followed by Dunnett's test; ${ }^{*} \mathrm{p}<0.05, * * \mathrm{p}<0.01, * * * \mathrm{p}<0.001$.

117-118

Figure 4.3: The effect of 2BHDA and SCD1 inhibitor on PORCN, WLS1 $\alpha, W N T 3 A$ and WNT5A gene expression in human intestinal epithelium cell 6 (HIEC-6) cells. HIEC-6 cells were treated with $100 \mu \mathrm{M}$ of 2BHDA and $10 \mu \mathrm{M}$ of SCD1 inhibitor (24 hrs). Cells without treatment were used as controls. Total RNA was isolated from HCT116 cells, converted to cDNA and analyzed by qPCR using gene-specific primers for a $(P O R C N), \mathrm{b}(W L S)$, 
$(W N T 3 A)$ and $\mathrm{d}(W N T 5 A)$ and target genes. The level of target genes of each mRNA was normalized to the GAPDH mRNA. Data show the average from three independent experiments \pm S.E.M. Data analysis was done using one-way ANOVA followed by Dunnett's test; ${ }^{*} \mathrm{p}<0.05,{ }^{* *} \mathrm{p}<0.01, * * * \mathrm{p}<0.001$.

120-121

Figure 4.4: The effect of hypoxia and hypoxic mimetics, ER stressors and oxidative stressors on GRP78, HIF1 $\alpha$, NFE2L1 and NFE2L2 gene expression in Human Intestinal Epithelial Cells (HIEC-6). HIEC-6 cells were treated with hypoxia $\left(1 \% \mathrm{O}_{2}\right.$ and $\left.5 \% \mathrm{CO}_{2}\right)$ and hypoxic mimetics (100 $\mu \mathrm{M}$ desferrioxamine (DFO) and $1 \mathrm{mM}$ dimethyloxalylglycine (DMOG), (5 mg/mL tunicamycin (Tuni), $3 \mathrm{nM}$ thapsigargin (Thap), $90 \mathrm{mM}$ antimycin $\mathrm{A}$ (AA), $2 \mathrm{M}$ dithiothreitol (DTT) for $24 \mathrm{hrs}$ and $5 \mathrm{mg} / \mathrm{mL}$ (MG132) for $3.5 \mathrm{hrs}$. Cells without treatment were used as controls. Total RNA was isolated from HIEC-6 cells, converted to cDNA and analyzed by qPCR using gene-specific primers for a) GRP78, b) HIFl $\alpha$, c) NFE2L1 and d) NFE2L2 and target genes. The level of target genes of each mRNA was normalized to the GAPDH mRNA. Data show the average from three independent experiments \pm S.E.M. Data analysis was done using one-way ANOVA followed by Dunnett's test; ${ }^{*} \mathrm{p}<0.05,{ }^{* *} \mathrm{p}<0.01,{ }^{* * *} \mathrm{p}<0.001$.

123-124

Figure 4.5: PORCN, WLS, and WNT3A were overexpressed using Oligofectamine in (a) HCT116 and (b) HIEC-6) and MG132 were used to treat overexpressed PORCN, WLS, and WNT3A in HCT116. The Western blotting analysis showed that PORCN, WLS, and WNT3A-OE promoted the levels of $\beta$-catenin related proteins expression. The level of protein expression of each sample was normalized to $\beta$-tubulin. Data are the average band 
densities from three independent experiment \pm S.E.M. Data analysis was done using oneway ANOVA followed by Dunnett's test; ${ }^{*} p<0.05,{ }^{* *} \mathrm{p}<0.01,{ }^{* * *} \mathrm{p}<0.001$.

126-127

Figure 4.6: PORCN, WLS, and WNT3A were overexpressed using Oligofectamine in human epithelium intestinal cells (HIEC-6) and MG132 was used to treat overexpressed HIEC-6. (a) The Western blotting analysis showed that PORCN, WLS WNT3A-OE promoted the levels of GRP78-, HIF1 $\alpha$-, NFE2L1-, and NFEL2-related proteins expression. Panels (b), (c) and (d) corresponding densitometric quantification of protein expression. The level of protein expression of each sample was normalized to $\beta$-tubulin. Data are the average band densities from three independent experiment \pm S.E.M. Data analysis was done using one-way ANOVA followed by Dunnett's test; *p $<0.05, * *$ $\mathrm{p}<0.01, * * * \mathrm{p}<0.001$.

129-130

Figure 4.7: PORCN, WLS, and WNT3A were overexpressed using oligofectamin in human colon cancer adenocarcinoma cell (HCT116) and MG132 was used to treat overexpressed HCT116. a) The Western blotting analysis showed that PORCN, WLS WNT3A-OE promoted the levels of GRP78, HIF1 $\alpha$, NFE2L1, and NFEL2 related proteins expression. b) Panels (b), (c), and (d) correspond to the densitometric quantification of protein expression. The level of protein expression of each sample was normalized to $\beta$ tubulin. Data are the average band densities from three independent experiment \pm S.E.M. Data analysis was done using one-way ANOVA followed by Dunnett's test; *p $<0.05, * *$ $\mathrm{p}<0.01, * * * \mathrm{p}<0.001$ 131-132 
Figure 5.1: Cytotoxic effect of cadmium chloride on HCT116 and HIEC-6 cells. Cells were cultured with increasing concentrations $(0,2.5,5,7.5,10$ and $12.5 \mu \mathrm{M})$ of cadmium chloride for 24 hrs. Cell viability was determined based on the MTT assay. Each point represents a mean \pm S.E.M of three experiments with three replicates per concentrations.

144-145

Figure 5.2: Represent the effect of cadmium treated cells at different concentrations in HIEC-6 and HCT116. The cells were then stained with $\mathrm{DCFH}_{2}$-DA. Panels show ROS generation in HIEC-6 and HCT116 treated at $0,2.5,5$ and $10 \mu \mathrm{M}$ of $\mathrm{CdCl}_{2}$. The images illustrated an increase in cell death at the concentration of cadmium increased. Moreover, more cells were observed to detach and floating in the media with the increase of cadmium concentration. (images of cells observed microscope (scale bar: $10 \mathrm{x}$ at $400 \mu \mathrm{m}$ )). 147-148

Figure 5.3: The effect of cadmium chloride $\left(\mathrm{CdCl}_{2}\right)$ on GRP78, HIF $1 \alpha, N F E 2 L 1$ and $N R F 2$ gene expression in human intestinal epithelium cell 6 (HIEC-6) and human colon cancer adenocarcinoma (HCT116) cells. HIEC-6 and HCT116 cells were treated with 0,2.5, 5, and $10 \mu \mathrm{M} \mathrm{CdCl}_{2}$ (24hrs). Cells without treatment were used as controls. Total RNA was isolated from HIEC-6 and HCT116 cells, converted to cDNA and analyzed by qPCR using gene-specific primers for a) GRP78, b) HIF 1 $\alpha$, c) NFE2L1 and d) NRF2 and target genes. The level of target genes of each mRNA was normalized to the GAPDH mRNA. Data are the average from three independent experiments \pm S.E.M. Data analysis 
was done using one-way ANOVA followed by Dunnett's test; ${ }^{*} \mathrm{p}<0.05, * * \mathrm{p}<0.01, * * *$ $\mathrm{p}<0.001$

150-151

Figure 5.4: The effect of cadmium chloride $\left(\mathrm{CdCl}_{2}\right)$ on $M T 1$ gene expression in human intestinal epithelium cell 6 (HIEC-6) and human colon cancer adenocarcinoma (HCT116) cells. HIEC-6 and HCT116 cells were treated with $0,2.5,5$, and $10 \mu \mathrm{M} \mathrm{CdCl}_{2}$ (24 hrs). Cells without treatment were used as controls. Total RNA was isolated from HIEC-6 and HCT116 cells, converted to cDNA, and analyzed by qPCR using gene-specific primers for a MT1 target genes. The level of target genes of each mRNA was normalized to the GAPDH mRNA. Data are the average from three independent experiments \pm S.E.M. Data analysis was done using one-way ANOVA followed by Dunnett's test; *p $<0.05$, ** $\mathrm{p}<0.01, * * * \mathrm{p}<0.001$

$152-153$

Figure 5.5: The effect of cadmium chloride $\left(\mathrm{CdCl}_{2}\right)$ on PORCN, WLS, WNT3A and WNT5A gene expression in a) human intestinal epithelium cell 6 (HIEC-6) cells and b) human colon cancer adenocarcinoma (HCT116) cells. HIEC-6 and HCT116 cells were treated with $0,2.5,5$, and $10 \mu \mathrm{M} \mathrm{CdCl}_{2}(24 \mathrm{hrs})$. Cells without treatment were used as controls. Total RNA was isolated from HCT116 cells, converted to cDNA, and analyzed by qPCR using gene-specific primers for PORCN, WLS, WNT3A, and WNT5A and target genes. The level of target genes of each mRNA was normalized to the GAPDH mRNA. Data are the means from three independent experiments \pm S.E.M. Data analysis was done using one-way ANOVA followed by Dunnett's test; ${ }^{*} \mathrm{p}<0.05, * * \mathrm{p}<0.01, * * * \mathrm{p}<0.001$. 
Figure 5.6: The effect of $\mathrm{CdCl}_{2}$ on $\square$-catenin, PORCN, WLS and WNT3A protein expression in (a-d) human epithelium intestinal cells (HIEC-6) and (e-h) human colorectal cancer tumor (HCT116). Cells were treated with $\mathrm{CdCl}_{2}$ at $0,2.5,5$, and $10 \mu \mathrm{M}$ concentrations. a) The Western blot analysis showed that (a-d) and (e-h) corresponding to $\beta$-catenin, PORCN, WLS and WNT3A protein expression in HIEC-6 and HCT116 cells respectively. Figure (i) and (j) correspond to the densitometric quantification profile of genes in HIEC-6 and HCT116 cells respectively. The level of protein expression of each sample was normalized to $\beta$-tubulin. Data are the mean band densities from three independent experiment \pm S.E.M. Data analysis was done using one-way ANOVA followed by Dunnett's test; * $\mathrm{p}<0.05$, ** $\mathrm{p}<0.01, * * * \mathrm{p}<0.001$.

158-159

Figure 5.7: The effect of $\mathrm{CdCl}_{2}$ on GRP78, HIF1 $\square$, NFE2L1 and NFE2L2 protein expression in (a-d) human epithelium intestinal cells (HIEC-6) and (e-h) human colorectal cancer tumor (HCT116). Cells were treated with $\mathrm{CdCl}_{2}$ at $0,2.5,5$, and $10 \mu \mathrm{M}$ concentrations. Figure (i) and (j) correspond to the densitometric quantification profile expression of genes in HIEC-6 and HCT116 cells respectively. The level of protein expression of each sample was normalized to $\beta$-tubulin. Data are the mean band densities from three independent experiment \pm S.E.M. Data analysis was done using one-way ANOVA followed by Dunnett's test; ${ }^{*} \mathrm{p}<0.05, * * \mathrm{p}<0.01, * * * \mathrm{p}<0.001$.

158-159

Figure 5.8: The effect of $\mathrm{CdCl}_{2}$ on $\mathrm{MT} 1$ protein expression in a) human epithelium intestinal cells (HIEC-6) and b) human colorectal cancer tumor (HCT116). Cells were 
treated with $\mathrm{CdCl}_{2}$ at $0,2.5,5$, and $10 \mu \mathrm{M}$ concentrations. The corresponding densitometric quantification profile represents the measurement of protein expression. The level of protein expression of each sample was normalized to $\beta$-tubulin. Data are the mean band densities from three independent experiment \pm S.E.M. Data analysis was done using oneway ANOVA followed by Dunnett's test; ${ }^{*} \mathrm{p}<0.05,{ }^{* *} \mathrm{p}<0.01,{ }^{* * *} \mathrm{p}<0.001 . \quad \mathbf{1 6 0 - 1 6 1}$ 


\author{
Abbreviations \\ 2BHDA:2-bromo-hexadecanoic acid \\ AA: Antimycin A \\ ABC: ATP-binding cassette \\ ALP: alkaline phosphatase \\ ARE: Antioxidant response element \\ APC: Adenomatous polyposis coli \\ ATF-6: Activating transcription factor 6 \\ Cd: Cadmium \\ $\mathrm{CdCl}_{2}$ : Cadmium chloride \\ CamKII: $\mathrm{Ca}^{2+} /$ calmodulin-dependent protein \\ CIN: Chromosomal instability \\ CK1: Casein kinase 1 \\ CRC: Colorectal cancer or colorectal carcinoma \\ COX: Cyclooxygenases \\ DAG: Diacylglycerol \\ DFO: Deferoxamine \\ DMOG: Dimethyloxallyl glycine \\ DTT: Dithiothreitol \\ DKK-1: Dickkopf related-protein-1 \\ Dvl: Disheveled \\ EMSA: Electrophoretic mobility shift assay \\ RNF: RING finger protein receptor \\ ER: Endoplasmic reticulum \\ ERK: Extracellular signal-regulated kinase \\ FZD: Frizzled protein \\ GPCR: G protein-coupled receptor \\ GRP78 Glucose regulatory protein 78 \\ GSK-3: Glycogen synthase kinase-3 \\ HCT116: Human colon tumor cell \\ $\mathrm{H}_{2}$ DCFDA: 2',7'-dichlorodihydrofluorescein diacetate \\ HEK293T: Human embryonic kidney 293 transformed \\ HIEC-6: Human Intestinal epithelial cell 6 \\ HIF1 $\alpha$ : Hypoxia inducible factor 1 alpha \\ HRE: Hypoxia response element \\ $\mathrm{IP}_{3}$ : Inositol 1,4,5-trisphosphate \\ IRE1: Inositol-requiring protein 1 \\ JNK: c-Jun N-terminal kinases \\ LEF: Lymphoid enhancer binding factor \\ LGR: Leucine-rich repeat-containing $\mathrm{G}$ protein coupled receptor \\ LOX: Lipoxygenases \\ LPR: Low density lipoprotein receptor related protein \\ MG132: Proteasome inhibitor \\ MBOAT: Membrane bound $\mathrm{O}$ acyltransferase
}


MSI: Microsatellite instability

MPO: Myeloperoxidase

MSC: Mesenchymal stem/stromal cell

MSI: Microsatellite instability

MT1: Metallothionein

MTT: 3-(4,5-dimethylthiazol-2-yl)-2,5-diphenyl-2H-tetrazolium bromide

NADPH: nicotine adenine dinucleotide phosphoric acid

NFE2L1 and and 2: Nuclear factor, erythroid 2 like 1 and 2

NF- $\kappa \beta$ : Nuclear factor kappa-light-chain-enhancer of activated $\beta$

O-PAM: O-palmitoyleation

OST: Oligosaccharyltransferase

PCP: Planer cell polarity

PERK: PKR-like endoplasmic reticulum kinase

PKA: Protein kinase A

PKC: Protein kinase $\mathrm{C}$

PORCN: Porcupine

PPAR $\delta$ : Peroxisome proliferator-activated receptor, delta isoform

PTM: Post-translational Modification

ROS: Reactive oxygen species

SCD 1: Stearoyl-CoA desaturase 1

TAM: Tumor associated microphages

TCF T: T-cell factor-specific transcription factor

TMEM 132A: Transmembrane protein 132A

Thap: Thapsigargin

Tuni: Tunicamycin

TMD: Transmembrane domain

UPR: Unfolded protein response

Vps: Vacuolar protein sorting

WHO: World Health Organization

WIF: WNT inhibitory protein

Wg: Wingless

WNT: Wingless/int-1

XO: Xanthine oxidase 
The true splendor of science is not so much that it names and classifies, records and predicts, but that it observes and desires to know the facts, whatever they may turn out to be.

\author{
By Alan Watts
}

Research is to see what everybody else has seen, and think what nobody else has thought.

by Albert Szent-Gyorgyi 


\section{Chapter 1: Introduction}

\subsection{Colon Cancer}

Colorectal cancer (CRC) is classified as the third most common malignancy worldwide as it accounts for approximately $10 \%$ of all cancer incidence worldwide. Moreover, it is the fourth most prevalent cause of death among other types of cancers (Stone et al., 2014). Fig. 1 is showing the incidence rate across the globe. There are few main molecular contributors to CRC including the chromosomal instability (CIN) and microsatellite instability (MSI). These instabilities lead to DNA damage induced by environmental carcinogens or mutations arising from replication errors (Stone et al., 2014). Furthermore, there are several risks associated with CRC development such as environmental toxins, smoking, physical inactivity, obesity, infections, and diet (Carini et al., 2017).

Cancer is controlled by genetic and epigenetic alterations that allow cells to overproliferate and escape mechanisms that normally control their survival and migration. Many of these changes are directed to signaling pathways that control cell growth and division, cell death, cell fate, and cell motility, and can be placed in the context of distortions of wider signaling networks that fuel cancer progression, such as changes in the tumor microenvironment, angiogenesis, and inflammation (Schatoff et al., 2017). Mutations that convert cellular proto-oncogenes to oncogenes can cause hyperactivation or deregulation of these signaling pathways, one of the examples is WNT signaling pathway (Zhan et al., 2017). WNT name is generated from the Drosophila segment polarity gene wingless and the name of the vertebrate homolog, integrated or int-1 (Woodraz and Nusse, 1998). WNT signaling cascades are cutting-edge topics in the field of translational 
oncology and medicinal chemistry; since WNT signaling cascades are the major driver of various types of human cancers including colon cancer. 


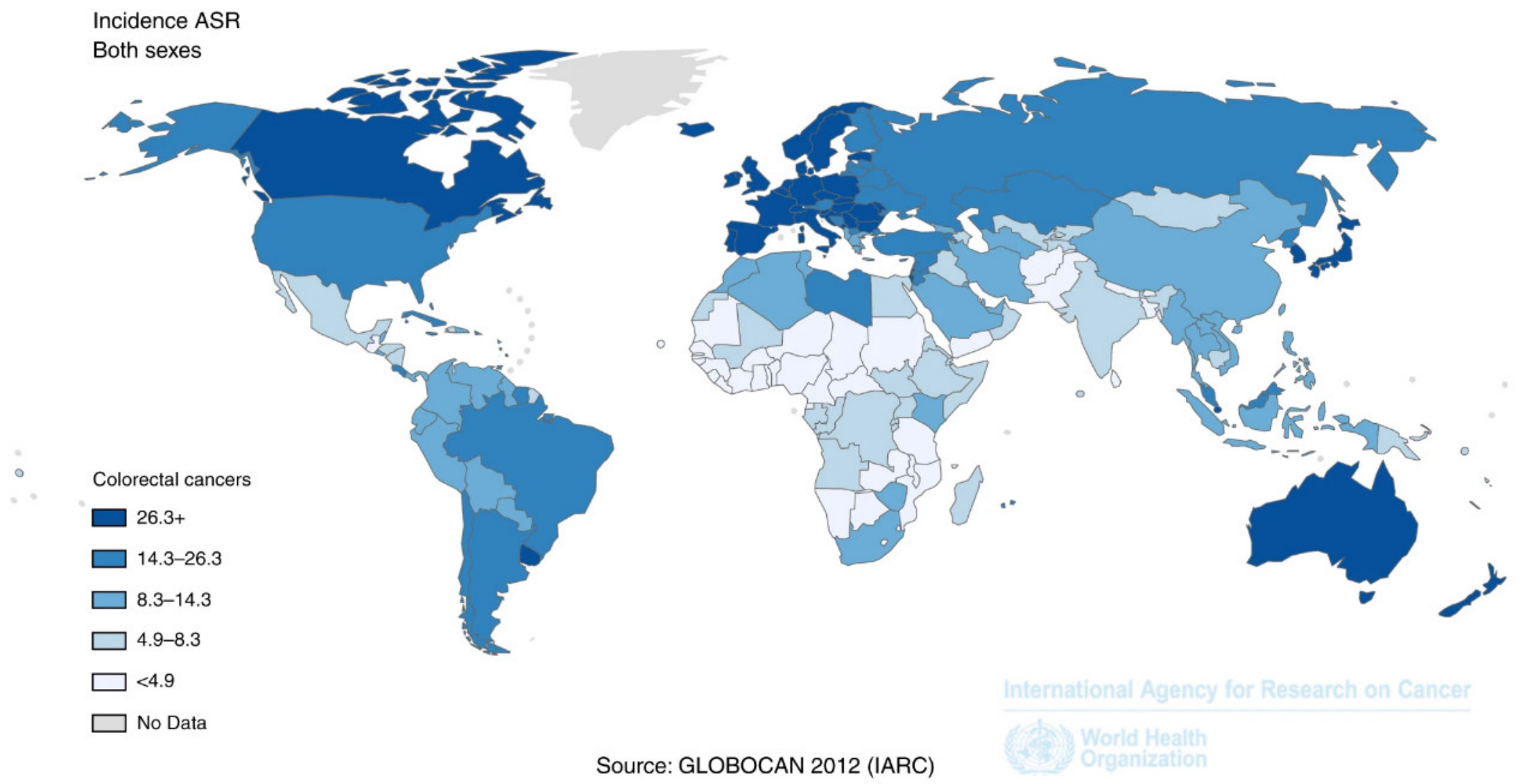

Figure 1.1: Estimated age-standardized incidence rate (standard: world population) of colorectal cancer in 2012 (source: Globocan 2012, International Agency for Research on Cancer). 


\subsection{The emerging roles of WNT in human colon cancer}

CRC is the third leading cause of cancer-related death in the world and accounts for almost 600,000 deaths worldwide (Fig. 1.1, Globocan, 2012), annually (Jemal and Bray, 2011). The aberrant activation of WNT signaling on the development of colorectal cancer is well-documented (Polakis, 2007). It is well-known that WNT overexpression could lead to malignant transformation. Almost all CRCs demonstrate hyperactivation of the extracellular WNT production, which in many cases is believed to be the initiating and driving event (Schatoff, et al., 2017). The WNT pathway continues to evolve as a central mechanism in cancer biology (Nusse, 2006). Identified components and processes include the WNT secretory machinery, WNT co-receptors, components of the $\beta$-catenin destruction complex and nuclear co-factors that have led to the activation of $\mathrm{WNT} / \beta$ catenin pathway and their impact and development of cancer (Clevers, 2006). One of the key factors for causing colon cancer is the loss of APC, which is the main driver $\beta$-catenin destruction in theWNT signaling and its important role was further highlighted by several recent studies (Mantona et al., 2015; Drost et al., 2015). Furthermore, studies of human CRC samples and tumors from mouse models revealed that different mutations of APC result in distinct levels of canonical WNT pathway activity (Flangan et al., 2019; Figure 1.1) and are associated with characteristic tumor locations within the large intestine (Rubinfeld et al., 1997). Using a mouse model with reversible knockdown of APC via shRNA, it was demonstrated that adenomas could regress to normal tissue if APC (a tuomor suppressor) function is restored, underlining the importance of continuous WNT signaling for tumor maintenance (Morin et al., 1996). Moreover, it was also shown that in spite of truncated APC, WNT pathway activity can still be modulated by interference with 
WNT secretion (Figure 1.2) (Morin et al., 1996). Due of the complexity of WNT signaling cascades and genetic alterations in non-enzymatic signaling components, it has become a major interest in our lab to investigate the secretory pathway under different stressors.

In our current study used (HCT116) human colorectal cancer cell line is commonly used for the study of WNT signaling pathways. It possesses a mutation in $\beta$-catenin that caused it to be overexpressed in this cell line (Sekine et al., 2002). Target disruption of the mutant $\beta$-catenin gene in colon cancer cell line HCT116 preservation of its malignant phenotype and thus, it is highly sensitive to WNT signaling (Proto et al., 2017). HIEC-6 cells are a normal human small intestinal epithelial cell line which is used in the investigation of the regulation of normal intestinal cell proliferation, survival, stemness, as well as studies of the molecular mechanisms leading to lineage-specific differentiation. HIEC-6 cells are also used for the study of the effects of hormones, growth factors, extracellular matrix molecules, drug metabolism, and carcinogenic factors on small intestinal functions (Lanerholc et al., 2011). We utilized these two cell lines in order to compare a normal and cancerous intestinal cell line for the responses to stressors normally found in the tumor microenvironment. 


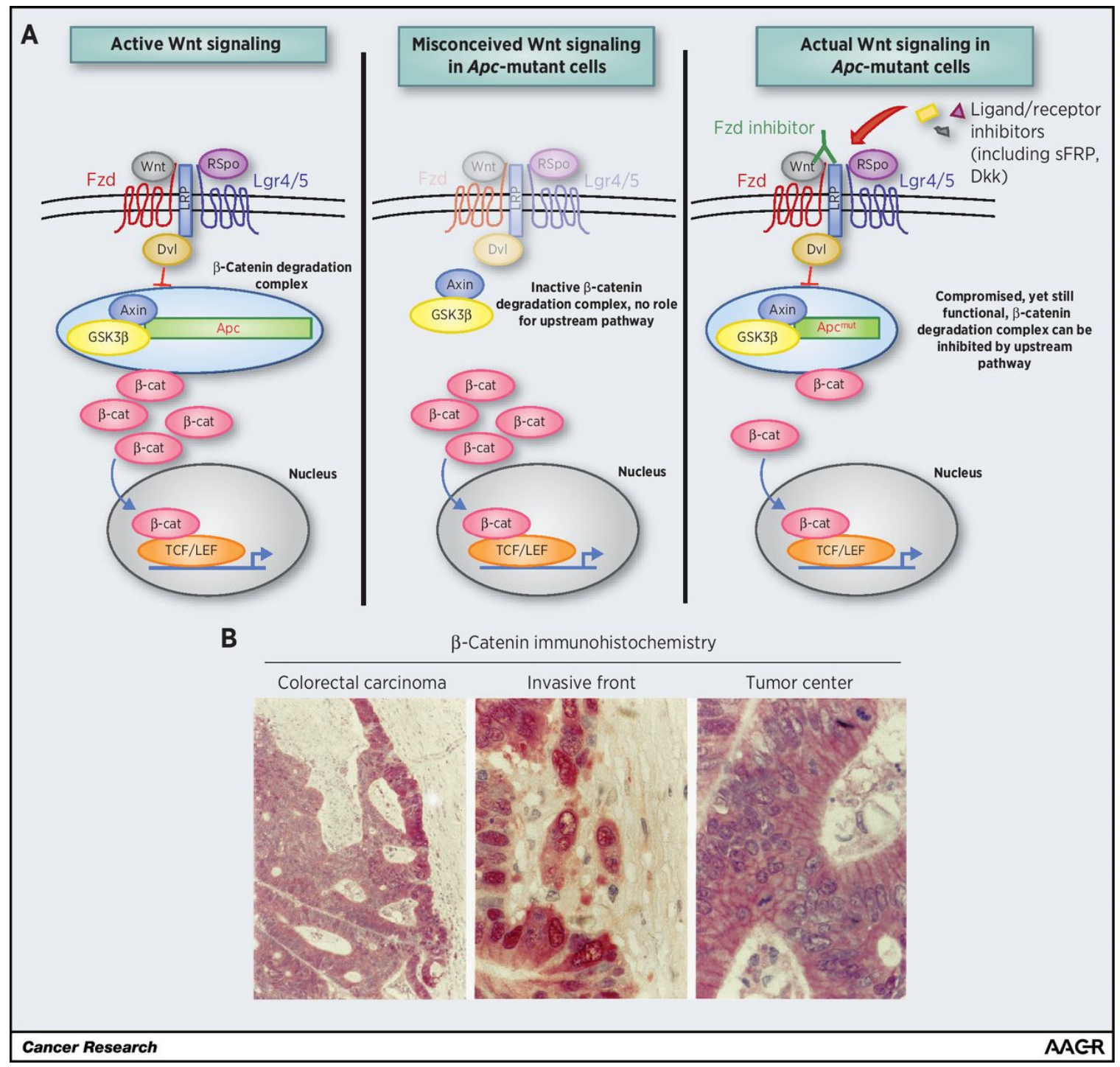

Figure 1.2: WNT signalling affects the function and structure of the colon tissue. WNT signalling with mutant APC is the main cause of colon cancer. The image on the left illustrates WNT signaling in its active form, with the continuous degradation of $\beta$-catenin. The middle image shows the condition when APC is deleted, which affects the activity of WNT signalling. The image on the right shows' inhibitors of WNT signalling in Apcmutant cells. $\mathrm{B}$, immunohistochemistry for $\beta$-catenin in a human colorectal carcinoma 
showing increased nuclear localization as a surrogate marker of active WNT in the invasive front compared with the tumor center (Flanagan et al., 2019). 


\subsection{WNT signalling pathway}

Cell communication is vital throughout the development of multicellular organisms and during adult homeostasis. One way in which communication is achieved is through the secretion of signaling molecules that are received by neighboring responding cells. There is accumulated evidence that has demonstrated that long term stability of tissue function and structures and organization of tissues and organs rely on the precise regulation of stem cell regeneration, WNT proteins function via cell surface receptors to stimulate an increase in intracellular $\mathrm{Ca}^{2+}$ and the subsequent activation of protein kinase $\mathrm{C}$ (PKC) (Miller et al., 1999; Umar, 2010). The WNT signalling pathway involved in cell-cell communication and activation of critical transcription factor cyclin D during mitotic division which allows for cell proliferation and differentiation (Nusse et al., 2008 and Nusse, 2008). WNT ligand initiate intracellular signalling by binding to cell surface-expressed WNT receptors and coreceptors, low density lipoproteins-related proteins, as well as receptor tyrpsine kinase (Ljungberg et al., 2019).

In 1970, genetic screens were done on Drosophila which showed abnormal sets of genes related to the development of the embryo (Nüsslein-volhard and Wieschaus, 1980). The abnormalities ranged from abnormal segment numbers to polarity changes, and mutations which affected segment number and polarity in Drosophila (Nüssleinvolhard and Wieschaus, 1980). A group of unknown genes shared a mutation in their phenotype and were called Wingless $(\mathrm{Wg}$ ) because the mutant gene was found to be responsible for the loss of wing tissue (Hamada et al., 1999). Segmentation of wings were tracked down using gene mapping, which illustrated that the gene responsible for the polarity was close to the $\mathrm{Wg}$ gene (Baker, 1988). Throughout evolution, the amino acid 
sequence of WNT1 remained highly conserved amongst species including Eukaryota; Metazoa; Chordata; Craniata; Vertebrata; Euteleostomi; Mammalia; Eutheria; Euarchontoglires; Primates; Haplorrhini; Catarrhini; Hominidae; Homo, which helped in understanding the evolutionary role of WNT in organisms. This fascinating pathway has profound global effects on cellular function, and defects in this pathway result in disease states (Polakis, 2000).

WNT signalling has and will be the docuss of extensive research because of its key role in many aspects of stem cell especially cancer. Additionally, due to its impact on human health there is a niche of understanding pathological association of dyregulation of WNT signalling (Nusse and Varmus, 2012). This work is inspired by the gap of understanding how WNT and WNT components reponses to stresses during secretion. Furthermore, we are interested to understand how an evolutionary conserved signalling dyregulations gives rise to disease and what currect transcription factors are implicated in this process are.

\subsection{Functions of WNT signalling network}

WNT proteins are a family of 19 secreted glycoproteins which are responsible for cell survival, proliferation, migration and polarity, cell fate specification, body axis patterning and self-renewal in stem cells (Table 1) (Clever, 2001). The WNT proteins contribute to the stabilization of proteins other than $\beta$-catenin, specifically at the $\mathrm{G} 2 / \mathrm{M}$ transition during mitosis. Canonical WNT signalling plays a key mitogenic role in promoting G1 progression by inhibiting GSK3, which directly regulates $\beta$-catenin 
dependent c-myc transcription, cell-cycle effectors and growth regulator (Niehrs and Acebron, 2012).

WNT proteins are involved in a variety of functions throughout the human body including functions in tissue such as brain, liver, breast, reproductive system, gastrointestinal tract, and bone (Fig. 1.3). WNT signalling plays a critical role in the mammalian intestinal epithelium where it regulates stem cell behavior, proliferation, cellular differentiation, apoptosis, and migration (Nusse, 2006). In addition, WNT signaling is essential for normal breast stem cell function and mammary gland development during embryogenesis, postnatal development and pregnancy (van Amerongen et al., 2012) with adult mammary glands containing WNT-responsive stem cell populations (Zeng and Nusse et al., 2010). Furthermore, WNT signaling is a key regulator in adult osteogenic differentiation of mesenchymal stem cells (Houschyar et al., 2019). The WNT signaling pathway is one such signaling cascade that enables hepatic homeostasis and contributes to unique hepatic attributes such as metabolic zonation and regeneration (Russell and Monga, 2018).

In the non-canonical pathway, WNT5A is involved in response to infection which has been also observed to be expressed by tumor-associated macrophages (TAM) (Pukrop et al., 2006). Other studies have shown that components of the WNT signaling pathway impact reproductive functions including embryonic development of the sex organs, and regulation of follicle maturation controlling steroidogenesis in the postnatal ovary (Hernandez, 2015). 


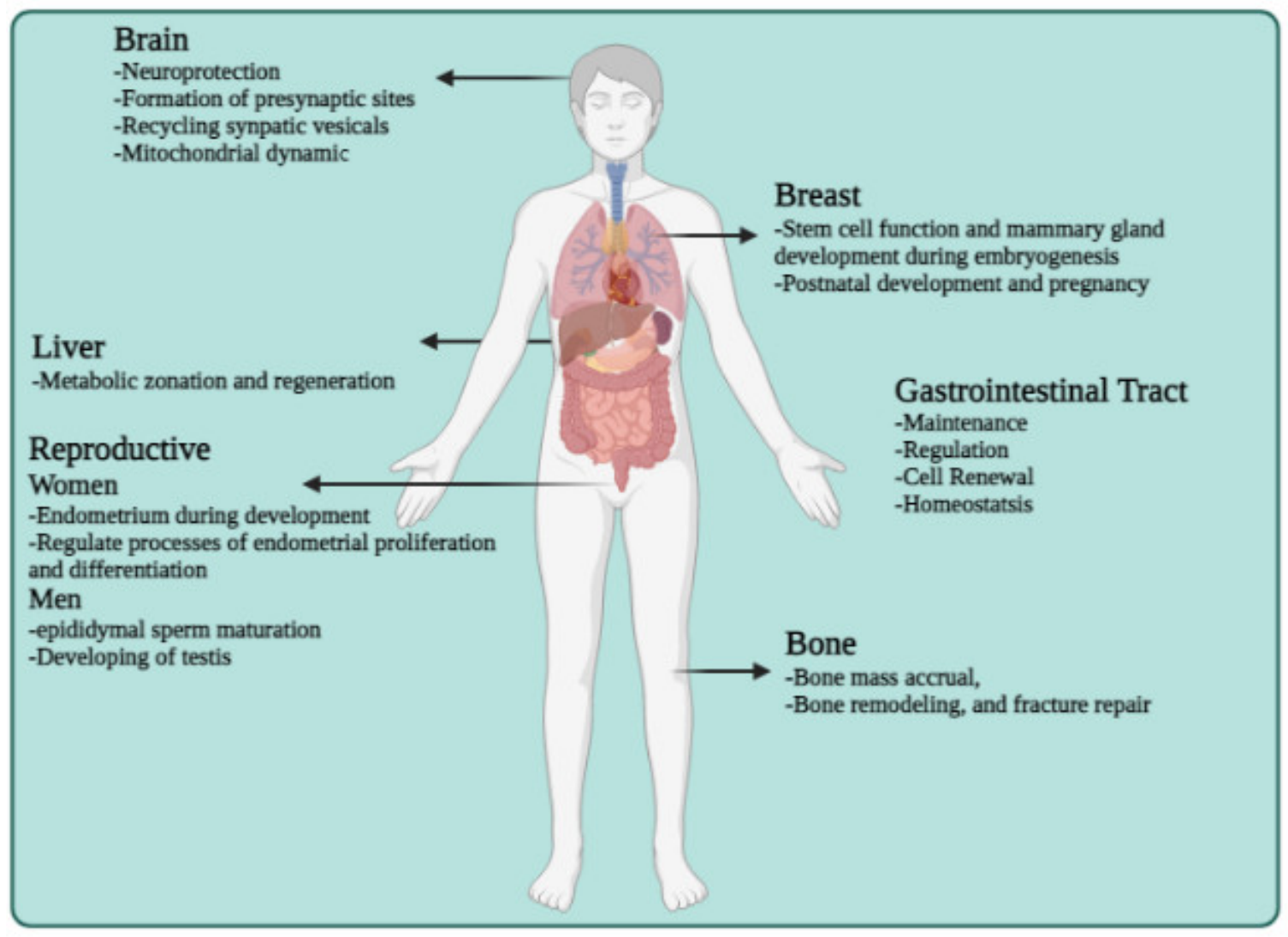

Figure 1.3: Illustrating the role and function of WNT signaling in different organs and tissues in the human body. The role of WNT can be seen in every part of the human body, and specifically the vital organs (brain, liver, breast, reproductive system, gastrointestinal tract, and bone). (image created by R. Mohamed using BioRender). 


\subsection{The WNT ligands}

Ligand is a molecule that binds to another molecule, used especially to refer to a small molecule that binds specifically to a larger molecule; also known to form a coordinate covalent bond with the central metal atom of a coordination complex (Dorland, 1988). WNT ligands bind to various receptors present on receiving cells, WNTs initiate intracellular signaling cascades resulting in changes in gene transcription (Wu and Nusse, 2002). WNT genes encode for a large number of conserved proteins which are characterized by the WNT motif (C-K-C-H-G-(LIVMT)-S-G-X-C); X can be any amino acid; and including a nearly invariant positioning of 22 cysteines residues with highly charged amino acids residues; the majority of which are marked for potential glycosylation sites (Reichsman et al., 1996).

The WNT family encodes secreted glycoproteins of molecular mass less than 35 $\mathrm{kDa}$ (Clevers, 2006). WNT proteins are known to be hydrophobic and imparted by lipidation. The hydrophobicity of WNTs where it makes multiple contacts to completely traverse the cysteine rich domain surface, as well as it ensure the insertion of WNT to the hydrophobic grooves of Frizzled binding sites (Willert and Nusse, 2008). WNT comprises 350-400 amino acids in length (Logan and Nusse, 2004). The WNT proteins carry several highly charged amino acids and have a predicted isoelectric point of nearly 9 (Willert and Nusse, 2012). WNT3A represents the first well-characterized WNT protein (Willert et al., 2003). Furthermore, the expression of WNT3A was associated in various stages of colorectal cancer cells from primary tumor to metastatic (Lee et al., 2014). WNT signaling is divided into canonical and non-canonical pathways. WNT family comprises 19 human proteins (Table 1), including WNT1, WNT2A, (WNT2A/13), WNT3, WNT3A, WNT4A, 
WNT5A, WNT5B, WNT6A, WNT7A, WNT8A, WNT8B, WNT9A, WNT10A, WNT10B, WNT11A, WNT11, and WNT16 (Bodine, 2008). The WNTs, and their associated proteins, control cells through three downstream signalling pathways that include 1) the canonical $\beta$-catenin-dependent pathway, 2) the noncanonical planar cell polarity (PCP) pathway and 3) the noncanonical WNT/calcium pathway (Komiya and Habas, 2008). The critical role of $\beta$-catenin is a key downstream component of the canonical WNT signaling pathway and initiates important function in epithelial cells in gastric cancer, specifically cell-cell adhesion and coordination and gene transcription (Bhattacharyya et al., 2014). 
Table 1: List of mammalians WNTS and their functions. The WNT proteins contain 19 different family members of WNT and are essential secretory factors involved in WNT pathways (canonical and non-canonical).

\begin{tabular}{|c|c|c|}
\hline WNT Type & WNT Family & Function \\
\hline \multirow{15}{*}{$\begin{array}{l}\text { Canonical } \\
\text { Pathway }\end{array}$} & WNT1 & Induction of the mesencephalon and cerebellum (Keupp et al., 2013) \\
\hline & WNT2A & $\begin{array}{l}\text { Functions in the canonical WNT signaling pathway that results in activation } \\
\text { of transcription factors of the TCF/LEF family (Katoh, 2005) }\end{array}$ \\
\hline & WNT2A/13 & Spatial and temporal arragements (Katoh, 2005) \\
\hline & WNT3 & $\begin{array}{l}\text { Embryonic development of early stage of human limb formation, craniofacial } \\
\text { and urogenital development (Niemann, et al., 2004) }\end{array}$ \\
\hline & WNT3A & $\begin{array}{l}\text { Non redundantly controls function of fetal liver hematopoietic stem cells and } \\
\text { Promotes osteogenic differentiation (Yue et al., 2008) }\end{array}$ \\
\hline & WNT5B & Coordinates chondrocyte proliferation and differentiation (Uhlén, et al., 2015) \\
\hline & WNT6A & $\begin{array}{l}\text { Formation and development of embryonic structures (fetal heart, and ventral } \\
\text { body wall) (Yang et al., 2003) }\end{array}$ \\
\hline & WNT7A & $\begin{array}{l}\text { Development of anterior-posterior axis of female reproductive tract (Uhlén et } \\
\text { al., 2015) }\end{array}$ \\
\hline & WNT8A & Development of patterning in early embryos (Gao et al., 2013) \\
\hline & WNT8B & $\begin{array}{l}\text { Suppresses formation of early eye and retinal progenitor cells in vertebrates } \\
\text { (Yang et al., 2003) }\end{array}$ \\
\hline & WNT9A & Development of cell proliferation in AV canal (Weissenböck et al., 2019) \\
\hline & WNT10A & Development of ectodermal appendages (Nawaz et al., 2009) \\
\hline & WNT10B & $\begin{array}{l}\text { Role in signalling networks to control stemness, pluripotency, and cell fate } \\
\text { (Wend et al., 2012) }\end{array}$ \\
\hline & WNT11A & $\begin{array}{l}\text { Plays a role in regulating heart-chamber proliferation during prenatal } \\
\text { maturation } \\
\text { (Touma et al., 2017) }\end{array}$ \\
\hline & WNT16A & $\begin{array}{l}\text { Expression of WNT16B: increases cell proliferation and prolonges } \\
\text { clonogenicity in primary keratinocytes (Teh et al., 2007) }\end{array}$ \\
\hline \multirow{3}{*}{$\begin{array}{l}\text { Non-canonical } \\
\text { Pathway }\end{array}$} & WNT4A & $\begin{array}{l}\text { Sex determination, kidney formation, adrenal glands, gonads, and responsible } \\
\text { for breast development (Fiontana et al., 2017) }\end{array}$ \\
\hline & WNT5A & $\begin{array}{l}\text { Development of limbs, tails, and facial structures } \\
\text { (Yang et al., 2003) }\end{array}$ \\
\hline & WNT11 A & Development of ectodermal appendages (Xu et al., 2017) \\
\hline \multirow{2}{*}{$\begin{array}{c}\text { WNT } \\
\text { Secretory } \\
\text { Components }\end{array}$} & PORCN & Transport, secrete WNT proteins and modify WNT (PTM)(Nusse, 2006) \\
\hline & WLS & Transport WNT and modify WNT (PTM) (Nusse, 2006) \\
\hline
\end{tabular}




\subsection{WNT signaling pathways}

\subsubsection{Canonical WNT/ $\beta$-catenin-dependent}

A key mediator of WNT signalling, the armadillo protein $\beta$-cadherin-associated protein ( $\beta$-catenin), which contributes to stabilize cell-cell contacts, is tightly controlled by proteins that regulate nuclear stability (Metacalfe and Bienze, 2011; Mosimann et al., 2009). $\beta$-catenin consists of highly specialized proteins that are grouped into three subcategories; 1) P120, 2) $\beta$-catenin and plakoglobin and 3) a distant alpha sub-family (Ozawa et al., 1989; Wieschaus et al., 1984). The function of $\beta$-catenin is not well-defined; however, its involvement in induction of the signal to the nucleus and it triggers transcription of Wnt-specific genes responsible for the control of cell fate decisions in many cells and tissues, has been well documented (He et al., 1997). The WNT signalling pathway regulates several aspects of cell fate during embryonic development, including migration, cell polarity, neural patterning, and organogenesis (Nusse et al., 2009; Komiya and Habas, 2008; He et al., 1989; Korinek et al., 1997; Morin et al., 1997; Morin et al., 1996; Nusse et al., 2008). In the canonical WNT cascade, $\beta$-catenin is the key effector responsible for sending signals to the nucleus, as it triggers transcription of WNT-specific genes responsible for the control of cell fate (Fig. 4) (Tetsu and McCormick, 1999The downstream WNT pathway begins with the modified WNT protein binding to a receptor complex, which contains a) frizzled (Fz), a family of G protein-coupled and low-density related lipoproteins 5 or 6 (LRP5/6) (Logan and Nusse, 2004; and Herz and Strickland, 2001), b) leucine-rich repeat-containing G-protein coupled receptors (LGR) (Huang and Klein, 2004), and c) an evolutionarily conserved E3 ligase RING finger protein receptor (RNF) (Sh 
ae et al., 2000). However, WNT specifically binds to the N-terminus of the cysteine rich Fz receptor (FzR), which transduces the signal to dishevelled (Dsh/Dvl), found in the cytoplasm of the cell (Darom et al., 2010). The Dsh/Dvl phosphoprotein interacts with the FzR to initiate one of three cascades; the canonical WNT pathway (Angers and Moon, 2009), the non-canonical planar cell polarity pathway (Gao, 2012), and the non-canonical WNT/calcium pathway (Kühl et al., 2000). Nevertheless, it was observed that the extracellular WNT signal is responsible for the induction of various intracellular signal transduction cascades in WNT downstream pathways (Komiya and Habas, 2008). FzR are involved in only two of these pathways; the canonical WNT pathway and the non-canonical polarity/PCP signaling (Strutt, 2003). $\beta$-catenin is phosphorylated in the cytoplasm by the activity of a multiprotein destruction complex, marking $\beta$-catenin for degradation (Clevers, 2006). This consists of the scaffold proteins axin and Adenoma Polyposis Coli (APC), and of the kinases/phosphatases responsible for phosphorylating $\beta$-catenin; glycogen synthase kinase $3 \beta(G S K 3 \beta)$, casein kinase 1 (CK1) and protein phosphatase 2A (PP2A) (Kimelman and $\mathrm{Xu}, 2006$ ). The binding of WNT ligands to the transmembrane FzR and LRP5/6 coreceptors leads to inhibition of the $\beta$-catenin destruction complex (Clevers, 2006). Ligandactivated FzRs recruit cytoplasmic protein Dsh/Dvl to the receptor complex through immediate binding. Dsh/Dvl then multimerizes and induces formation of the so-called LRP-associated WNT (Bilić, et al., 2007). Dsh/Dvl activates the rate-limiting component axin, which destabilizes the $\beta$-catenin destruction complex (Schwarz-Romond et al., 2007). Dsh/Dvl initiates phosphorylation of LRP5/6 by CK1, which leads to an inhibition of GSK3 kinase activity (Zeng et al., 2008). Unphosphorylated $\beta$-catenin escapes degradation, accumulates in the cytoplasm, and translocates to the nucleus. Nuclear $\beta$-catenin associates 
with DNA-binding transcription factors of the $\mathrm{T}$ cell factor/lymphoid enhancer factor family (TCF/Lef) family (Axelrod, 2001). In the absence of WNT signals, TCF/Lef factors act as transcriptional repressors. The binding of $\beta$-catenin converts TCF/Lef proteins into two separate $\mathrm{TCF} / \beta$-catenin transcriptional activators, converting the WNT signal into the transcription of specific target genes (Novellasdemunt et al., 2015). There are currently 19 different types of WNT family members known. The first int1 protein, now known as WNT1 protein, was isolated in 1982 by Nusse and Varmus (Nusse and Varmus, 2012). Table 1 shows various WNT family members.

\subsubsection{Non-canonical WNT pathway}

The non-canonical WNT pathways broadly encompass a range of WNT activated pathways that do not involve $\beta$-catenin (Katoh et al., 1996 and Nishimura et al., 2012). Non-canonical WNT signaling cascades are involved in maintenance of stem cells, directional cell movement, or inhibition of the canonical WNT signaling cascade (Katoh

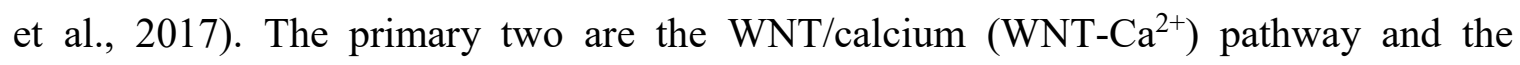
JNK/planer cell polarity (PCP) pathway (Katoh et al., 1996; Nishimura et al., 2012). The WNT/calcium and PCP pathways both utilize Fzd as the cell surface receptor and Dvl within the cell, but they do not involve the LRP coreceptors and result in intracellular cascades that differ to that of the WNT/ $\beta$-catenin pathway (Katoh et al., 2017). Though it is the WNT/ $\beta$-catenin pathway in which this research will focus, it must be noted that there is potential competition between non-canonical and WNT/ $\beta$-catenin pathways for WNT binding to Fzd, and for the intracellular action of Dvl. Furthermore, a number of studies 
have shown suppression of WNT/ $\beta$-catenin signalling by non-canonical WNT signaling (MacDonal et al., 2009) (Fig. 1.4).

\subsubsection{WNT/calcium pathway}

The WNT/calcium pathway is characterized by a G-protein dependent release of calcium resulting in an increase of intracellular calcium concentration which leads to the activation of calcium sensitive enzymes including the protein kinases c (PKC), CamKII, or the protein phosphatase calcineurin (Rao and Kuhl, 2010). Elevated $\mathrm{Ca}^{2+}$ can activate the phosphatase calcineurin, which initiate to dephosphorylation of the nuclear factor of activated T-cells (NFAT) leading to accumulation in the nucleus (Fig. 1.3). The $\mathrm{Ca}^{2+}$ mediate pathway is essential in the roles of dorsal/ ventral patterning, gastrulation, and cardiac development (Capdevila and Belmonte, 2001; Mosimann et al., 2009).

\subsubsection{WNT/PCP pathway}

The WNT/PCP pathway is characterized by the activation of rho-GTPases that regulate c- June N-terminal kinase (JNK). Together with rho-GTPases regulate the actin cytoskeleton (Rao and Kuhl, 2010; Kestler and Kuhl, 2008). The WNT/PCP ligands, such as WNT5A, WNT7A, and WNT11A, bind to the 7 transmembrane Fzd receptor to recruit Dvl to the pathway (Fig. 1.4). 


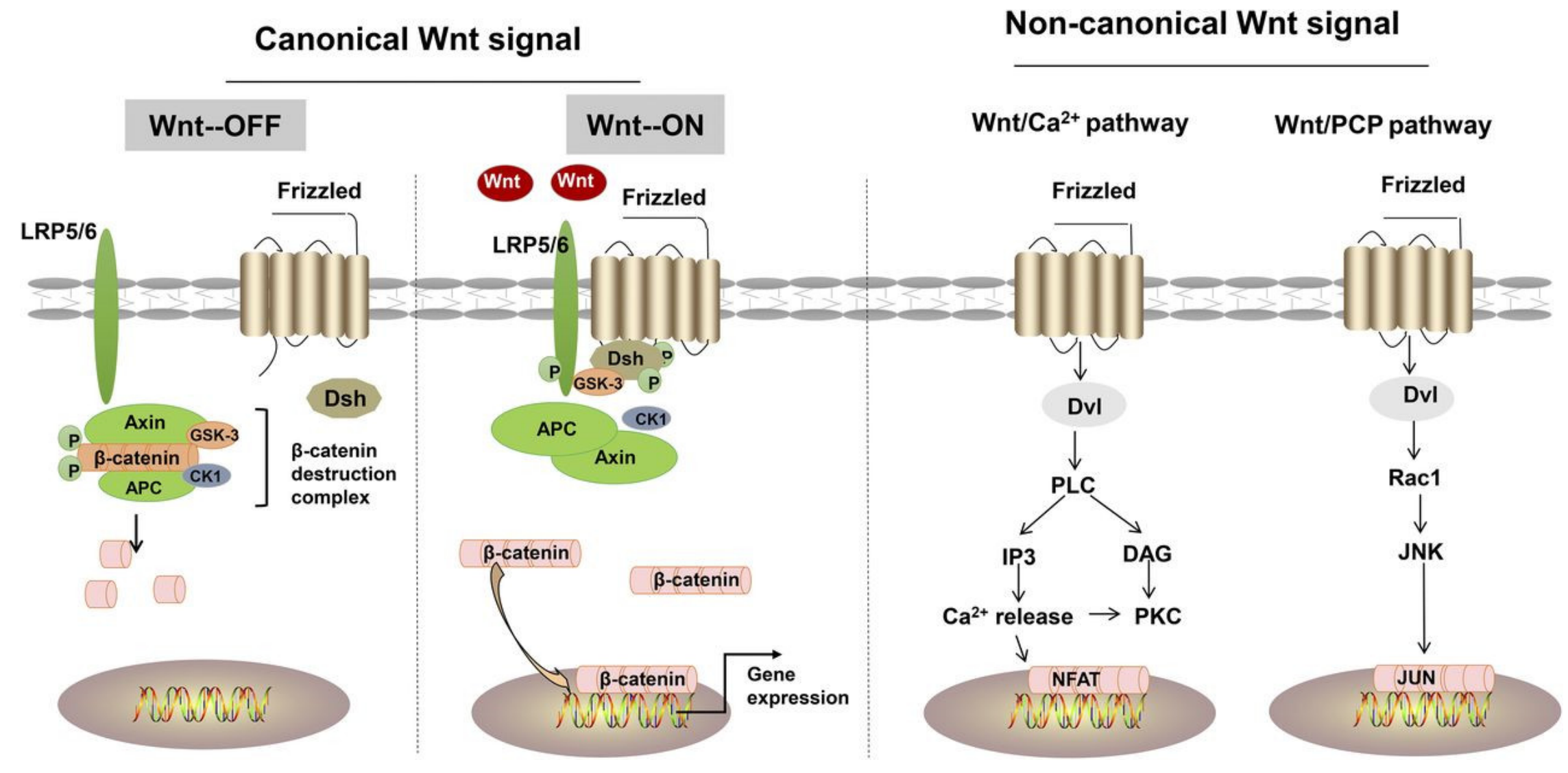


Figure 1.4: Schematic overview of WNT signaling pathways (a) WNT-OFF: in the canonical WNT pathway, the absence of WNT signaling leads to proteasome degradation of $\beta$-catenin that mediate destruction complex including several proteins (GSK-3 $\beta$ and CK1 $\alpha$ kinases, result in $\beta$-catenin phosphorylation). (b) WNT-ON: the activation of WNT signaling start of the WNT ligand binding to its Fz receptor to LRP5/6 co-receptor to the initiate DVL together with the destruction complex to the cell membrane. However, the destruction box that includes GSK-3 $\beta$ and CK1 $\alpha$ mediated phosphorylation of $\beta$-catenin, is inhibited and the stabilized $\beta$-catenin is translocated to the nucleus where it serves as co-activator for Tcf/LEF transcription factors. (c) Non-canonical pathway WNT/Ca ${ }^{2+}$, the interaction of WNT ligands to a specific receptor activates the PLC, initiate intracellular $\mathrm{Ca}^{2+}$ release result in activation of PKC and CaMKII that regulate the activation of NF-AT transcription factor and gene expression. (d) In the non-canonical WNT/PCP signaling pathway, the binding of WNT ligand to the receptor triggers a signalling cascade that involves small Rho-family GTPases and downstream effectors such as JNK and ROCK, which in turn modulate cytoskeleton organization and gene expression (Qu et al., 2019). 


\subsection{The WNT secretory route and modification}

WNT ligand secretion is a strictly regulated, complex process. This tight regulation is in part a consequence of the post-translational modifications carried by WNTs (Harterink and Korswagen, 2012). Multiple studies have shown that WNT proteins are glycosylated and palmitoleated in the endoplasmic reticulum (ER) (Fig. 1.5) (Willert et al., 2003). The palmitoleation process is a lipid modification of cysteine residues, which initiates targeting of the WNT proteins to the plasma membrane. This is an essential step for WNT protein secretion and activity (Gao and Hannoush, 2014). While the WNT proteins are being translated in the ER, they are palmitoleated by the ER bound o-acyltransferase protein called Porcupine (PORCN) (Van Den Heuvel et a., 1993). Once the palmitoyleation of WNT is completed, it undergoes glycosylation by the GPR177 (WNTless or WLS) proteins, this is the action of attaching a carbohydrate to a molecule of interest. WLS as secretion mediator acts as an essential regulator controlling protein glycosylation (Wang et al., 2021). Furthermore, WLS as mediator allows the protein to mediate endosome trafficking and further to release into the extracellular space (Kurayoshi et al., 2007).

In the presence of canonical WNT ligands, $\beta$-catenin phosphorylation and degradation is inhibited. Gene expression downstream of canonical WNT signaling is regulated by controlling the amount of the transcriptional co-activator $\beta$-catenin (Bartscherer et al., 2006). (adapted and modified from Gonzalez-Fernandez et al., 2016a). 


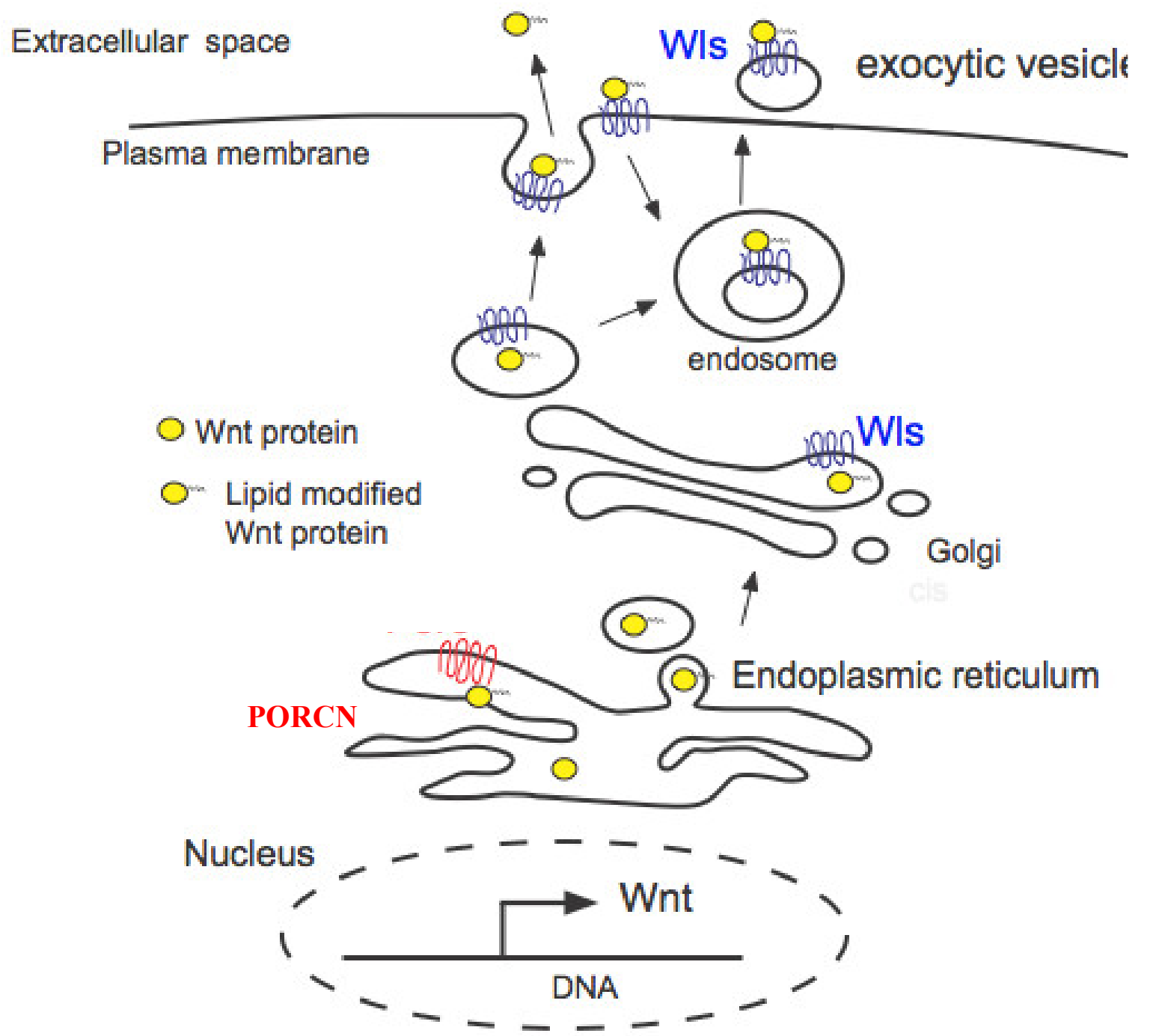

Figure 1.5: Showing the upstream pathway of WNT signalling, highlighting all the essential components of WNT secretions. Upon translation, WNT proteins undergo a series of modifications as they transit through the secretory pathway and associate with several proteins, including Porcupine (here shown as Porc) in the endoplasmic reticulum. All WNT proteins harbor a covalent lipid modification: a palmitoleic acid, attached by the palmitoyltransferase Porc. The transmembrane protein WNTless (WLS) binds exclusively to lipidated WNT proteins and transfers them to the plasma membrane for secretion (Gonzalez-Fernandez et al., 2016a). 


\subsubsection{Porcupine (PORCN)}

PORCN is a ubiquitously expressed upstream regulator of WNT palmitoleation that is secreted and localized to the ER (Tanaka et al., 2002; Tabaczar et al., 2017). PORCN is a multipass transmembrane proteins are spanning the membrane more than once and belonging to the membrane-bound O-acyltransferases (MBOAT) superfamily (Fig. 1.6) (Tanaka et al., 2017; Hofmann, 2000). Mutations in PORCN are associated with focal dermal hypoplasia (FDH; a heritable disorder that causes abnormal development of hair, teeth, nails and glands), whereas gene deletion and/or knockout cause embryonic lethality in mice (Thorpe et al., 1997; Grzeschik et al., 2000). PORCN encodes a protein of 525 amino acids with an estimated molecular weight of $51 \mathrm{kDa}$ and is hydrophobic (Grzeschik et al., 2000). It has 11 predicted transmembrane domains (TMD) that are linked to C77 and S209 (Rios-Esteves, et al., 2014; Tabaczar et al., 2017). Only three members, PORCN, Hedgehog acyltransferase (Hhat), and ghrelin O-acyltransferase (GOAT), have been shown to have protein substrates (Grzeschik et al., 2000). PORCN function is not well defined, it is known to participate in wing patterning of the discs in Drosophila (Hofmann, 2000). The histidine in the acyl transferase active site of PORCN is required for WNT modification which indicates that PORCN is the enzyme that directly modifies WNT proteins (Hartmann et al., 2000). Also, PORCN lacks the ATP-binding cassette that is characteristic of ABC transporter (Galli et al., 2007). The function of PORCN targets WNT to the ER membrane, facilitating its modification also may have an effect on WNT conformation (Ashcroft and Roper, 1993). One major function of WNT palmitoyleation by PORCN is to promote protein-membrane association (Guan and Fierke, 2011). Since PORCN is unable promote lipid-modification of the C93/C77 mutants, it is likely that 
PORCN regulates lipid-modification of the S224/S209 sites of WNT1 and WNT3A as well. The observation that PORCN can also promote the modification of WNT1, the lipid modification of C93 is PORCN-independent while that of S224 is PORCN-dependent (Zhai et al., 2004; Galli and Burrus, 2009). It is unclear how modified WNT proteins could be altered. It's critical to understand what modification that secreted WNT proteins undergo to understand the initiation steps of cancer. 


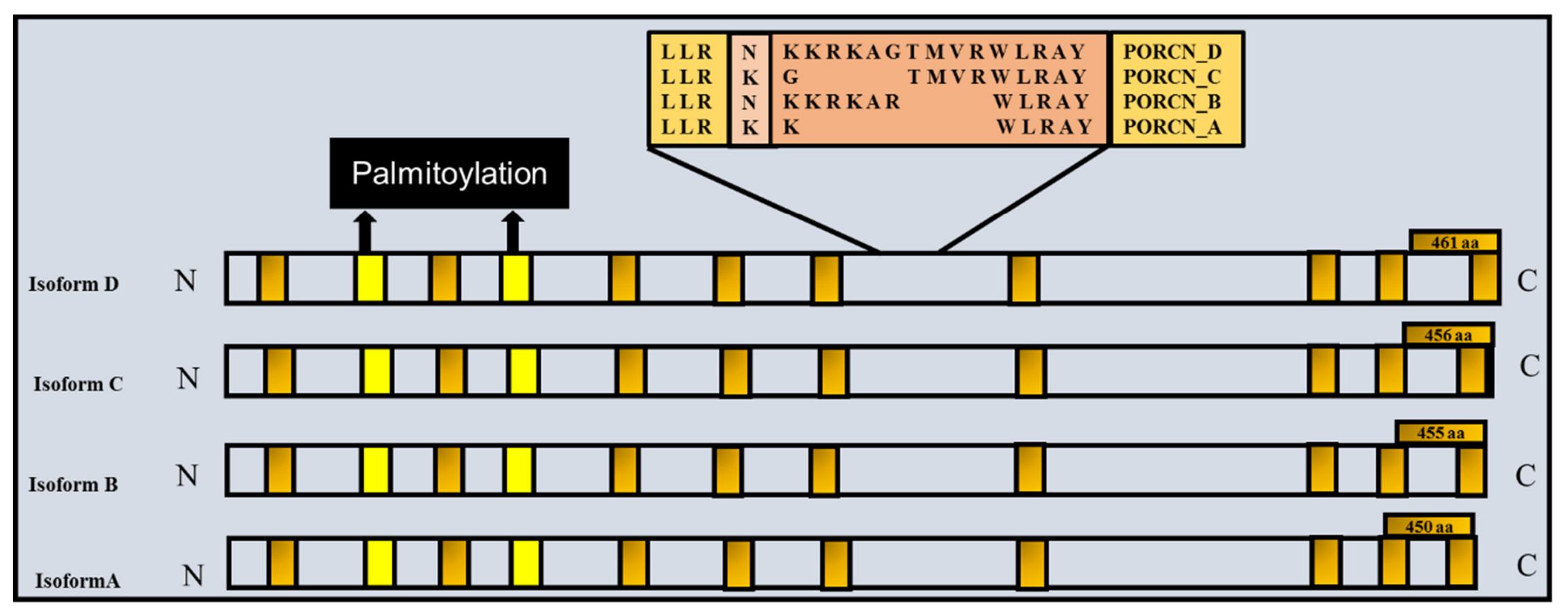

Figure 1.6: Domain organization of PORCN. The three-dimensional structure of all three PORCN is not known (a) PORCN isoforms are approximately $51 \mathrm{kDa}$ and palmitoyleation sites (yellow). It has nine transmembrane (TM) domains (gold). There are four isoforms of PORCN (A, B, C and D) that differ by 5-11 amino acids within the third cytoplasmic loop (shown in insert). It has two sites of palmitoylation (yellow), and the carboxy-terminal tail that is predicted to be embedded within the phospholipid bilayer. The TM domains are defined by the following amino acids: $29-47,67-89,96-115,155-177,197-219,250-271,343-360$, and 395-417. 


\subsubsection{Palmitoyleation of WNT}

Palmitoleation is a reversible post translational modification (PTM) that is crucial in the communication of proteins with other cellular components (Guan and Fierke, 2011). It plays a role in cell-surface communication, where many palmitoleated ligands and voltage-gated channels become associated with multiple intracellular membranes and plasma membranes (Guan and Fierke, 2001). Moreover, the attachment of the fatty acids to proteins has been found to be important for functions such as targeting proteins to the membrane and subcellular protein trafficking (Linder, 2001). Palmitoyleation and palmitoleation also allow proteins to not only have an affinity to the cell membrane, but also to other cellular compartments, such as the ER or Golgi apparatus (Rios-Esteves and Resh, 2014). Palmitoyleation is the attachment of palmitic acid to cysteine or serine and threonine protein residues. The covalent attachment of fatty acids happens more frequently at cysteine residues, resulting in S-palmitoyleation, whereas palmitoyleation at serine and threonine residues yields an O-palmitoyleation (Guan and Fierke, 2001). Palmitoyl acyltransferases (PAT) are enzymes involved in catalyzing the palmitoyleation of proteins (Guan and Fierke, 2001). There are 23 versions of PATs found in mammals, which regulate the majority of protein palmitoyleation present in mammalian cells (Guan and Fierke, 2001). Palmitoyleation of the WNT and Hedgehog proteins are critical for maintaining their physiological functions (Clevers, 2006). Palmitoyleation of WNT is critical for the protein's ability to bind to its cell surface FzD receptors and initiate downstream signaling events leading to stabilization of $\beta$-catenin (Linder, 2001). The palmitoyleation of proteins is performed by PORCN, which is an ER bound o-acyltransferase (Van Den Heuvel et al., 1993). Palmitoleic acid and other fatty acids with 13-16 carbon lengths are linked onto 
Ser209 of WNT3A (Gao and Hannoush, 2014). Despite its physiological importance, the biochemical mechanism governing WNT acylation by PORCN is poorly understood. In all, there is an unusual $O$-acyl modification with palmitoleic acid (9-cis-hexadecenoic or 9-16:1) at a conserved serine residue (Main and Fuller, 2021). Following synthesis, WNT proteins are processed in the ER, where the conserved signal sequence is cleaved before glycosylation and fatty acylation. Following desaturation of palmitoyl-CoA to generate a dedicated pool of palmitoleoyl-CoA in the ER by stearoyl-CoA desaturase 1 (SCD1), the subsequent $O$-acylation requires an $O$-acyltransferase by PORCN (Fig. 1.7) (Hosseini et al., 2019). The specificity of the reaction is due to a structural feature in the enzyme that will only permit the use of the cis-9-monoenoic fatty acid; only those WNT proteins esterified in this way can separate from the PORCN-WNT complex (Hosseini et al., 2019). The $O$-palmitoleoyl residue is essential for intracellular trafficking and activity of WNT proteins within cells and is crucial for maintaining their structural integrity and assists their secretion by facilitating movement from the trans Golgi network to the plasma membrane in signalling cells (Mikels and Nusse, 2006). The transport mechanism involves transfer to the co-chaperone 'WNTless' or 'WLS' (G protein-coupled receptor 177 (GPR177)), a conserved membrane protein that binds to the palmitoleoyl residue of WNT proteins and facilitates transport by vesicular or non-vesicular means to the plasma membrane and then across it (Nygaard, et al., 2021). In addition, serine acylation may be important for extracellular long-range transport of WNT proteins in lipoprotein particles or with protein chaperones (Takada et al., 2006). To initiate signaling, WNT proteins bind to cell-surface receptors of the FzR family, where the palmitoleoyl residue fits neatly within a hydrophobic cleft to which it binds to enable ligand-receptor interactions (Mikels and 
Nusse, 2006). WNT proteins are deactivated by an extracellular carboxylesterase ('Notum'), which removes the palmitoleic acid group (Kakugawa et al., 2015). WNT proteins are relatively hydrophobic and are stabilized in the aqueous environment of the cell by binding in a hydrophobic space created by specific glypicans, i.e. heparan sulfate proteoglycans that are bound to the outer surface of the plasma membrane by a glycosylphosphatidylinositol anchor. These serve as a reservoir from which WNT proteins can be handed over to signalling receptors (FzD) (Clevers, 2006). 


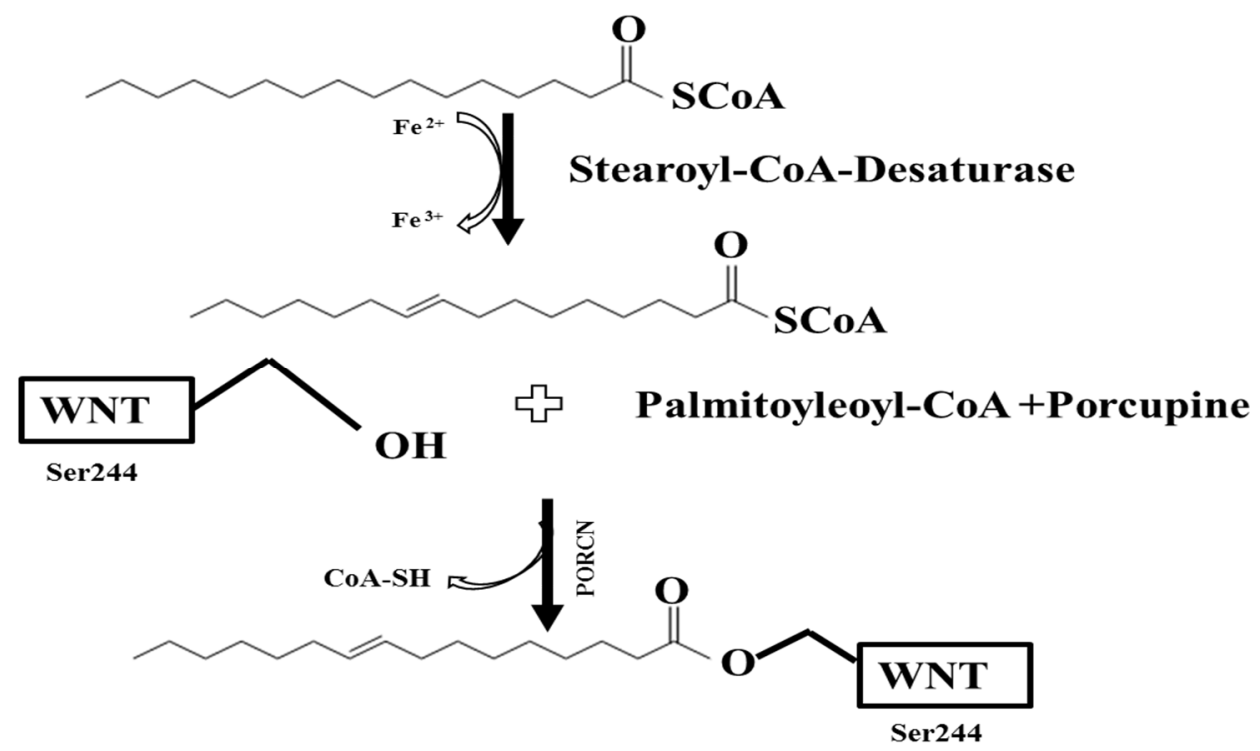

Figure 1.7: A schematic model illustration how WNT gets palmitoyleated by Porcupine (PORCN). PORCN is palmitoyleated at residue Cys187 Lipid modification of PORCN then regulates the acylation level of WNT3A. PORCN is fatty acylated via a thioester linkage. PORCN recognizes fatty acid Coenzyme A (CoA) substrates that have 13-16 carbon atoms including palmitoleoyl-CoA and acylates newly synthesized WNT3A in the ER at conserved Ser209. Cys77 residues on WNT3A are not lipid modified. PORCN fatty acylation (at Cys187) adjusts the level of WNT3A palmitoyleation and its signaling activity. The O-palmitate bound to WNT3A does not undergo rapid cycles of acylation and deacylation during the lifetime of the protein. Palmitoyleation of WNT3A neither requires glycosylation nor protects WNT3A from degradation in the proteasome, and it is required for maximal WNT3A secretion. Monoglycosylation of palmitoylated WNT3A occurs in the ER-Golgi system and sets it routes to secretion. Mature WNT3A traffics post-Golgi which acts as export sites for its secretion on exosomes. PAT, palmitoyl acyltransferase 
(adapted from Rios-Esteves and Resh, 2014; illustration using ChemDraw tool by R. Mohamed). 


\subsubsection{Evi-WNTLESS (WLS) the WNT chaperone}

WLS or Evenness Interrupted/Sprinter in Drosophila, MOM-3/Mig-14 in $C$. elegans, and GPR177 in mammals) is an evolutionarily protein and putative orphan Gprotein coupled receptor (GPCR) (Port et al., 2011). WLS is an integral membrane protein predicted to have eight transmembrane (TM) helices, with an extended luminal loop that has been shown to be essential for WNT binding (Fig. 1.9), and an ER recycling motif at the cytoplasmic $\mathrm{C}$ terminus It consists of four transmembrane domains with a large $\mathrm{N}$ terminal globular extracellular/luminal domain that has two potential N-linked glycosylation sites (Fu et al., 2009) (Fig. 1.8). It is involved in regulation of expression, subcellular localization, and binding and organelle-specific association of WNT proteins (Port et al., 2011). The essential role of WLS is establishment of the anterior-posterior body axis formation during development for the secretion of multiple WNT proteins to regulate WNT proteins sorting and secretion in a feedback regulatory mechanism (Belden and Barlowe, 2001). WLS genomic locus is composed of three exons with two possible splice variants to encode novel proteins of 594 (the longest isoform A) amino acids and molecular weight of $60 \mathrm{kDa}$ (Fu et al., 2009). WLS has been found to be localized in the entire WNT secretory route including ER, Golgi, vesicles and plasma membrane (Belden and Barlowe, 2001). The structure of WLS is characterized by having a lipocalin-like structure that allows WLS to bind to hydrophobic regions, like the palmitate groups, in mature WNTs (Port et al., 2011). The affinity of WLS toward the hydrophobic regions in WNTs might facilitate the physical association of WNT with WLS (Coombs et al., 2010). WLS binds WNTs in the ER, cycles to the plasma membrane, and then returns to the ER through the Golgi. An ER-targeting sequence was identified at the carboxyl terminus of native WLS 
that is critical for ER retrograde recycling and contributes to WNT secretory function (Xhang et al., 2009). In addition, loss of GPR177 in mice clearly affects the canonical WNT signaling pathway as suggested by the depletion of active $\beta$-catenin (Zhang et al., 2009). The glycosylation site is found S239 and essential for the function of the protein and required for WNT secretion (Ikonen, 2001). Mutations in the WNT palmitoleation site, inhibition of PORCN, and downregulation of WLS expression prevent WNT ER exit and secretion (Moti et al., 2019; Takada et al., 2006; Yu et al., 2014). Similarly, whole-body knockout (KO) of WLS is lethal in mice (Fu et al., 2009), and tissue-specific KO models display various WNT-related phenotypes (Carpenter et al., 2010; Zhong et al., 2012). Thus, the PORCN-WLS acylation-secretion module is required and essential for all WNT functions. The role of WLS in this process remains unclear. In the journey from the ER to the plasma membrane, the WNT-WLS complex traverses the Golgi apparatus, where additional WNT glycosylation occurs, to reach the plasma membrane. WNT glycosylation (Fig. 1.9) might determine differential targeting of the WNT-WLS complex to different exit sites on polarized cells (Yamamoto et al., 2013). The covalently attached Opalmitoyleation (O-PAM) restricts how WNTs travel after they reach the cell membrane, where they must be transported by one of several forms of facilitated diffusion to reach their receptors on neighboring cells. The WNT-WLS complex might transfer WNTs to cell-surface proteoglycans, soluble transport proteins, cytonemes, or exosomes (McGough et al., 2020; Routledge and Scholpp, 2019). Ultimately, in a step that is again dependent on the presence of the O-PAM, WNTs are delivered to FzD and its associated co-receptors on the surface of the receiving cells to initiate downstream signaling (Niehrs, 2012; Yu and 
Virshup, 2014). WLS can then recycle from the plasma membrane back to the ER for additional cycles of WNT transport (Belenkaya et al., 2008; Yu et al., 2014). 


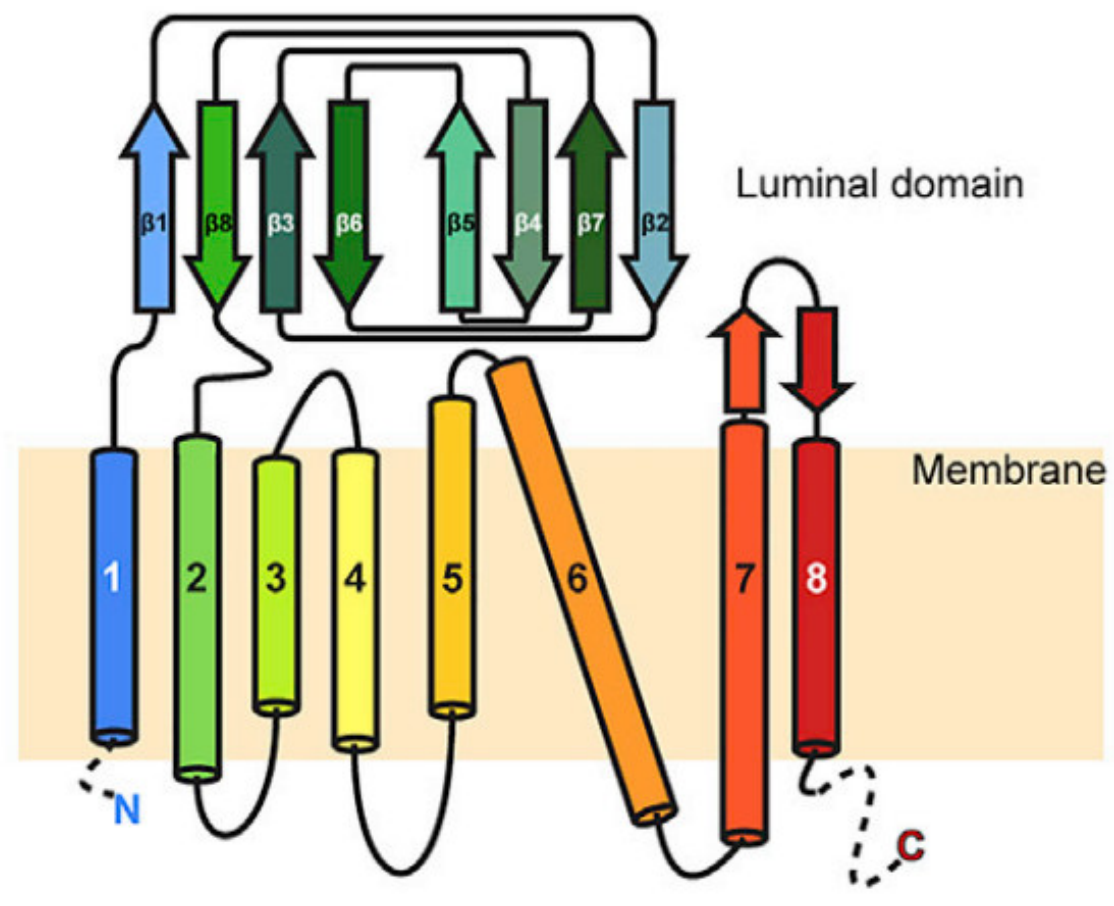

Figure 1.8: WLS is a $62-\mathrm{kDa}$ protein that is proposed to have seven or eight TM domains (5 and 6) and one glycosylated site between site 1 (blue) and 2 (green) (adapted from Nygaard et al. 2020). 


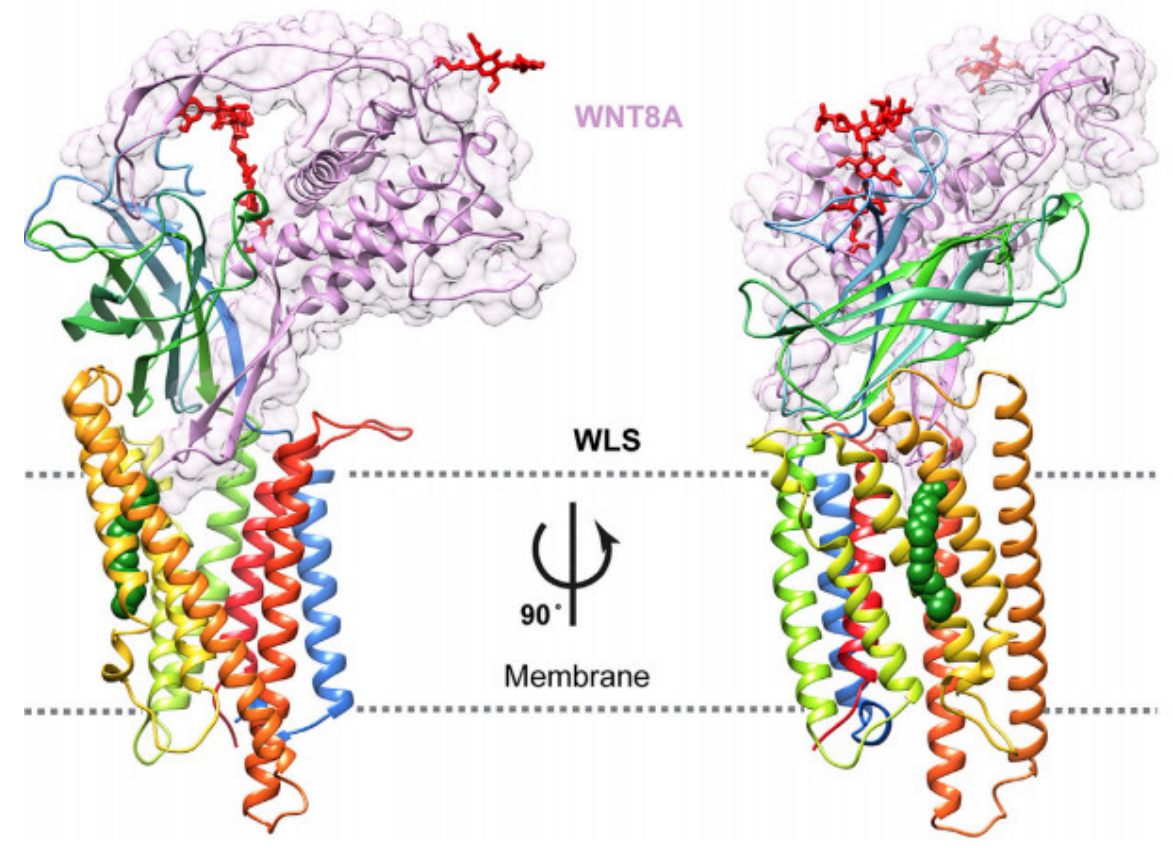

Figure 1.9: The structure of WNT8A bound to WLS, with WLS colored in rainbow and WNT8A in violet with a transparent surface. The PAM is represented as green spheres extending out between TM helices 4 and 5. Glycosylation of WNT8A at two sites is shown in red, represented as sticks. (Nygaard et al., 2020). 


\subsubsection{Glycosylation of WNT}

WLS is a multipass transmembrane protein thought to principally act as cargo receptor that ferries WNTs along their exocytic pathAs a major post-translational modification (PTM), glycosylation plays a vital role in the folding, stability, subcellular localization and biological functions of glycoproteins (Ikonen, 2001). In addition, Glycosylation has been widely recognized as an important hallmark of cancer and significantly correlates with the development, progression, metastasis, and chemoresistance of tumors (Tang et al., 2012; Jones et al., 2018).

Protein glycosylation includes the addition of N-linked glycans, O-linked glycans, phosphorylated glycans, glycosaminoglycans and glycosylphosphatidylinositol (GPI) anchors to peptide backbones and C-mannosylation of tryptophan residues (Very et al., 2018). Most of the glycosylation takes the form of N-glycan which is found in most living organisms (Very et al., 2019). The role of N-glycan is very important since carbohydrates play in achieving the proper conformation folding of glycoproteins and modulate thermostability of proteins and their overall charge (Dennis et al., 2009). The WNT signaling pathway is highly modified before secretion (Arey and López, 2001). When WNT reaches the ER, it undergoes important post-translation modification (PTM) (Nusse, 2006). First, the oligosaccharyl transferase complex (OST) rapidly and efficiently attaches $\mathrm{N}$-linked oligosaccharide chains to the appropriate residues on the peptide backbone (Asn108 and Asn414 in Wg) (Tanaka et al., 2002). However, the biological function of WNT N-glycosylation remains unclear. WNT members vary in the number and position of glycosylation sites and the roles of glycosylation also seem to be unconserved within the WNT family. It has been demonstrated that replacement of all N-glycosylation sites does 
not affect WNT1-induced autocrine or paracrine signaling in several cellular contexts, indicating that glycosylation is not essential for either secretion or signaling (Riggleman et al., 1990). However, in the case of WNT3A and WNT5A, glycosylation precedes lipid modification and is important for WNT secretion but not for WNT actions (Kurayoshi, et al., 2007). Most available data regarding the activities of WNT lipidation and Nglycosylation were obtained from cell-based assays (Mason et al., 1992; Zhai and Chaturvedi, 2004). WLS has been shown to be localized in the ER, Golgi apparatus and on the plasma membrane (Metcalfe and Bienz, 2011; Takada et al., 2006). After released from the cell surface, WNT proteins reach their receiving cells by a facilitated movement involving lipoprotein particles and heparan sulfate proteoglycans (HSPG; Dally and Dlp in Drosophila) (Belden and Barlowe, 2001; et al., 2006). Various studies have indicated that WNTs can be N-glycosylated, in which multiple asparagine residues of WNT proteins are appended with N-linked oligosaccharide chains (Fig. 1.10) (Ikonen, 2001). Furthermore, inhibiting protein glycosylation will trigger ER stress (Shaheen, 2018). The ER is a dynamic network will affect protein synthesis and folding (Schwarz and Blower, 2016). In tumour cells, the protein-processing machinery in the ER is constantly challenged by either intrinsic stresses, such as oncogenic activation and point mutation, or extrinsic perturbations, such as hypoxia, nutrient deprivation and acidosis (Fouad and Aanei, 2017). All of these would alter protein homeostasis and lead to the accumulation of misfolded proteins in the ER lumen, a state known as ER stress (Shaheen, 2018).

Post-translational modification such palmitoyleation and glycosylation are regulated by cellular responses. Stressors can activate both proteolytic and non-proteolytic protein factors, which can ultimately alter signalling pathways (Jones and Kemp, 2008). 
The aberrant activation of signalling pathway such WNT under different stressors could the key to understand how cancer activations at early stages of the disease. 


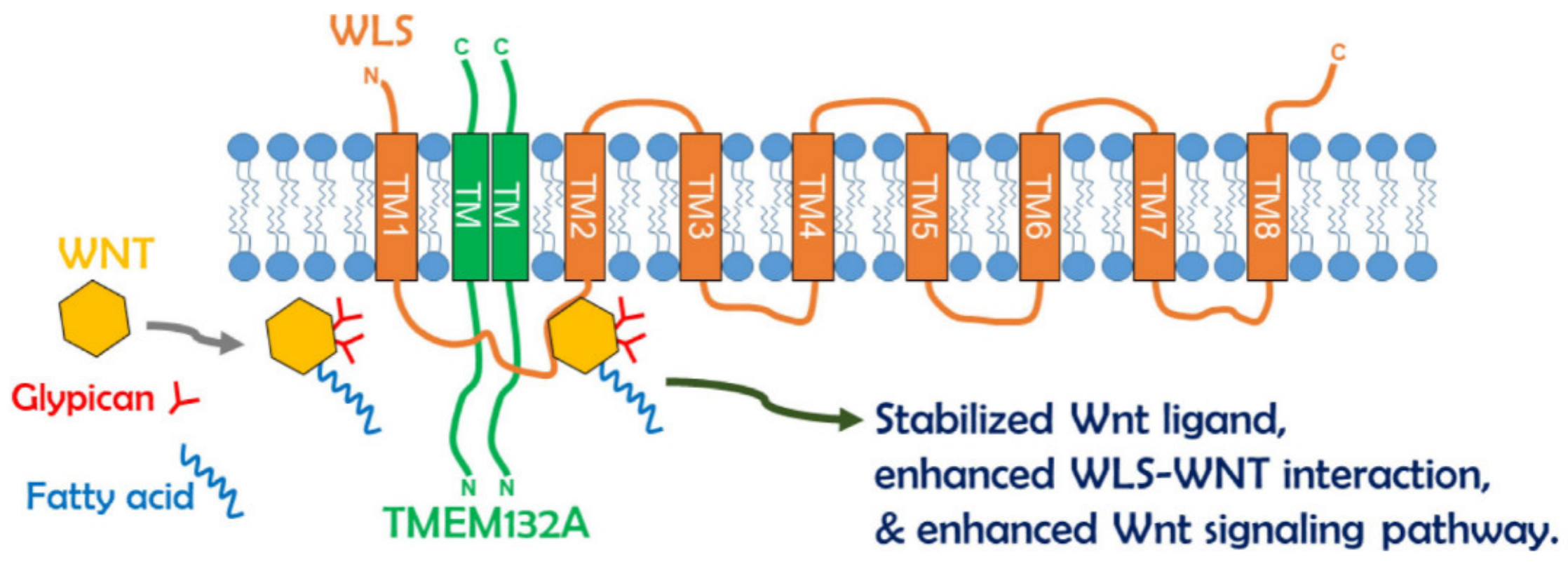

Figure 1.10: Illustration showing the WNT ligand gets chaperoned by protein WLS. WLS is eight transmembrane (TM) protein. Nglycosylation of WLS will increase its affinity for WNT binding, allowing for WNT stabilization and enhanced WNT secretion to activate the WNT signalling pathway. TMEM 132A is a transmembrane protein 132A (adapted from Li and Niswander, 2020). 


\subsection{The tumor microenvironment}

\subsubsection{Endoplasmic reticulum stress}

The endoplasmic reticulum (ER) is the site of synthesis and folding of membrane and secretory proteins, which, collectively, consume a large part of the total protein output of a mammalian cell (Jan et al., 2014). Therefore, the protein alteration through the ER must be carefully observed for abnormalities, including the buildup of misfolded proteins (Fu and Hayer-Hartl, 2009). Mammalian cells have evolved a unique set of signaling pathways from the ER to the cytosol and nucleus, to allow the cells to respond to the presence of misfolded proteins within the ER (Jan et al., 2014). Physiologic stresses, such as increased secretory load, or pathological stresses, such as the presence of hypoxia, that can lead to an imbalance between the demand for protein folding and the capacity of the ER for protein folding, results in ER stress (Bernales et al., 2006). To recognize ER stress, eukaryotic cells have evolved a group of signal transduction pathways, collectively termed the unfolded protein response (UPR). The UPR is a complex process that is initiated by the activation of the three UPR stress sensors: inositol-requiring protein 1 (IRE1), protein kinase RNA-like ER kinase (PERK) and activating transcription factor 6 (ATF6). These sensors control adaptive process through both transcriptional and non-transcriptional responses, affecting almost every aspect of the secretory pathway, including protein folding, ER biogenesis, ER-associated degradation (ERAD), protein entry to the ER, autophagy and secretion, among others (Calfon et al., 2002; Yoshida et al., 1997; Lee et al., 2003). ER stress may be caused by factors that impair protein glycosylation or disulfide bond formation, or by overexpression of or mutations in proteins resulting cell death (Lin et al., 2008). The role of the UPR in influencing cellular life/death decisions, in response 
to ER stress, has led to intense interest in the link between UPR signaling and human diseases that arise from pathologic conditions eliciting ER stress (Jan et al., 2014). ER stress simultaneously activates UPR to reduce protein synthesis, degrade misfolded proteins, and produce molecular chaperones (Zhang et al., 2010). PERK, IRE1, and ATF6 are ultimately responsible for the activation of a set of transcription factors through a complicated and nonparallel process. (Jiang et al., 2016). Common transcription factors known to be activated during stress include Glucose Regulatory Protein 78 (GRP78); Hypoxia-Inducible Factor 1 alpha (HIF1 $\alpha$ ); Metallothionein (MT1), Nuclear Factor Erythroid-derived 2-Related factors 1 and 2 (NFE2L1 and NFE2L2).

1.8.1.1 WNT secretion and endoplasmic reticulum stressors

WNT secretion and ER stress are two fundamental biological processes that participate in diverse biological and pathophysiological of cancers and diseases. Interestingly, WNT secretion takes place at the ER, however there is less known about the link shared between WNT secretion and ER stressors (Mohamed et a., 2021). The majority of studies focused on how stressors are involved in the WNT/ $\beta$-catenin pathway (van Lidth de Jeude et al., 2017). Previous studies showed that ER stress could promote the degradation of $\beta$-catenin, likely through impairing the WNT signaling pathway upstream of GSK-3ß (Shi et al., 1998). Other studies identify a mitochondria-WNT signaling axis through which a decrease in mitochondrial ATP reduces calcium uptake into the ER, leading to ER stress and to impaired WNT signaling (Costa et al., 2019). Furthermore, it has been reported that deletion of p24 genes activates an ER stress response (Takada et al., 
2006). The p24 family of proteins controls WNT secretion by regulating the ER export of WNT and acetyltransferase PORCN is involved in Wg secretion by regulating the posttranslational modification of Wg which concluded that unsecreted WNT triggers ER stress (Kim et al., 2015). It is unknown which intermediates are involved during ER stressors that triggers unsecreted WNT proteins. Stress is activated and triggers the UPR, which restores homeostasis or activates cell death. This also protects tumour cells from apoptosis in conjunction with induced tumour dormancy and permitting regrowth of the tumour when favorable conditions have been restored (Costa et al., 2019). In one study, WLS dysfunction-induced ER stress buffers the toxicity of unsecreted WNT during development, while the detailed mechanisms need to be further addressed (Verras et al., 2008). It would be interesting to determine if unsecreted WNT-induced ER stress and is a key factor in development of diseases. Moreover, understanding PORCN and WLS response to stress would be a promising field of study (Dhanvantari and Loh, 2000). There is a gap in knowledge of how direct ER stressors can influence PORCN and WLS components of the WNT signalling pathway and if they play a role in WNT secretion during ER stress.

\subsubsection{Hypoxia}

The inability to meet cellular oxygen demands is called hypoxia, which results in the activation of specific cellular stress responses (Connet et al., 1985). The normal oxygen concentration in humans ranges between approximately $9.5 \% \mathrm{O}_{2}$ in the renal cortex to $4.6 \%$ $\mathrm{O}_{2}$ in the brain, with neurons being extremely sensitive to hypoxia (Thomlinson et al., 1955). Cellular stress is likely a part of a broader, complex response to the hypoxic 
condition (Ding et al., 1998). Stress proteins undergo modification that could be important in regulating the response to hypoxic injury in tissues and organs (Ziello et al., 2007). Hypoxia induces a number of complex intracellular signaling pathways, such as hypoxiainducible factor (HIF) pathway (McGarry et al., 2019). Hypoxia can disrupt the normally oxidative environment within the ER, leading to protein misfolding (Chipurupalli, et al., 2019). Hypoxia is a potent trigger of PERK signaling in cultured mammalian cell lines (Semenza, 2010). Hypoxia develops frequently in solid tumors, as rapid cancer cell proliferation outpaces the ability of the vasculature to deliver oxygen (Rouschop et al., 2013). The presence of hypoxia in cancer has significant clinical implications, including resistance to chemo- and radiotherapy, increased likelihood for metastases, and worse prognoses (Chipurupalli, et al., 2019). Hypoxic stress induces gene expression changes in order to protect, adapt, and survive by altering the cell's metabolic and angiogenic pathways and restoring oxygen homeostasis (Bartoszewski et al., 2011). A major mechanism mediating adaptive responses to hypoxia is the transcriptional program activated by hypoxia-inducible factor 1 (HIF-1) (Semenza, 2010). HIF is heterodimeric DNA-binding complexes consist of two basic helix-loop-helix proteins (Paul et al., 2010). HIF- $1 \alpha$ or HIF- $2 \alpha$. In hypoxia, the $\alpha / \beta$ heterodimer binds to a core penta-nucleotide sequence (RCGTG) in the hypoxia response elements (HREs) of target genes HIF- $\alpha$ subunits have a very short half-life. Cells continuously synthesize and degrade HIF- $\alpha$ protein (Weidemann, A., Johnson, 2008). However, under decreasing concentrations of oxygen, the degradation of HIF- $\alpha$ is delayed (Freedman et al., 2002). The connection between oxygen and the HIF- $\alpha$ subunit is provided by distinct enzymatic reactions: the 
hydroxylation of two prolyl residues (Pro402 and Pro564 in human HIF-1 $\alpha$ ) in the oxygendependent degradation domain (ODDD) of the $\alpha$-subunits (Strowitzki et al., 2019).

Under hypoxic conditions, prolyl hydroxylation is suppressed and HIF- $\alpha$ proteins breakout from proteasomal destruction and can accumulate (Freedman et al., 2002). The heterodimeric activating complex HIF then binds to the HRE in promoter or enhancer sequences of target genes HIF-1 $\alpha$ controls metabolic and pH-regulating pathways (Strowitzki et al., 2019). Cells respond to hypoxia by HIF-1 $\alpha$-mediated upregulation of glucose transporters (Glut-1 and Glut-3), as well as the enzymes of glycolysis (Nagao et al., 2019). Hypoxia alters cancer cell metabolism and contributes to therapy resistance by inducing cell quiescence. Hypoxia stimulates a complex cell signaling network in cancer cells. Hypoxia regulates tumor neovascularization, metabolism, cell survival, and cell death (Strowitzki et al., 2019).

\subsubsection{WNT secretion and hypoxia}

Hypoxia is observed when a non-physiological level of oxygen is restricted or below the normal levels, a state common in a majority of malignant tumours (Tabas and Ron, 2011). Significantly proliferating and expanding tumor tissue, oxygen demand is surpassed by oxygen supply (Muz et al., 2015). Cells respond to these stresses through the induction of both HIF-1-dependent and HIF-1-independent mechanisms (Vincent et al., 2000). A study reported that tumour hypoxia blocks WNT secretion through induction of ER stress. The biological significance could be implicated as a loss of $\beta$-catenin stability in hypoxic cells (Denko et al., 2003). Hypoxia may interfere with the machinery 
responsible for the post-translational modifications and therefore reduce the efficiency of WNT secretion (Verras et al., 2008). Hypoxia has been shown to generate ER stress through the accumulation of misfolded proteins (Tu et al., 2000). Proteins with incorrect disulfide bonds accumulate in the ER until they undergo retrograde transport to the cytoplasm for proteasomal degradation (Haynes et al., 2004). Hypoxia, combined with acidosis, inhibits WNT-induced transcriptional activity of endogenous Axin2, a common WNT target (Verrase et al., 2008). Another study found that upregulation of WNT signalling under hypoxia promotes lung cancer progression (Hong et al., 2017). However, we can predict that WNT secretion is potentially increased in order for the signalling to be activated which leads to $\beta$-catenin accumulation. In this study they highlighted the role of hypoxia inducible factor 2 alpha (HIF-2 $\alpha$ ) is the major effector of lung cancer cells in WNT signaling activation under hypoxia and crosstalk between WNT pathway and hypoxia participates in cancer progression (Hong et al., 2017). It was also noted that knockdown of HIF- $2 \alpha$ decreases $\beta$-catenin expression and inhibits hypoxia-induced cell migration and invasion (Hong et al., 2017). The question that needs to be addressed is whether the involvement of HIF-1 $\alpha$ and the ER stressors in activating WNT signaling under hypoxia, could result in promoting the survival and malignancy of colon cancer cells.

\subsubsection{Oxidative stress}

Reactive oxygen species (ROS), also known as reactive oxygen intermediates (ROI), are byproducts of normal cellular metabolism (Paiva and Bozza, 2014). Low and moderate amounts of ROS have beneficial effects on several physiological processes including killing of invading pathogens, wound healing, and tissue repair processes 
(Puertollano et al., 2011). However, antioxidant pathways can limit the adverse effects of ROS, their levels can be stimulated by many oxidative stressors and maintained such that they contribute to tissue damage (Paiva and Bozza, 2014). ROS are produced in response to air pollutants, tobacco smoke, ionizing and nonionizing radiations, foods and drugs, as well as xenobiotics, chemical agents like quinones, heavy metals such as lead, arsenic, mercury, chromium, and cadmium; organic solvents; and pesticides are common exogenous sources of ROS (Bhattacharyya et al., 2014; Bolton et al., 2000). On the other hand, endogenous sources could contribute the generation of ROS in the intracellular compartments including mitochondria, the endoplasmic reticulum, peroxisomes, nuclei, the cytosol, plasma membranes, and even extracellular (Bhattacharyya et al., 2014). The mitochondrial electron transport chain is the major site of ROS production in most mammalian cells (Bhattacharyya et al., 201). Enzymes that catalyze ROS-generating chemical reactions are peroxidases, NADPH oxidase, NADPH oxidase isoforms (NOX), xanthine oxidase (XO), lipoxygenases (LOXs), glucose oxidase, myeloperoxidase (MPO), nitric oxide synthase, and cyclooxygenases (COXs) spaces (Bhattacharyya et al., 2014; Balaban et al., 2005; Swindle et al., 2007). Both sources endogenous and exogenous are capable of disruption of normal cellular homeostasis by redox signaling contributes to disease in virtually every organ including the development of cancer (Bhattacharyya et al., 2014).

\subsubsection{WNT secretion and oxidative stress}

There is a growing concern to manage the incidence of internal stresses in the human body. Disruption of normal cellular homeostasis by redox signaling may result in 
cardiovascular, neurodegenerative diseases and cancer (Fig. 1.11) (Wang et al., 2013). The presence of cysteine rich regions in the WNT pathway could potentially function as a redox switch (Daub et al., 1998). Since WNT is modified by PORCN and WLS, we can conclude that they are targets of oxidation and ER stressor as well (Mohamed et al., 2021). The underlying mechanism of ROS can be a key effector in the activation of WNT secretion. Previous work investigated oxidative stress that resulted in abnormal WNT signaling activity in human retinal microvascular endothelial cells, which may be a major contributor to retinal neovascularization. The study reported that oxidative stress upregulates WNT signaling in human retinal microvascular endothelial cells through activation of disheveled causing tube formation and cell migration (Zhang et al., 2020). Enzymes such as xanthine oxidase are mainly expressed in the liver and small intestinal mucosa within the GI tract (Van Der Vliet, et al., 1998). The reaction happens when in contact with oxygen which catalyzes oxidation of hypoxanthine to xanthine and then, to uric acid during purine catabolism (Harris et al., 1999). WNT secretion relies on lipid modification and reaction superoxide is generated during oxidation of hypoxanthine to xanthine which leads us to question whether these molecules could interfere with WNT secretion. Previous studies have focused on oxidative stress and how it modulates the function of $\beta$-catenin. Interestingly, low levels of oxidative stress stimulate the interaction of $\beta$-catenin and activate various transcription factors (Essers et al., 2005). Binding of $\beta$-catenin strongly enhances activity of transcription factors which leads to a protective response that inhibits cell cycle progression and which results in oxidative damage. However, there are also benefits with increased levels of ROS which allows to stimulate cell proliferation and transformation (Young et al., 2013). Also, ROS are now considered to be second 
messengers in signal transduction pathways. Often important in cell development WNT/ $\beta$ catenin signaling is upregulated in inflammatory processes and oxidative stress and in many cancers, although there are some exceptions for cancers. Cancer cells have high levels of ROS intermediates (Arnold et al., 2001). An intriguing possibility is that the higher levels of ROS found in cancer cells increases WNT secretion, resulting in increased $\beta$-catenin accumulation which allows cells in damaged tissues to rapidly proliferate. 


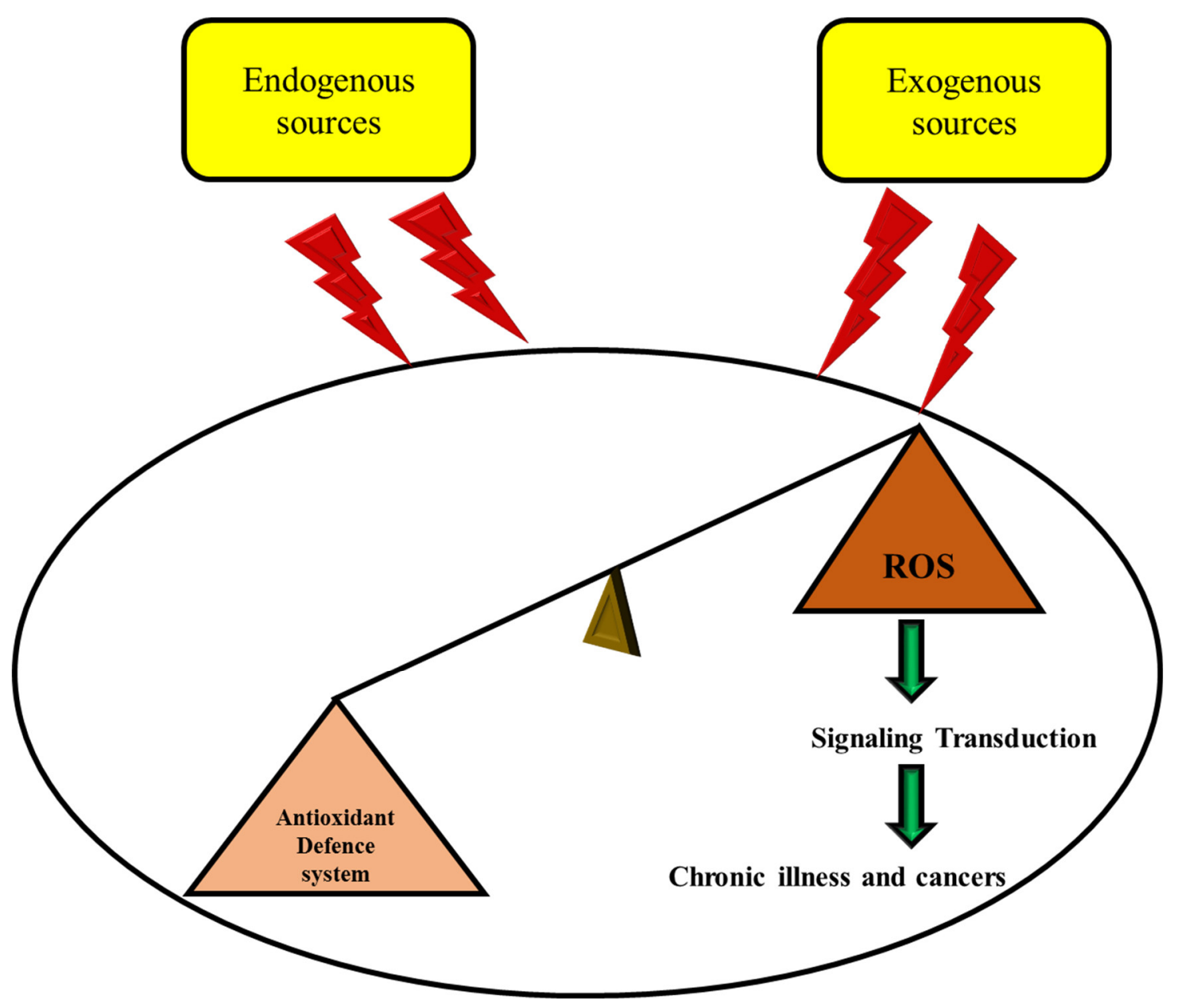

Figure 1.11: Illustration of the sources of stressors and their effect on the balance of antioxidant system. Exogenous and endogenous sources of stressors could result in an increase in ROS which lead to deregulation of cellular signaling will results in chronic illness and cancer. 


\subsection{Stress induced transcriptional activation factors}

Different types of environmental and physiological stress are a constant challenge to all living organisms. To adapt with the deleterious effects of stress, cells have developed rapid molecular responses to repair the damage and protect against further exposure to the same and other forms of stress (Fulda et al., 2010). The best-characterised response is the synthesis of number transcription factors. These transcription factors can result in transcriptional mechanisms can play an important role in the regulation of the stress response. In (Fig. 1.12), the unfolded protein respond is responsible activated during stress in different parts of the human body. For our interest we focued on a number of transcription factors are regulated under different stressors, including GRP78, HIF1 $\alpha$, MT1, NFE2L1 and NFE2L2. 


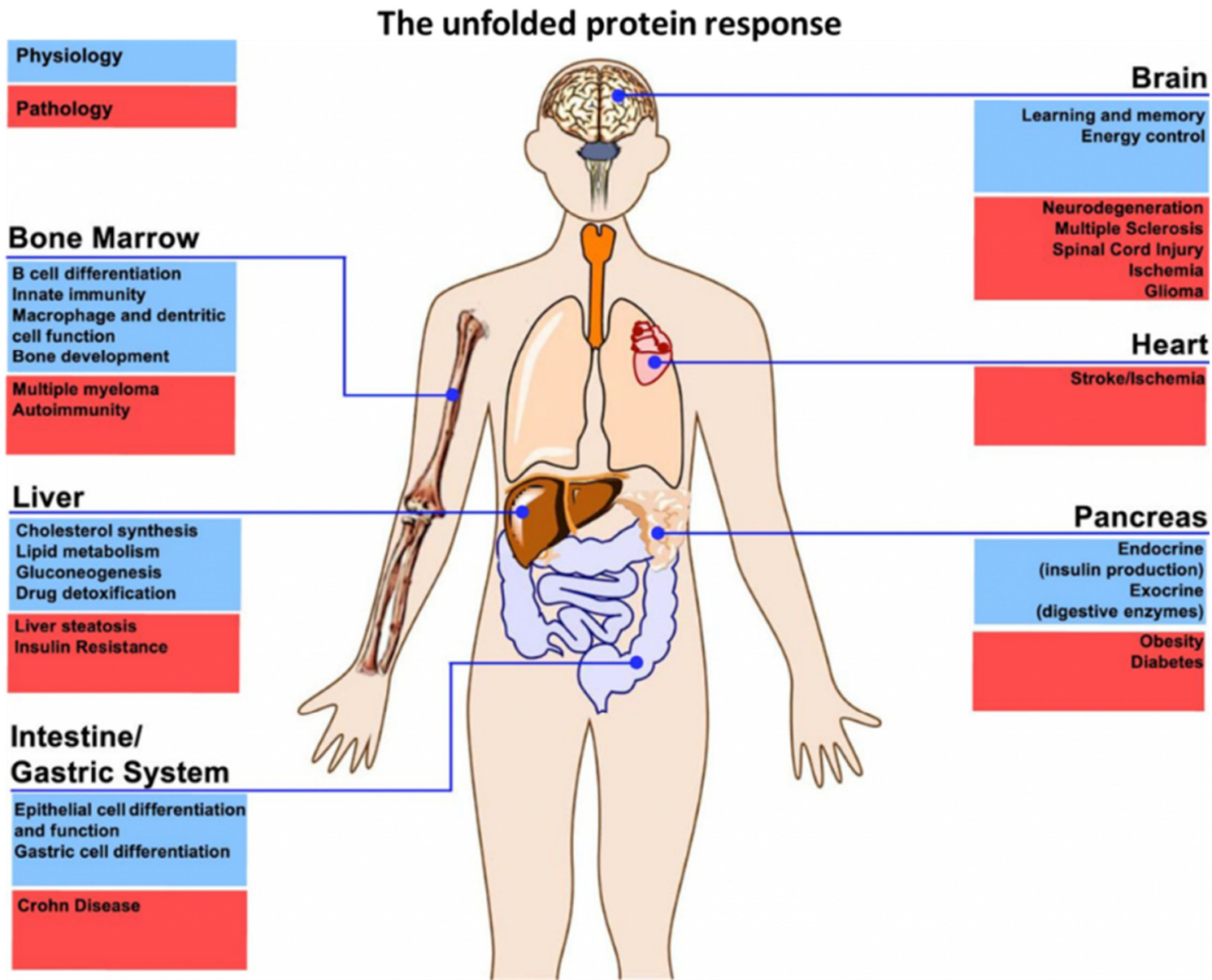

The unfolded protein response has important physiological functions. ER stress and disruption or dysregulation of the unfolded protein response has been implicated in a wide variety of diseases.

Figure 1.12: Illustrates the crucial role of UPR in all the human tissues and organs could results from hypoxia and oxidative stressors. UPR effect wide range of physiological and pathological outcomes in many chronic illnesses and diseases. http://www.train$\underline{\text { ers.eu/background/ }}$ 


\subsubsection{Glucose-regulated protein 78 (GRP78)}

GRP78/BiP is a major endoplasmic reticulum (ER) chaperone protein critical for protein quality control of the ER, as well as controlling the activation of the ERtransmembrane signaling molecules (Wang et al., 2009). The function of GRP78 is obligatory for early embryonic development. However, in adult animals, GRP78 is preferably required for cancer cell survival under pathologic conditions such as tumor progression and drug resistance (Koumenis, 2006). GRP78 is a master regulator for ER stress because of its role as a major ER chaperone with antiapoptotic properties, as well as its ability to control the activation of UPR signaling (Pfaffenbach, et al., 2011). The interaction of GRP78 with the UPR-induced signaling and apoptotic pathways is Upon ER stress, GRP78 is released from ER transmembrane signal transducers, including PKR-like ER kinase (PERK), inositol-requiring enzyme 1 (IRE1), and activating transcription factor 6 (ATF6), leading to the activation of these UPR signaling pathways (Pfaffenbach, et al., 2011). After the disassociation from GRP78, PERK dimerizes to promote its autophosphorylation and activation (Wang et al., 2009). Activated PERK phosphorylates the eukaryotic translation-initiation factor $2 \alpha(\mathrm{eIF} 2 \alpha)$ to attenuate the rate of general translation initiation and prevent further protein synthesis (DuRose et al., 2009). Our focus on GRP78, because GRP78 expression is significantly higher in colon cancer than in colon adenoma and normal tissues (Langer et al., 2008).

\subsubsection{Nuclear factor erythroid-1 and 2-related factor (NFE2L1 or Nrf2)}

NFE2-related factor 1 (NFE2L1), a transcription factor in the cap "n" collar (CNC) basic leucine zipper family, regulates expression of cytoprotective genes. Nuclear factor 
erythroid-derived 2-related factor 1 (NFE2L1) is a member of the CNC subfamily of basicleucine zipper transcription factors (Chan et al., 1998). CNC factors form heterodimers with small-Maf-proteins and regulate transcriptional activation through the antioxidant response element (ARE) located at the promoter region of various antioxidant genes have four NFE2L genes, namely, NFE2L1, NFE2L2, NFE2L3, and nuclear factor erythroid 2 (NF-E2), implying that a diversification of the protein family occurred during vertebrate evolution. The function of Nrf proteins are to regulate the work of antioxidant proteins that can help protect against oxidative damage and responsible for the expression of certain Phase I and Phase II detoxification enzymes (Motohashi et al., 2002). Many studies revealed that NFE2L1, NFE2L2, and NFE2L3 contribute to proteasome subunit gene expression with varying significance (Ohtsuji, at al., 2008). NFE2L1 and NFE2L2 bind to enhancer element known as the antioxidant response element (ARE; consensus sequence: TGCTCAGTCAT) (Dhakshinamoorthy et al., 2005). NFE2L1 and NFE2L2 are expressed across almost all tissues. Although NFE2L2 was initially proposed to increase the expression of proteasome subunits, it was recently demonstrated that NFE2L1 plays a pivotal role in the upregulation of proteasome gene expression and de novo proteasome synthesis in response to proteasome inhibition (Hamazaki and Murata, 2020). NFE2L1 has four isoforms, namely, NFE2L1 $\alpha$, NFE2L1 $\beta$, and NFE2L1 $\gamma$, and NFE2L1 $\delta$; however, it is unknown how each NFE2L1 isoform contributes to regulate the expression of cytoprotective genes against various types of physiopathological stresses (Ohtsuji, at al., 2008). We investigated NFE2L1 and NFE2L2 in relation to WNT signaling during stress conditions and NFE2L1 and NFE2L2 were both activated when cell overexpressed WNT proteins. 


\subsection{Hypothesis and objectives}

WNT signaling pathways plays essential roles during embryonic development and tissue homeostasis to control stem cell renewal, cell proliferation, differentiation, polarity, and migration. Cancer stem cells in many organs and tissues have been linked to the WNT signaling pathway and, in particular, WNT/ $\beta$-catenin signaling has been linked to gastrointestinal tract cancers including colon cancer growth and progression.

Most of the studies on WNT signalling are focused on the downstream of the pathway, indicating the promise of therapeutic targets for colon cancer. While many studies focus on the downstream canonical and non-canonical pathways activated by WNT proteins, few examine the upstream production and processing of WNTs, in response to tumor microenvironment stresses (Fig. 1.13). Examinging WNTs upstream secreation will potentially aid of our understanding of the initiation of colon cancer.

The focus of this thesis is to build upon our current knowledge of the WNT signaling pathway and its regulation in response to hypoxia, endoplasmic retriculum, and oxidative stress. Several studies have reported crosstalk between hypoxia and WNT signaling in the modulation of cancer malignancy and metastasis.

\section{Hypotheses}

$>$ I hypothesized hypoxia, oxidative stress, ER stress, and environmental toxins will alter PORCN and WLS expression. PORCN and WLS will response to these treatments by modifying the WNT signaling during secretion and ultimately change the WNT ligand bding to the receptors. 
I hypothesized that stressors which include hypoxia, ER, oxidative and cadmium will impact PORCN and WLS by activating different transcription factors.

$>$ Transcription factors are commonly deregulated in the pathogenesis of human cancer and are an essential category of cancer cell dependencies.

Transcription factors which are commonly activated in response to stress and implicated in many cancers including colon are GRP78, HIF1 $\alpha$, NFE2L1 and NFE2L2.

I hypothesize that hypoxia, oxidative stresses, ER stress, and environmental toxins will activate GRP78, HIF1 $\alpha$, MT1, NFE2L1, and NFE2L2.

$>$ Environmental toxins such cadmium could potentially be altering WNT signaling due to hypoxia, oxidative stress, ER stress, and environmental toxins.

Cadmium will alter gene and protein expression of WNT secretory components and WNT proteins.

\section{Objectives}

$>$ My first objective in this thesis was to understand the role of stressor on PORCN and WLS in WNT signaling in HCT116 and HIEC-6.

$>$ To understand the implication of PORCN and WLS on the expression of WNT secretion, we knockdown the PORCN and WLS in HCT116 after hypoxia treatment.

$>$ We investigated the implication of inhibiting important post translation modification of palmitoyleation and glycosylation on PORC, WLS, and WNT3A. 
$>$ We determined if GRP78, HIF-1 $\alpha$, NFE2L1, and NFE2L2 are major regulators of PORCN, WLS and WNT3A expression.

$>\mathrm{I}$ was also interested in learning if WNT can be activated after exposure of environmental contaminants such as cadmium chloride. 


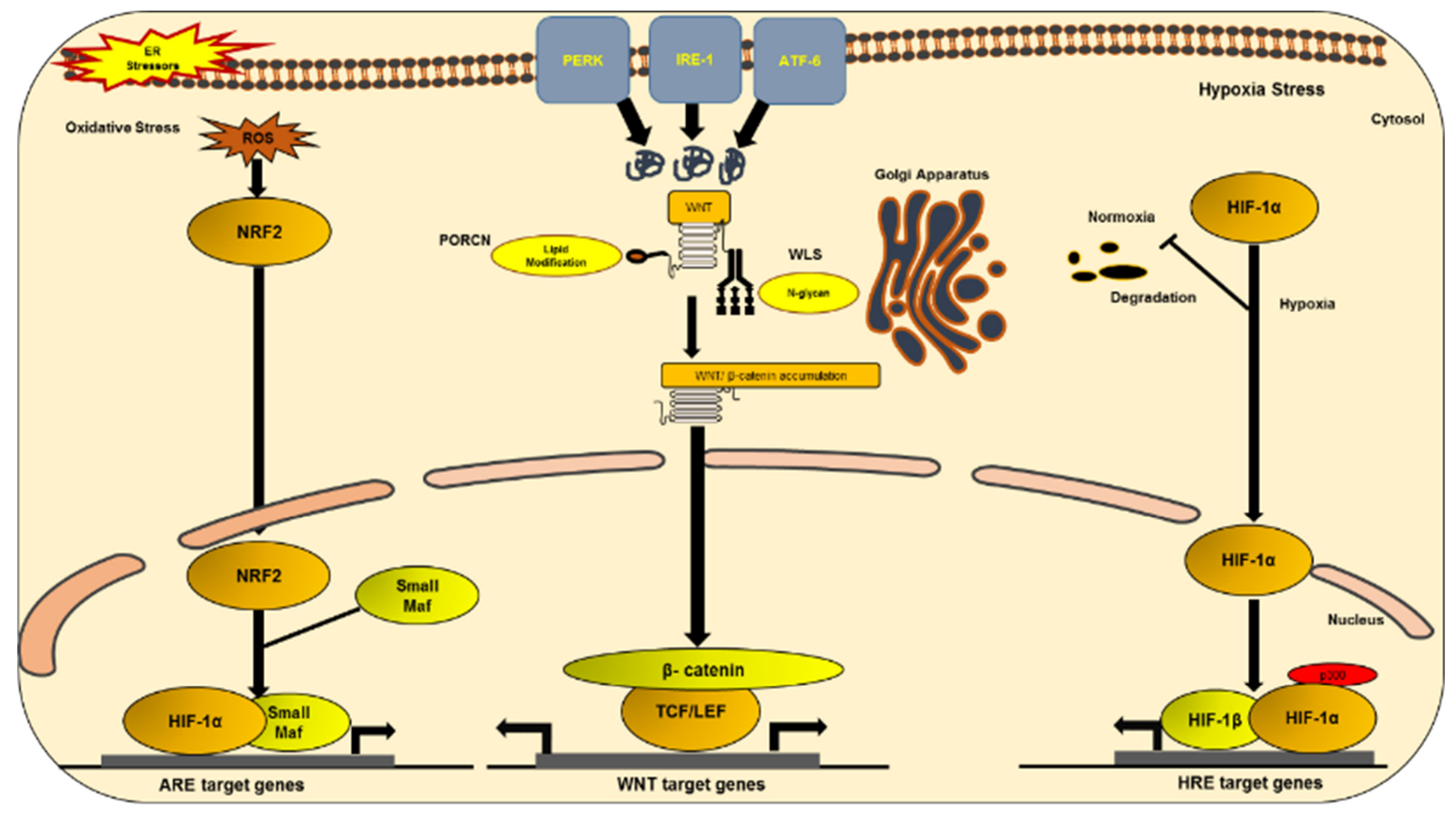


Figure 1.13: Schematic diagram of potential of WNT pathways linked to hypoxia, endoplasmic reticulum (ER) and oxidative stressors which controls and regulates WNT secretion. In the secreting cells the WNT ligand is translated into the ER where it is bound and modified with lipids by the membrane-bound-O-acyltransferase PORCN. The lipidated WNT is transported by a yet unknown mechanism to the Golgi. The transmembrane protein WLS binds to WNT in the Golgi and escorts it to the plasma membrane. From there WNTs are eventually released into the extracellular space. In the WNT-expressing cells WLS is saved from lysosomal degradation by binding to the retromer complex and is retrieved to the trans-Golgi for additional rounds of WNT secretion. This leads to cytoplasmic stabilisation and subsequent nuclear translocation of $\beta$-catenin. In the nucleus $\beta$-catenin associates with transcriptional coactivators and target genes are transcribed ER stress and consequent unfolded protein response (UPR) is important during stress conditions. During ER stress essential proteins released from IRE1 $\alpha$, PERK and ATF6 sensors allowing their dimerization/oligomerization export to the Golgi apparatus. PERK activation leads to phosphorylation of NRF2, HIF1 $\alpha$ and eIF2 $\alpha$ and genes involved in antioxidant response, protein folding, and apoptosis. Hypoxia induces the activation of HIF proteins and activating WNT signaling (Boso et al., 2019). 


\section{Chapter 2: Materials and Methods}

\subsection{Cell culture}

Human embryonic kidney cells that express a mutant version of the SV40 large T antigen (HEK293T) and the human colon cancer adenocarcinoma cell line (HCT116) and human intestinal epithelium cells (HIEC-6) were obtained from the American Type Culture Collection (Manassas, Virginia, USA). Human intestinal Epithelial Cell-6 was donated from Dr. Benoit Yannick (University of Ottawa, Ontario). HEK293T cells were propagated and maintained in Dulbecco's Modified Eagle Medium (DMEM), (Gibco BRL, Rockville, Maryland, USA) and HCT116 were maintained in McCoy's (GE Healthcare Life Sciences, Mississauga, Ontario, Canada) medium. Both medias were supplemented with $5 \%$ fetal bovine serum and 5\% newborn calf serum. HIEC-6 cells were maintained in OptiMEM and supplemented with 5\% Wisent Premium FBS, 20mM HEPES and10 mM Glutamax (Gibco BRL, Rockville, Maryland, USA) and Epidermal Growth Factor (EGF 10 ng/mL). Cells were incubated in $5 \% \mathrm{CO}_{2}$ at $37^{\circ} \mathrm{C}$ and $95 \%$ humidity. The cells reached $90 \%$ confluency before splitting using $1 \mathrm{X}$ trypsin in $1 \mathrm{X}$ phosphate buffer saline (PBS). Cells were counted using a hemocytometer and $1.4 \times 10^{6}$ cells were placed in $10 \mathrm{~cm}$ diameter tissue culture plates for treatment.

\subsection{Assessment of total cell viability}

Cell viability was assessed with the colorimetric MTT metabolic activity assay. Briefly, $20 \mathrm{~mL}$ of a $5 \mathrm{mg} / \mathrm{mL}$ MTT (3-[4,5-di-methylthiazol-2-yl]-2,5- diphenyltetrazolium bromide, Sigma-Aldrich). Cells were treated for 18 to 24 hours with $10 \mu \mathrm{g} / \mathrm{mL}$ of tunicamycin (Tuni), 1.5 and $3.0 \mathrm{nM}$ thapsigargin (Thap), 5 and $10 \mathrm{mM}$ antimycin A (AA), 
3 and $6 \mathrm{mM}$ dithiothreitol (DTT) and the $10 \mu \mathrm{M}$ proteasome inhibitor MG132 (MG132)

for 3.45 hours. In another experiment, cells were exposed to different concentrations of $\mathrm{CdCl}_{2}$ for $24 \mathrm{~h} \mathrm{HCT} 116$ and HIEC-6 cells were cultured in medium as described above, and $180 \mu \mathrm{L}$ aliquots cell suspension $\left(5 \times 10^{5} / \mathrm{mL}\right)$ were pipetted and placed 96 -well polystyrene tissue culture plates, followed by the addition of $20 \mu \mathrm{L}$ aliquots of stock solutions to makeup six replicates of final cadmium chloride concentrations of $0,2.5,5,7.5,10$ and 12.5 $\mu \mathrm{M}$. Control cells received $20 \mu \mathrm{L}$ of distilled water. After chemical treatment, HCT116 and HIEC- 6 cells were incubated for $28 \mathrm{~h}$ in a humidified $5 \% \mathrm{CO}_{2}$ incubator at $37^{\circ} \mathrm{C}$. MTT solution was added to HCT116 and HIEC-6 cells cultured in a 96-well plate at a density of 10,000 cells per well. Following $1 \mathrm{~h}$ incubation, the MTT medium was removed and the insoluble formazan salt was solubilized with $100 \mathrm{~mL}$ of DMSO. Absorbance of converted dye was measured at $570 \mathrm{~nm}$ with a background subtraction at $620 \mathrm{~nm}$ using a spectrophotometer. All the data were calculated by subtraction the DMSO absroance reading from the converted absorbance of the treated samples.

\subsection{Chemicals}

Cadmium chloride $\left(\mathrm{CdCl}_{2}\right)$, Tunicamycin (Tuni), thapsigargin (Thap), antimycin A (AA), dithiothreitol (DTT), deferrioxamine (DFO), 2-bromo-hexadecanoic acid (2BHDA) 3-(4,5 dimethylthiazol-2-yl)-2,5-diphenyltetrazolium bromide (MTT) and the proteasome inhibitor MG132 were purchased from Sigma-Aldrich (Oakville, Ontario, Canada). 3-[4(2-chloro-5-fluorophenoxy)-1-piperidinyl]-6-(5-methyl-1,3,4-oxadiazol-2-yl)-pyridazine (SCD1 inhibitor) Dimethyloxalylglycine (DMOG) were purchased from the Cayman 
Chemical Company (Ann Arbor, Michigan, USA). Oligofectamine was purchased from Invitrogen/ThermoFisher Scientific (Mississauga, Ontario, Canada).

\subsection{Construction of plasmids and gene cloning}

Gene-specific primers (Table 1) with restriction enzyme adaptors were designed (PrimerQuest Tool; Integrated DNA Technologies, Coralville, Iowa, USA) to carry out reverse transcriptase-polymerase chain reaction (RT-PCR) amplification using iProof ${ }^{\mathrm{TM}}$ High-Fidelity DNA Polymerase (Bio-Rad Laboratories, Mississauga, Ontario, Canada). Reactions were run with annealing temperatures ranging from $55^{\circ} \mathrm{C}$ to $65^{\circ} \mathrm{C}$, depending upon the primer set used, for 1 hour. PCR products were run on a $1 \%$ agarose gel and the bands were excised from the gel and weighed for gel removal. Glassmilk was used to purify the PCR products of PORCN and WLS genes from the agarose gel. Samples were suspended using 3 volumes of $6 \mathrm{M}$ sodium iodide and washed by NEW Wash solution (100 $\mathrm{mM} \mathrm{NaCl}, 1 \mathrm{mM}$ EDTA, $10 \mathrm{mM}$ Tris- $\mathrm{HCl} \mathrm{pH} 7.5$ and 50\% ethanol). The PCR products were digested with NdeI and EcoRI-HF and run on a $1 \%$ agarose gel for verification. Digested DNA fragments of PORCN (1,395 b.p.) and WLS (1,635 b.p.) were then cloned into a modified version of the pCR3.1 plasmid which places the FLAG tag at the Nterminus of the gene. Fragments were ligated using T4 DNA ligase (New England Biolabs Ltd., Whitby, Ontario, Canada), the ligation was designed at a molar ratio of 1:3 vector to insert. All plasmids were transformed in DH5 $\alpha$ (Invitrogen/ThermoFisher Scientific, Mississauga, Ontario, Canada). Constructed plasmids were analyzed and sequenced using the T7 promoter universal primer and the Bovine Growth Hormone reverse universal primer (Table 1) (Bio Basic Inc., Markham, Ontario, Canada). 


\subsection{Cellular reactive oxygen species (ROS with DCFDA)}

Formation of ROS was evaluated using the live cell fluorescent ROS detection microscopy. ROS generation was analyzed after staining the cells with dichlorofluorescein-diacetate (DCF-DA) as a fluorescent probe. The DCF fluorescence intensity directly is proportional to the amount of peroxides produced by the cells. Briefly, the cells were incubated with different concentrations of $\mathrm{CdCl}_{2}$ at $0,2.5,5,10 \mu \mathrm{M}$. After exposure to $\mathrm{CdCl}_{2}$, cells were loaded with dichlorofluorescin diacetate $(20 \mathrm{mM})$ in $1 \mathrm{X}$ PBS at $37 \mathrm{C}$ for $30 \mathrm{~min}$ in the dark. The cells were then washed with $1 \mathrm{X}$ PBS and fluorescence caused by DCF in each well was observed through a fluorescent microscope (EVOS M5000 Imaging system, ThermoFisher Scientific, Mississauga, Ontario, Canada).

\subsection{Transfections}

The two constructed plasmids, PORCN and $W L S$ in pCR3.1, were transiently transfected into HEK293T cells. The cells were grown in $10 \mathrm{~cm}$ diameter tissue culture plates to $80 \%$ confluency and transiently transfected with $20 \mu \mathrm{g}$ of plasmids via polyethylenimine (PEI; Sigma Aldrich, Oakville, Ontario, Canada). Cell pellets were stored at $-20^{\circ} \mathrm{C}$ for protein extraction. HCT116 cells were seeded in six-well plates at a density of $2 \times 10^{5}$ cells per well and allowed to grow to $30 \sim 50 \%$ confluency. HCT116 were then transfected using Oligofectamine (Invitrogen, Carlsbad, California, USA), according to the manufacturer's instructions. Briefly, for each well to be transfected, $15 \mu \mathrm{L}$ Oligofectamine and $15 \mu \mathrm{L}$ of $100 \mathrm{nM}$ siRNA for PORCN or WLS or WNT3A of expression were mixed separately with $150 \mu \mathrm{L}$ Opti-MEM (Invitrogen, Carlsbad, 
California, USA) each. PORCN, WLS and WNT3A plasmids were prepared using midiprep (Promega, Madison, USA). The two solutions were combined, mixed gently, and incubated at room temperature for 15 minutes. The two solutions were combined, mixed gently, and incubated at room temperature for 15 minutes. The mixture was then added to the serum-free media in each well. Cells were incubated in this mix at $37^{\circ} \mathrm{C}$ for $72 \mathrm{hrs}$. Cell viability was assessed, following treatments, by MTT assay to assess the toxicity of each treatment. Cells were treated for 18 to 24 hours with $10 \mu \mathrm{g} / \mathrm{mL}$ of tunicamycin (Tuni; HEK293T and HCT116), 1.5 and 3.0 nM thapsigargin (Thap; for HEK293T and HCT116 respectively), 5 and $10 \mathrm{mM}$ antimycin A (AA; for HEK-293T and HCT116 respectively), 3 and $6 \mathrm{mM}$ dithiothreitol (DTT; HEK293T and HCT116 respectively) and the $10 \mu \mathrm{M}$ proteasome inhibitor MG132 (MG132; HEK293T and HCT116) for 3.45 hours. Cells were scraped in $1 \mathrm{X}$ PBS, harvested, and centrifuged at 1,000 X g for 5 minutes. Samples were treated with hypoxia for $24 \mathrm{hrs}$ after $48 \mathrm{hrs}$ of transient transfection ( $72 \mathrm{hrs}$ total). Samples were harvested for protein extractions as mentioned previously.

\subsection{Hypoxia and hypoxia mimetic treatments}

Cells were treated in the hypoxia chamber for 24 hours at $1 \% \mathrm{O}_{2}$ and $5 \% \mathrm{CO}_{2}$ at $37^{\circ} \mathrm{C}$ and $95 \%$ humidity. Cells were harvested within 5 minutes by scraping into $1 \mathrm{X}$ PBS, which was bubbled with nitrogen for 5 minutes. DMOG $(1 \mathrm{mM})$ and DFO $(100 \mu \mathrm{M})$ were used to inhibit oxygen sensor effects in cells for 24 hours. Cells were harvested in 1 X PBS and pellets were stored at $-20^{\circ} \mathrm{C}$. 


\subsection{Gene expression by quantitative polymerase chain reaction (qPCR)}

Total RNA was isolated from cells using RNeasy Mini Qiagen Kit (QIAGEN, Hilden, Germany) and reverse transcribed to cDNA using $1 \mu \mathrm{g}$ of total RNA with iScript cDNA Synthesis Kit and a Bio-Rad iCycler thermocycler (Bio-Rad Laboratories, Mississauga, Ontario, Canada). Glyceraldehyde-3-phosphate-dehydrogenase (GAPDH) served as the "housekeeping" gene. Gene-specific primers for different genes, were designed using PrimerDesigner (Integrated DNA Technologies, Coralville, Iowa, USA) and the specificity of the primers were determined by a National Center for Biotechnology Information (NCBI) Basic Local Alignment Search Tool (BLAST) search of the human genome (Table 2). Primers and diluted cDNA samples were prepared with SsoAdvanced Universal Syber Green Supermix (Bio-Rad Laboratories, Mississauga, Ontario, Canada) for qPCR. For the thermal cycle reaction, the CFX System, Version 3.1 (Bio-Rad Laboratories, Mississauga, Ontario, Canada) was used and the melting temperature was set at $95^{\circ} \mathrm{C}$ for 2 to $3 \mathrm{~min}$, then 40 cycles of $95^{\circ} \mathrm{C}$ for $15 \mathrm{sec}$ and at $60^{\circ} \mathrm{C}$ for $5 \mathrm{sec}$. The relative amount for each transcript was calculated by a standard curve of cycle thresholds for serial dilutions of cDNA samples and normalized to GAPDH (Table 2). The polymerase chain reaction (PCR) was performed in triplicate for each sample, after which all experiments were repeated four times.

\subsection{Membrane-bound protein extraction}

Frozen pellets of cells were dissolved in $8 \mathrm{M}$ urea and incubated for 1 hour at $4^{\circ} \mathrm{C}$. To obtain the membrane-bound fraction, samples underwent ultracentrifugation at 100,000 $\mathrm{X} g$ for 1 hour at $4^{\circ} \mathrm{C}$. The supernatants of the samples were isolated and frozen. The 
amount of protein was quantified using the Pierce BCA Protein Assay Kit (ThermoFisher Scientific, Mississauga, Ontario, Canada).

\subsection{Gel electrophoresis and immunoblotting analysis}

Extracted proteins (30-50 $\square \mathrm{g}$ ) were run on a $10 \%$ SDS-polyacrylamide gel (BioRad Laboratories, Mississauga, Ontario, Canada). Gels were run at $90 \mathrm{~V}$ for 2.5 hours. Following electrophoresis, proteins were electroblotted onto a $0.45 \mu \mathrm{M}$ pore size polyvinylidene difluoride (PVDF; Bio-Rad Laboratories, Mississauga, Ontario, Canada) membrane soaked in $100 \%$ methanol by wet transfer with a transfer buffer solution. The membrane was washed five times for 5 minutes, each with 3-5 mL of Tris-buffered saline. For PORCN and WLS, the blots were then blocked with 5\% w/v skim milk dissolved in 1 $\mathrm{X}$ PBS and $2 \%$ sucrose for 1 hour. Blots were incubated overnight at $4{ }^{\circ} \mathrm{C}$ with primary antibodies to PORCN and WLS (Novus Biologicals, Oakville, Ontario, Canada) diluted in $1 \mathrm{X}$ PBS and $2 \%$ sucrose $(1: 500 \mathrm{v}: \mathrm{v})$. Membranes were then washed 5 times for 5 min with $1 \mathrm{X}$ PBS and $2 \%$ sucrose, and incubated with goat anti-rabbit IgG-HRP (Bioshop Canada, Burlington, Ontario, Canada) for 1 hour in 1:5,000 v:v 1 X PBS and $2 \%$ sucrose. For $\beta$-cateinin, blots were then blocked with $5 \% \mathrm{w} / \mathrm{v}$ skim milk dissolved in $1 \mathrm{X}$ TBST for 1 hour at room temperature. Blots were incubated for 1 hour at room with primary antibodies to $\beta$-catenin (12F7) (Novus Biologicals, Oakville, Ontario, Canada) diluted in $5 \% \mathrm{w} / \mathrm{v}$ skim milk in TBST $(1: 1,000 \mathrm{v}: \mathrm{v})$ with a small amount of sodium azide added. Membranes were then washed 3 times for 30 min with 1 X TBST, and incubated with appropriate secondary antibody conjugated with horseradish peroxidase (goat anti-mouse 
IgG-HRP; Dako; Denmark) for 1 hour; antibody was diluted 1:5,000 v:v in 5\% w/v skim milk dissolved in $1 \mathrm{X}$ TBST for 1 hour at room temperature.

\subsection{Promoter analysis and electrophoretic mobility shift assay}

In silico analysis of potential hypoxia response elements (HREs), antioxidant response elements (AREs), NF- $\mathrm{kB}$ binding sites was undertaken using the ExPASy Bioinformatics Resources Portal. A $10 \mathrm{~Kb}$ region in the promoters of the PORCN and $W L S$ genes were searched for core HREs (sequence ACGTGCGCG), AREs (sequences ATtAAGCATGC, GCTAAGCATGt, GAATGCTTCTA and AGCTAGCATTT) and NF- $\mathrm{kB}$ binding sites (sequence TTGGTGTTTCC) (Fig. 1). The PORCN promoter contained one putative NF-kB-binding site (-1,652 to -1,662 b.p.), two putative AREs (1,455 to $-1,465$ b.p. and -533 to -543 b.p.) and one putative HRE (-295 to -303 b.p.). Of these, the ARE at -1.455 did not show binding in EMSAs and was abandoned (data not shown). The WLS promoter contained two putative AREs ( -299 to -309 and -815 to -825 b.p.) and one putative HRE (-648 to -656 b.p.). Of these, they ARE at -299 did not show binding in EMSAs and was abandoned (data not shown). No potential NF-KB binding sites were identified in the WLS promoter region. 


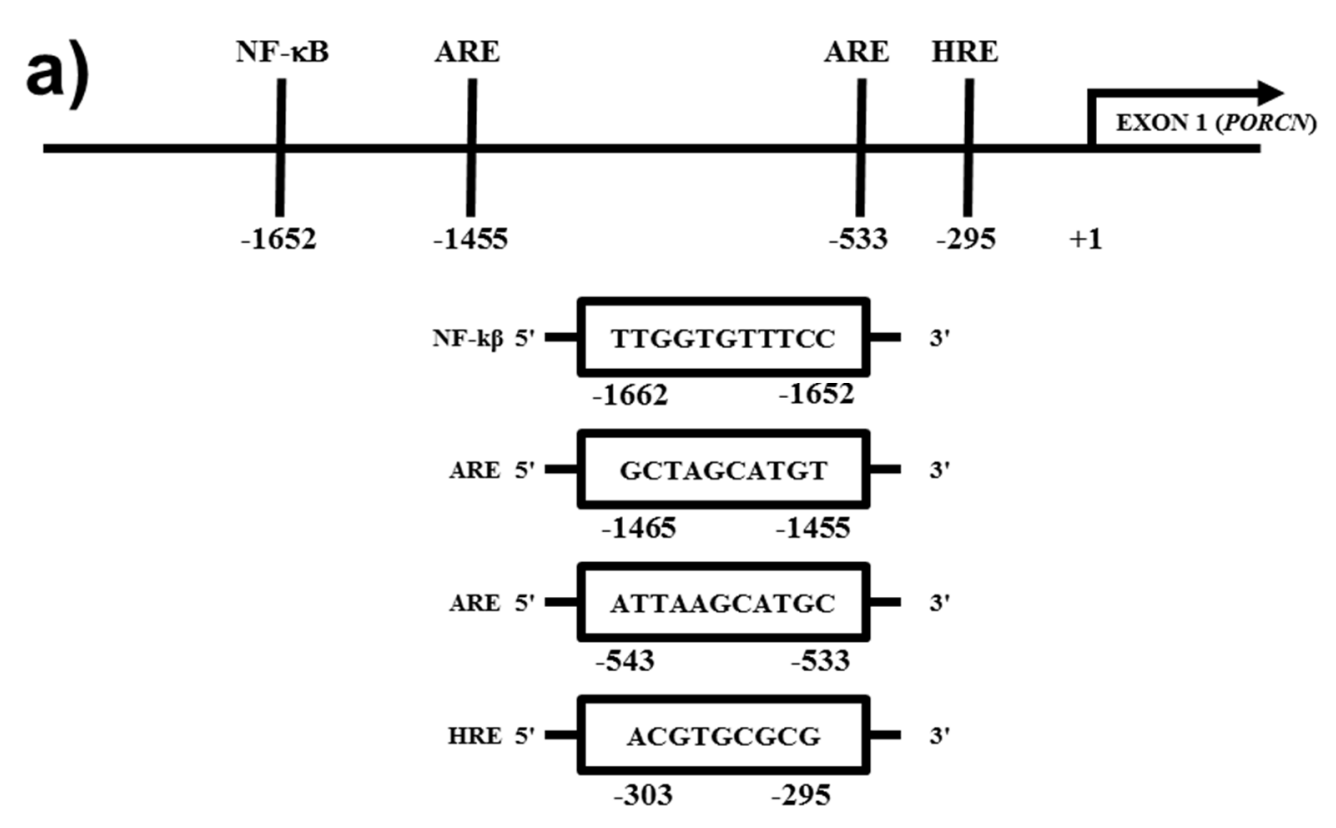

b)
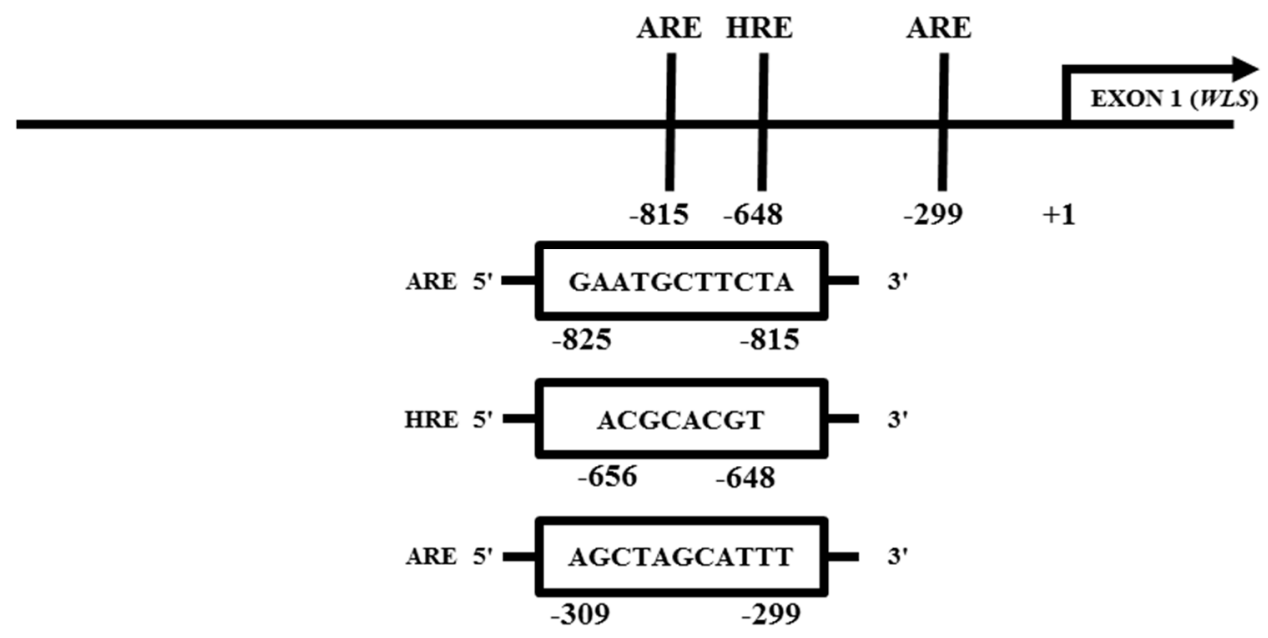

Figure 2.1: Promoter analysis of (a) PORCN and (b) $W L S$ genes. Analysis of the PORCN and $W L S$ promoters was performed using the ExPASy Bioinformatics Resources Portal. Antioxidant Response Elements (AREs), Hypoxia Response Elements (HREs) and NF- $\mathrm{BB}-$ binding sites were identified and further tested with Electrophoretic Mobility Shift Assays (EMSAs) using promoter-specific sequences. 


\subsubsection{Preparation of whole cell extracts}

HEK293T cells were grown in $6 \mathrm{~cm}$ diameter tissue culture plates, transiently transfected with PEI and treated as previously described. Treated cells were harvested using $1 \mathrm{X}$ PBS and lysed using lysis buffer (20 mM HEPES (pH 7.9), $420 \mathrm{mM} \mathrm{NaCl}, 1.5$ $\mathrm{mM} \mathrm{MgCl} 2,0.2 \mathrm{mM}$ EDTA, $25 \%$ glycerol, $500 \mathrm{mM}$ PMSF and $500 \mathrm{mM}$ DTT). Lysed samples were incubated on ice for $5 \mathrm{~min}$. Cells pellet were kept cool and were centrifuged at $12,000 \mathrm{Xg}$ for $15 \mathrm{~min}$ at $4^{\circ} \mathrm{C}$. Supernatants were removed and placed into new precooled microcentrifuge tubes. Protein quantity was determined using the Pierce BCA Protein Assay Kit (ThermoFisher Scientific, Mississauga, Ontario, Canada).

\subsubsection{Probe synthesis}

Commercially synthesized oligonucleotide strands were 5'-biotin end-labelled DNA designed by Integrated DNA Technologies (IDT) (Coralville, Iowa, USA). Targets were annealed in sterile water by mixing an equal number of moles of each strand together, boiled for $1 \mathrm{~min}$. and then slowly cooled down to room temperature.

\subsubsection{Binding reactions}

Gelshift Chemiluminescent EMSA Kit (Active Motif, Carlsbad, California, USA) components were used in the reaction mixture (10 X binding buffer, poly (dI-dC) and $5 \mathrm{X}$ loading buffer). Protein $(7.5-10 \mu \mathrm{g})$ from whole cell extracts were used in the mixture and incubated at room temperature for 20 mins. Samples were then loaded into a pre-run $5 \%$ native polyacrylamide gel $(15.126 \mathrm{~mL}$ total), containing BioRad $30 \%$ Acrylamide/Bisacrylamide Mix (29:1; $2.500 \mathrm{~mL}), 0.5$ X TBE (10 mM Tris base, $10 \mathrm{mM}$ 
boric acid, $0.3 \mathrm{mM}$ EDTA; $0.750 \mathrm{~mL})$, freshly prepared $30 \%$ ammonium persulfate $(0.120$ $\mathrm{mL})$ and TEMED $(0.006 \mathrm{~mL})$ for a $10 \%$ separation gel. Native polyacrylamide gels were pre-run for $1 \mathrm{hr}$ at $100 \mathrm{~V}$ in $0.5 \mathrm{X}$ TBE buffer. Whatman $0.2 \mu \mathrm{m}$ nylon blotting membranes (NytranN; Sigma-Aldrich, Oakville, Ontario, Canada) were soaked in $0.5 \mathrm{X}$ TBE buffer. Proteins were transferred from gel to membrane at $380 \mathrm{~mA}$ and $100 \mathrm{~V}$ for $55 \mathrm{~min}$ using a Bio-Rad Mini-PROTEAN Tetra Cell system (Bio-Rad Laboratories, Mississauga, Ontario, Canada). Membranes were placed to air dry for $1 \mathrm{~min}$ at room temperature and placed inside a commercial UV-light cross-linker and cross-linked for 60 seconds with $\mathrm{mJ} / \mathrm{cm}^{2}$. Membranes were blocked overnight using a blocking buffer (1 X PBS and 2\% sucrose and $0.5 \%$ of skim milk) and washed 5 times with $4 \mathrm{X}$ washing buffer (1 X PBS and $2 \%$ sucrose). Substrate equilibration buffer was added to the membranes for 5 mins. Chemiluminescent working solution was added to the membranes and blots were imaged with a chemidoc system equipped CCD Camera (Fusion Fx Viber, Lourmat, France).

\subsection{Knockdown of PORCN and WLS using siRNA}

The PORCN and WLS gene sequences from GenBank (Accession number NM_203475.3221 and NM_024911.7) and a universal negative control DsiRNA (IDT, Coralville, Iowa, USA) were used to find target sequences for RNA interference. Small interfering RNAs (siRNAs) targeting the PORCN and WLS genes were designed and synthesized by IDT (Coralville, Iowa, USA). The sequence for PORCN and WLS are shown in Table 1. These sequences were checked for nonhomology to any human gene using BLAST. For RNA interference (RNAi), HCT116 cells were seeded in six-well plates at a density of $2 \times 10^{5}$ cells per well and allowed to grow to $30 \sim 50 \%$ confluency. Cells were 
then transfected using Oligofectamine (Invitrogen, Carlsbad, California, USA), according to the manufacturer's instructions. Briefly, for each well to be transfected, $15 \mu \mathrm{L}$ Oligofectamine and $15 \mu \mathrm{L}$ of $100 \mathrm{nM}$ siRNA for PORCN or WLS of expression were mixed separately with $150 \mu \mathrm{L}$ Opti-MEM (Invitrogen, Carlsbad, California, USA) each. The two solutions were combined, mixed gently, and incubated at room temperature for 15 minutes. The mixture was then added to the serum-free media in each well. Cells were incubated in this mix at $37^{\circ} \mathrm{C}$ for $72 \mathrm{hrs}$. Samples were treated with hypoxia for $24 \mathrm{hrs}$ after $48 \mathrm{hrs}$ of transient transfection (72 hrs total). Samples were harvested for protein extractions as mentioned previously.

\subsection{Inhibition of WNT post-translation modification in the secretory route}

\subsubsection{PNGase F assay (inhibition of glycosylation)}

HCT116 cells were seeded in six-well plates at a density of $2 \times 10^{5}$ cells per well and allowed to grow to $30 \sim 50 \%$ confluency and treated with hypoxia for $24 \mathrm{hrs.} \mathrm{PNGase}$ F (New England Biolabs, USA) Denaturing conditions were used for the inhibiting glycosylation of PORCN and WNT proteins. $15 \mu \mathrm{g}$ of glycoprotein was mixed with 1ul of $10 \mathrm{X}$ glycoprotein denaturing buffer, $\mathrm{H}_{2} \mathrm{O}$ was added to make up the 10:1 total reaction volume in $1.5 \mathrm{ml}$ Eppendorf tube then heated for 10 minutes at $100^{\circ} \mathrm{C}$. Reaction mixture was cooled down on ice and centrifuge for 10 seconds. $2 \mu \mathrm{L}$ of 10X GlycoBuffer 2 (10X), $2 \mu 1$ 10\% NP-40 and $6 \mu 1 \mathrm{H}_{2} \mathrm{O}$. PNGase $\mathrm{F}(1 \mu \mathrm{L})$ was added and mixed gently and incubated at $37^{\circ} \mathrm{C}$ for 1 hour. Samples were analyzed using western blotting to quantify the protein contents and expression. 


\subsubsection{Inhibition of palimatoylation using 2-bromohexadecanoic acid (2BHDA) and Stearoyl-CoA-Desaturase 1 (SCD1) inhibitor}

HCT116 cells were seeded in six-well plates at a density of $2 \times 10^{5}$ cells per well and allowed to grow to $30 \sim 50 \%$ confluency. Cell viability was assessed, following treatments, by MTT assay to assess the toxicity of each treatment. A $100 \mathrm{mM}$ stock of 2BHDA and SCD1 inhibitor were prepared in DMSO and then diluted 1:1,000 into media for a final concentration of $100 \mu \mathrm{M}$ of $2 \mathrm{BHDA}$ and $10 \mathrm{mM}$ of SCD1 inhibitor. Cells were incubated with or without $100 \mu \mathrm{M}$ 2-bromopalmitate (2BP) for $6 \mathrm{hrs}$ and $10 \mathrm{mM}$ of SCD1 inhibitor for $24 \mathrm{hrs}$. Knockdown samples were also treated with 2BHDA and further placed in either normoxic or hypoxic conditions for 24 hrs. Proteins were quantified using immunoblotting for the disappearance of the modified versions of the proteins. Total RNA was isolated from cells using RNeasy Mini Qiagen Kit (QIAGEN, Hilden, Germany) and reverse transcribed to cDNA using $1 \mu \mathrm{g}$ of total RNA with iScript cDNA Synthesis Kit and a Bio-Rad iCycler thermocycler (Bio-Rad Laboratories, Mississauga, Ontario, Canada). Glyceraldehyde-3-phosphate-dehydrogenase (GAPDH) served as the "housekeeping" gene. Gene-specific primers for NFE2L1 and NFE2L2, HIF1 $\alpha$, GRP78, PORCN and $W L S$, were designed using PrimerDesigner (Integrated DNA Technologies, Coralville, Iowa, USA) and the specificity of the primers were determined by a National Center for Biotechnology Information (NCBI) Basic Local Alignment Search Tool (BLAST) search of the human genome (Table 1). 


\subsection{Statistical analysis}

Gene and protein data was quantified and analyzed using SigmaPlot and statistical analysis was performed using SigmaStat, Version 12.3 (Systat Software, Inc., San Jose, California, USA), respectively. One-way ANalysis OfVAriance (ANOVA) was performed followed by Dunnett's post-hoc test and significant differences were determined with a pvalue of $\leq 0.05$. The following symbols were used to denote statistical significance $* \mathrm{p}<$ $0.05, * * \mathrm{p}<0.01, * * * \mathrm{p}<0.001$ 


\section{Chapter 3: Responses of Porcupine and WNTless proteins to oxidative, hypoxic and endoplasmic reticulum stresses}

Mohamed, R., Kennedy, C., and Willmore, W. G. (2021). Responses of Porcupine and Wntless proteins to oxidative, hypoxic and endoplasmic reticulum stresses. Cellular signalling, 85, 110047.

\subsection{Introduction}

The WNT (Wingless and Int-1) proteins play a role in stem cell development and cell differentiation. Mutations in the WNT proteins lead to the development of various tumours, including colon tumours. Porcupine (PORCN) is a palmitoyltransferase and WNTless (WLS) is a dedicated WNT transport protein that modify and fold the WNT proteins respectively and are involved in their proper secretion and binding to the frizzled (FZD) receptor and the lipoprotein receptor-related protein 5 or 6 (LRP5/6).

While many studies focus on the downstream canonical and non-canonical pathways activated by WNT proteins, few examine the upstream production and processing of WNTs, in response to tumour microenvironment stresses. Porcupine (PORCN) is a member of the membrane-bound O-acyl transferase (MBOAT) family of multipass membrane proteins that catalyze the O-palmitoleation of WNT proteins in the ER. OPalmitoleation involves the attachment of palmitoleic acid, a 16-carbon monounsaturated fatty acid (C16:1), to the target protein. The transmembrane PORCN protein itself is palmitoylated and acylated (Gao and Hannoush, 2014). Once palmitoleated, WNT is passed from the ER to the Golgi complex, where it is glycosylated and transferred to 
secretory vesicles by a dedicated WNT transporter protein Wntless (WLS; also known as GPR177, Evi or Sprinter) (Coombs et al., 2010). WLS is a conserved transmembrane protein that regulates WNT trafficking from the Golgi to the cell membrane (Logan and Nusse, 2004). The gene is highly conserved throughout evolution (Clevers and Nusse, 2012). WLS contains a long $\mathrm{N}$ terminal region, seven or eight transmembrane segments, and is N-linked glycosylated (Logan and Nusse, 2004; Clevers and Nusse, 2012). It is localized throughout the entire WNT secretory pathway, which includes the ER, Golgi, vesicles and plasma membrane (Komiya and Habas, 2008; Belenkaya et al., 2008). The mechanism by which WLS promotes WNT secretion still remains poorly understood.

The present study examined the role and function of PORCN and WLS proteins in the production and modification of WNT3A proteins under the stresses that occur in the tumour microenvironment. The expression of PORCN, WLS and $\beta$-catenin under hypoxia stress, oxidative stress and ER stress conditions, as well as the modification of WNT3A, were investigated. The role of transcription factors that are key players in these stresses (HIF-1, NFE2L, and NF-kB), in PORCN and WLS production was determined. The implications of the activation of these factors on the WNT3A signaling pathway is discussed. 


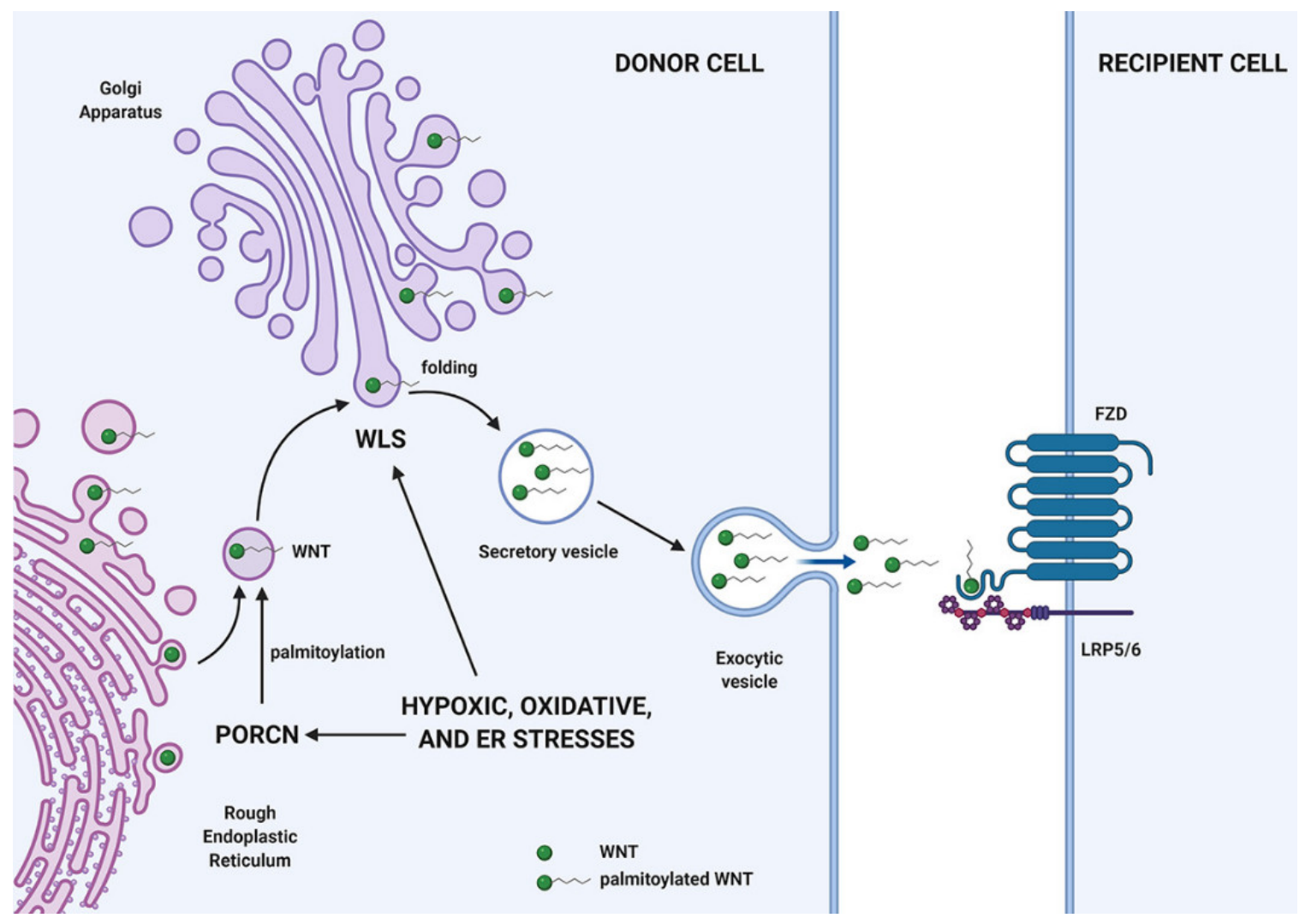

Figure 3.1: The graphical abstract showing how WNT secretory route potentially cross talk with hypoxia, oxidative, and ER stressors. The illustration displays modification of PORCN (palmitoylation) and WLS (glycosylation) could altered as a result of physiological and chemical stresses. Moreover, these changes possibly affect the binding of WNT to the receptors FZ/LPR5/6 (image created by Dr. William Willmore using BioRender). 


\subsection{Results}

\subsubsection{Promoter analysis and electrophoretic mobility shift assay}

In silico analysis of potential hypoxia response elements (HREs), antioxidant response elements (AREs) and NF- $\mathrm{B}$ binding sites was un-dertaken using the ExPASy Bioinformatics Resources Portal. A $10 \mathrm{~Kb}$ region in the promoters of the PORCN and WLS genes were searched for core HREs (sequence ACGTGCGCG), AREs (sequences ATtAAGCATGC, GCTAAGCATGT, GAATGCTTCTA and AGCTAGCATTT) and NF- $\kappa$ B binding sites (sequence TTGGTGTTTCC) (Fig. 2.1). The PORCN promoter

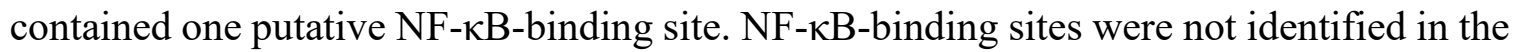
WLS promoter region.

\subsubsection{The effect of hypoxia and hypoxia mimetics condition on the gene expression of PORCN and WLS in HCT116 cells}

HCT116 cells were treated with normoxia $\left(21 \% \mathrm{O}_{2}\right)$, hypoxia $\left(1 \% \mathrm{O}_{2}\right)$ and hypoxic mimetics DMOG $(1 \mathrm{mM})$ and DFO $(100 \mu \mathrm{M})$ for $24 \mathrm{hrs}$. HCT116 cells showed significantly increased gene expression of PORCN with hypoxia, DMOG and DFO treatments and WLS in hypoxia and DMOG treatments (Fig. 3.2a and b). DFO treatment did not result in an increase in WLS gene expression in HCT116 cells (Fig. 3.2b).

\subsubsection{Effect of physicochemical stressors on the gene expression of PORCN and}

\section{WLS on HCT116 cells}

The effects of Tuni, Thap, AA, DTT and MG132 were tested to evaluate their effects on gene expression. HCT116 cells showed significant inductions of PORCN gene 
expression with Thap, AA and MG132 treatments, with the highest expression seen with MG132 treatment (Fig. 3.2c). HCT116 cells showed significant inductions of WLS gene expression with Tuni, Thap, AA, DTT, and MG132 treatments, again with the highest expression seen with MG132 treatment (Fig. 3.2d). Interestingly, both genes showed similar elevations in gene expression between the various treatments. 


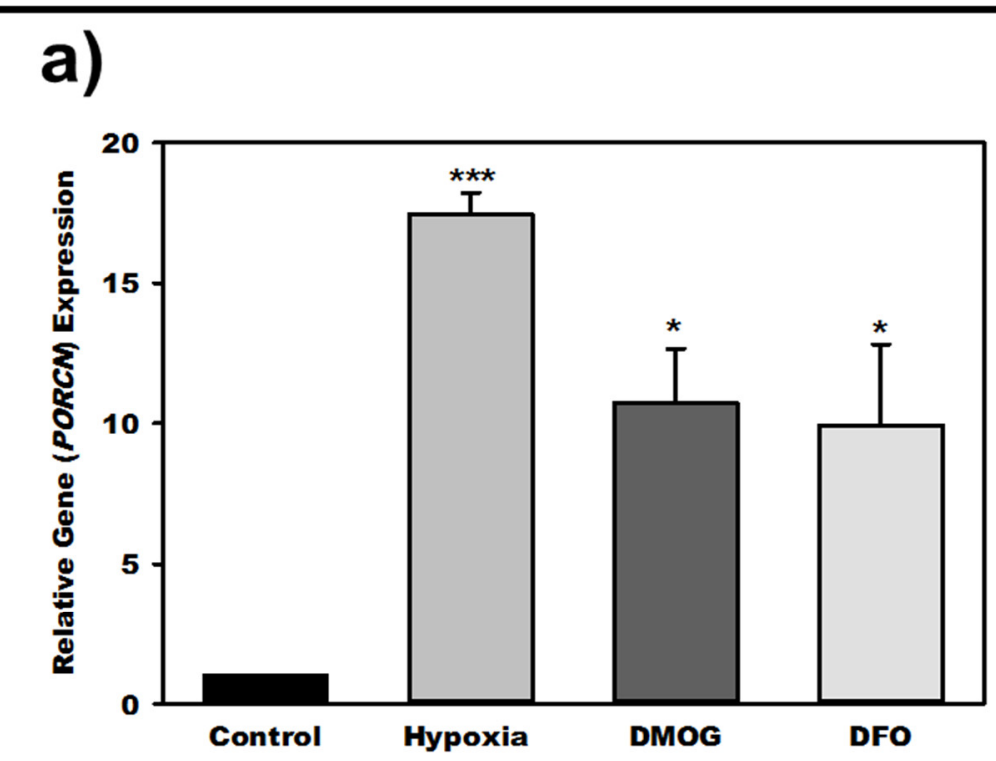

b)

c)
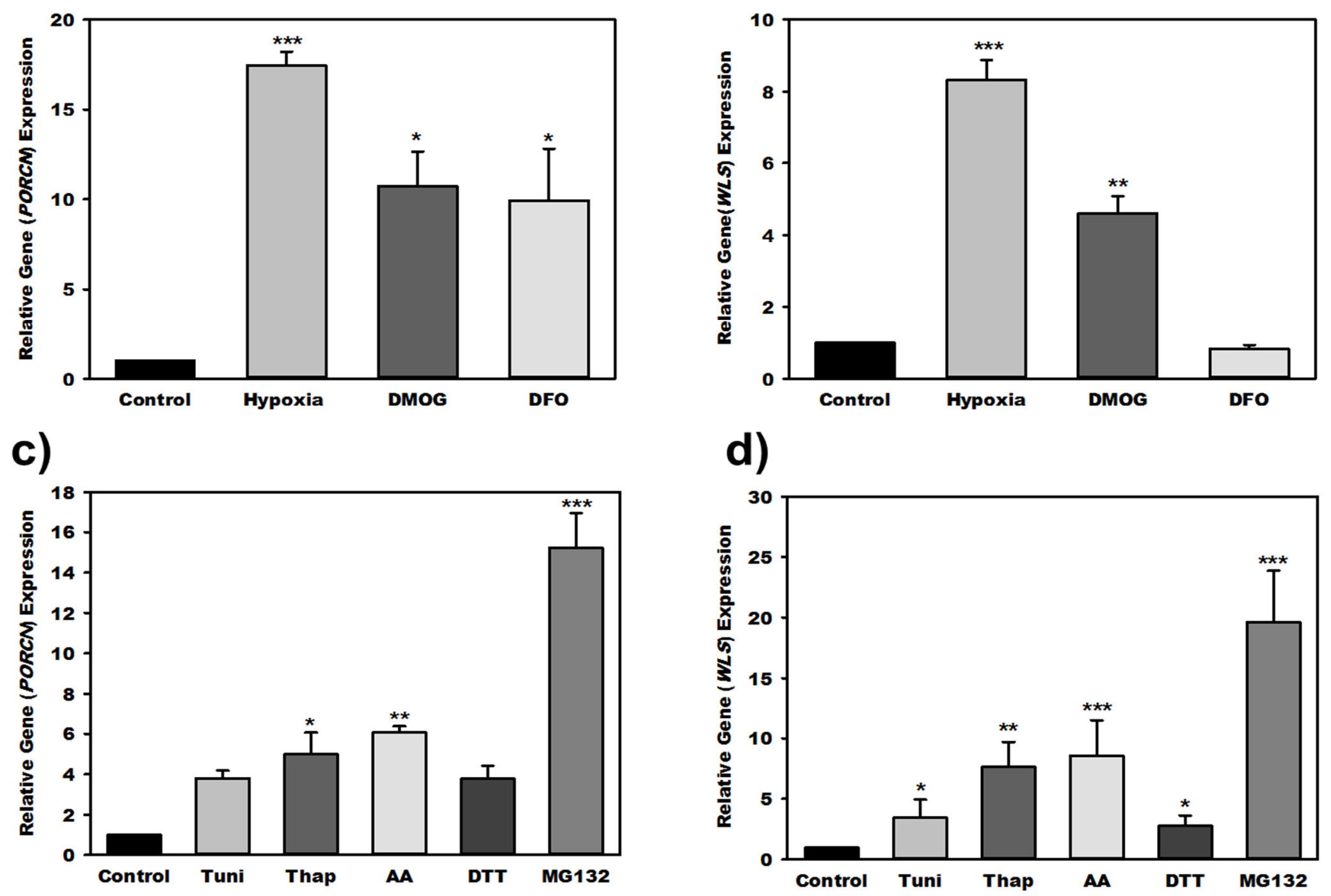

d)

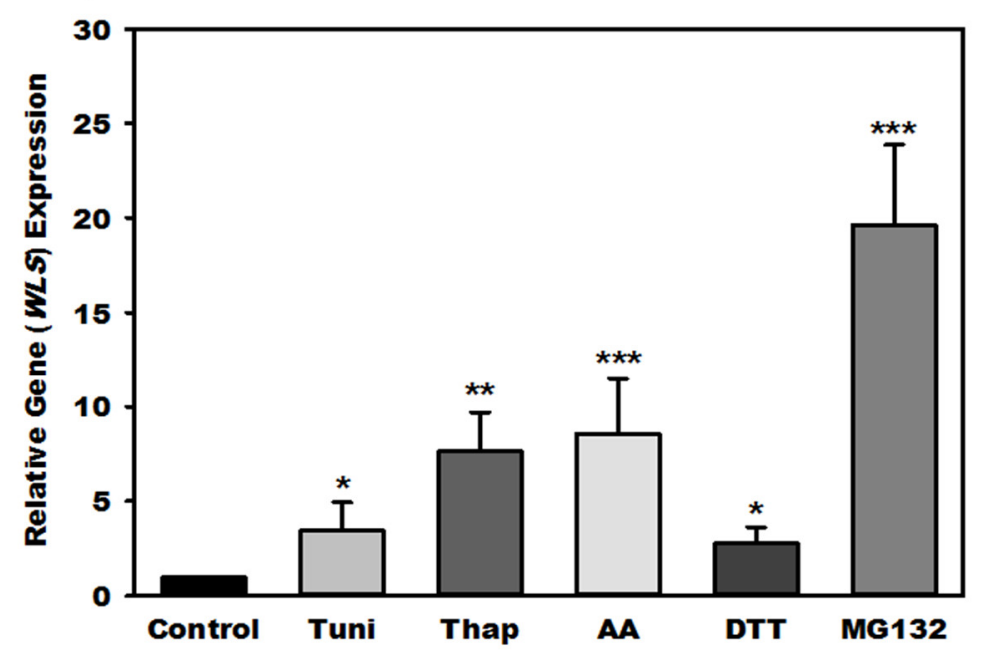


Figure 3.2: The effect of hypoxia and hypoxic mimetics (a and b), ER stressors and oxidative stressors (c and d) on PORCN and WLS gene expression in human colorectal carcinoma (HCT116) cells. HCT116 cells were treated with hypoxia $\left(1 \% \mathrm{O}_{2}\right.$ and $\left.5 \% \mathrm{CO}_{2}\right)$ and hypoxic mimetics (100 $\mu \mathrm{M}$ desferrioxamine (DFO) and $1 \mathrm{mM}$ dimethyloxalylglycine (DMOG), (5 mg/mL tunicamycin (Tuni), $3 \mathrm{nM}$ thapsigargin (Thap), $90 \mathrm{mM}$ antimycin A (AA), $2 \mathrm{M}$ dithiothreitol (DTT) for $24 \mathrm{~h}$ and $5 \mathrm{mg} / \mathrm{mL}$ MG132 for $3.5 \mathrm{~h}$. Cells without treatment were used as controls. Total RNA was isolated from HCT116 cells, converted to cDNA and analyzed by qPCR using gene- specific primers for $a$ and $\mathrm{c}(\mathrm{PORCN})$ and $\mathrm{b}$ and $\mathrm{d}$ (WLS) and target genes. The level of target genes of each mRNA was normalized to the GAPDH mRNA. Data are the means from three independent experiments \pm S.E.M. Data analysis was done using one-way ANOVA followed by Dunnett's test; ${ }^{*} \mathrm{p}<0.05, * * \mathrm{p}<0.01, * * * \mathrm{p}<0.001$. 


\subsubsection{PORCN and WLS response are activated upon exposure to ER stressors}

Cell treatments were utilized to investigate changes in PORCN, WLS and $\beta$-catenin protein expression in HIEC-6 (Panel a) and HCT116 (Panel b) cells (Fig. 3.3), due to hypoxia (hypoxia $(\mathrm{H})$, dimethyloxalylglycine (DMOG) and desferrioxamine (DFO)), ER stress (Tunicamycin (Tuni), Thapsigargin (Thap), dithiolthreitol (DTT) and proteasome inhibition (MG132)) and oxidative stress (antimycin A (AA)). Western blot revealed the presence of PORCN (55 kDa), WLS (65 kDa) and $\beta$-catenin (92 kDa) in HIEC-6 and HCT116 cells, but primarily when induced by treatment. Expression of all three proteins was low in non-treated cells. Treatments with Thap, DTT and MG132 resulted in the highest PORCN expression in HCT116 and treatment with Tuni in HIEC-6 cells (Fig. 3.3, Lanes 3, 5 and 6 respectively). Induction of PORCN protein expression was also seen with MG132 and AA (Fig. 3.3, Lanes 2 and 4), but not to the same extent. WLS protein expression was lower in HCT116 cells, than in HIEC-6 cells, and was most highly induced by DTT (Fig. 3.3, Lane 4). In HCT116 cells, WLS was induced with all treatments, but was highest with Thap, DTT and MG132 (Fig. 3.3, Lanes 3, 5 and 6). Likewise, $\beta$-catenin was induced by all treatments, but was most highly induced by Tuni and MG132 (Fig. 3.3, Lanes 2 and 6). The expression of PORCN and WLS were higher than $\beta$-catenin in both cell lines when treated with ER stressors. The expression of all the proteins PORCN, WLS and $\beta$-catenin was higher in HCT116 than in HIEC-6 cells. 


\subsubsection{Hypoxia and hypoxic mimetics lead to elevated and modified protein expression in PORCN and WLS}

To investigate the role of low oxygen on PORCN and WLS expressions HCT116 and HIEC-6 cells were treated with hypoxia $\left(1 \% \mathrm{O}_{2}\right)$, or the hypoxic mimetics DMOG or DFO, for 24 hrs. Hypoxia and DMOG strongly induced PORCN and, to a lesser extent, WLS compared to controls in both HCT116 and HIEC-6 cells (Fig. 3.3, Lanes 8 and 9). Hypoxia also strongly induced $\beta$-catenin but DMOG did not. DFO induced PORCN, but very strongly induced WLS, in both HCT116 and HIEC-6 cells compared to controls (Fig. 3.3, Lane 10). $\beta$-catenin was not significantly induced by DFO. Overall, HCT116 cells exhibited higher expression of WLS, in all treatments, compared to HIEC-6 cells. The expression of PORCN and WLS were higher than $\beta$-catenin in both cell lines when treated with hypoxia and hypoxia mimetics. The expression of all the proteins PORCN, WLS and $\beta$-catenin was higher in HCT116 than in HIEC-6 cells. 


\section{HIEC-6}

a)

C H DMOG DFO Tuni Thap AA DTT MG132

PORCN

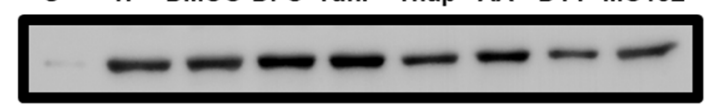

$\beta$-tubulin

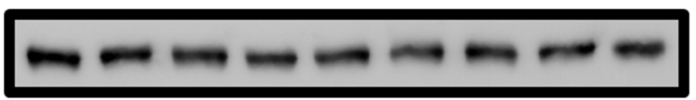

WLS

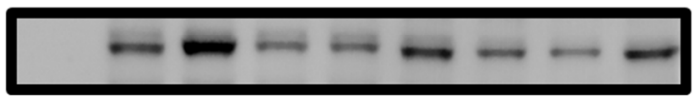

$\beta$-tubulin

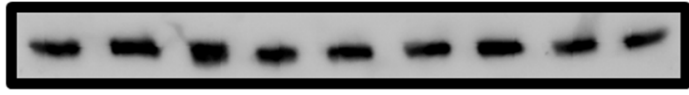

$\beta$-catenin

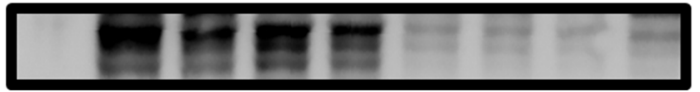

$\beta$-tubulin
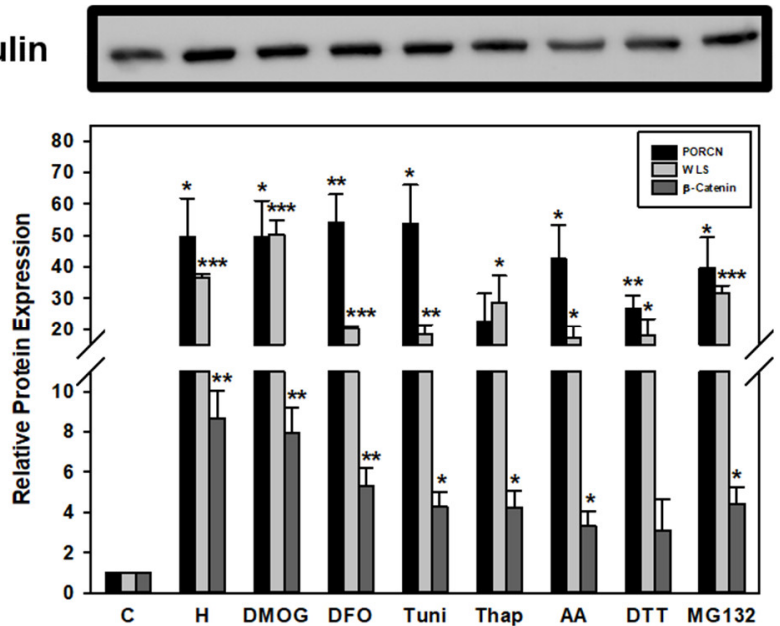

HCT116

b)
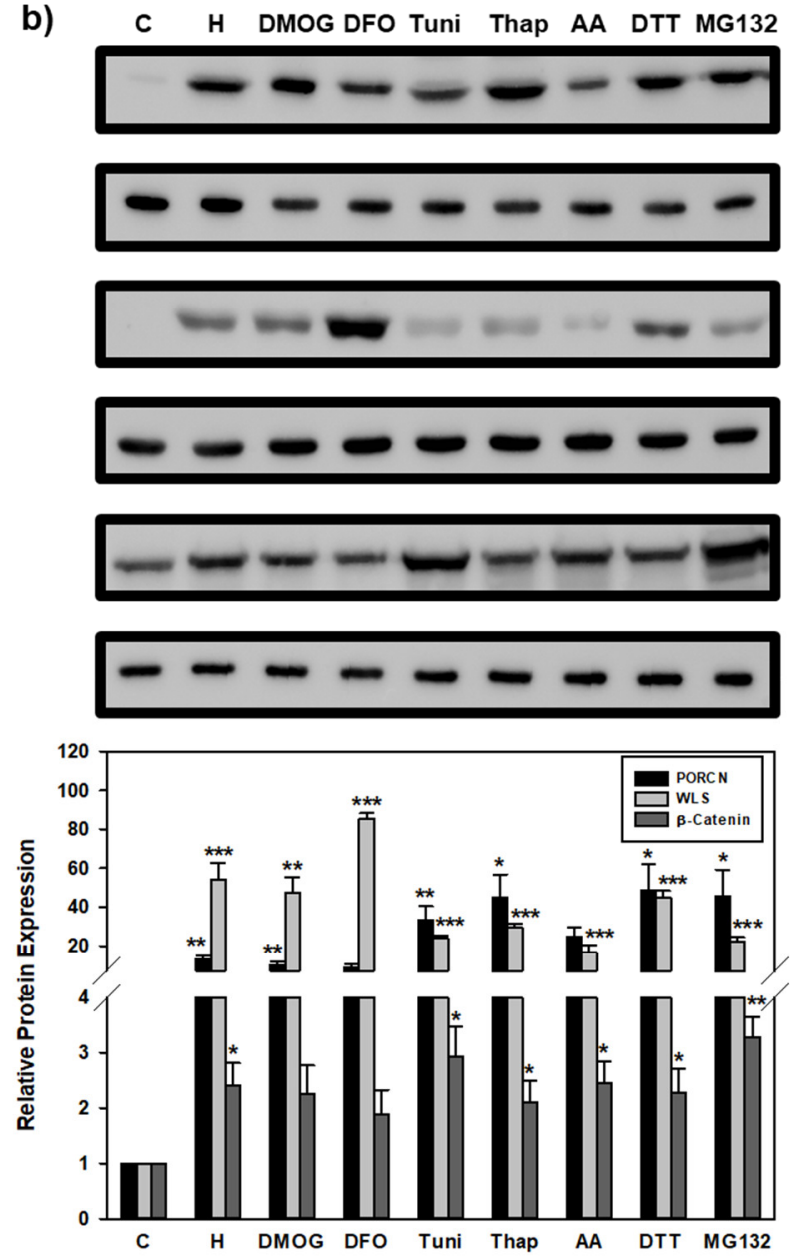

Figure 3.3: Western blot showing the effects of 24 hours of treatment on HIEC-6 and

HCT116 protein expression. Cultured HIEC-6 and HCT116 cells were treated with hypoxia ( $1 \% \mathrm{O}_{2}$ and $\left.5 \% \mathrm{CO}_{2}\right)$, hypoxic mimetics (100 $\mu \mathrm{M}$ desferrioxamine (DFO), 1 $\mathrm{mM}$ dimethyloxalylglycine (DMOG)), and ER stressors (5 mg/mL tunicamycin (Tuni), 3 $\mathrm{nM}$ thapisigargin (Thap), $90 \mathrm{mM}$ antimycin A (AA), $2 \mathrm{M}$ dithiothreitol (DTT)) for $24 \mathrm{hr}$ and $5 \mathrm{mg} / \mathrm{mL}$ MG132 for $3.5 \mathrm{hr}$. Cells without treatment were used as controls. Total membrane bound proteins were isolated using $8 \mathrm{M}$ urea. Samples were incubated with 
anti-WLS (65 kDa), anti-PORCN (55 kDa) or anti- $\beta$-catenin $(92 \mathrm{kDa})$ antibodies. The level of protein expression of each sample was normalized to $\beta$-tubulin. Data are the mean band densities from three independent experiment \pm S.E.M. Data analysis was done using one-way ANOVA followed by Dunnett's test; ${ }^{*} \mathrm{p}<0.05, * * \mathrm{p}<0.01, * * * \mathrm{p}<0.001$. 


\subsubsection{Electrophoretic mobility shift assay (EMSA) shows that PORCN and WLS}

genes under hypoxia, hypoxia mimetics, ER stressors and oxidative stressors induces the activation of HIF1 $\alpha$, NRF2 and NF- $\mathrm{KB}$ activation

Consensus DNA-binding sequences for HIF (HREs), NFE2L2 (AREs), and NF- $\kappa$ B (NF-kB-binding) sites were identified in the promoters of PORCN and WLS genomic sequences. A region approximately 10,000 b.p. from the start site of transcription (exon 1) was analyzed for putative response elements for both genes. The PORCN promoter contained one putative NF- $\mathrm{B}$ B-binding site $(-1652$ to -1662 b.p.), two putative AREs ( -1455 to -1465 b.p. and -533 to -543 b.p.) and one putative HRE ( -295 to -303 b.p.). Of these, the ARE at -1455 did not show binding in EMSAs and was abandoned (data not shown). The $W L S$ promoter contained two putative AREs ( -299 to -309 and -815 to -825 b.p.) and one putative HRE ( -648 to -656 b.p.). Of these, the ARE at -299 did not show binding in EMSAs and was abandoned (data not shown). EMSA was carried out on biotinlabelled binding sequences which corresponded to a 29-30 n.t. section of the promoter, flanking the binding site of the factor (HIF-1, NRF2, NF- $\kappa$ B). HEK293T whole cell extracts, taken from cells treated with hypoxia, hypoxic mimetics, ER stressors or oxidative stressors, were used in gel-shift assays to assess whether these transcription factors can bind to the promoter-specific sequence used in the binding assays. Treatments were assessed in HEK293T cells with and without overexpression of WLS (Fig. 3.4b). Without WLS overexpression, it was found that hypoxia, DMOG and DFO caused an increase in WLS protein expression (via Western blot) 5-fold, 4-fold and 3-fold, respectively.

In HIF-1 gel shift assays, two bands are often seen; the bottom band represents constitutive ("C") binding of HIF-1 to HREs and the top band represents induced ("I") 
binding of HIF-1 to HREs (Wang and Semenza, 1995). If both bands are seen to increase during treatment, this means the overall pool of HIF-1 protein has increased in the cell. To test the effects of enhanced levels of PORCN and WLS in cells on HIF-1, NRF2 and NF$\kappa \mathrm{B}$ binding to promoter elements, gel shift assays were undertaken using whole cell extracts from HEK293T, with or without overexpression of PORCN and WLS (treatments designated with a "(T)" are those that are overexpressing PORCN (a and d) or WLS (b and c) in Fig. 3.4). HREs were identified in both the PORCN and WLS promoters and the gel shifts in HEK293T cells for these promoter elements are shown in Figs. 3.4a and b, respectively. Increases in both induced and constitutive HIF-1 binding to HREs were seen with all treatments (hypoxia, DMOG and DFO), as well as transient transfection of cells with either PORCN (Fig. 3.4a, Lanes 1 and 2) or WLS (Fig. 3.4b, Lanes 1 and 2). However, only constitutive binding of HIF-1 to PORCN HRE promoter elements in hypoxia and DMOG treated cells overexpressing PORCN were significantly different from controls (Fig. 3.4a, Lanes 3 and 6). In HEK293T cells, induced binding of HIF-1 to WLS HRE promoter elements was significantly upregulated in hypoxia without overexpressing WLS (Fig. 3.4b, Lane 3). However, with overexpression of WLS, induced binding of HIF-1 was increased, but not significantly (Fig. 3.4, Lane 4). Induced and constitutive HIF-1 binding to WLS promoter HRE elements also occurred with both DMOG and DFO treatments (Fig. 3.4b, Lanes 5 and 6). In HEK293T cells overexpressing WLS, NRF2 binding to WLS ARE promoter elements significantly increased in the WLS promoter element in hypoxia (with and without overexpression of WLS), Tuni, Thap and AA by approximately 2.5 -fold for

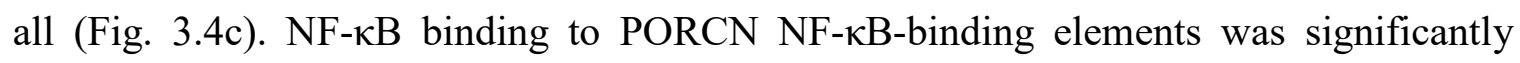


elevated in hypoxia (with and without PORCN overexpression), DMOG, DFO, Tuni AA, DTT and MG132 treatments by approximately 3-fold for all (Fig. 3.4d). 


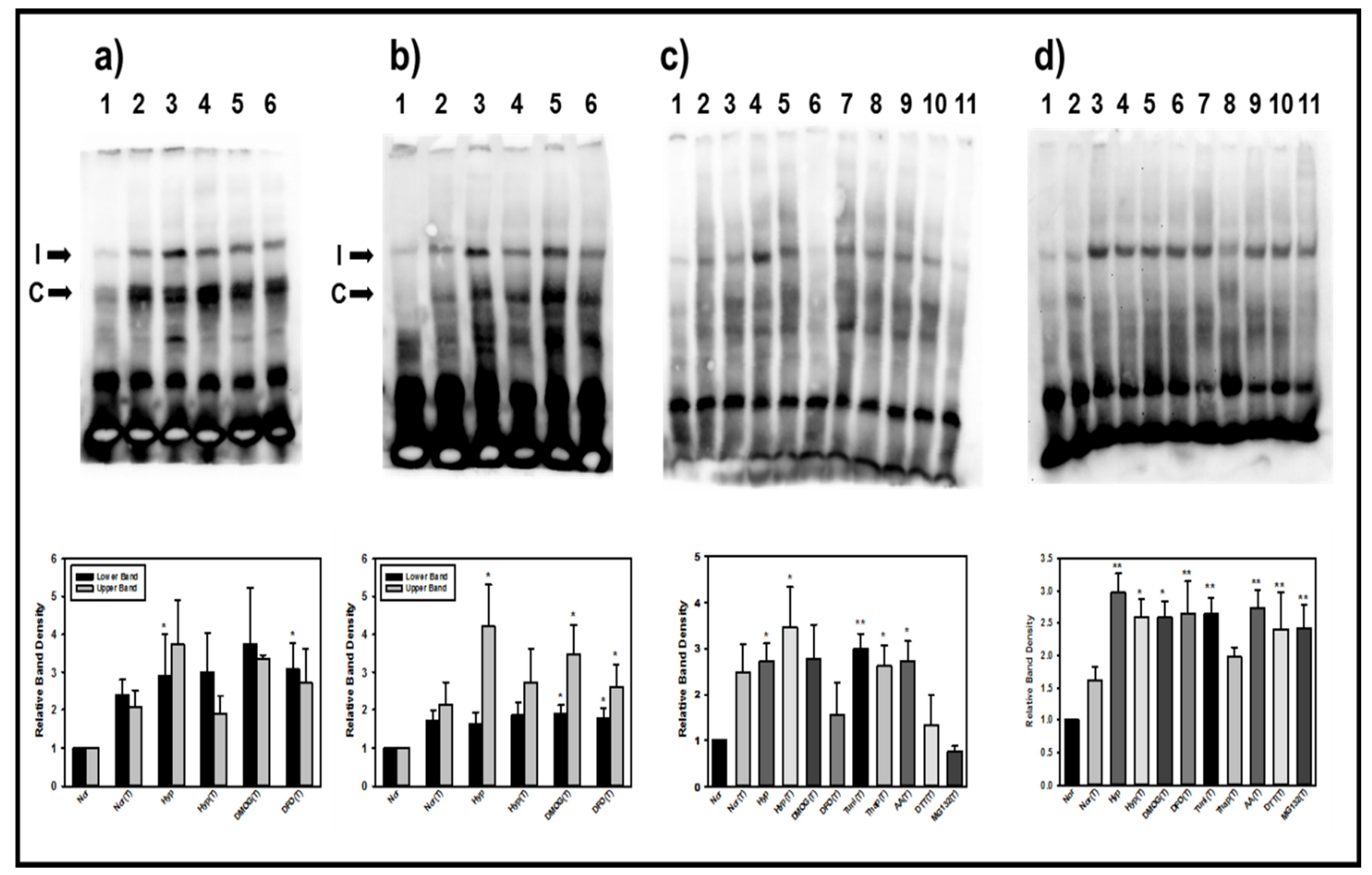


Figure 3.4: Effect of hypoxia, hypoxic mimetics (DMOG, DFO, MG132), ER stressors (Tuni, Thap, DTT, MG132) and oxidative stressors (AA) on HIF-1, Nrf2 and NF-אB DNA binding to PORCN and WLS promoter elements (a, b, c and d). Whole cell extracts were prepared and EMSA carried out of HIF-1 using HRE (consensus site) (a and b), Nrf2 using ARE (c) and NF-kB (d) labelled oligonucleotides corresponding to the consensus sites in PORCN and WLS promoter. Cells were overexpressed by PORC (a and d) and WLS (b and c) for 24 hrs. Overexpressed cells were treated with hypoxia $\left(1 \% \mathrm{O}_{2}\right.$ and 5\% $\left.\mathrm{CO}_{2}\right)$, hypoxic mimetics $(100 \mu \mathrm{M}$ desferrioxamine (DFO), $1 \mathrm{mM}$ dimethyloxalylglycine (DMOG)), and ER stressors (5 mg/mL tunicamycin (Tuni), $3 \mathrm{nM}$ thapisigargin (Thap), $90 \mathrm{mM}$ antimycin A (AA), $2 \mathrm{M}$ dithiothreitol (DTT)) for $24 \mathrm{hr}$ and $5 \mathrm{mg} / \mathrm{mL}$ MG132 for $3.5 \mathrm{hr}$. Cells without treatment were used as controls. The bottom band represents constitutive ("C") binding of HIF-1 to HREs and the top band represents induced ("I"). Data are the mean band densities from three independent experiment \pm SEM. Data analysis was done using one-way ANOVA followed by Dunnett's test; ${ }^{*} \mathrm{p}<0.05, * * \mathrm{p}<0.01, * * * \mathrm{p}<0.001$. 


\subsubsection{Knockdown of PORCN and $W L S$ using siRNA}

The PORCN (Fig. 3.5a) and WLS (Fig. 3.5b) genes were knocked down (KD) with si-RNAin HCT116 cells that were subsequently treated with hypoxia, hypoxia Knock down of both PORCN and $W L S$ was effective in reducing gene expression by $95-$ to $97 \%$. Under control conditions, hypoxia dramatically increased PORCN, WLS and WNT3A protein expression at detectable levels. With PORCN and $W L S$ down, PORCN, WLS and WNT3A was not expressed (Fig. 3.5), Overall, this suggests that both hypoxia WNT3A, WLS and PORCN, and that knock down of PORCN or $W L S$ does not restore levels of these proteins to non-knock down levels. 


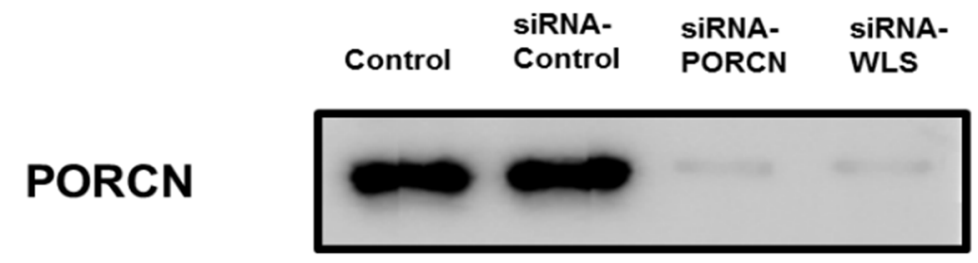

$\beta$-tubulin

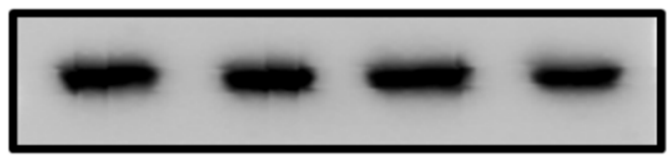

WLS

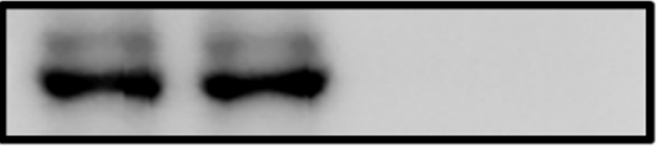

$\beta$-tubulin

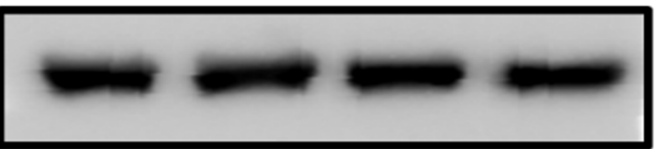

WNT3A

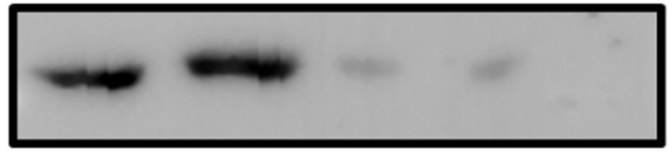

$\beta$-tubulin

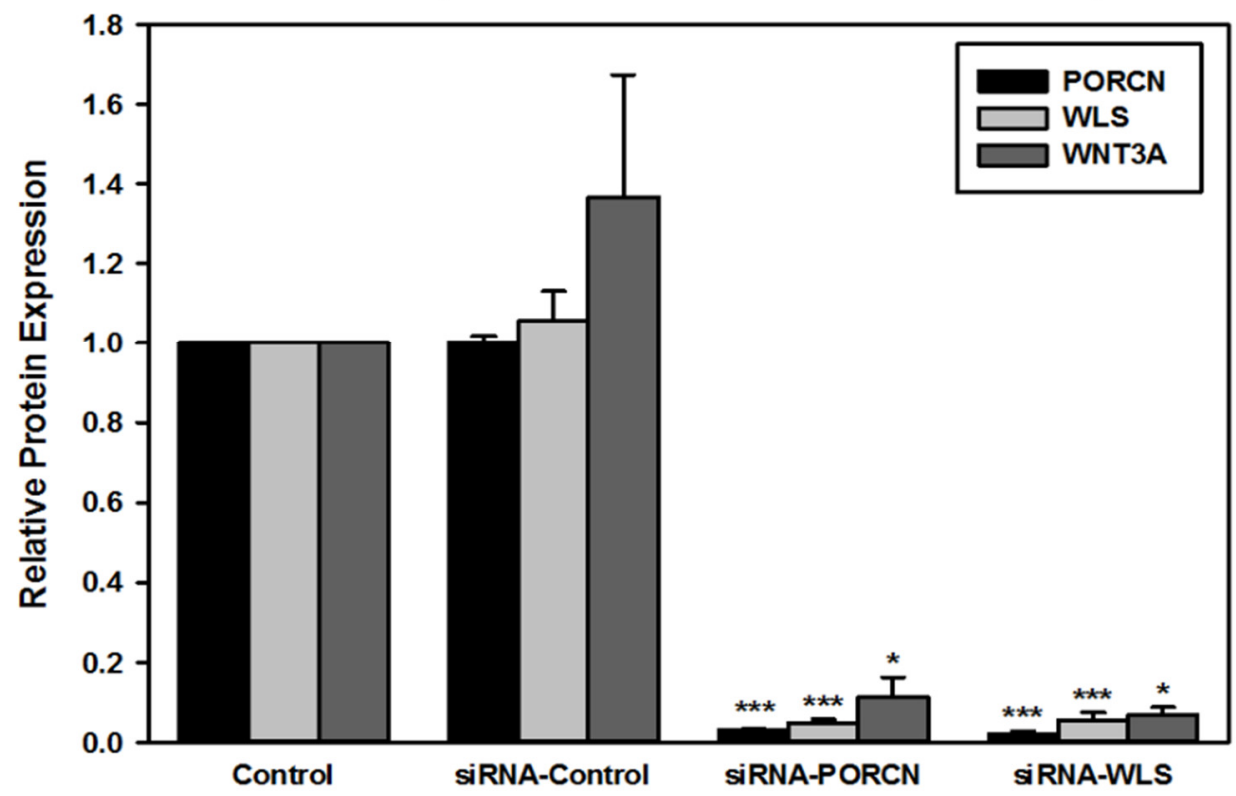


Figure 3.5: The siRNA knockdown of $P O R C N$ and $W L S$ genes were detected by Western blot analysis. HCT116 cells were transfected with PORCN and $W L S$ siRNA and Negative Control siRNA, and the levels of PORCN, WLS and WNT3A protein were detected by Western blot. The quantitative comparison of the difference of expression of PORCN, WLS, and WNT3A in each group. Total protein levels were normalized to levels of $\beta$ tubulin protein. The data are presented as the means \pm S.E.M. from at least 3 independent experiments $(* \mathrm{p}<0.05 ; * * \mathrm{p}<0.01 ; * * * \mathrm{p}<0.001$ by Dunnett's test). 


\subsubsection{Inhibition of palimatoylation using 2-bromohexadecanoic acid (2BHDA)}

We investigated palmitoyleation role in WNT secretory pathway by using an inhibitor of palmitoylation, 2-bromohexadecanoic acid (2BHDA) in HCT116 cells (Fig. 3.6). This experiment included the knock down of PORCN (PKD) and WLS (WKD) using dsRNAi. Panel a) shows that WLS knock down (WKD) and 2BHDA treatment inhibited WNT3A and WLS expression. WLS (WKD) showed no significant change in PORCN expression in both normoxic and hypoxic conditions. Panel b) shows that PORCN knock down (PKD) and 2BHDA treatment inhibited WNT3A, WLS and PORCN expression in both normoxic and hypoxic conditions. 


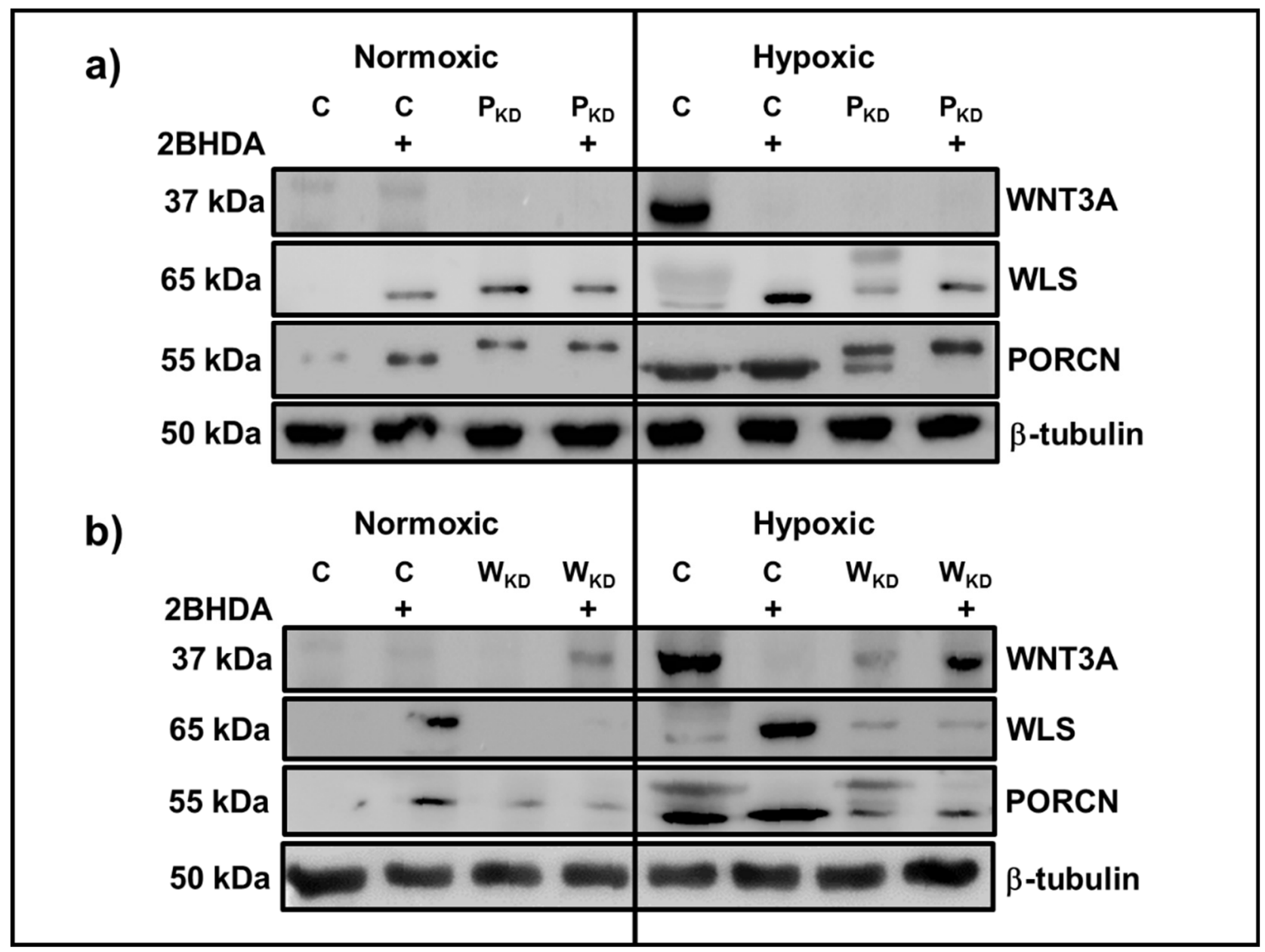

Figure 3.6: Effects of PORCN $\left(\mathrm{P}_{\mathrm{KD}}\right)$ and WLS ( $\left.\mathrm{W}_{\mathrm{KD}}\right)$ knock down and inhibition of palmitoyleation by 2-bromohexadecanoic acid (2BHDA) on the expression PORCN, WLS and WNT3A in HCT116 cells. Cells were transfected using siRNA targeting PORCN and WLS under normoxic and hypoxic (1\% oxygen) conditions. 


\subsubsection{PNGase F assay (deglycosylation of WNT proteins)}

We investigated the glycosylation process by completely deglycosylating PORCN, WLS and WNT3A with PNGase F, which cleaves off asparagine-linked glycans. This experiment included the knock down of PORCN (PKD) and WLS (WKD) using dsRNAi (Fig. 3.7). Panel a) shows that WLS knock down (WKD) and PGNase treatment inhibited WNT3A and WLS expression. WLS (WKD) showed a significant change in PORCN expression in both normoxic and hypoxic conditions. Panel b) shows that PORCN knock

down (PKD) and PGNase treatment inhibited WNT3A, WLS and PORCN expression in both normoxic and hypoxic conditions. 


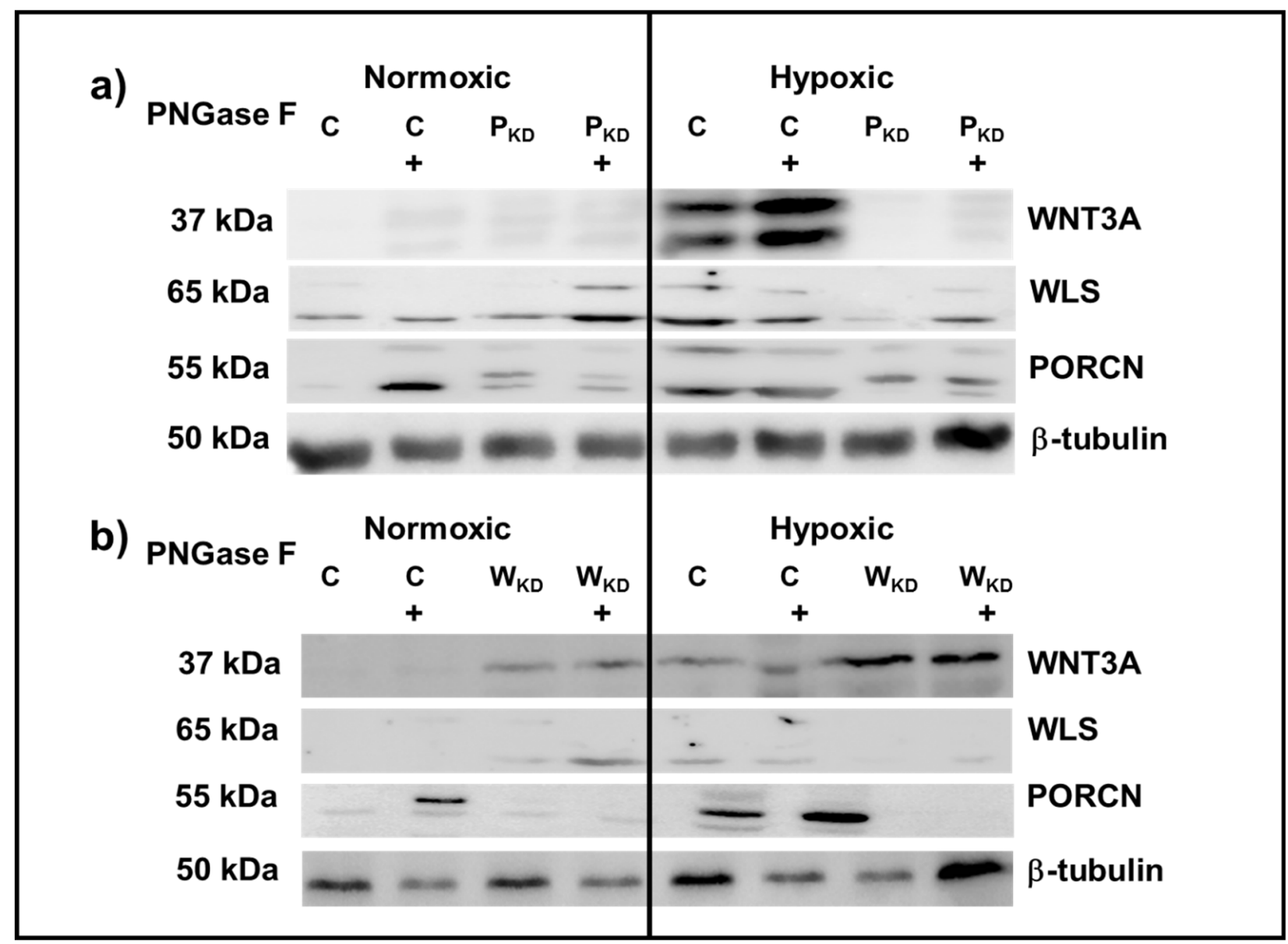

Figure 3.7: Effects of PORCN $\left(\mathrm{P}_{\mathrm{KD}}\right)$ and WLS $\left(\mathrm{W}_{\mathrm{KD}}\right)$ knock down and inhibition of $\mathrm{N}$ glycosylation by F-PGNase on the expression PORCN, WLS and WNT3A in HCT116 cells. Cells were transfected using siRNA targeting PORCN and WLS under normoxic and hypoxic ( $1 \%$ oxygen) conditions. 


\subsubsection{WNT3A response are activated upon exposure to hypoxia and hypoxia mimics, oxidative and ER stressors}

To investigate the role of low oxygen on WNT3A expressions, HCT116 and HIEC6 cells were treated with hypoxia $\left(1 \% \mathrm{O}_{2}\right)$, or the hypoxic mimetics DMOG or DFO, for 24 hrs. Hypoxia, DMOG and DFO strongly induced WNT3A protein in both cell lines (Fig. 3.8). However, the $W N T 3 A$ gene expression was significantly elevated when treated with hypoxia and DFO (Fig 3.9). Western blot revealed the presence of WNT3A (45 kDa), in HIEC-6 and HCT116 cells, but primarily when induced by treatment. Expression of WNT3A protein was low in non-treated cells. Treatments with Thap, AA, DTT and MG132 resulted in a higher protein expression in HCT116 and HIEC-6 (Fig. 3.8).

Induction of $W N T 3 A$ gene expression was also seen higher with Thap, AA, DTT and MG132 (Fig. 3.9). However, treatment with Tuni showed much lesser effect (Fig. 3.9). 


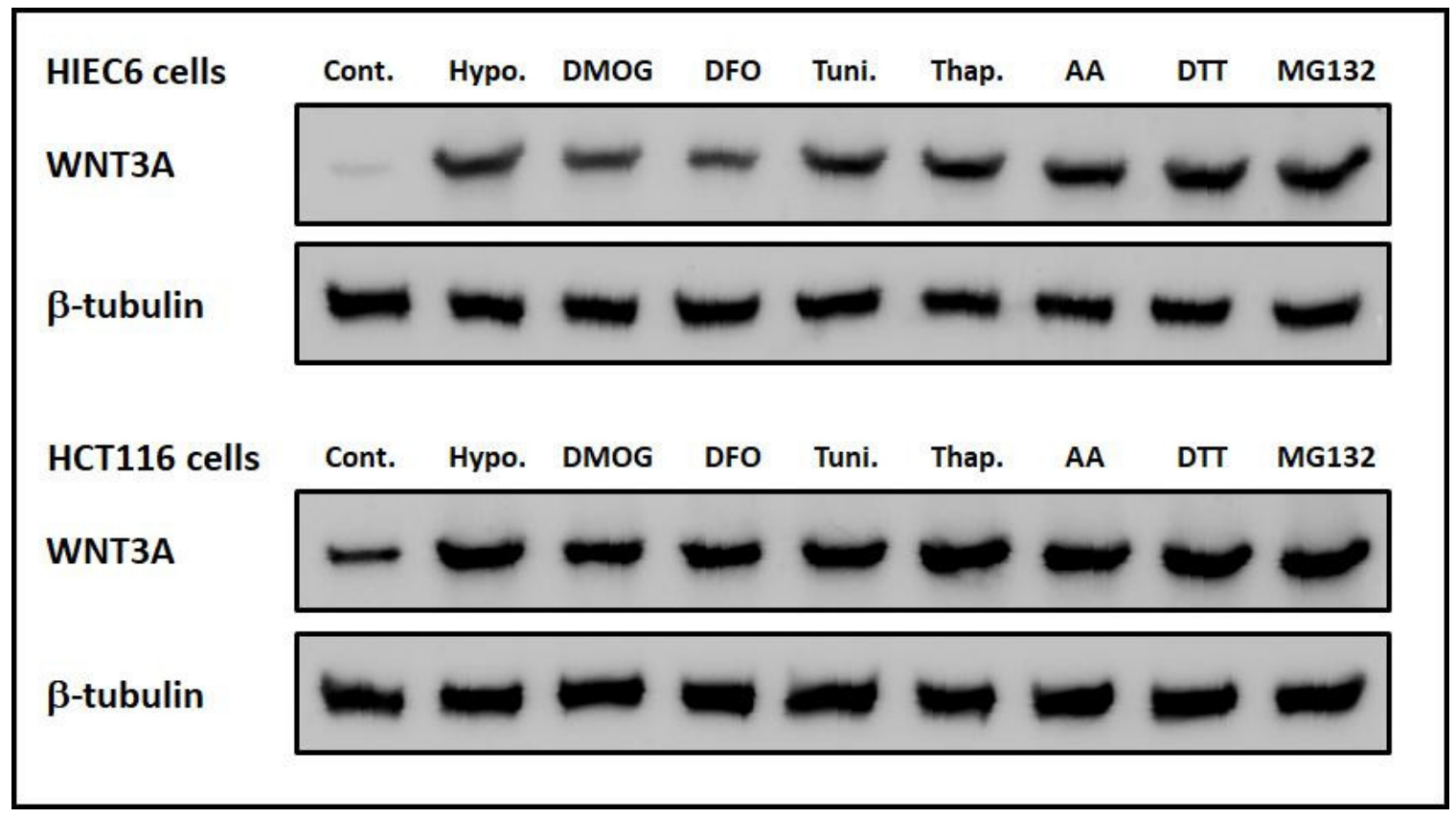

Figure 3.8: Western blot showing the effects of 24 hours of treatment on HIEC-6 and HCT116 protein expression. Cultured HIEC-6 and HCT116 cells were treated with hypoxia ( $1 \% \mathrm{O}_{2}$ and $5 \% \mathrm{CO}_{2}$ ), hypoxic mimetics (100 $\mu \mathrm{M}$ desferrioxamine (DFO), $1 \mathrm{mM}$ dimethyloxalylglycine (DMOG)), and ER stressors (5 mg/mL tunicamycin (Tuni), $3 \mathrm{nM}$ thapisigargin (Thap), $90 \mathrm{mM}$ antimycin A (AA), $2 \mathrm{M}$ dithiothreitol (DTT)) for $24 \mathrm{hrs}$ and $5 \mathrm{mg} / \mathrm{mL}$ MG132 for $3.5 \mathrm{hrs}$. Cells without treatment were used as controls. Total membrane bound proteins were isolated using $8 \mathrm{M}$ urea. Samples were incubated with antiWNT3A (45 kDa) antibodies. The level of protein expression of each sample was normalized to $\beta$-tubulin. Data are the mean band densities from three independent experiment \pm S.E.M. Data analysis was done using one-way ANOVA followed by Dunnett's test; $* \mathrm{p}<0.05, * * \mathrm{p}<0.01, * * * \mathrm{p}<0.001$. 


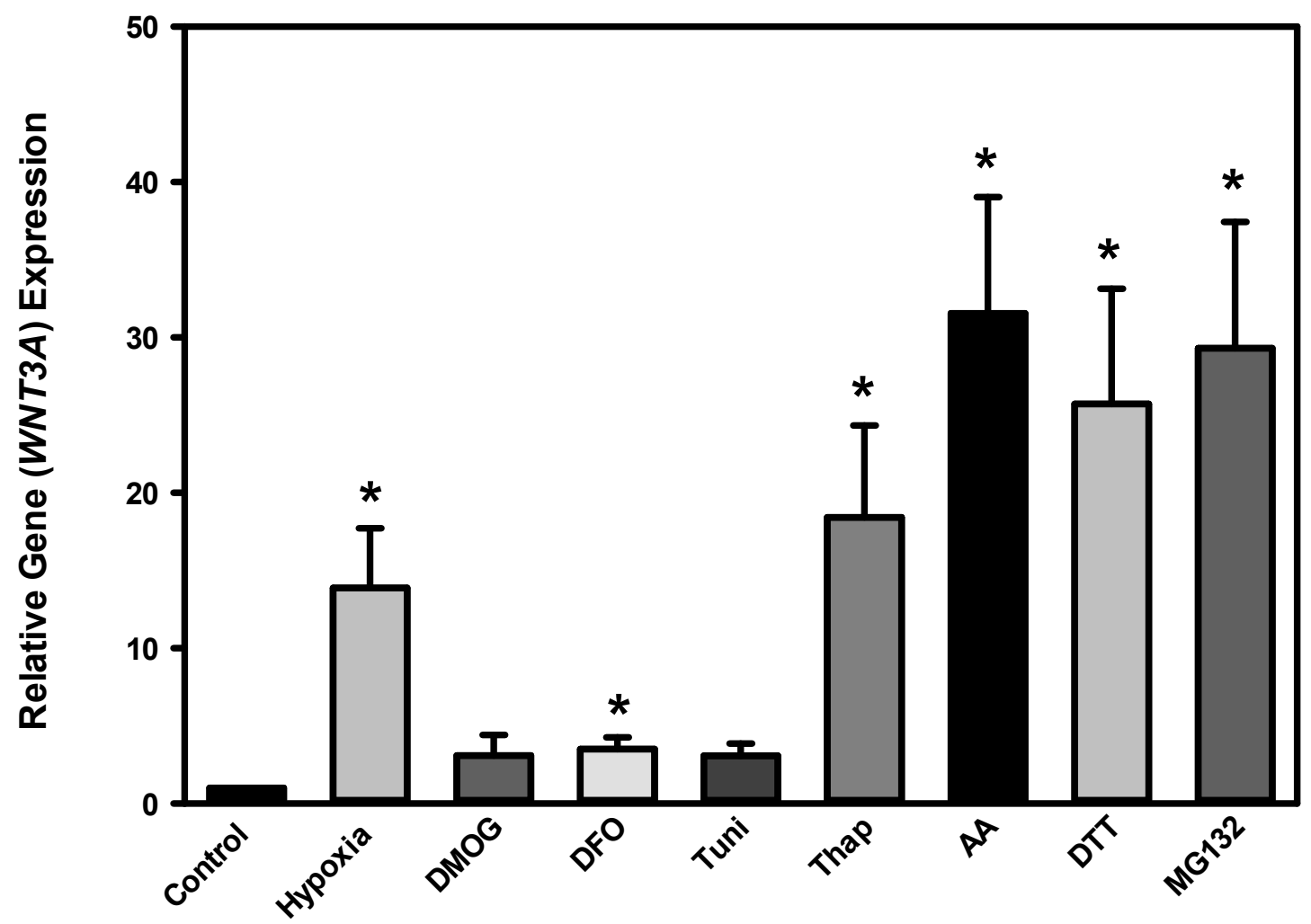

Figure 3.9 The effect of hypoxia and hypoxic mimetics, ER stressors and oxidative stressors on WNT3A gene expression in human colorectal carcinoma (HCT116) cells. HCT116 cells were treated with hypoxia $\left(1 \% \mathrm{O}_{2}\right.$ and $\left.5 \% \mathrm{CO}_{2}\right)$ and hypoxic mimetics $(100$ $\mu \mathrm{M}$ desferrioxamine (DFO) and $1 \mathrm{mM}$ dimethyloxalylglycine (DMOG), $(5 \mathrm{mg} / \mathrm{mL}$ tunicamycin (Tuni), $3 \mathrm{nM}$ thapsigargin (Thap), $90 \mathrm{mM}$ antimycin $\mathrm{A}$ (AA), $2 \mathrm{M}$ dithiothreitol (DTT) for $24 \mathrm{hrs}$ and $5 \mathrm{mg} / \mathrm{mL}$ MG132 for $3.5 \mathrm{hrs}$. Cells without treatment were used as controls. Total RNA was isolated from HCT116 cells, converted to cDNA and analyzed by qPCR using gene-specific primers for WNT3A. The level of target genes of each mRNA was normalized to the GAPDH mRNA. Primers used for WNT3A gene expression were 5'-GACTTCCTCAAGGACAAGTACG-3' (forward) and 5'- 
GGAACCTTGAAGTAGGTGTAG-3' (reverse). Data are the average from three independent experiments \pm S.E.M. Data analysis was done using one-way ANOVA followed by Dunnett's test; *p $<0.05$. 


\subsection{Discussion}

The secretion of WNT, in response to stress, plays important roles in biological and pathophysiological processes. Many studies on intestinal tumorgenesis focus on the downstream events of WNT proteins binding to the Frizzled-LRP5/6 receptors and the canonical pathway of signal transduction activated by WNTs in the recipient cell. They mainly focus on the APC protein (which associates with axin and the kinases GSK3 and CK1 to target $\beta$-catenin for destruction) and $\beta$-catenin itself. Few studies examine the upstream pathway of WNT modification, which are essential for the protein's binding to the Frizzled-LPR5/6 receptors. Ours is the first study to examine how conditions in the tumour microenvironment (hypoxia, oxidative stress, ER stress) affect the expression of these proteins, as well as on the model WNT protein, WNT3A. In this study, we investigated the role of oxidative stress, ER stressors, hypoxia, and hypoxic mimetics in altering gene and protein expression in WNT secretory pathway. The links between stress response and WNT secretion remain poorly understood. Hypoxic, oxidative and ER stresses are known to activate different signaling cascades, but their effects on the WNT secretion pathway have not been studied.

\subsubsection{The effect of hypoxic conditions on gene and protein expression of PORCN, WLS and $\beta$-catenin in vitro}

Many hypoxic responses are activated by Hypoxia-Inducible Factors (HIFs) (Muz et al., 2015; Wang et al., 2015) and we investigated whether hypoxia activates genes in the WNT production pathway, as well as the possibility that this hypoxic activation could be regulated by HIFs. Putative Hypoxia Response Elements (HREs) were identified in the 
promoters of the PORCN and WLS genes (Fig.2.1). Both hypoxia and hypoxic mimetics induced PORCN (hypoxia, DMOG and DFO), WLS (hypoxia and DMOG) and WNT3A (hypoxia and DFO) gene expression (Fig. 3.2a and b respectively and 3.9), compared to normoxic controls in HCT116 cells. Hypoxia and hypoxic mimetic treatments also resulted in large inductions of PORCN, WLS and WNT3A protein expression (Fig. 3.3, right panels and 3.8) in both HIEC-6 and HCT116 cells; however, only protein changes in WLS were significant in HEIC-6 cells. DFO had a stronger induction of WLS protein than hypoxia or DMOG, in both cell lines, which suggests that iron chelation may have secondary effects, outside of hypoxia and HIF regulation that controls WLS expression. DFO did not have an effect on PORCN protein expression in HCT116 cells. DFO is an iron chelating agent which acts as a hypoxic mimetic by inhibiting iron-dependent prolyl hydroxylases that hydroxylate Hypoxia-Inducible Factors (HIFs) in an oxygen-dependent manner. Iron chelation may have a stabilizing effect on the WLS protein (translational or posttranslational control) and may not be the result of increases in WLS gene expression (transcriptional control). This would suggest that WLS gene expression is not under the control of HIF proteins (which are regulated in an oxygen- and iron-dependent manner) and that the effects of DFO are either directly upon WNT proteins, or through an interacting protein with the WNTs. In the absence of all stressors utilized in this study, the expression of PORCN, WLS, WNT3A, and $\beta$-catenin proteins was low.

$\beta$-catenin protein also showed higher expression with hypoxia, but not DMOG or DFO, in HCT116 cells. $\beta$-catenin accumulates when the WNT proteins are activated and bind to FZD (Katoh, 2015; Kim et al., 2012). In this study, induction of $\beta$-catenin by hypoxia may be a direct result of the hypoxic induction of WNTs (Fig. 3.4) which, in turn, 
is induced by upregulated PORCN and WLS (Fig. 3.3). The induction of WNT processing by hypoxia causes a general upregulation of $\beta$-catenin signaling. In canonical WNT signaling, progressive accumulation of unphosphorylated $\beta$-catenin in the cytoplasm is translocated into the nucleus and acts as a transcriptional co-activator of transcription factors, including the $\mathrm{T}$ cell factor-lymphoid enhancer factor (TCF-LEF) family of proteins (MacDonald et al., 2009). Our results showed that HCT116 cells, when treated with hypoxia, exhibited higher levels of WNT/ $\beta$-catenin protein expression. Previous work indicated that hypoxia destabilizes $\beta$-catenin through endogenous degradation machinery (Verras, et al., 2008). These results show that hypoxia may interfere with the machinery responsible for the posttranslational modification of WNT proteins, causing the UPR and altering the efficiency of WNT secretion.

WNT3A gene expression were not altered by DFO and DMOG compared to hypoxia in (Fig. 3.2). Low oxygen stimulates airway branching and involves hypoxia-inducible factor (HIF). HIF is stable and initiates expression of angiogenic factors under hypoxia. This can trigger a much greater induction throughout the cell. Whereas normoxia triggers hydroxylation of the HIF-1alpha subunit by prolyl hydroxylases (PHDs) and subsequent degradation usually done by a hypoxia mimetic treatment such as DMOG and DFO (Groemann et al., 2007). DFO creates hypoxia by chelating irons and altering the iron status of iron- and $\mathrm{O}_{2}$-dependent hydroxylases, from which HIF receives the cellular $\mathrm{O}_{2}$ level information. DFO has also been shown to increase HIF-1 gene expression in cells. Our results showed that treatment with DFO-induced hypoxia specifically affects protein expression at post-transcriptional level no at the transcriptional level (Wang and Semenza, 1995). DMOG treatment decreases NADH levels and inhibits respiration in cancer cells, 
decrease in respiration precedes and does not depend on activation of HIF signalling (Zhdanov et al., 2015).

In gel-shift assays, there was both increased induced (I) binding and constitutive (C) binding to HREs in both the PORCN and the WLS promoter regions (Fig. 3.4a and b respectively). Increased I and C HRE binding was also seen with treatments with hypoxic mimetics, including DFO for PORCN and DMOG and DFO for the HRE found in the WLS promoter element. Overall, this suggests that HIF plays an integral role in the hypoxic expression of both PORCN and WLS proteins. HIF activity is regulated by epigenetic alterations and mutations, causing a loss of tumour-suppressor function and a gain-ofoncogenic function (Muz et al., 2015). It may also be involved in regulating ER stress responsive genes (Koumenis C, Wouters, 2006). Previous studies have shown that DMOG is a competitive inhibitor of prolyl hydroxylase domain (PHD)-containing proteins, as well as an activator and stabilizer of HIFs that not only regulate hypoxic gene expression but also regulate calcium and sodium homeostasis, as well as pro-inflammatory responses (Iommarini, et al., 2017). DFO functions by depleting iron and inactivating prolyl hydroxylases, which mimics the hypoxic condition (Chaston et al., 2003). Interestingly, increased binding to an ARE in the $W L S$ promoter, as well as a NF- $\mathrm{KB}$ response element in the PORCN promoter, also increased with hypoxia (Fig. 3.4c and d). Increased binding to the NF- $\mathrm{kB}$ response element of PORCN was also seen with DMOG and DFO treatments (Fig. 3.4d). This result suggests that hypoxia induction of the NFE2L and NF- $\mathrm{BB}$ proteins may, in part, have a role in the hypoxic expression of the PORCN and $W L S$ genes and proteins seen in this study. Our results support that the gene and protein expression of WLS 
and PORCN are altered by hypoxia and hypoxic mimetics. The expression of $W L S$ and PORCN genes may be transcriptionally regulated by oxygen through the binding of HIFs to HREs, as well as NFE2L proteins to AREs and NF- $\mathrm{KB}$ to its response element.

\subsubsection{The effect of ER and oxidative stressors on gene and protein expression of PORCN, WLS, WNT3A and $\beta$-catenin in vitro}

Many antioxidant responses and ER stress responses are activated by the nuclear factor erythroid 2-related factors (NFE2L1, NFE2L2 and NFE2L3) which heterodimerize with the small MAF proteins (MAFF, MAFG, MAFK) and bind to the Antioxidant Response Element (AREs). An analysis of the PORCN and $W L S$ promoter regions (Fig. 1a and $\mathrm{b}$, respectively) revealed two putative AREs for the PORCN gene (-533 to -543 and -1455 to -1465 from the start site of transcription) and two putative AREs for the WLS gene ( -299 to -309 and -815 to -825 b.p. from the start site of transcription). Of these, one ARE for PORCN (-1455 b.p.) and one ARE for WLS ( -299 b.p.) showed no binding in EMSAs and were not tested further.

Gene expression of PORCN, WLS and WNT3A in HCT116 cells was assessed by qPCR using a comprehensive panel of ER stressors (tunicamycin (Tuni), thapsigargin (Thap), dithiolthreitol (DTT), proteasome inhibition (MG132)) and oxidative stressors (antimycin A (AA)). Notably, mRNA expression was significantly elevated in HCT116 cells after treatment with Thap, AA and MG132 for PORCN and WNT3A and after treatment with Tuni, Thap, AA and MG132 for $W L S$ (Fig. 3.2c and d respectively, 3.9). Tuni blocks the formation of N-glycosylation in proteins, resulting in ER stress (Shen et al., 2015). Deglycoylation was performed using F-PGNase inhibitors (Fig. 3.7). The data 
indicated that glycosylation is very essentional for PORCN, WLS and WNT3A expression (Fig 3.7). Thap is non-competitive inhibitor of the sarco/endoplasmic reticulum calcium ATPase, the treatment of which causes the depletion of ER calcium stores, leading to ER stress (Oslowski and Urano,, 2011). The mechanisms by which Tuni (WLS) and Thap (PORCN and WLS) induce the expression of the WNT modifying enzymes remain to be determined. The transcription factor NFE2L1 has previously been shown to respond to both oxidative stress and ER stressors (Zhu et al., 209) and, given that binding to AREs was found in this study, it is possible that this factor controls the expression of both PORCN and $W L S$.

This study is the first to show that proteasome inhibition with MG132 results in the highest PORCN, WLS and WNT3A gene expression, compared to the other treatments (Fig. 3.2c and d respectively and 3.9). The mechanism by which MG132 increases $W L S$ and PORCN gene expression, however, is not known. MG132 has been previously shown to upregulate the stress-inducible factor ATF3 (Zimmermann et al., 2000). MG132 also prevents the proteolytic degradation of the alpha subunits of hypoxia-inducible factors (HIFs) (Shin et al., 2007). The NFE2L transcription factors (NFE2L1, NFE2L2 and NFE2L3) are also known to be ubiquitinated and degraded by the proteasome (Chepelev, et al., 2011; Stewart et al., 2003; Nouhi et al., 2007). The ubiquitination of mitochondrial proteins with proteasome inhibition, and the subsequent induction of ER stress (Mårtensson, et al., 2019), is another possible mechanism by which MG132 stabilizes WLS and PORCN. Further studies need to explore the effect of ER stressors and mitochondrial function on the WNT pathway. 
The effects of the ER stressors (Tuni, Thap, DTT, and MG132) and inducers of oxidative stress (AA), on PORCN, WLS and $\beta$-catenin protein expression were tested in HIEC-6 and HCT116 cells (Fig. 3.3, left side panels). Previous studies indicate that treatment with DTT results in increased gene expression of ER stress-related transcription factors, such as stress-Activated Transcription Factor 6 (ATF6). AA induces the production of free radicals from mitochondrial electron transport chain (ETC) complex III, causing oxidative stress (Nadanaka, et al., 2004). The WNT8A and WNT1A genes are known to be induced with AA treatment (Mihara et al., 2016). With the exception of AA treatment in HCT116 cells, PORCN protein expression was significantly higher than controls for all treatments, in both cell lines. Likewise, with the exception of AA and DTT treatments in HIEC-6, WLS protein expression was significantly higher than controls for all treatments, in both cell lines. As well, all treatments significantly increased $\beta$-catenin expression in HCT116 cells. In the absence of WNT stimulation, $\beta$-catenin is phosphorylated within the destruction complex by the serine/threonine kinases CK1 and GSK3 (Logan and Nusse, 2004; Coleman and Haller, 2019). Phosphorylated $\beta$-catenin is then recognized by $\beta$-TrCP, a component of an E3 ubiquitin ligase complex, and ubiquitinated (Ub) within the destruction complex. Subsequently, ubiquitinated $\beta$-catenin is rapidly degraded by the proteasome. The removal of $\beta$-catenin from the destruction complex is accomplished simply by direct degradation by the proteasome. Thus, both ER stress and oxidative stress upregulated all proteins within the pathway and this response appeared to be conserved between non-cancer and cancer cell lines. This may have been, in part, due to the increased gene expression of PORCN, WLS and WNT3A (Fig. 3.2c and d respectively) which are regulated by the factors examined in this study. 
Gel-shift assays showed an increased binding to an ARE found in the $W L S$ promoter in response to Tuni, Thap and AA treatments (Fig. 3.4c). They also showed an increased binding to a NF- $\mathrm{kB}$ element found in the PORCN promoter in response to Tuni, AA, DTT, and MG132 treatments (Fig. 3.4d). These results suggest that the NFE2L proteins play a role in $W L S$ gene expression in response to ER stress and oxidative stress. They also suggest that NF- $\kappa \mathrm{B}$ has a function in $P O R C N$ gene expression in response to the same stresses.

\subsubsection{The effect of PORCN and WLS knock down on PORCN, WLS and WNT3A protein expression in vitro}

We tested the effects of PORCN and WLS knock down on the expression PORCN, WLS and WNT3A proteins in HCT116 cells. WNT3A, WLS, and PORCN all showed a strong induction with hypoxia that was knocked down with PORCN and WLS RNAi in both cell lines utilized (Fig. 3.5).

Hypoxia and oxidative stress lead to ER stress, which plays an essential role in the control of homeostasis of the intestinal epithelium thus triggering an inflammatory signal that initiate proliferation and innate immune responses which have implications in gastrointestinal diseases (Coleman and Haller, 2019; Wang et al., 2014). The possibility exists that hypoxia-, oxidative stress- and ER stress-inducible factors control the expression of the proteins responsible for modifying the WNT proteins prior to their secretion from the cell. Altered WNT modification has been shown to affect proper WNT folding, secretion and binding to FZD (Ho and Keller,2015; Boone et al; 2014). Our results show changes in both the expression and modification of the PORCN and WLS proteins occurs 
under different stress conditions in two different cell lines. Induction by hypoxia, oxidative stress, and ER stress was seen in this study, at both the gene and protein levels, for both PORCN and WLS. Here we show that this may be through stress-induced transcription factors, such as HIF, NFE2L, and NF-kB. Hypoxia, oxidative stress, and ER stress also showed a strong induction of $\beta$-catenin (Fig. $4 \mathrm{~b}$ and d). The hypoxic induction of $\beta$-catenin correlated with a strong induction of WNT3A with hypoxia (Fig. 3.5a and b).

AA treatment showed significant results for both WLS and PORCN in both cell lines (Fig. 3.3). To the best of our knowledge, this finding was the first to demonstrate how endogenously generated oxidative stress can elevate the expression of the PORCN and WLS protein expression. Little is known about mitochondrial-generated ROS and the expression of PORCN and WLS. Interestingly, overexpression of WLS and PORCN, in conjunction with AA treatment, showed higher ARE and NF-kB response element binding than controls (Fig. 4). AA generated ROS in mitochondria will result in the UPR being generated in the ER.

\subsubsection{The effects of PORCN and WLS knock down on WNT3A expression}

PORCN knock down abrogated WNT3A expression, especially under hypoxic conditions, under which WNT3A is highly induced (Fig. 3.5a). The same was seen with WLS knock down (Fig. 3.5b). Our results show that knock down of PORCN and WLS affect WNT3A secretion.

In previous studies, hypoxia has been found to induce both oxidative stress and ER stress (Chipurupalli et al., 2019). Protein folding is dependent upon environmental concentrations of oxygen, the lack of which leads to the accumulation of 
misfolded/unfolded proteins (Koritzinsky, et al., 2013). Disulfide bonds introduced rapidly during protein synthesis can occur without oxygen, whereas those introduced during posttranslational folding or isomerization are oxygen dependent.

The WNT proteins have a large abundance of cysteines (6-7\% compared to 3.3\% for the average vertebrate protein), with a high abundance of cysteines towards the $\mathrm{C}$-terminus of the proteins. Redox regulation of the WNT proteins remains a possibility during these stresses.

\subsubsection{Palmitoyleation is necessarily for WNT secrection}

Palmitoylated WNT proteins were found throughout the cytosol and at the plasma membrane. PORCN itself and as PORCN is palmitoylated itself and this may be affected by these stresses such as hypoxia (Fig 3.6). Alterations in the expression of PORCN (either protein or gene) will potentially cause change in palmitoyleation of the WNT proteins. Our results indicated that palmitoyleation is necessary for WNT3A secretion (Fig. 3.6). PORCN can transfer monounsaturated and the main factor is the presence of stearoyl desaturase ( $\mathrm{SCD}$ ) as the enzyme responsible for generating the monounsaturated fatty acid substrate for PORCN. Our hypothesis that by knocking down PORCN or inhibiting the palmitoyleation step it can ultimately inhibit the SCD as an alternative mechanism for blocking WNT pathway activation (Fig. 3.6).

\subsection{Conclusions}

In summary, the effects of stress on WNT signaling pathways may be through key transcription factors known to be induced by stress (HIF through hypoxia; NFE2L1 
through oxidative and ER stresses and NF- $\mathrm{kB}$ in inflammatory stresses). Other coactivators and transcription factors involved in ER stress, such as ATF6, GRP78/BiP, XBP1 and ER stress-associated pro-apoptotic factors, may also influence WLS and PORCN expression. These ER stress-inducible factors are known to play a key role in the development of colon cancer. Understanding how WLS and PORCN gene and protein expression are altered under these stress conditions will provide insight into how their functions are altered in the tumour microenvironment and how this, in turn, affects the secretion of the WNT proteins. The possibility exists that PORCN specifically may be involved in a novel signaling pathway, independent of its palmitoleation of the WNT proteins and its role in their secretion, that is rate-limiting for cancer cell growth and tumorigenesis within the tumour microenvironment, as was suggested previously (Covey et al., 2012). Future studies will focus on the role of stress-inducible factors in WNT signaling and its involvement in cell proliferation and differentiation. 


\section{Chapter 4: Transcription factor control of WNT signalling pathway components}

\subsection{Introduction}

The WNT/ $\beta$-catenin pathway is conserved throughout the animal kingdom and is essential for embryonic patterning, cell fate determination, proliferation, and tissue homeostasis (Polaskis, 2012). Aberrant activation of WNT $\beta$-catenin signaling is linked to several diseases, eg. many cancers (Holland et al., 2013; Polaskis, 2012; Komiya and Habas, 2008). When secreted WNT factors bind to their receptors, a set of biochemical changes is set in motion, ultimately resulting in the accumulation of $\beta$-catenin and its translocation to the nucleus. In the nucleus, $\beta$-catenin binds to members of the TCF/LEF family of transcription factors, the most downstream components of the WNT-signaling pathway (Clevers, 2006).

In WNT-producing cells, the WNT protein becomes palmitoylated in the ER by the PORCN acyltransferase. Furthermore, transport and secretion of the WNT protein in secretory vesicles is controlled by the multi-pass transmembrane protein WNTLess/Evi, which is present in the Golgi and/or on the plasma membrane. The retromer complex, including VPS35, may act within WNT-producing cells to generate WNT forms that can be transported outside cells, possibly in the form of a lipoprotein particle (Takada et al., 2006).

A role for PORCN, as a WNT acyltransferase, is to recognize the protein substrate to which the fatty acid is attached. All studies of WNT acylation to date have relied on labeling cells with the saturated fatty acid palmitate (Chen et al., 2009; Doubravska et al., 
2011; Komekado et al., 2007; Takada et al., 2006; Willert et al., 2003), palmitoleate (palmitoleic acid; an 18-carbon chain, single double bond containing fatty acid) is the major fatty acid attached to the WNT proteins (Takada et al., 2006). Studies showed that stearoyl-CoA desaturase 1 (SCD1) is responsible for generating the monounsaturated fatty acid (MUFA) substrate for PORCN. It's known that SCD1 is an ER-resident protein and that it is the rate-limiting enzyme in the biosynthesis of MUFAs from saturated fatty acid precursors. It introduces a cis-double bond at position 9 of palmitoyl-CoA (16:0) and stearoyl-CoA (18:0) to generate palmitoleoyl-CoA (16:1) and oleoyl-CoA (18:1), respectively (Miyazaki et al., 2006; Ntambi and Miyazaki, 2004). In the absence of SCD1 activity, endogenous MUFA production is decreased, resulting in reduced lipid accumulation due to decreased fatty acid synthesis and increased fatty acid oxidation resulting in a series of stresses (Ntambi and Miyazaki, 2003).

Cells have evolved a series of integral systems to protect against various stresses. As such, living organisms adapt to the changing environments during physiological and pathological stresses (Sporon et al., 2012). Hence, it is plausible that there exists at least a set of versatile defense mechanisms (e.g., redox signaling to antioxidant gene regulatory networks against oxidative stress ( $\mathrm{Li}$ et al., 2019). Cancer cells attack normal cell machinery by activating growth factor pathways to sustain cellular growth and proliferation (Hannah and Weinbergs, 2011). This permits cancer cells to uptake nutrient and survive stress conditions. As a result, the hyper-metabolic state of cancer cells causes the generation of stress from the mitochondria and endoplasmic reticulum (Carins et al., 2011). We chose a set of a transcription factors that are activated by common stresses found within the tumour microenvironment, such as hypoxia, oxidative stress and ER stress. This 
includes Glucose-Regulated Protein 78 (GRP78), a stress-responsive protein found within the ER lumen. It functions as an ER chaperone (also known as binding immunoglobulin protein (BiP) or heat shock $70 \mathrm{kDa}$ protein 5 (HSPA5)) and translocate proteins from the translocon to the ER lumen for glycosylation (Serrano-Negrón et al., 2018). This also includes NFE2L1 (also known as Nrf1) and NFE2L2 (also known as Nrf2); two principal CNC-bZIP proteins with similar, but different, structural domains that are involved in the response to oxidative stress (Zhang et al., 2009; Zhang and Hayes, 2010). NFE2L2 has been generally accepted as a master regulator of Antioxidant Response Element (ARE) controlled gene expression ( $\mathrm{Li}$ et al., 2019). Hypoxia-inducible factors (HIFs) are $\mathrm{O}_{2}$ regulated transcriptional factors that play critical roles in low-oxygen (hypoxia) adaptive mechanisms. Increased levels of HIFs have been identified in many solid tumors, such as brain, breast, cervical, gastrointestinal, lung, oropharyngeal, and ovarian cancers (Semenza et al, 2003, 2007, and 2010). Tumour hypoxia has been associated with the WNT signaling pathway in the modulation of cancer progression (Logan and Nusse, 2004).

In this study we examined the role of stressors including hypoxia, ER, and oxidative stress in activating GRP78, HIF1 $\alpha$, NFE2L1, and NFE2L2 in HIEC-6 cells. In previous work, we examined the role of stressors on PORCN, WLS and $\beta$-catenin expression and we established that ER stress regulates the secretion of WNT proteins. In addition, we assessed the importance of SCD1 desaturation of palmitic acid and the attachment of lipids (both palmitoleation for PORCN and palmitoylation for WNTs) in activating GRP78, HIF1 $\alpha$, NFE2L1, NFE2L2, WNT3A, WNT5A, PORCN, and WLS. 


\subsection{Results}

\subsubsection{Effect of SCD1 and 2BHDA on the cell viability}

Experimental investigation of the growth suppression effects of SCD1 and 2BHDA were assessed on cell vialbility using MTT assay. The cytotoxicity induced by 2 BHDA and SCD1 inhibitor treatments on HIEC-6 cell was assessed by MTT assay. HIEC-6 cells were exposed to increasing concentrations of 2BHDA or SCD1 inhibitor for $24 \mathrm{~h}$. In HIEC6 cells viability decreased with an increase in 2BHDA and SCD1 inhibitor concentrations (Fig. 4.1a and b). The HIEC-6 revealed that $100 \mu \mathrm{M}$ and $10 \mu \mathrm{M}$ were the optimal concentrations for cell treatment for 2BHDA and SCD1 inhibitor respectively. However, beyond these optimal concentrations, cells became aggregated hence cell death occurred. 


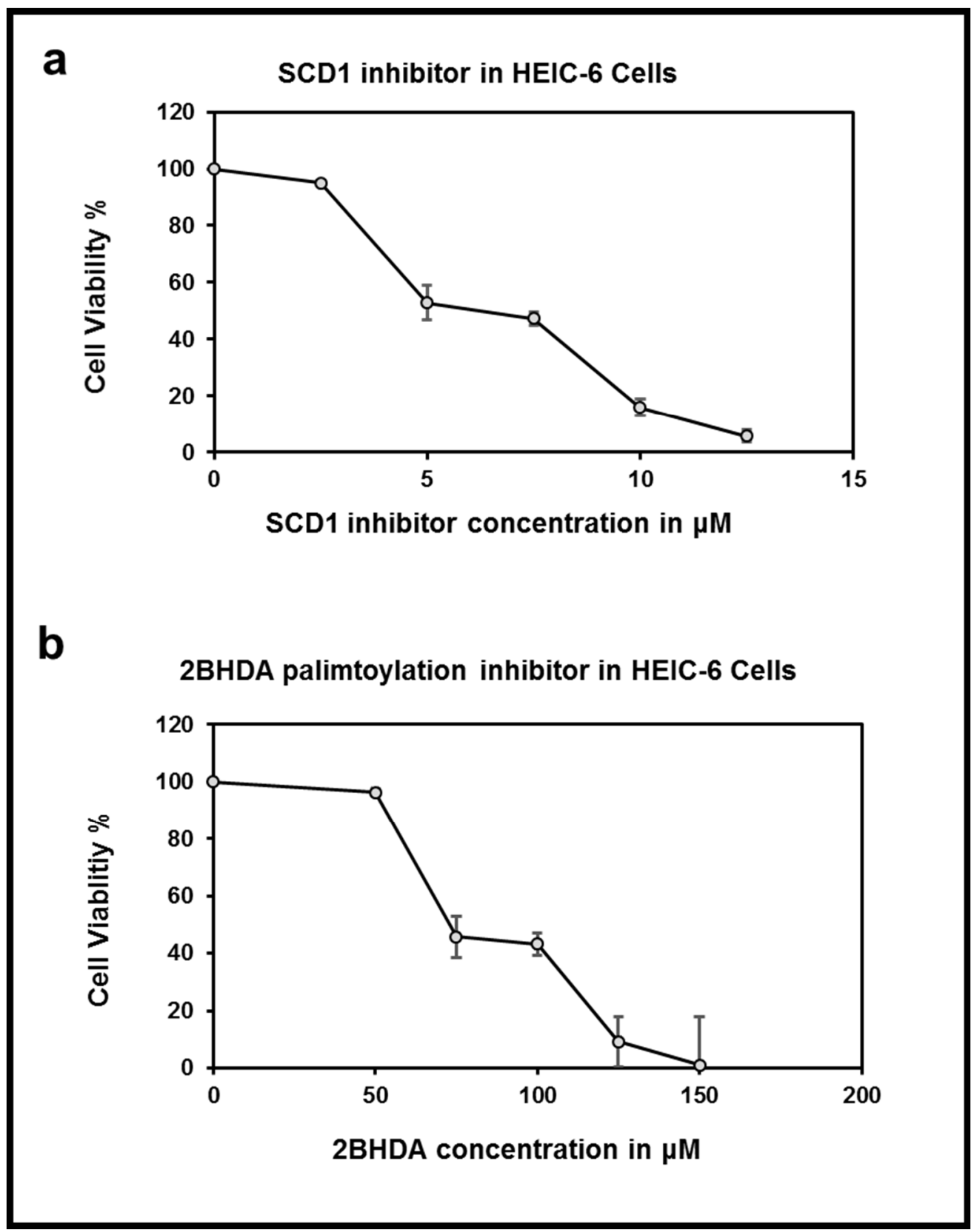

Figure 4.1: Cell viability assay. The viability of HIEC-6 cells, in exponential stage of growth, after $24 \mathrm{hrs}$ of exposure to a) SC1 inhibitor and b) 2BHDA as measured by the MTT (3-(4,5-dimethylthiazol-2-yl)-2,5-diphenyltetrazolium bromide) assay. Results are expressed as means \pm S.E.M of three independent experiments. 


\subsubsection{Gene expression of transcription factors when treated with SCD1 inhibitor and inhibition of palmitoyleation}

The gene expression of the four stress-related transcription factors were investigated by testing their potential to be inhibited by SCD1 or 2BHDA inhibitors. qPCR was used to evaluate the level of the gene expression in HIEC-6 cells. The level of gene expression of

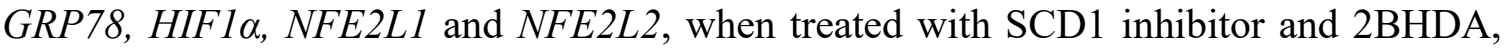
were all decreased (Fig. 4.2a, b, and d) with exception of the NFE2L1 gene which was only reduced by SCD1 inhibitor (Fig. 4.2c). 


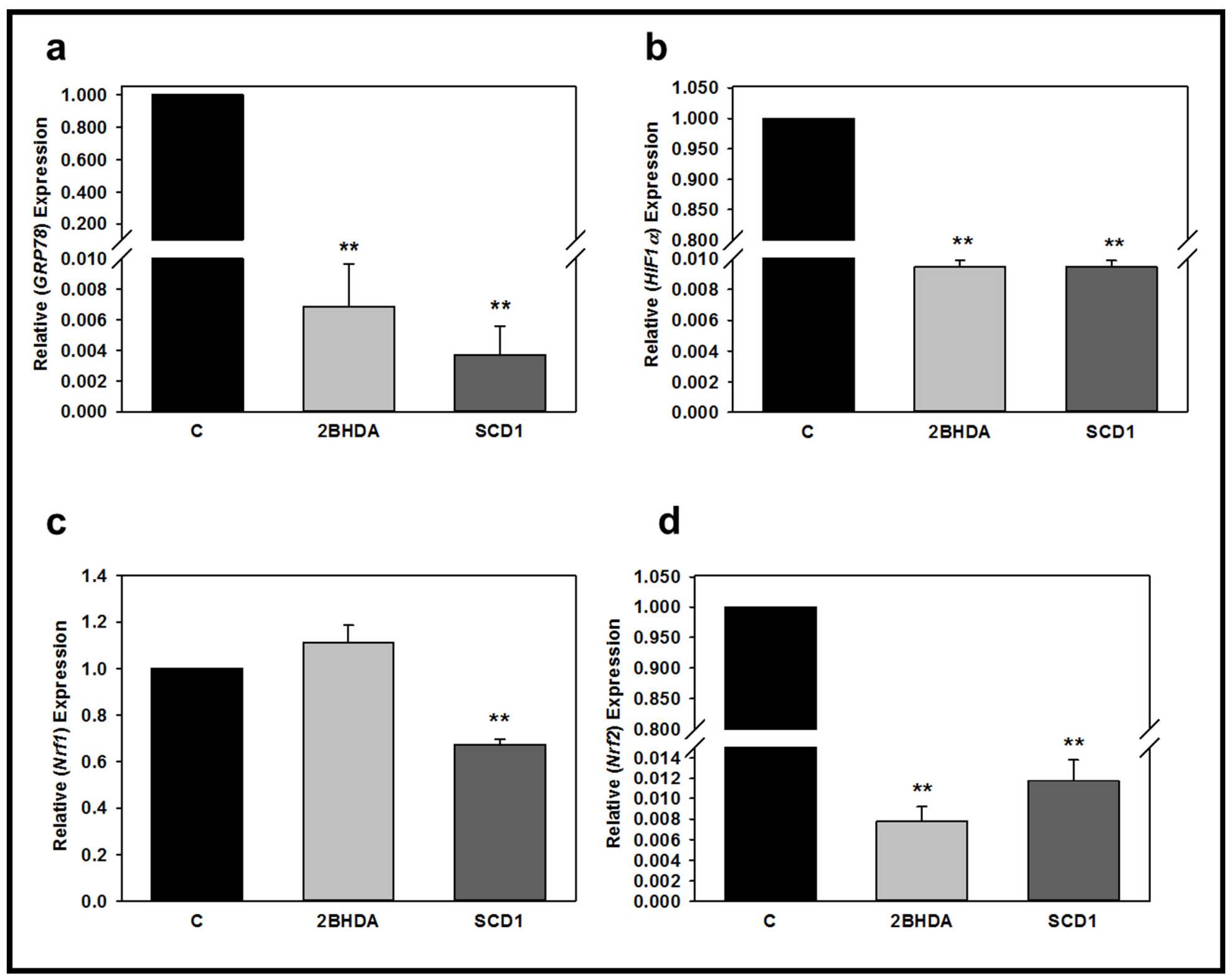


Figure 4.2: The effect of 2BHDA and SCD1 inhibitor on GRP78, HIF1 $\alpha$, NFE2L1 and NFE2L2 gene expression in human intestinal epithelium cell 6 (HIEC-6) cells. HIEC-6 cells were treated with $100 \mu \mathrm{M}$ of 2BHDA and $10 \mu \mathrm{M}$ of SCD1 inhibitor (24 hrs). Cells without treatment were used as controls. Total RNA was isolated from HCT116 cells, converted to cDNA and analyzed by qPCR using gene-specific primers for a) GRP78, b) HIF1 $\alpha$, c) NFE2L1 and d) NFE2L2 and target genes. The level of target genes of each mRNA was normalized to the GAPDH mRNA. Data show the means from three independent experiments \pm S.E.M. Data analysis was done using one-way ANOVA followed by Dunnett's test; *p $<0.05, * * \mathrm{p}<0.01, * * * \mathrm{p}<0.001$. 


\subsubsection{Gene expression of WNT-associated genes with 2BHDA and SCD1 inhibitor treatments}

Gene expression of four primers were investigated by testing their potential to be inhibited by SCD1 or 2BHDA chemical inhibitors. qPCR was used to evaluate the level of the expression in HIEC-6 cells. The level of PORCN, WLS, and WNT3A mRNA was decreased when cells were treated with SCD1 inhibitor and 2BHDA (Figures 4.3a, $\mathrm{b}$ and c). However, WNT5A was only inhibited by SCD1 inhibitor (Fig. 4.3d). 


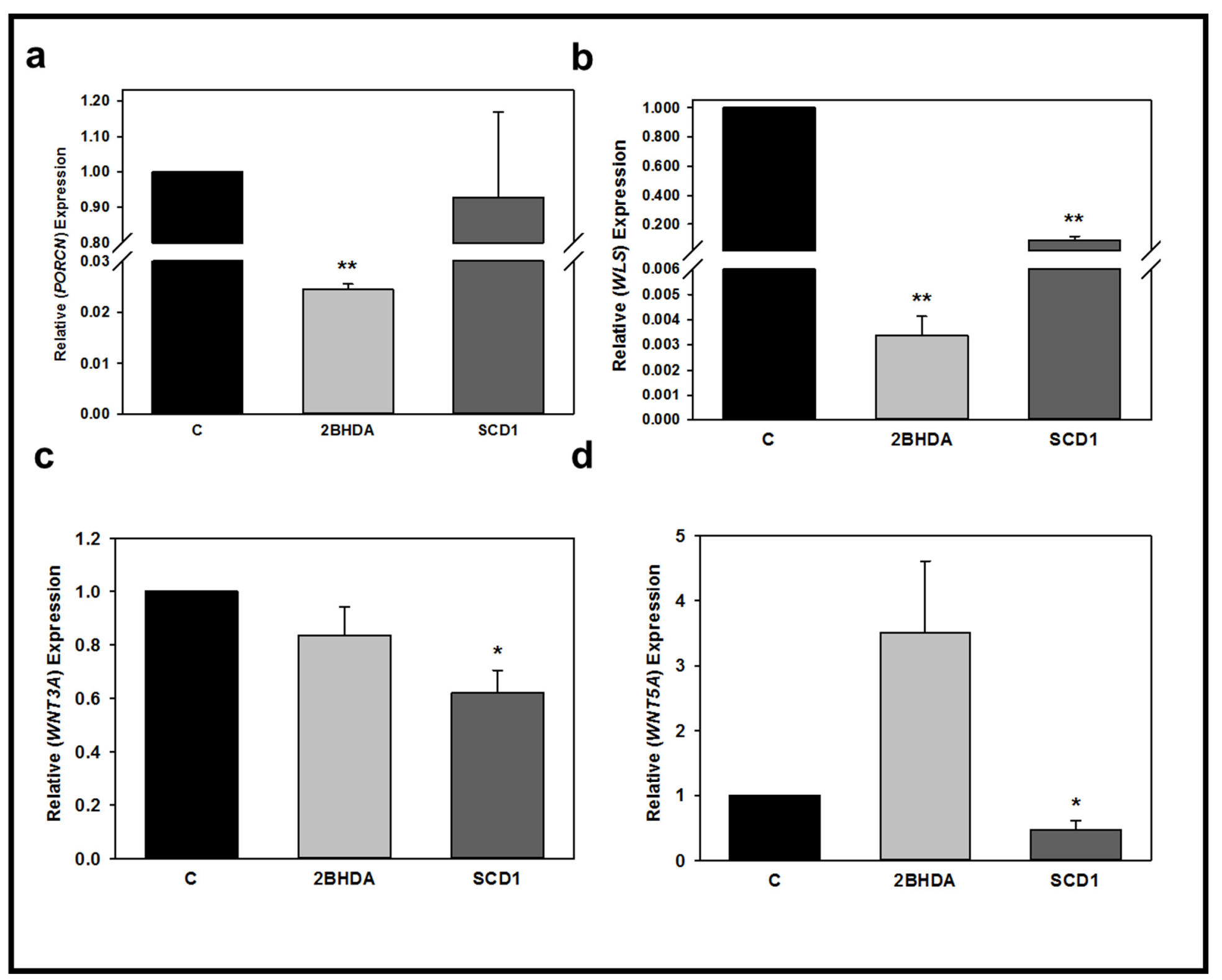


Figure 4.3: The effect of 2BHDA and SCD1 inhibitor on PORCN, WLS1 $\alpha, W N T 3 A$ and $W N T 5 A$ gene expression in human intestinal epithelium cell 6 (HIEC-6) cells. HIEC-6 cells were treated with $100 \mu \mathrm{M}$ of 2BHDA and $10 \mu \mathrm{M}$ of SCD1 inhibitor (24 hrs). Cells without treatment were used as controls. Total RNA was isolated from HCT116 cells, converted to cDNA and analyzed by qPCR using gene-specific primers for a) PORCN, b) WLS, c) WNT3A and d) WNT5A and target genes. The level of target genes of each mRNA was normalized to the GAPDH mRNA. Data show the means from three independent experiments \pm S.E.M. Data analysis was done using one-way ANOVA followed by Dunnett's test; *p $<0.05, * * \mathrm{p}<0.01,{ }^{* * *} \mathrm{p}<0.001$. 


\subsubsection{Expression profile of GRP78, HIF1a, NFE2L1 and NFE2L2 genes when treated with hypoxia, ER and oxidative stress.}

The expression profile of the GRP78, HIF $1 \alpha, N F E 2 L 1$ and NFE2L2 genes were measured using, qPCR as well as treated HIEC-6 cells to study the difference in their transcription level in hypoxia, ER and oxidative stress treatments compared to that of the control. HIEC-6 cells showed a significant upregulation of GRP78, HIF 1 $\alpha$, NFE2L1 and NFE2L2 gene expression when treated with hypoxia, ER and oxidative stresses (Fig. 4.4a, c, and d) with the exception of HIF $1 \alpha$ which exhibited downregulation when treated with DFO and Thap (Fig. 4.4b). 


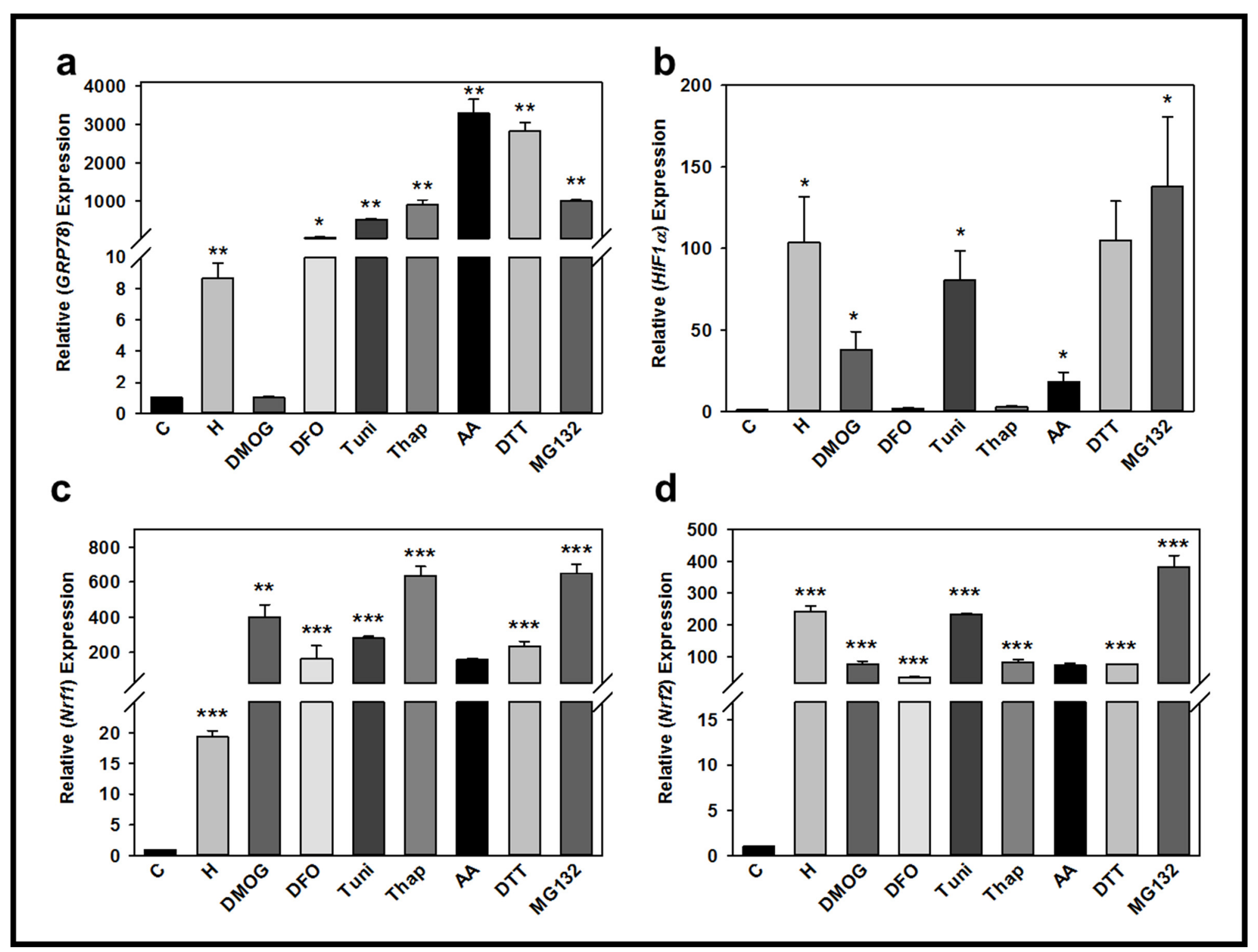


Figure 4.4: The effect of hypoxia and hypoxic mimetics, ER stressors and oxidative stressors on GRP78, HIF1 $\alpha$, NFE2L1 and NFE2L2 gene expression in Human Intestinal Epithelium Cells (HIEC-6). HIEC-6 cells were treated with hypoxia $\left(1 \% \mathrm{O}_{2}\right.$ and $\left.5 \% \mathrm{CO}_{2}\right)$ and hypoxic mimetics (100 $\mu \mathrm{M}$ desferrioxamine (DFO) and $1 \mathrm{mM}$ dimethyloxalylglycine (DMOG)), (5 mg/mL tunicamycin (Tuni), $3 \mathrm{nM}$ thapsigargin (Thap), (90 mM antimycin A (AA), $2 \mathrm{M}$ dithiothreitol (DTT)) for $24 \mathrm{hr}$ and $5 \mathrm{mg} / \mathrm{mL}$ MG132 for $3.5 \mathrm{hrs}$. Cells without treatment were used as controls. Total RNA was isolated from HIEC-6 cells, converted to cDNA and analyzed by qPCR using genespecific primers for a) GRP78, b) HIF1 $\alpha$, c) NFE2L1 and d) NFE2L2 and target genes. The level of target genes of each mRNA was normalized to the GAPDH mRNA. Data show the means from three independent experiments \pm S.E.M. Data analysis was done using one-way ANOVA followed by Dunnett's test; *p $<0.05, * * \mathrm{p}<0.01, * * * \mathrm{p}<0.001$ 


\subsubsection{Relative $\beta$-catenin expression with overexpression of PORCN, WLS and WNT3A}

To assess the relationship between the secretion of WNT proteins and $\beta$-catenin, PORCN, WLS, and WNT3A were overexpressed in HIEC-6 and HCT116 cells and cells were treated with MG132 using Western blotting. $\beta$-catenin levels were elevated when overexpressed and treated with MG132 in both HIEC-6 and HCT116 cells (Fig. 4.5a and b).

$\beta$-catenin levels were higher in the overexpressed WLS, WNT3A, and PORCN respectively (Fig. 4.5a and b). The gene expression of $\beta$-catenin was similar in both HCT116 and HIEC-6 cells. 


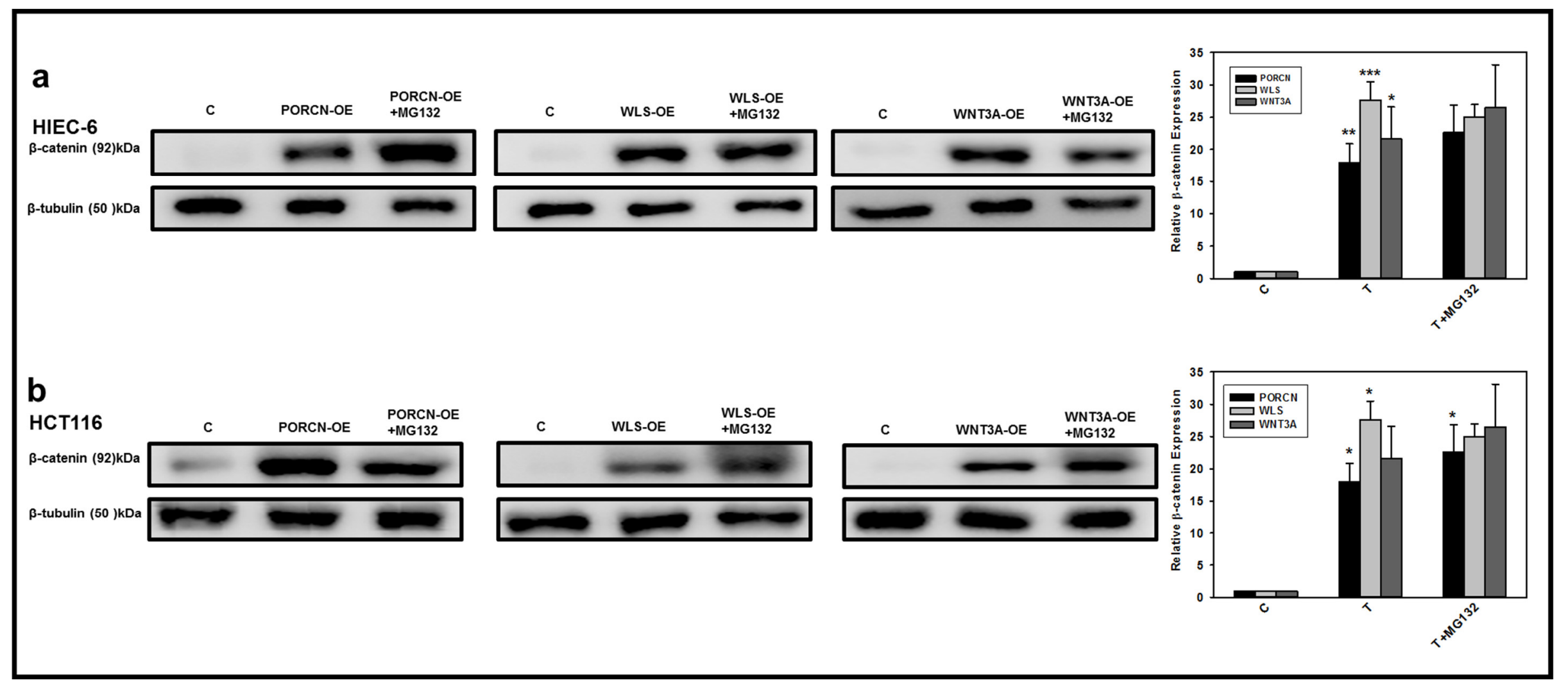


Figure 4.5: PORCN, WLS, and WNT3A proteins were overexpressed (OE) using Oligofectamine in (a) HIEC-6 and (b) HCT116 cells and proteasome inhibitor (MG132) was used to treat cells overexpressing PORCN, WLS and WNT3A. The Western blot analysis showed that overexpression of PORCN, WLS and WNT3A-OE increased $\beta$-catenin protein expression. The level of protein expression of each sample was normalized to the expression of $\beta$-tubulin. Data are the mean band densities from three independent experiments \pm S.E.M. Data analysis was done using one-way ANOVA followed by Dunnett's test; ${ }^{*} \mathrm{p}<0.05,{ }^{* *} \mathrm{p}<0.01,{ }^{* * *} \mathrm{p}<0.001$. 


\subsubsection{Protein expression of transcription factors}

The GRP78, HIF1 $\alpha$, NFE2L1 and NFE2L2 showed upregulation when overexpressed and treated with MG132 in HCT116 cells. Conversely, all genes overexpressed and treated with MG132 showed a higher expression in GRP78, HIF1 $\alpha$, NFE2L1 and NFE2L2 (Fig. 4.6a, b, $d$ and e), with the exception GRP78 with OE-WNT3A, NFE2L2 with OE-PORCN, OEWLS, and OE-WNT3A) (Fig. 4.7c and f). The expression of GRP78 with OE-WNT3A was the lowest in HIEC-6 cells. However, HIF1 $\alpha$, NFE2L1 and NFE2L2 levels were elevated in all the overexpressed and treated in both PORCN and WLS in HIEC-cells (Fig. 4.7a, b, c, d, e and f). The overexpressions of all the transcription factors were higher in HCT116 cells, compared to HIEC-6 cells. Moreover, the overexpression of PORCN and WLS in cells resulted in a much higher expression of all the transcription factors in both HIEC-6 and HCT116 cells. 


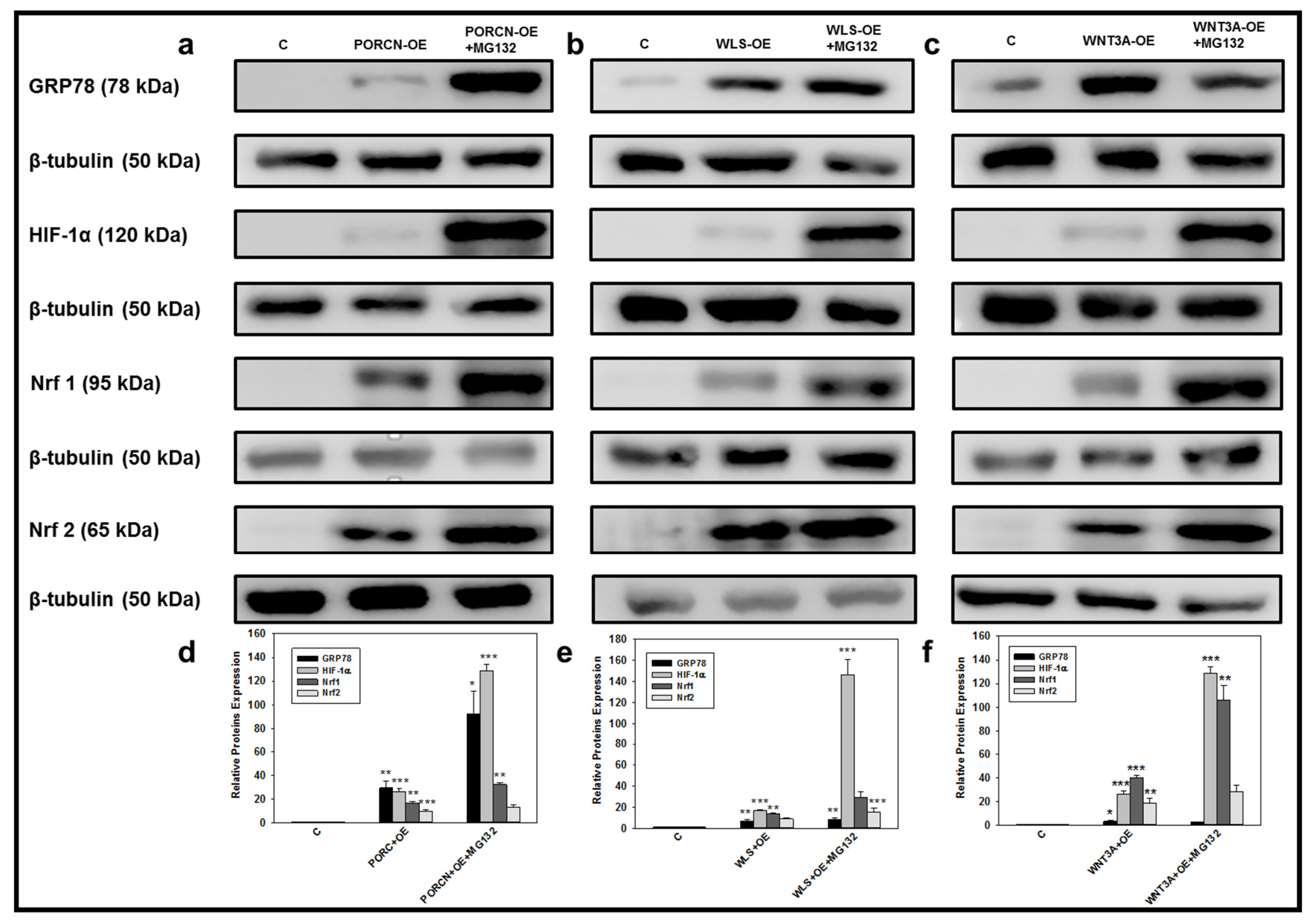


Figure 4.6: PORCN, WLS, and WNT3A were overexpressed (OE) using Oligofectamine, in human intestinal epithelial cells (HIEC-6) and treated with proteasome inhibitor (MG132). a) The Western blot analysis showed that PORCN, WLS WNT3A-OE promoted the levels of GRP78, HIF1 $\alpha$, NFE2L1, and NFE2L2 related protein expression. b) Figure (b), (c) and (d) corresponding densitometric quantification profile represents the measurement of protein expression. The level of protein expression of each sample was normalized to $\beta$-tubulin. The data shown is the mean band densities from three independent experiments \pm S.E.M. Data analysis was done using one-way ANOVA followed by Dunnett's test; *p $<0.05, * * \mathrm{p}<0.01,{ }^{* * *} \mathrm{p}<0.001$. 


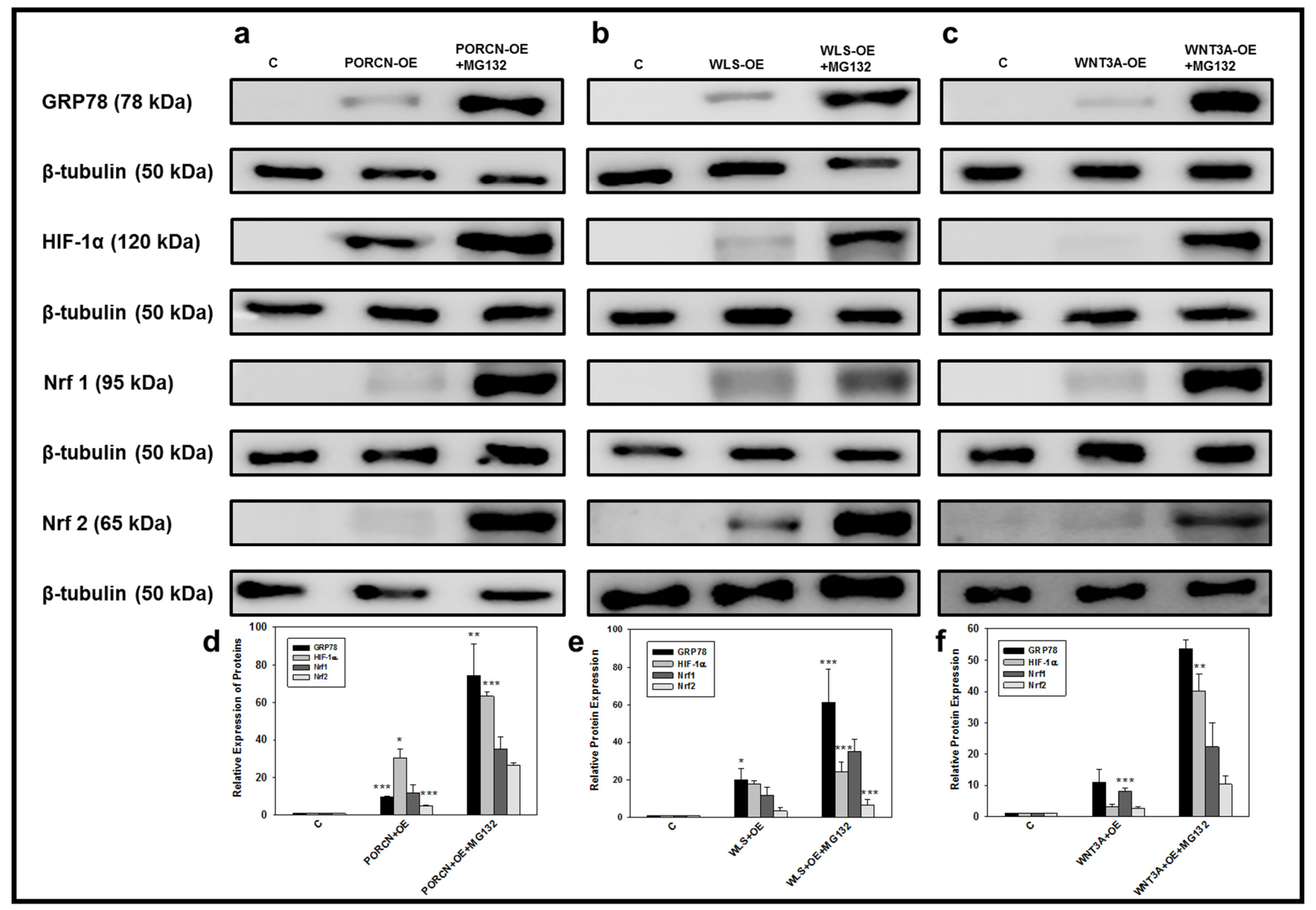


Figure 4.7: PORCN, WLS, and WNT3A were overexpressed (OE) using Oligofectamine in colon cancer adenocarcinoma cell (HCT116) cells and treated with proteasome inhibitor (MG132) . a) The Western blot analysis showed that PORCN, WLS WNT3A induced increased levels of GRP78, HIF1 $\alpha$, NFE2L1, and NFE2L2 protein expression. b) Figures (b), (c) and (d) corresponding densitometric quantification profile represent the measurement of protein expression. The level of protein expression of each sample was normalized to $\beta$-tubulin. The data shown is the mean densities from three independent experiments \pm S.E.M. Data analysis was done using one-way ANOVA followed by Dunnett's test; *p $<0.05, * * \mathrm{p}<0.01,{ }^{* * *} \mathrm{p}<0.001$. 


\subsection{Discussion}

\subsubsection{Palmitoyleations and stearoyl CoA desaturases genes regulates GRP78, HIF1a,}

NFE2L1 and NFE2L2

Stearoyl-CoA desaturase 1 (SCD1) is a central enzyme in lipid metabolism that catalyzes the $\Delta 9$-desaturation of the saturated fatty acids palmitate and stearate to the monounsaturated fatty acids (MUFA) palmitoleate and oleate, respectively (Ntambi and Miyazaki, 2003). In the absence of SCD1 activity, endogenous MUFA production is decreased, resulting in reduced lipid accumulation due to decreased fatty acid synthesis and increased fatty acid oxidation (Miyazaki, et al., 2006). In our study, we investigated the relationship between WNT secretion proteins and SCD1 to the integrated stress response. The MTT assay was used to assess the cytotoxicity of SCD1 inhibitor and 2BHDA treatments on HIEC-6 cells. Our data from this assay demonstrated a clear concentration-response regarding the cytotoxicity of SCD1 inhibitor and 2BHDA treatments at 5 and $100 \mu \mathrm{M}$, respectively, in HIEC-6 cells. We chose GRP78, HIF $1 \alpha$, NFE2L1 and NFE2L2 common transcription factors that are involved in common stress responses (ER stress, hypoxia, oxidative stress) (Wang et al., 2009). GRP78 binds to misfolded proteins and unassembled complexes and initiates ER-associated degradation (ERAD) which is responsible for UPR regulation. Activation of the UPR decrease the translation of proteins within the secretory pathway and enhances their correct folding (Ni et al., 2011). A higher level of transcription factors contributes to the acquisition of phenotypic cancer hallmarks including apoptosis resistance, metastasis, and angiogenesis (Li and Li, 2012). The expression of GRP78, HIF1 $\alpha, N F E 2 L 1$, and NFE2L2 mRNA levels were examined under conditions where the palmitoylation and palmitoleation of secretory 
proteins, was inhibited in HIEC-6 cells. Palmitate (palmitic acid) cannot be used by PORCN as a substrate for modifying target proteins and must be first desaturated to palmitoleate (palmitoleic acid) prior to their linkage to proteins. 2-Bromohexadecanoic acid (2BHDA) is a metabolically stable analog of the fatty acid palmitic acid and is a known inhibitor of PPAR $\delta$ (peroxisome proliferator-activated receptor, delta isoform). It has been shown to inhibit fatty acid oxidation, palmitoylation of target proteins, and glucose uptake by cells. Inside the cell the 2BHDA is converted to 2BHDA-CoA, the binding of 2BHDACoA to a palmitoyl acyltransferase (PAT), resulting in an inhibitor: enzyme complex (Resh, 2006). Additionally, 2BHDA is transferred to the target protein, but the decreased hydrophobicity compared with palmitate which reduces the binding of the protein to the lipid bilayer (Resh, 2006). Furthermore, 2BHDA may alter lipid metabolism by reducing the levels of intracellular palmitoyl-CoA that are available for palmitoylation. In fact, this compound inhibits a number of enzymes involved in lipid metabolism including fatty acid CoA ligase and glycerol-3-phosphate acyltransferase, as well as triacylglycerol biosynthesis (Chase and Tubbs, 1972; Coleman et al., 1992). As shown in Fig. 4.2a-d, the GRP78, HIF1 $\alpha, N F E 2 L 1$, and NFE2L2 mRNA levels decreased after the treatments with 2BHDA and SCD1 inhibitors, suggesting that the regulation of GRP78, HIF1 $\alpha$, NFE2L1, and NFE2L2 by stearoyl CoA desaturase and palmitoylation synthesis stimulation occurs at the post-transcriptional level. SCD1 and palmitoylation processes are key molecular factors responsible for WNT biogenesis and processing and their inhibition provides an alternative mechanism for blocking WNT pathway activation and key transcription factors that are responsible for the proper folding of proteins. 


\subsubsection{Stearoyl CoA desaturase is required to produce active, lipid-modified WNT proteins}

In the current study, we showed that stearoyl CoA desaturase (SCD1) and palmitoylation are essential for regulating PORCN, WLS, WNT3A and WNT5A gene expression (Fig. 4.3a, b, c and d). Palmitoyleation is modulated by nutrient-dependent signals which impact the regulation of gene expression (Spinelli et al., 2018). Moreover, we speculate that 2BHDA and SCD1 inhibitors induce the ER-stress-related gene and protein expressions (Fig. 4.3a, b, c, and d). These genes could be directly or indirectly related to GRP78, HIF1 $\alpha, N F E 2 L 1$, and NFE2L2 (Fig. 4.2a-d). Previous studies showed that treated cells with SCD1 inhibitors blocked incorporation of palmitate analogs into $W N T 3 A$ and $W N T 5 A$, and reduced WNT secretion as well as WNT signaling (Rios-Esteves and Resh, 2013). Similarly, our results showed a decline in the level of gene expression of PORCN, WLS, WNT3A, and WNT5A when treated with SCD1 inhibitors and 2BHDA, with the exception of WNT5A only inhibited by SCD1. Interestingly, PORCN and WLS gene expression was significantly reduced when treated with 2BHDA, as compared to SCD1 inhibitor (Fig. 4.3a and b). However, SCD1 inhibitor reduced the expression of WNT3A and WNT5A, compared to 2BHDA. A previous study indicated palmitoylation plays a crucial role in transmembrane domain arrangement of the co-receptor protein LRP6 which binds to Fz (Abrami et al., 2008). SCD1 function is an essential preliminary step for palmitoleation of target proteins by PORCN (Fig. 4.3c). However, WNT5A showed an increase in the gene expression with the treatment of 2BHDA (Figs. 4.3c and d). 


\subsubsection{Treatment with ER, hypoxia and oxidative stresses elevate the gene expression of GRP78, HIF1 alpha, NFE2L1 and NFE2L2 in HIEC-6 cells}

It's known that GRP78, HIF1 $\alpha, N F E 2 L 1$, and NFE2L2 are regulated under stressors (Lee et al., 2017). Previous studies showed that stress conditions promote cell survival, cell proliferation and angiogenesis (Hubbi and Semenza, 2015). Treatments with hypoxia and hypoxia mimics, Tuni, Thap, AA, DTT, and MG132 showed elevated gene expression in all transcription factor gene expression (GRP78, HIF1 $\alpha, N F E 2 L 1$, and NFE2L2). This is not surprising as these genes are highly regulated under stress (Fig. 4.4a, b, c and d). Several transcription factors have been involved in the regulation of gene expression under hypoxia and oxidative stress conditions (Biswas and Chan, 2010). We focused on a few

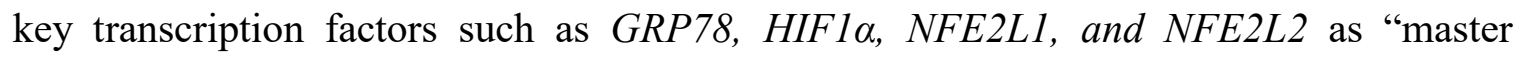
regulators" of these specific stress responses. These regulatory transcription factors control the expression of the majority of the genes involved in ER stress, hypoxia, and oxidative stress respectively (Hybertson et al., 2011). GRP78/BiP plays a crucial role in the ER stress response by deactivating ER receptors (PERK, IRE1, and ATF6). These three proteins activate three separate ER stress-related pathways. Once unfolded proteins begin to accumulate in the ER lumen, GRP78/BiP is removed from these three proteins, activating their function in the UPR. Our results showed a strong evidence that these transcription factor activities, under their respective stresses, are linked to the activation of the WNT proteins (Fig. 4.5a and b). Our assumptions were that transcription factors such as NFE2L1 and NFE2L2 function as dominant tumour suppressors by intrinsic inhibition of the WNT/ $\beta$-catenin pathways, and other signaling networks involved such as cancer metabolic pathways. Therefore, the aberrant activation of this $\mathrm{WNT} / \beta$-catenin signaling by 
impairment of the core proteasome subunits is much more likely to play a pivotal role in activating oncongenic genes. Moreover, the elevated gene expression for these transcription factors could be related to impaired mitochondria (or mitochondrial dysfunction) which increases ER stress proteins such as GRP78, HIF1 $\alpha, N F E 2 L 1$, and NFE2L2.

\subsubsection{Overexpression of PORCN, WLS, and WNT3A induces levels of GRP78,}

\section{HIF1 $\alpha$, NFE2L1, and NFE2L2 and $\beta$-catenin protein expression}

To further elucidate the mechanism underlying the upregulation of transcription factors and WNT proteins, the proteasome inhibitor MG-132 was used. As shown in Fig. 4.3, PORCN, WLS, and WNT3A overexpression combined with MG132 treatment, had an effect on the GRP78, HIF1 $\alpha$, NFE2L1, NFE2L2 and $\beta$-catenin proteins in HCT116 and HIEC-6 cells. Together, the data indicate that the upregulation of GRP78, HIF1 $\alpha$, NFE2L1, NFE2L2, and $\beta$-catenin proteins by MG132 treatment, or PORCN, WLS and WNT3A overexpression, is dependent on degradation of proteins by the proteasome. From our data, ER stressors could be controlling the secretion of the WNT proteins. Deregulation of WNT/ $\beta$-catenin pathway usually occurs in colorectal cancer, which leads to a constitutively stable and active $\beta$-catenin and induces aberrant cell proliferation (Vermeulen et al., 2010). $\beta$-catenin promotes epithelial architecture by forming cell surface complexes with Ecadherin and also interacts with TCF/LEF-1 in the nucleus to control gene expression. By DNA transfection, we overexpressed $\beta$-catenin in HIEC-6 and HCT116 cells. Our present data indicated that $\beta$-catenin was elevated when cells were overexpressed with PORCN, WLS, and WNT3A (Figs. 4.7 and 4.8 a-f). Thus, $\beta$-catenin not only regulates cell growth 
and proliferation but also supports cell survival under the unfavorable conditions encountered in the tumour microenvironment.

\subsection{Conclusions}

In summary, the effects of stress on WNT signaling pathways may be through key transcription factors known to be induced by stress (HIF by hypoxia, GRP78by ER stress, and NFE2L1 and NFE2L2 by oxidative stress). Other co-activators and transcription factors involved in ER stress, such as ATF6, XBP1 and ER stress-associated pro-apoptotic factors, may also influence WLS and PORCN expression. Deregulation of transcription factors is a frequent occurrence in colon cancer, and the accompanying drastic changes in gene expression profiles play fundamental roles in multistep process of tumorigenesis; from cellular transformation to disease progression to metastatic disease (Xu et al., 2020). These stress-inducible factors are known to play key roles in the development of colon cancer. These findings establish a role for SCD1 as an essential intermediate in WNT protein biogenesis and processing and reveal SCD1 inhibition as an alternative mechanism for blocking WNT pathway activation. Understanding how WLS and PORCN gene and protein expressions are altered under these stress conditions will provide insight into how their functions are altered in the tumour microenvironment and how this, in turn, affects the secretion of the WNT proteins. Future studies will focus on the role of stress-inducible factors in WNT signaling and its involvement in cell proliferation and differentiation. 


\section{Chapter 5: Cadmium induced toxicity on WNT proteins and transcription factors}

\subsection{Introduction}

Cadmium (Cd) is a transition metal and toxic pollutant, constituting a major occupational and environmental hazard, and has a biological half-life of 10-30 years (Staessen, et 1., 1999; Schutte et al., 2008). It is released into the environment through natural processes and human activities, such as mining and smelting, which may lead to contamination of soils and to increased cadmium uptake by crops and vegetables that are grown for human consumption ( $\mathrm{Lv}$ et al., 2017). The environmental prevalence of cadmium is a major concern because of its high rates of soil-to-plant transfer, cadmium is a contaminant found in most human foodstuffs, which renders diet a primary source of exposure among nonsmoking, nonoccupationally exposed populations (Clemens, 2006; Franz et al., 2008). Further, accumulating evidence links environmental exposure to cadmium with increased cancer incidence globaly, it was determined excess cancer mortality was associated with environmental exposure to cadmium (Akesson et al., 2008; Menke et al., 2009). Cadmium is a known as a nephrotoxin as it is highly damaging to kidney. Ingested cadmium binds to metallothioneins, cysteine-rich proteins forming $(\mathrm{Cd}-$ MT complex) in the liver (Klaassen, et al., 2009). Metallothionein 1 (MT1) is degraded in the endosomes and the released free cadmium in the cytosol generates 'Reactive Oxygen Species' (ROS), causing oxidative damage (Johri et al., 2010). DNA modifications are known to occur due to cadmium-induced oxidative damage (Fernandez, et al., 2003). All these cellular effects induced by cadmium are often associated with apoptosis, induced by 
p53 signaling (Fernandez, et al., 2003). Adverse health effects, including bone damage and nephrotoxicity, occur with long-term exposure to water and food contaminated with cadmium. Cadmium gets absorbed in the gut and only about $5 \%$ of the ingested cadmium is absorbed through the gastrointestinal tract and majority of cadmium gets metabolized in the liver and kiney and eliminated by urine (Friberg et al., 1985). Cadmium is a severe pulmonary and gastrointestinal irritant, which can be fatal if inhaled or ingested. After acute ingestion, symptoms such as abdominal pain, burning sensation, nausea, vomiting, salivation, muscle cramps, vertigo, shock, loss of consciousness and convulsions usually appear within 15 to $30 \mathrm{~min}$ (Baslet and Carvey, 1995). Cd contributes to ER stress, and the eukaryotic initiation factor $2 \alpha$-subunit (eIF2 $\alpha$ )-activating transcription factor 4 (ATF4) pathway is involved in cellular adaptation to cadmium (Harding et al., 2008). Furthermore, cadmium is considered a Class I carcinogen and disrupts the E-cadherin/ $\beta$-catenin complex of epithelial adherent junctions, resulting in renal cancer (Pearson and Prozialeck, 2001). Deregulation of E-cadherin adhesion and changes in WNT/ $\beta$-catenin signaling are known to contribute to carcinogenesis (Pearson and Prozialeck, 2001). The molecular and genetic basis by which $\mathrm{CdCl}_{2}$ causes disease remains largely unknown.

The WNT/B-catenin pathways promote embryonic development, differentiation, proliferation, and cell renewal (Valvezan, et al., 2012). Porcupine (PORCN) and WNTless (WLS) are factors that control the production of WNT; a protein involved in the early stages of gastric cancer development (Pearson and Prozialeck, 2001). Initiation of WNT signaling occurs through disassociation of the negative regulating complex GSK-3/APC (glycogen synthase kinase 3/adenomatosis polyposis coli), allowing for activation of $\beta$ - 
catenin (Valvezan, et al., 2012). It is known that cadmium causes lesion in the gastric lining, resulting in the development of gastric cancer, (Pearson and Prozialeck, 2001).

Cadmium could potentially affect WNT signalling through the induction of reactive oxygen species, resulting in the upregulation of gene and protein expression. Furthermore, in the cell $\beta$-catenin has as a structural protein in adherens-junctions, participating in cellcell adhesion by bridging E-cadherin to $\alpha$-catenin. The cadherins are $\mathrm{Ca}^{2+}$-dependent cell adhesion glycoproteins that physically link neighboring cells together (Shapiro and Weis, 2009). Cadmium is known to disrupt the cell adhesion and displace $\mathrm{Ca}^{2+}$ leading disruption of E-cadherin interaction (Danilkovitch-Miagkova et al., 2009). In addition, other studies showed an increased expression of WNT target genes could contribute to increased proliferation, survival as well as decreased cell death of which is exposed to $\mathrm{Cd}^{2+}$ but also other forms of stress-induced damage and thereby promote $\mathrm{Cd}^{2+}$ carcinogenesis (Méplan et al., 1999).

In this study, we aimed to examine the role of cadmium exposure on the WNT signalling pathway and determine the activation of different transcription factors are activated due to the cadmium exposure. To reveal the effects of cadmium exposure on transcription we have chosen a set of factors that are the "master regulators" of various stress responses; 78-kDa glucose-regulated protein (GRP78) regulates gene expression in response to ER stress, hypoxia inducible factor 1 alpha (HIF1 $\alpha)$ regulates gene expression involved in hypoxic stress and nuclear factor erythroid-2-like 1 and 2 (NFE2L1 and NFE2L2 or Nrf1 and Nrf2 respectively) regulate gene expression in response to oxidative stress. NFE2L1 and NFE2L2 are members of the vertebrate Cap'n'Collar (CNC) transcription factor family that commonly contains a unique basic-leucine zipper domain. 
Among CNC family members, NFE2L2 is known to regulate a battery of antioxidant and xenobiotic-metabolizing enzyme genes through the antioxidant response element (ARE); a consensus DNA sequence found in the enhancers of genes that respond to oxidative and toxin stresses. The gene for metallothionein 1 is controlled by an ARE that is more specific to NFE2L1 than NFE2L2. Metallothioneins are ubiquitous, low molecular weight cysteinerich proteins that bind to heavy metals to detoxify them (Margoshes and Vallee, 1957). In addition, in this study, we have tried to evaluate the difference in the degree of cytotoxicity induced by the heavy metal cadmium on HCT116 and HIEC-6 cells. It is little known about the impact of molecular mechanism of cadmium on WNT signalling pathway. Therefore, we explored the cellular and molecular mechanisms induced by exposure to different concentrations of cadmium chloride $\left(\mathrm{CdCl}_{2}\right)$, on two different human colon cell lines; human intestinal epithelium cells (HIEC-6) and human colorectal cancer (HCT116) cells. We tested the effect of exposure to cadmium on WNT and WNT-associated factors gene and protein expression. This includes Porcupine (PORCN), WNTless (WLS), the canonical pathway WNT (WNT3A) and the non-canonical pathway WNT (WNT5A). Furthermore, we tested various transcriptional regulators in response to stress including GRP78, HIF1 $\alpha$, MT1, NFE2L1, and NFE2L2. 


\subsection{Results}

\subsubsection{Effect of cadmium chloride on cell viability}

The cytotoxicity induced by $\mathrm{CdCl}_{2}$ on two cell line lines, HCT116 and HIEC-6, was assessed using MTT assay. HCT116 and HIEC-6 cell lines were exposed to increasing concentrations of cadmium chloride for $24 \mathrm{hrs}$. Cadmium had a dose-dependent effect on viability of all the cell lines in this work (Fig. 5.1) in the two cell lines. The cell viability was reduced with increase in cadmium concentration. Data obtain from MTT assay demonstrated a strong concentration-response relation with regard to the cytotoxic cadmium chloride in HCT116 and HIEC-6 cells. In the present study, we tested cadmium chloride concentrations of $0,2.5,5,7.5 .10,12.5$ and $15 \mu \mathrm{M}$. Concentrations of cadmium ranging from $12.5-15 \mu \mathrm{M}$ showed a significant reduction in cell viability. 


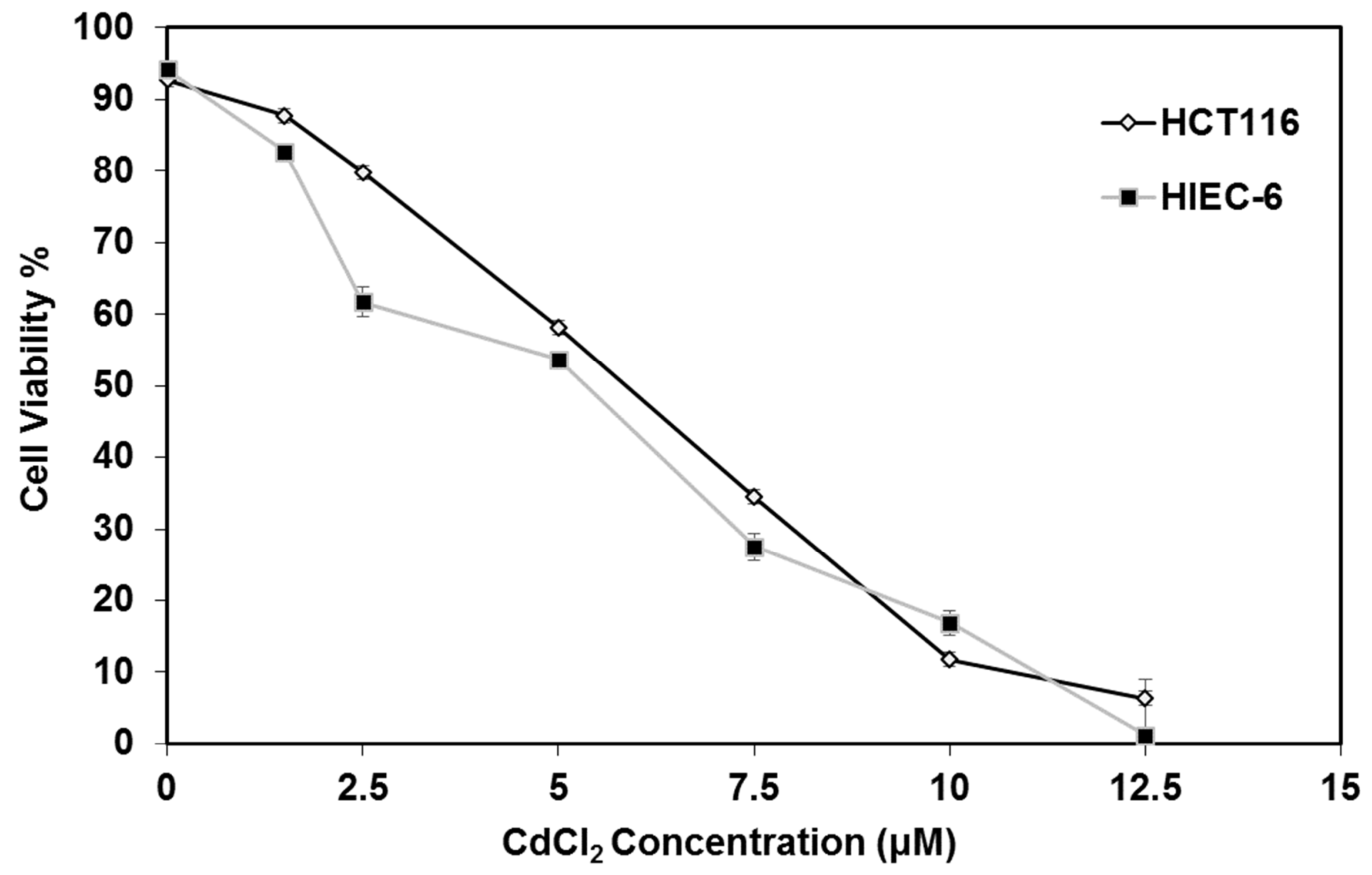


Figure 5.1: Cytotoxic effect of cadmium chloride on HCT116 and HIEC-6 cells. Cells were cultured with increasing concentrations $(0,2.5,5,7.5,10$ and $12.5 \mu \mathrm{M})$ of cadmium chloride for $24 \mathrm{hrs}$. Cell viability was determined based on the MTT assay. DMSO controls were subtracted from the total viability measures in the MTT assay. Each point represents the means \pm S.E.M of three experiments with three replicates per concentrations. 


\subsubsection{Cadmium exposure induced ROS using (DCFDA, also known as $\mathrm{H}_{2}$ DCFDA).}

ROS production was observed using the cell permeant reagent 2',7'dichlorofluorescin diacetate (DCFDA; also known as $\mathrm{H}_{2}$ DCFDA). DCFDA is a fluorogenic dye that measures ROS activity within the cell. $\mathrm{H}_{2}$ DCF-DA enters cells where it is transformed into 2,7-dichlorodihydrofluorescein $\left(\mathrm{H}_{2} \mathrm{DCF}\right)$ by intracellular stearases. $\mathrm{H}_{2} \mathrm{DCF}$ is then oxidized into fluorescent DCF by hydrogen peroxide. Thus, the fluorescent intensity is proportional to the amount of peroxides produced by the cells. Concentrations were selected based on previous data from MTT assay (Fig. 5.1), illustrating the optimal concentrations for HIEC-6 and HCT116 to be exposed. The results showed that when HIEC-6 cells were treated with cadmium, the cells become more fluorescent with concentrations of cadmium chloride ranging from $2.5-10 \mu \mathrm{M}$, indicating an elevated level of ROS (Fig. 5.2). The control in HIEC-6 showed no fluorescent activity compared to the group that are exposed to cadmium. Interestingly, the control showed a small number of fluorescent cells in HCT116 cells, while cadmium-induced cells showed a higher amount of fluorescence as the concentration of cadmium increased from 2.5 to $5 \mu \mathrm{M}$. However, HCT116 cell density decreased at $10 \mu \mathrm{M}$ in both cell lines Fig. 5.2. 


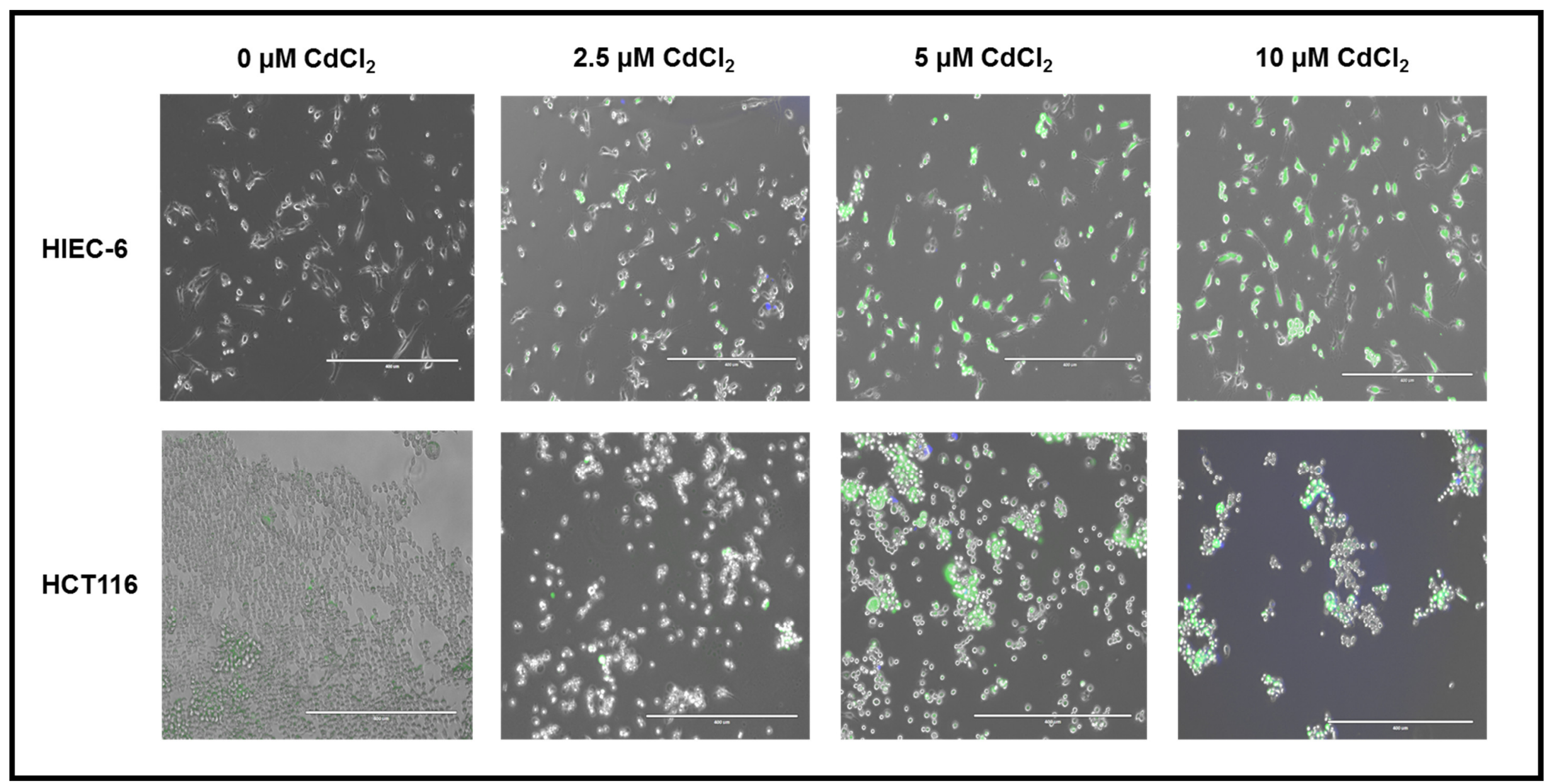


Figure 5.2: Representive images of the effect of cadmium treated cells at different concentrations in HIEC-6 and HCT116. The cells were then stained with dichlorofluorescein-diacetate $\left(\mathrm{DCFH}_{2}-\mathrm{DA}\right)$. The green fluorescent cells represent the intracellular ROSgeneration in HIEC-6 and HCT116 treated at $0,2.5,5$ and $10 \mu \mathrm{M}$ of $\mathrm{CdCl}_{2}$. The images illustrated an increase in cell death at the concentration of cadmium increased. Moreover, more cells were observed to detach and floating in the media with the increase of cadmium concentration ( scale bar $=400 \mu \mathrm{m}$ at $10 \mathrm{x}$ magnification). 


\subsubsection{Expression profile of transcription factors in cadmium-treated cells}

In the present study, we investigated the mRNA expression using qPCR for selective transcription factors that are induced during stress. In all, in the cadmium-exposed cells, our results showed an increase in the gene expression levels at $5 \mu \mathrm{M}$ cadmium concentration, of the following stress-related genes: GRP78, HIF1 $\alpha$ and $M T 1$, and $10 \mu \mathrm{M}$ concentration for NFE2L1 and NFE2L2 in HCT116 and HIEC-6. HIEC-6 cells showed a higher expression when treated with cadmium (Fig. 5.2), as well as an upregulation of GRP78, HIF1 $\alpha, N F E 2 L 1$, and NFE2L2 mRNA expression. HCT116 cells showed a higher tolerance towards cadmium (Fig.5.1) according to the MTT assay. In addition, we investigated how cadmium exposed cells stimulated the expression of a protective molecule such as metallothionein 1 (MT1) (Fig.5.4). Expression of MT1 mRNA in HIEC6 to $5 \mu \mathrm{M}$ of $\mathrm{CdCl}_{2}$ illustrated the highest gene expression compared to $10 \mu \mathrm{M}$ in HCT116. The gene expressions in HIEC-6 cells were higher than that in HCT116 cells (Fig.5.4). 


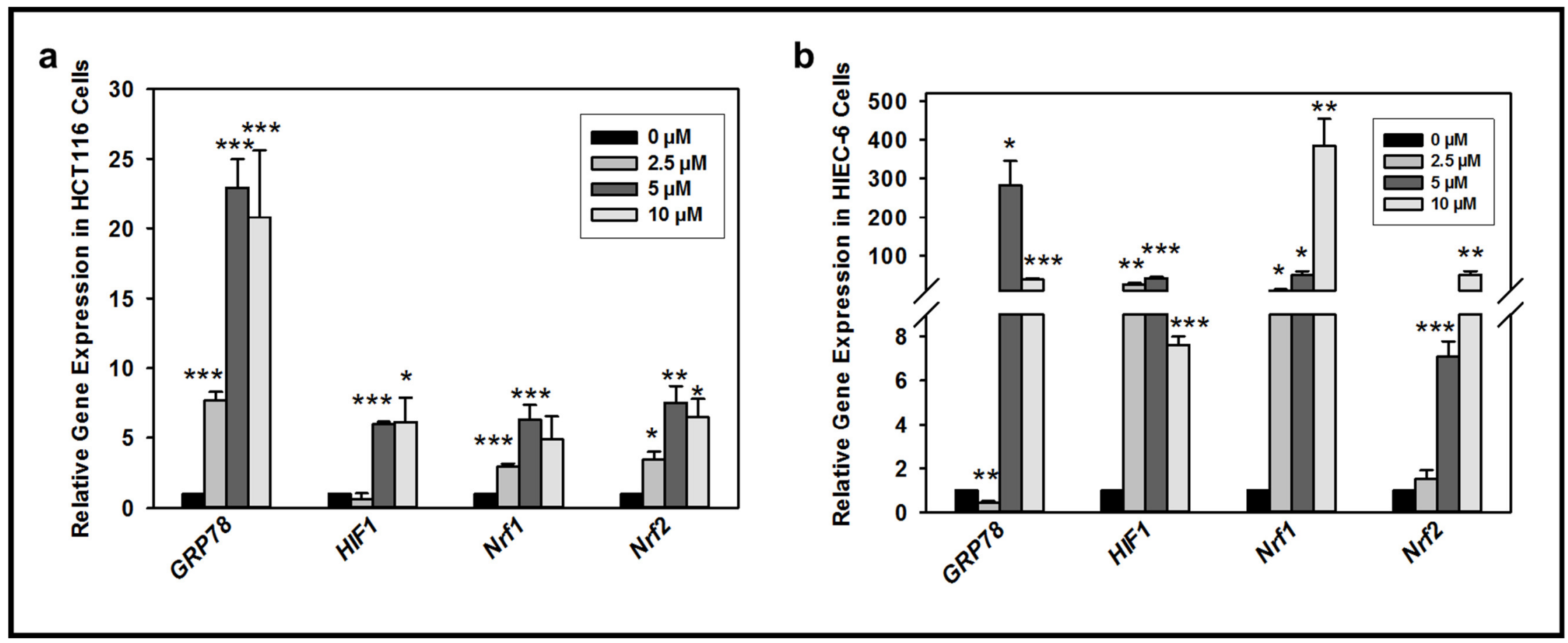

Figure 5.3: The effect of cadmium chloride $\left(\mathrm{CdCl}_{2}\right)$ on GRP78, HIF1 $\alpha$, NFE2L1 and NFE2L2 gene expression in a) human intestinal epithelium cells 6 (HIEC-6) and b) human colon cancer adenocarcinoma (HCT116) cells. HIEC-6 and HCT116 cells were treated with 0, 2.5, 5, and $10 \mu \mathrm{M} \mathrm{CdCl}_{2}(24 \mathrm{hrs})$. Cells without treatment were used as controls. Total RNA was isolated from HIEC-6 and HCT116 cells, converted to cDNA and analyzed by qPCR using gene-specific primers for GRP78, HIF1 $\alpha, N F E 2 L 1$ and NFE2L2 and target 
genes. The level of target genes of each mRNA was normalized to the GAPDH mRNA. Data are the means from three independent experiments \pm S.E.M. Data analysis was done using one-way ANOVA followed by Dunnett's test; ${ }^{*} \mathrm{p}<0.05,{ }^{* *} \mathrm{p}<0.01,{ }^{* * *} \mathrm{p}<0.001$. 


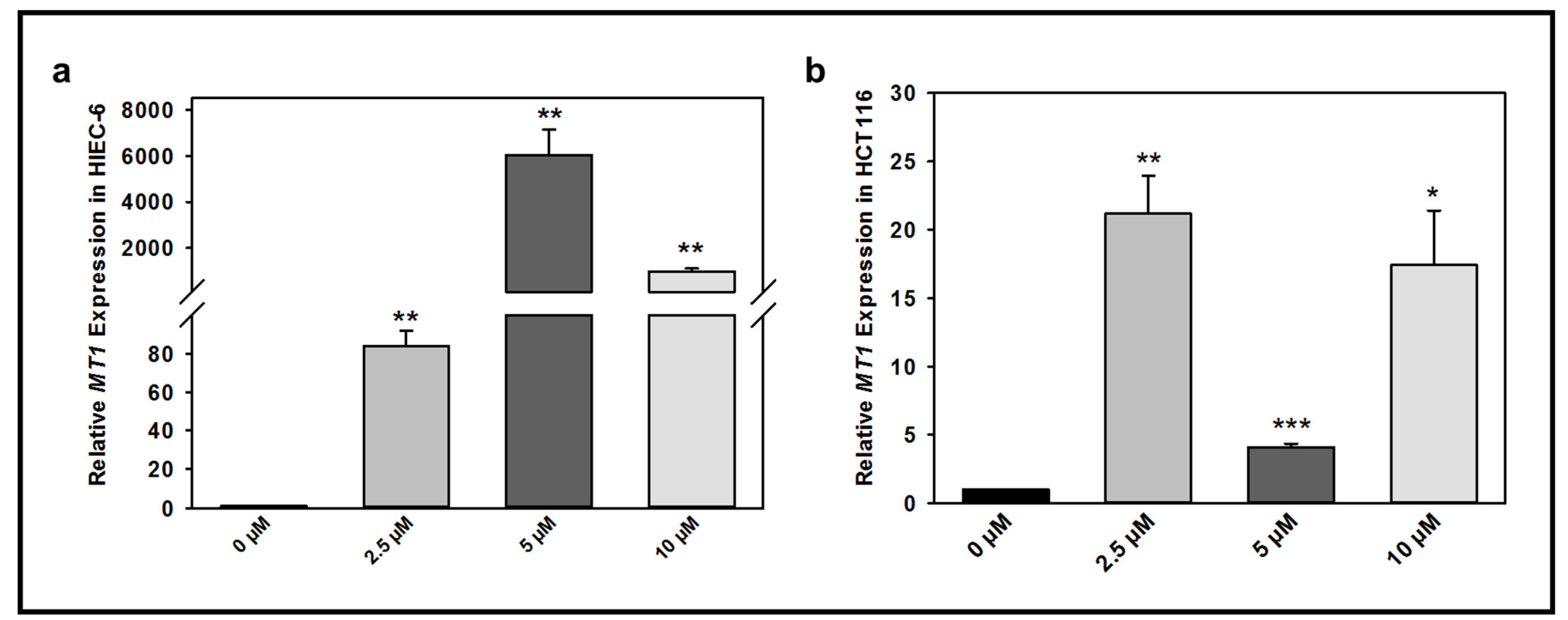


Figure 5.4: The effect of cadmium chloride $\left(\mathrm{CdCl}_{2}\right)$ on $M T 1$ gene expression in a) human intestinal epithelium cell 6 (HIEC-6) and b) human colon cancer adenocarcinoma (HCT116) cells. HIEC-6 and HCT116 cells were treated with 0, 2.5, 5, and $10 \mu \mathrm{M} \mathrm{CdCl} 2(24 \mathrm{hrs})$. Cells without treatment were used as controls. Total RNA was isolated from HIEC-6 and HCT116 cells, converted to cDNA and analyzed by qPCR using gene-specific primers for a MTl target gene. The level of each target gene was normalized to the GAPDH mRNA. Data are the means from three independent experiments \pm S.E.M. Data analysis was done using one-way ANOVA followed by Dunnett's test; ${ }^{*} \mathrm{p}<0.05, * * \mathrm{p}<0.01, * * * \mathrm{p}<0.001$. 


\subsubsection{Induction of WNT gene expression in cadmium-treated cells}

The expression patterns of PORCN, WLS, WNT3A and WNT5A at the level of the gene in HCT and HIEC-6 cells, in response to cadmium treatment, were investigated. In cadmium-exposed cells, WLS mRNA showed a decrease in expression levels as compared to that in controls (Fig.5.5). However, PORCN, WNT3A, and WNT5A demonstrated upregulation at $5 \mu \mathrm{M}$ concentration (Fig. 5.4). The down regulation of PORCN, WNT3A, and WNT5A mRNA followed a dose-dependent trend in cadmium-treated cells; with decreases at $2.5 \mu \mathrm{M}$ in their levels of expression with increased cadmium concentration. The extent of the upregulation was different between both cell lines. HCT116 cells showed a higher vulnerability toward cadmium compared to HIEC-6 which showed a lower vulnerability (Fig. 5.5). HIEC-6 cells showed down regulation of $W L S$ (Fig. 5.4) which explains their moderate resistance towards cadmium-induced cell death. 


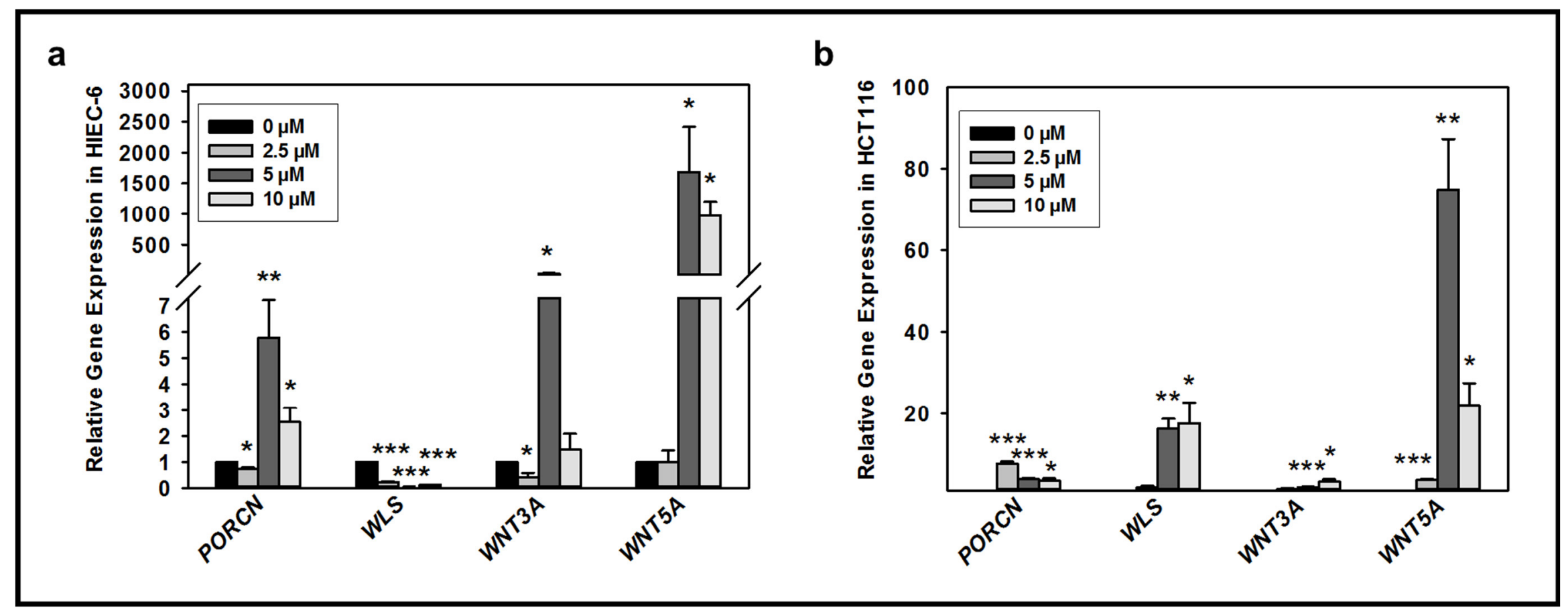


Figure 5.5: The effect of cadmium chloride $\left(\mathrm{CdCl}_{2}\right)$ on PORCN, WLS, WNT3A and WNT5A gene expression in a) human intestinal epithelium cell 6 (HIEC-6) cells and b) human colon cancer adenocarcinoma (HCT116) cells. HIEC-6 and HCT116 cells were treated with $0,2.5,5$, and $10 \mu \mathrm{M} \mathrm{CdCl}_{2}$ (24 hrs). Cells without treatment were used as controls. Total RNA was isolated from HCT116 cells, converted to cDNA and analyzed by qPCR using gene-specific primers for PORCN, $W L S, W N T 3 A$ and $W N T 5 A$ and target genes. The level of target genes of each mRNA was normalized to the GAPDH mRNA. Data are the means from three independent experiments \pm S.E.M. Data analysis was done using one-way ANOVA followed by Dunnett's test; ${ }^{*} \mathrm{p}<0.05,{ }^{* *} \mathrm{p}<0.01, * * * \mathrm{p}<0.001$. 


\subsubsection{Immunoblotting analysis of transcription factors and WNT proteins}

In HIEC-6 cells, GRP78 protein levels increased with increasing cadmium concentration while the protein levels of HIF1 $\alpha$, MT1, NFE2L1 and NFE2L2 showed up regulation with increased cadmium exposure at $5 \mu \mathrm{M}$ and decreased with $10 \mu \mathrm{M}$ (Fig. 5.7). In HIEC-6 cells, both PORCN and WLS showed an upregulation with increased cadmium exposure (Fig. 5.6 (a-h) and (i and j)). However, $\beta$-catenin levels were upregulated with increased cadmium exposure at $5 \mu \mathrm{M}$ and reduction with $10 \mu \mathrm{M}$ (Figs. 5.7 (a-h) and (i and j)). On the contrary, HIEC-6 cells had a higher expression for all transcription factors (GRP78, HIF1 $\alpha$, NFE2L1 and NFE2L2) as well as all the WNT proteins ( $\beta$-catenin, PORCN, WLS and WNT3A) compared to HCT116 cells during cadmium exposure. Interestingly, MT1 expression showed a higher expression at $5 \mu \mathrm{M}$ cadmium; however, the expression of MT1 showed similar patterns between both HIEC-6 and HCT116 cell lines (Fig. $5.9 \mathrm{a}$ and b). 


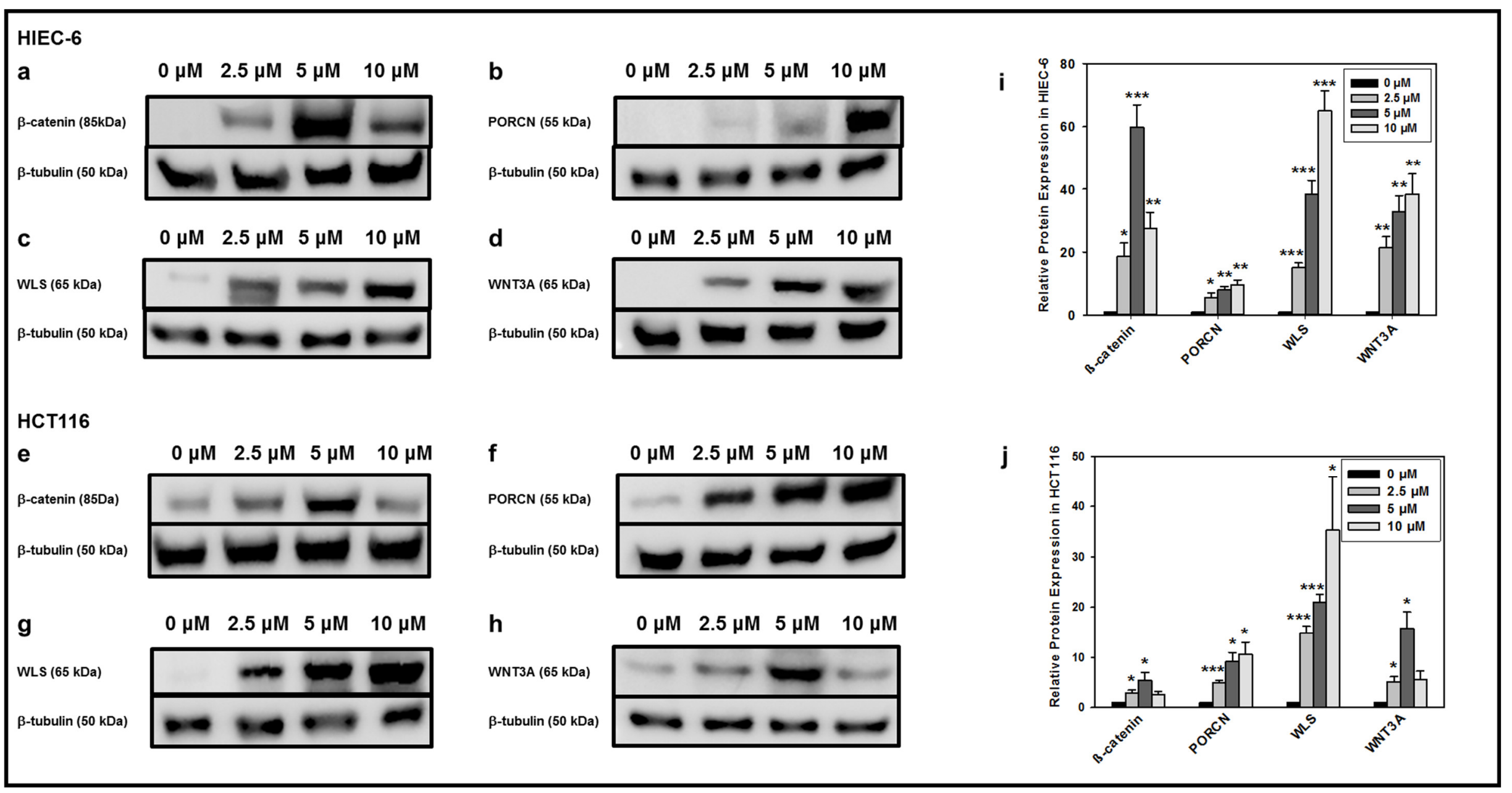

Figure 5.6: The effect of $\mathrm{CdCl}_{2}$ on $\square$-catenin, PORCN, WLS and WNT3A protein expression in (a-d) human epithelium intestinal cells (HIEC-6) and (e-h) human colorectal cancer tumor (HCT116). Cells were treated with $\mathrm{CdCl}_{2}$ at $0,2.5,5$, and $10 \mu \mathrm{M}$ concentrations. a) 
The Western blot analysis showed that (a-d) and (e-h) corresponding to $\beta$-catenin, PORCN, WLS and WNT3A protein expression in HIEC-6 and HCT116 cells respectively. Figure (i) and (j) correspond to the densitometric quantification profile of genes in HIEC-6 and HCT116 cells respectively. The level of protein expression of each sample was normalized to $\beta$-tubulin. Data are the mean band densities from three independent experiment \pm S.E.M. Data analysis was done using one-way ANOVA followed by Dunnett's test; *p $<0.05, * *$ $\mathrm{p}<0.01, * * * \mathrm{p}<0.001$. 


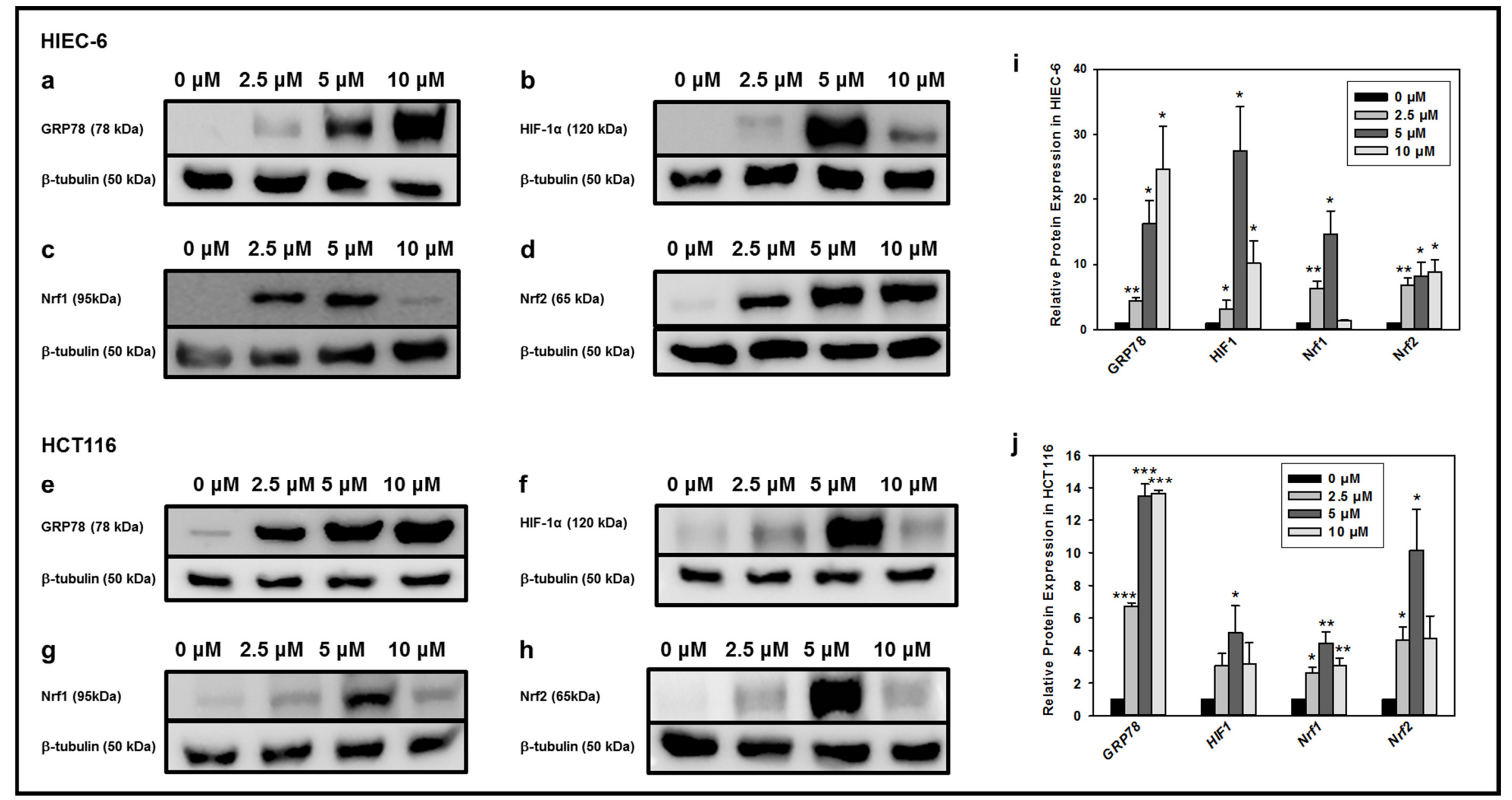


Figure 5.7: The effect of $\mathrm{CdCl}_{2}$ on GRP78, HIF1 $\square$, NFE2L1 and NFE2L2 protein expression in (a-d) human epithelium intestinal cells (HIEC-6) and (e-h) human colorectal cancer tumor (HCT116). Cells were treated with $\mathrm{CdCl}_{2}$ at $0,2.5,5$, and $10 \mu \mathrm{M}$ concentrations. Figure (i) and (j) correspond to the densitometric quantification profile expression of genes in HIEC-6 and HCT116 cells respectively. The level of protein expression of each sample was normalized to $\beta$-tubulin. Data are the mean band densities from three independent experiment \pm S.E.M. Data analysis was done using one-way ANOVA followed by Dunnett's test; ${ }^{*} \mathrm{p}<0.05,{ }^{* *} \mathrm{p}<0.01, * * * \mathrm{p}<0.001$ 


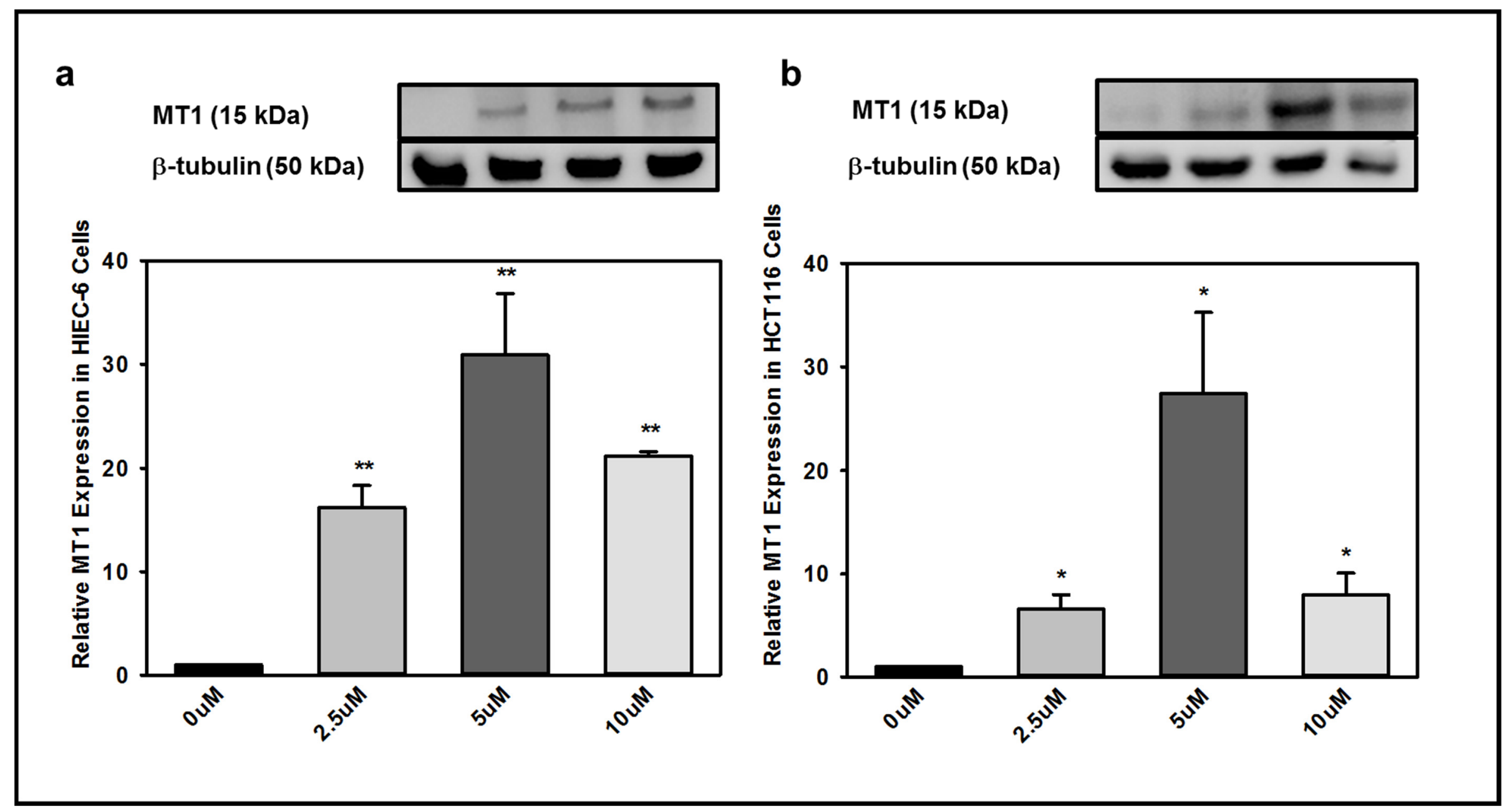


Figure 5.8: The effect of $\mathrm{CdCl}_{2}$ on MT1 gene expression in a) human epithelium intestinal cells (HIEC-6) and b) human colorectal cancer tumor (HCT116). Cells were treated with $\mathrm{CdCl}_{2}$ at $0,2.5,5$, and $10 \mu \mathrm{M}$ concentrations. The corresponding densitometric quantification profile represents the measurement of protein expression. The level of protein expression of each sample was normalized to $\beta$-tubulin. Data are the mean band densities from three independent experiment \pm S.E.M. Data analysis was done using one-way ANOVA followed by Dunnett's test; ${ }^{*} \mathrm{p}<0.05, * * \mathrm{p}<0.01, * * * \mathrm{p}<0.001$. 


\subsection{Discussion}

Exposure to cadmium will result in the inhibition of apoptosis and DNA repair, the stimulation of cell proliferation, and the promotion of cancer in a number of tissues (Templeton and Liu, 2010; Von Zglinicki et al., 1992). At the cellular level, long-term low doses of cadmium exposure induced damage to DNA and cell membranes by inhibiting different types of DNA repair mechanisms, and inducing apoptosis in mammalian cells (Genchi et al., 2020). Cancer cell grow in the absence of signals, they become desentize to signals that constrain their growths, while normal cell grow when they receive signals. In the MTT assay HIEC-6 cells more cell survived compared to HCT116, this can be explained due to the cancer cell have more genetic changes compared to HIEC-6 cell, this results in different responses to cadmium treatments. The accumulation of genetic and epigenetic alterations in cancers cells gives them with undesirable proliferative and metastic potential. However, there modifications can also create a barrier for cancer cells with vulnerabilities such that it's possible to identify a specific genes whose function is more or less critical for the viability of cancer cells than for a normal cells (Li et al., 2007). In this study, the MTT assay was used for cell viability to assess the cytotoxicity of the cadmium chloride $\left(\mathrm{CdCl}_{2}\right)$ on HIEC-6 and HCT116 cells. Our data from this assay demonstrated a clear concentration-response with regard to the cytotoxicity of cadmium chloride in both cell lines. In Figure 5.1, the data indicated there is a gradual decrease in viability of HIEC-6 and HCT116 cells, as the concentration of $\mathrm{CdCl}_{2}$ increased (Fig. 5.1). Furthermore, treatment with cadmium chloride resulted in cell death at concentrations higher than $5 \mu \mathrm{M}$. Cadmium can cause a number of lesions in many organs, such as the kidney and bones, and acute cadmium ingestion can also cause gastrointestinal tract erosion 
(WHO, 1992). However, the main mechanisms of toxicity of cadmium have not been determined. In the present study, we investigated the effect of cadmium induced exposure that ranged from $0,2.5,5,7.5,10$ and $12.5 \mu \mathrm{M}$, cadmium accumulation in both cell lines was found significantly similar.

\subsubsection{Induction of ROS by cadmium chloride}

To test whether oxidative stress plays a key role in cadmium chloride-induced toxicity in HIEC-6 and HCT116 cells, we used the dichlorofluorescene-diacetate $\left(\mathrm{H}_{2}\right.$ DCFDA $)$ assay to measure the generation of ROS upon cell treatment. Many reports showed that heavy metal treatment, including $\mathrm{Cd}$, induce toxicity associated with increased production of ROS leading to severe oxidative stress in cells (Mostafa et al., 2019). In this thesis, our results showed as the concentration of $\mathrm{CdCl}_{2}$ increases the amount of fluorescent cells, indicating the generation of high amounts of ROS in both cell lines (Fig 5.2). It is well known that the generation of ROS, induced by $\mathrm{Cd}$ exposure, can play a role in $\mathrm{Cd}$ induced cell cycle arrest. This information led us to hypothesize that $\mathrm{Cd}$ exposure could play a role in altering WNT signalling activation. $\mathrm{ROS}$ induced by $\mathrm{CdCl}_{2}$ participated in the effects of $\mathrm{CdCl}_{2}$ on the WNT signalling pathway. Further, the morphology of both cell lines were altered showing a loss of adhesions and cells become more round as the concentration of $\mathrm{Cd}$ this could be explained by loss of desmosome that allow the cell to cell contact. Moreover, HCT116 cells showed demolishing of the cell viability, we hypothesize that cadmium could lead to depletion of catalytic enzymes including gluthione and antixodant response genes.

\subsubsection{Cadmium altered WNT gene and protein expression}


There are multiple external triggers that induce oxidative stress that have direct or indirect effects on responses in the GI tract including cadmium; organic solvents; and pesticides are common exogenous sources of ROS (Bhattacharyya, et al., 2014). In HIEC6 and HCT116 cells, both PORCN and WLS gene and protein expression were highly upregulated in the presence of cadmium. Our previous work indicated that ER stress, oxidative stress and hypoxia upregulate both gene and protein expressions of these two proteins (Mohamed et al., 2021). We hypothesized that cadmium exposure, at acute level, could alter both gene and protein expression of PORCN and WLS through the generation of ROS, as our data indicated in Figure 5.2. Furthermore, PORCN and WLS gene and protein expression were upregulated with increasing concentrations of cadmium (Figs. 6 and 8).

In addition, we investigated the effects of cadmium chloride on WNT3A and $\beta$ catenin. Since both WNT3A and WNT5A are WNT family members which play key roles in regulating pleiotropic cellular functions, we decided to investigate their expression upon cadmium exposure. Accumulating evidence suggests that WNT3A promotes or suppresses tumor progression via the canonical WNT signaling pathway through a transcription coactivator called $\beta$-catenin (Milkels and Nusse, 2006). Our data strongly suggest that WNT3A and $\beta$-catenin protein levels are upregulated in both HIEC-6 and HCT116 cells upon cadmium exposure (Figs. 6 and 8). Furthermore, we tested mRNA expression of WNT3A and WNT5A when cells were exposed to cadmium chloride and our results showed upregulation as well in the gene expression (Fig. 5.2). It is known that $\beta$-catenin acts as a molecular switch in response to the cellular redox status. Aberrant WNT/ $\beta$-catenin signaling can contribute to the carcinogenesis of intestinal, lung or kidney epithelia, either 
by mutations of its signaling components and/or disruption of linked signaling networks (Thevenod and Chakraborty, 2010).

\subsubsection{Effect of cadmium on transcription factors}

Cells are able to resist the detrimental effects of redox stress via the activation of the transcription factors involved in the antioxidant response, when exposed to toxins such as Cd (D'Autreaux and Toledano, 2007). To reveal the effects of cadmium exposure on different transcription factors, we examined the mRNA and protein expression levels of GRP78, HIF1 $\alpha$, MT1, NFE2L1, and NFE2L2. We detected significantly increased of GRP78, HIF1 $\alpha$, MT1, NFE2L1, and NFE2L2 in response to toxic levels of cadmium at 5 $\mu \mathrm{M}$ (Fig. 5.2, 5.4-5.9). It's well known that cadmium induces the expression of Grp78, most likely via the phosphorylation of eIF2 (alpha) and the resultant translation of ATF4. This ER stress response plays a role in protection against cadmium cytotoxicity in renal epithelial cells (Liu et al., 2006) and this might also be true in HCT116 and HIEC-6 cells. Our data showed gene and protein expression of HIF $1 \alpha$ is upregulated upon exposure of cells to cadmium. This is not surprising, since HIF1 $\alpha$ play a protective role against cadmium-induced cytotoxicity in mouse embryonic fibroblasts by maintaining metallothionein and antioxidant activity levels (Jing et al., 2012). Similarly, NFE2L2 and NFE2L1 which bind to AREs may be important in the adaptive response to survive the oxidative stress generated by cadmium (Venugopal and Jaiswal, 1998).

The mechanism that underlies the activation of transcription factors by cadmium could be explained by the cadmium activating physiological second messenger systems, in 
particular $\mathrm{Ca}^{2+}$, and reactive oxygen species (ROS) which control key $\mathrm{Ca}^{2+}$ - and redoxsensitive molecular switches dictating cell function and fate (Thévenod and Lee,2013). Previous studies indicated that severe $\mathrm{ROS} / \mathrm{Ca}^{2+}$ signals activate cell death effectors (ceramides, ASK1-JNK/p38, calpains, caspases) and/or cause irreversible damage to vital organelles, such as the mitochondria and ER, whereas low localized $\mathrm{ROS} / \mathrm{Ca}^{2+}$ levels act as second messengers promoting cellular adaptation and survival through signal transduction by ERK1/2, PI3K/Akt-PKB and transcriptional regulators (Ref1-NFE2L2, NF- $\mathrm{BB}, \mathrm{WNT}, \mathrm{AP}-1$, bestrophin-3) (Thévenod and Lee,2013). Other cellular proteins and processes targeted by $\mathrm{ROS} / \mathrm{Ca}^{2+}$ (metallothioneins, Bcl-2 proteins, ubiquitin-proteasome system, ER stress-associated unfolded protein response, autophagy, cell cycle) can evoke either cell death or cell survival depends on the concentration of cadmium exposed cells. It increases the generation of ROS by depleting cellular glutathione (GSH) and antioxidant enzymes, such as superoxide dismutase (SOD) and catalase (CAT) (Bagchi et al., 2002). One hypothesis that addresses how cadmium activates intracellular signaling pathways, such as WNT, proposes that the metal modulates the level of ROS and thus, calcium could be viewed as a second messenger that mediates cadmium-responsive transcription.

\subsection{Conclusions}

At present, there is no unifying concept that might allow the prediction of the effect of cadmium on the expression of a given gene or protein. Whether or not the transcription of an individual gene will be stimulated or impaired by this metal depends on the distinct cellular signalling path affected. Nevertheless, a more integrated view on cellular signaling associated with cadmium toxicity may help to contribute to the development of preventive 
and novel therapeutic strategies for acute and chronic cadmium toxicity. One particular factor or signaling pathway is unable to provide a complete understanding of the impact of cadmium on cellular functions, and one should be aware that conclusions about the significance of that process for cell fate will in effect encompass multiple signaling pathways that will dictate the actual outcome for the cell 


\section{Chapter 6: Discussion and Conclusions}

\subsection{Discussion}

Our current knowledge of how membrane-bound proteins, such as WNT proteins, are processed through the combined actions of chaperones, retention factors, and degradation machinery and vesicle transport proteins is still limited. At present, there are major unsolved questions in how PORCN and WLS aid in the secretion of the WNT proteins. What is the role of the modifications conferred to the WNT proteins by PORCN and WLS that assist in WNT secretion? How are these modifications altered under stress conditions? How do the WNT proteins achieve a folded, export competent state through the aid of post-translational modifications such as glycosylation and lipid modifications? In addition, the signals required for vesicle transport to and from the ER remain obscure for many membrane-bound proteins. More generally, many factors affecting downstream processes in regulated transport to the cell surface have not been elucidated. It is crucial that we gain a more fundamental knowledge of these processes in order to understand all aspects of the WNT secretory pathway.

Accumulating evidence over the past decade highlights the importance of aberrant WNT signaling activation during oncogenic initiation (Nusse, 2012). This pathway is relatively complex, and all its processes and physiological roles are not completely understood. The essential role of this pathway in cell fate and renewal, and most importantly during embryonic development (Nusse, 2008). Consequently, there is a growing interest within the research community in achieving a better understanding of the secretory pathway that modifies the WNT proteins. The upstream events involved in processing the WNT proteins (post-translation modifications such as palmitoyleation and 
glycosylation) (Nusse, 2003), prior to their secretion, are generally less examined. We investigated stresses that are typically found in the tumour microenvironment (hypoxia, oxidative stress, ER stress), since intestinal epithelial stem cells are extremely sensitive to these stresses (Bhattacharyya et al., 2014). As well, cellular stress severely disrupts intestinal epithelial stem cells, leading to abnormal accumulation of unfolded or misfolded proteins in the ER and subsequent development of oxidative, hypoxia, ER stresses (Bhattacharyya et al., 2014; Coleman et al., 2019). This major event, and their corresponding activation of various signaling pathways, includes WNT signaling.

Accumulation of unfolded or misfolded proteins in cells, due to stress, can lead to an intracellular steady-state imbalance (Haynes et al., 2004). Oxidative, hypoxia and ER stresses are increased during the development and progression of tumours and are a doubleedged sword in terms of their regulatory effects on the survival of cells (Blagosklonny, 2004). While stresses and the UPR protect normal cells, they can also induce tumourigenesis and promote the survival and development of tumour cells (Haynes et al., 2004; Blagosklonny, 2004).

Extensive experimental work over the past three decades examined, in depth, the molecular background of the cellular response to stress (Bhattacharyya et al., 2014). However, our understanding on how stress-induced changes to WNT signalling a pathway is still underdeveloped. Insights into the role of stress responses and their effects on WNT signalling pathways will provide the opportunity to develop more efficient medical modalities for pathologies like cancer and chronic illnesses involving the WNT signalling pathways. 
PORCN, WLS and $\beta$-catenin upregulation when treated with hypoxia, oxidative and ER stressors, which correlated more closely with their growth characteristics. HCT116 which is characterized by wildtype p53 and microsatellite instability (Yao et al., 2005). HCT116 cells responded to a higher protein expression compared to HIEC-6 when treated with hypoxia. The driver of the biology of hypoxic tumours is the transcription factor HIF$1 \alpha$ in hypoxic cells which have been shown to express higher levels of enzymes that are responsible for detoxication of cytotoxic drugs and is responsible for altering gene and protein expression, but also may contribute to cancer progression (Carmeliet et al., 1998; Harris, 2002). Furthermore, when HCT 116 treated with ER stressors which resulted in a load of unfolded proteins exceeds the protein folding capacity of the cell (Urra et al., 2016). In response to ER stress, unfolded substrates cause GRP78/BiP to release three ER stress sensor, PERK, ATF6, and IRE1 $\alpha$. Activation of these ER stress sensors blocks the translation of proteins, enhances the expression of protein chaperones, and increases the degradation of ER-resident proteins to resolve the stress (Sano and Reed, 2013).

PORCN, WNT3A, WNT5A, GRP78, HIF1 $\alpha$, MT1, NFE2L1, and NFE2L2 expression is increased by cadmium in HIEC-6 compared to HCT116 cells Fig 5.3. We hypothesize the hyper-activation of the relative gene expression after the exposure of HIEC-6 to cadmium causes acquisition of cancer cell characteristic. Previous studies indicated oxidant adaptive response genes MT1, HO-1 and HIF-1A were also activated in CCT-LC cells culminating in reduced cadmium accumulation, suggesting adaptation to the metal. Overall, these data suggest that exposure of human lung epithelial cells to cadmium causes gaining of cancer cell characteristics (Person et al., 2013). Furthermore, we hypothesize that the hyper-activation of HIEC-6 at gene expression upon exposure to 
cadmium, could be explained due to the hyper-activation of antioxidant responses functions. Furthermore, it has been shown that cadmium transformed or adapted cells are characterized by increased immune to apoptosis (Hart et al., 2001; Achanzar et al., 2002), which may render them more susceptible to accumulation of mutations and neoplastic transformation. This will lead to disruption of apoptosis which is critical in tumor formation and malignant progression, and acquired resistance to apoptosis is a general hallmark of cancer (Aimola et al., 2012).

In this current work, we have analyzed this connection and its relevance to key transcription factors and stressors, measuring the production of $\beta$-catenin as a downstream end result. The overexpression of PORCN, WLS, and WNT3A in HCT116 and HIEC-6 resulted in a higher expression of GRP78, HIF1 $\alpha$, NFE2L1, and NFE2L2. GRP78, HIF1 $\alpha$, MT1, NFE2L1, and NFE2L2 are controlled by a complex array of transcriptional regulators and post-translational modifications that ensure proper transcriptional activity, under basal conditions. Our studies showed that there is a connection between these transcription factors and WNT signalling activation during stress.

Facilitating this process is an ever-expanding knowledgebase of cancer's epigenetic, genetic, and proteomic underpinnings, allowing researchers to develop target-based diagnostic tool as preventative measures could be accomplish from understanding WNT secretory components: PORCN and WNTLESS. The highlights of our findings are that role and function of PORCN and WLS were responding to hypoxia, oxidative stress, ER stress, and environmental toxins (chapter 3 and 5). In addition, hypoxia, oxidative stress, ER stress, and environmental toxins (cadmium) induced PORCN, WNT3A and $\beta$-catenin gene and protein expression in HIEC-6 and HCT116 cells (chapter 3 and 5). Interestedly, 
knockdown of PORCN and WLS by siRNA affected the expression of WNT3A, we concluded that PORCN and WLS are rate limiting step in WNT secretion (Fig. 3.5). 2BHDA and SCD1 inhibitors of palmitoyleation altered the gene and protein expression of PORCN, WLS and WNT3A. These results highlighted that palmitoyleation is essential during WNT secretion (Fig. 3.6 and 4.3). Furthermore, PNGase, an inhibitor of glycosylation, altered the protein expression of WLS. However, WNT3A expression was slightly decreased, we concluded that glycosylation is not a necessarily a rate limiting for WNT secretion (Fig. 3.7). We examined the possible regulation of PORCN, WLS, and WNT3A by GRP78, HIF1 $\alpha$, NFE2L1, and NFE2L2 in response to stressors. Consistent with all the findings, PORCN and WLS thus appears to be novel in WNT signalling pathway that is rate-limiting for WNT secretion and can ultimately be used as distinct factor in early diagnosis of acute cadmium exposure and cancer.

\subsection{Strengths and Limitations}

In the first section of the thesis, we found that both PORCN and WLS gene and protein expression is altered during these stresses. To our knowledge, this is the first time that PORCN and WLS were identified to respond to ER, hypoxia and oxidative stresses. Notably, these findings were observed in HCT116 and HIEC-6 cell lines, implying a clinical relevance in this finding. It's known that WNT3A and its cargo WLS were highly expressed in Paneth cells where it has a crucial role in maintaining LGR5 $5^{+}$colon stem cells at the bottom of the crypt and was observed in patients with colon cancer (Sato et al., 2011). Protein expression of WNT3A, WLS, PORCN and $\beta$-catenin were elevated were detected by western blotting (Fig. 3.4 and 3.5). Furthermore, when we knockdowned of PORCN 
and WLS, the expression of WNT3A was demolished (Fig. 3.5-3.7). PORCN and WLS expression are a rate limiting step for WNT3A expression and can be used as indicator abnormalities in WNT signaling which is associated with colon cancer.

Moreover, a detailed investigation was done in Chapter 4 of this thesis examining the activation of key transcription factors involved in three different stress responses and their role in modulating WNT signalling. In addition, we investigated WNT signalling during exposure to an environmental toxin. Components of the WNT signalling pathway were examined during exposure of a non-carcinogenic (HIEC-6) and a carcinogenic (HCT116) human intestinal cell line to cadmium.

However, the primary limitation of this study is the lack of an in vitro organoid model to to demonstrate the effects of direct exposure to stressors and measure the sensitivity at a tissue level. Nevertheless, as the focus of this study was to unravel the molecular mechanisms by which WNT modulates the signaling response in HCT116 and HIEC-6 cells following stressors, the lack of animal studies (although rendering the work less complete) does not influence the validity of the findings that WNT secretory components in cell-based model systems. This study concludes that PORCN and WLS mediate ER stress signalling their regulation by different transcription factors. Organoids offer an unprecedented opportunity to examine WNT pathway regulation in normal physiology and cancer (Merenda et al., 2020). Organoids established from the human small intestinal epithelium is the one of the best organoid system to study WNT signalling. This model displays remarkable similarity to the structural organization and morphology of their organ of origin. These organoids are characterized by budding structures that harbor Lgr $5+$ stem 
cells at their base, representing intestinal crypts, interposed by domains carrying all differentiated cell types, representing villi (Farin et al., 2016).

For the second section of the thesis, we investigated key "master regulator" transcription factors that are activated during cellular stress. We investigated the role of GRP78, HIF1 $\square$ NFE2L1, and NFE2L2, as potential master regulators of cellular stress in modifying PORCN, WLS, WNT3A and $\beta$-catenin gene and protein expression. These findings uncovered possible mechanisms by which PORCN and WLS may regulate WNT secretion during the stress response. Furthermore, our findings were also observed in both knockdown (loss-of-function) and overexpression (gain-of-function) conditions in cells, strengthening the validity of this study. In addition, using knockdown we confirmed that palmitoyleation of PORCN is very essential for WNT secretion and that palmitoleation of the WNT proteins is stress regulated.

One of the limitations in this study is that we did not examine other posttranslational modifications, such as phosphorylation, of PORCN and WLS. Nevertheless, the staining results by palmitoleation inhibitors (SCD1 and 2BHDA) confirmed that these modifications are required for proper WNT functioning.

In the third part of this thesis, we investigated how cadmium can modify WNT proteins and their downstream target proteins. Our data illustrated that WNT signalling is modified by cadmium treatments in a dose-dependent manner and that concentrations between 5 and $10 \mu \mathrm{M}$ elicits the maximal response in HCT116 and HIEC-6. Signalling cascades are activated during cellular exposure to cadmium. To of the best of our knowledge, PORCN and WLS are both altered when cells are exposed to cadmium, at both 
the protein and gene levels. These results provide the base for future studies of the effects of cadmium exposure on the WNT signalling pathways in HCT116 and HIEC-6 cells.

\subsection{Future Research Directions}

Given the strong evidence showing that PORCN and WLS are modified by cellular stresses, it is important to further characterize the interaction between these two proteins during stress. There is a promising area we needed to investigate one of our findings showed that PORCN knockdown reduced WLS expression (Fig 3.5). Future experiments will focus on WNT signalling when potential interactions between PORCN and WLS are interrupted.

Protein-protein interactions in living cells can be confirmed with yeast two-hybrid systems or BioID analysis. Isothermal titration calorimetry (ITC) can also be conducted to understand the binding affinity, stoichiometry, and thermodynamic parameters between these two proteins. Following the confirmation of direct binding between PORCN and WLS in living cells, identification of the specific binding domains between these two proteins will need to be performed to increase our understanding of their functional relationship to each other and how this interaction facilitates WNT signalling.

The binding domains between these two proteins may be determined by creating serial truncated versions of PORCN and WLS that will be affinity-tagged within mammalian expression vectors. These constructs will be transfected into HIEC-6 cells or L-WNT3A cells and co-immunoprecipitation assays will be performed to identify the mutual binding domains. The binding domain of PORCN to WLS will be used to design multiple, partially overlapping peptides, for peptide array synthesis in order to identify the 
key amino acids that are involved in the interactions; those with the highest PORCNbinding affinity and those with the highest WLS-binding affinity.

Our findings uncovered interesting results, when we knocked down PORCN, WLS expression was demolished, more interesting when we knockdown WLS, PORCN expression was diminished as well (Fig. 3.5). Furthermore, when we inhibited palmitoyleation by 2BHDA, WLS and WNT3A expression was decreased, similarly when we inhibited the N-glycosylation by F-PGNase (Fig. 3.7). Future experiment, we can examine the two N-glycosylation sites of WNT3A at Asn87 and Asn298, by generating a mutation single or double amino acid and replace Gln87 and Gln 298. In addition, we can also investigate if Fz and LPR5/5 expression will be influenced by the mutations.

Enzymology and kinetic studies on PORCN would be a great way to measure PORCN activity, the challenge with working with PORCN, its extremely challenging to obtain a purified recombinant PORCN. Once purified PORCN sample is available, we could measure kinetic parameter, determine physiochemical properties such as $\mathrm{pH}$ and temperature. This information will be helpful for developing a more successful therapeutic targets for diagnosis.

Moreover, we can analyze the PORCN and WLS promoters in reporter assays. Serial truncated versions of these promoters could be constructed and the regulatory enhancer sites that include AREs, HREs or response elements that control ER stress-related protein gene expression. 


\section{References}

Abrami, L., Kunz, B., Iacovache, I., and van der Goot, F. G. (2008). Palmitoylation and ubiquitination regulate exit of the WNT signaling protein LRP6 from the endoplasmic reticulum. ProcNatl Acad Sci, 105(14), 5384-5389. https://doi.org/10.1073/pnas.0710389105.

Achanzar, W. E., Webber, M. M., \& Waalkes, M. P. (2002). Altered apoptotic gene expression and acquired apoptotic resistance in cadmium-transformed human prostate epithelial cells. The Prostate, 52(3), 236-244. https://doi.org/10.1002/pros.10106

Akesson, A., Julin, B., \& Wolk, A. (2008). Long-term dietary cadmium intake and postmenopausal endometrial cancer incidence: a population-based prospective cohort study. Cancer research, 68(15), 6435-6441. https://doi.org/10.1158/0008-5472.CAN-080329

Aimola, P., Carmignani, M., Volpe, A. R., Di Benedetto, A., Claudio, L., Waalkes, M. P., van Bokhoven, A., Tokar, E. J., \& Claudio, P. P. (2012). Cadmium induces p53dependent apoptosis in human prostate epithelial cells. PLoS ONE, 7(3), e33647. https://doi.org/10.1371/journal.pone.0033647

Arey, B.J., and López, F.J. (2011). Are circulating gonadotropin isoforms naturally occurring biased agonists? Basic and therapeutic implications. Rev Endocr Metab Disord, 12, 275-288. https://doi.org/10.1007/s11154-011-9188-y 
Angers, S., and Moon, R.T. (2009). Proximal events in WNT signal transduction. Nat Rev Mol Cell Biol, 10(7), 468-77. doi: 10.1038/nrm2717.

Arnold, R. S., Shi, J., Murad, E., Whalen, A. M., Sun, C. Q., Polavarapu, R., Parthasarathy, S., Petros, J. A., and Lambeth, J. D. (2001). Hydrogen peroxide mediates the cell growth and transformation caused by the mitogenic oxidase Nox1. Proc Natl Acad Sci, 98(10), 5550-5555. https://doi.org/10.1073/pnas.101505898.

Ashcroft, F.M., and Roper, J. (1993). Transporters, channels andhuman disease, Curr Opin Cell Bio, 5, 677-683. https://doi.org/10.1016/0955-0674(93)90139-H.

Axelrod J. D. (2001). Unipolar membrane association of Dishevelled mediates Frizzled planarcell polarity signaling. Genes Dev, 15(10),1182-1187. https://doi.org/10.1101/gad.890501.

Baker, N.E. (1988). Transcription of the segment-polarity gene wingless in the imaginal discs of Drosophila, and the phenotype of a pupal-lethal wg mutation. Dev, 102(3), 48997. PMID: 3181031.

Bagchi, D.; Stohs, S.J.; Downs, B.W.; Bagchi, M.; and Preuss, H.G. (2002). Cytotoxicity and oxidative mechanisms of different forms of chromium. Tox, 180 (1), 522. doi: $10.1016 / \mathrm{s} 0300-483 \times(02) 00378-5$. 
Balaban, R.S., Nemoto, S., and Finkel, T. (2005). Mitochondria, oxidants, and aging. Cell, 120(4),483-95. doi: 10.1016/j.cell.2005.02.001.

Bartscherer, K., Pelte, N., Ingelfinger, D., and Boutros, M. (2006). Secretion of WNT ligands requires Evi, a conserved transmembrane protein. Cell, 125 (3) 523-533. https://doi.org/10.1016/j.cell.2006.04.009.

Bartoszewski, R., Rab, A., Fu, L., Bartoszewska, S., Collawn, J., and Bebok, Z. (2011). CFTR expression regulation by the unfolded protein response. Meth Enzymol, 491, 3-24. https://doi.org/10.1016/B978-0-12-385928-0.00001-8.

Baselt, R.C., \& Cravey, R.H. (1995). Disposition of Toxic Drugs and Chemicals in Man. 4th Edn. Chicago, IL: Year Book Medical Publishers;. pp. 105-107.

Belden, W. J., and Barlowe, C. (2001). Deletion of yeast p24 genes activates the unfolded protein response. Mol Biol Cell, 12(4), 957-969. https://doi.org/10.1091/mbc.12.4.957

Belenkaya, T.Y., Wu, Y., Tang, X., Zhou, B., Cheng, L., Sharma, Y.V., Yan, D., Selva, E.M., and Lin, X. (2008). The retromer complex influences WNT secretion by recycling WNTless from endosomes to the trans-Golgi network. Dev Cell, 14(1),120-131. doi: 10.1016/j.devcel.2007.12.003. 
Bernales, S., Papa, F.R., and Walter, P. (2006). Intracellular signaling by the unfolded protein response. Annu Rev Cell Dev Biol, 22, 487-508. doi: 10.1146/annurev.cellbio.21.122303.120200.

Bhattacharyya, A., Chattopadhyay, R., Mitra, S., and Crowe, S. E. (2014). Oxidative stress: an essential factor in the pathogenesis of gastrointestinal mucosal diseases. Physiol. Rev, 94(2), 329-354. https://doi.org/10.1152/physrev.00040.2012.

Bilić, J., Huang, Y.L., Davidson, G., Zimmermann, T., Cruciat, C.M., Bienz, M., and Niehrs, C. (2007). WNT induces LRP6 signalosomes and promotes Dishevelled-dependent LRP6 phosphorylation. Science, 316, 1619-1622. https://doi.org/10.1126/science.1137065.

Biswas, M., and Chan, J. Y. (2010). Role of Nrf1 in antioxidant response elementmediated gene expression and beyond. Toxicol Appl Pharmacol, 244(1), 16-20. https://doi.org/10.1016/j.taap.2009.07.034.

Blagosklonny, M.V. (2004). Antiangiogenic therapy and tumor progression. Cancer Cell, 5(1), 13-17. doi: 10.1016/s1535-6108(03)00336-2.

Bodine, P.V . (2008). WNT signaling control of bone cell apoptosis. Cell Res, 18(2), 24853. https://doi.org/10.1038/cr.2008.13. 
Bolton, J.L, Trush, M.A., Penning, T.M., Dryhurst, G., and Monks, T.J. (2000). Role of quinones in toxicology. Chem Res Toxicol, (3), 135-60. doi: 10.1021/tx9902082.

Boone, J.D., Arend, R.C., Johnston, B.E., Cooper, S.J., Gilchrist, S.A., Oelschlager, D.K., Grizzle, W.E., McGwin, G. Jr., Gangrade, A., Straughn, J.M.Jr., and Buchsbaum, D.J. (2015). Targeting the WNT/ $\beta$-catenin pathway in primary ovarian cancer with the porcupine inhibitor WNT974. Lab Invest, 96(2), 249-259. doi: 10.1038/labinvest.2015.150.

Boscher, C., Dennis, J.W., and Nabi, I.R. (2011). Glycosylation, galectins and cellular signaling. Curr Opin Cell Biol, 23(4), 383-92. doi: 10.1016/j.ceb.2011.05.001.

Boso, D., Rampazzo, E., Zanon, C., Bresolin, S., Maule, F., Porcù, E., Cani, A., Della Puppa, A., Trentin, L., Basso, G., and Persano, L. (2019). HIF-1 $\alpha /$ Wnt signalingdependent control of gene transcription regulates neuronal differentiation of glioblastoma stem cells. Theranostics, 9(17), 4860-4877. https://doi.org/10.7150/thno.35882.

Cadigan, K.M., and Liu, Y.I. (2006). WNT signaling: complexity at the surface. $J$ Cell Sci, 119(Pt 3), 395-402. doi: 10.1242/jcs.02826.

Calfon, M., Zeng, H., Urano, F., Till, J.H., Hubbard, S.R., Harding, H.P., Clark, S.G., and Ron, D. (2002). IRE1 couples endoplasmic reticulum load to secretory capacity by processing the XBP-1 mRNA. Nature, 415(6867),92-96. doi: 10.1038/415092a. 
Capdevila, J., and Izpisua Belmonte, J.C. (2001). Patterning mechanisms controlling vertebrate limb development. Annu. Rev. Cell Dev. Biol, 17:87-132.

Carini, F., Mazzola, M., Rappa, F., Jurjus, A., Geagea, A.G., Al Kattar, S., Bou-Assi, T., Jurjus, R., Damiani, P., Leone, A., and Tomasello, G. (2017). Colorectal carcinogenesis: role of oxidative stress and antioxidants. Anticancer Res, 37(9), 47594766. doi: 10.21873/anticanres.11882. PMID: 28870894.

Castro, L. F., Wilson, J. M., Gonçalves, O., Galante-Oliveira, S., Rocha, E., and Cunha, I. (2011). The evolutionary history of the stearoyl-CoA desaturase gene family in vertebrates. BMC Evol Biol, 11, 132. https://doi.org/10.1186/1471-2148-11-132.

Chan, J. Y., Kwong, M., Lu, R., Chang, J., Wang, B., Yen, T. S., and Kan, Y. W. (1998). Targeted disruption of the ubiquitous CNC-bZIP transcription factor, Nrf-1, results in anemia and embryonic lethality in mice. The EMBO journal, 17(6), 1779-1787. https://doi.org/10.1093/emboj/17.6.1779.

Chaston, T.B., Lovejoy, D.B., Watts, R.N., and Richardson, D.R. (2003). Examination of the antiproliferative activity of iron chelators: multiple cellular targets and the different mechanism of action of triapine compared with desferrioxamine and the potent pyridoxal isonicotinoyl hydrazone analogue 311. Clin Cancer Res, 9(1),402-14. PMID: 12538494. 
Chepelev, N. L., Bennitz, J. D., Huang, T., McBride, S., and Willmore, W. G. (2011). The Nrf1 CNC-bZIP protein is regulated by the proteasome and activated by hypoxia. PloS ONE, 6(12), e29167. https://doi.org/10.1371/journal.pone.0029167.

Chipurupalli, S., Kannan, E., Tergaonkar, V., D'Andrea, R., and Robinson, N. (2019). Hypoxia induced ER stress response as an adaptive mechanism in cancer. Int. J. Mol. Sci, 20(3), 749. https://doi.org/10.3390/ijms20030749.

Clemens S. (2006). Toxic metal accumulation, responses to exposure and mechanisms of tolerance in plants. Biochimie, 88(11), 1707-1719. https://doi.org/10.1016/j.biochi.2006.07.003

Clevers, H. (2006). WNT/ $\beta$-catenin signaling in development and disease. Cell, 127(3), 469-480. https://doi.org/10.1016/j.cell.2006.10.018

Coleman, O. I., and Haller, D. (2019). ER stress and the UPR in shaping intestinal tissue homeostasis and immunity. Front. immunol, 10,2825. https://doi.org/10.3389/fimmu.2019.02825.

Coombs, G. S., Yu, J., Canning, C. A., Veltri, C. A., Covey, T. M., Cheong, J. K., Utomo, V., Banerjee, N., Zhang, Z. H., Jadulco, R. C., Concepcion, G. P., Bugni, T. S., Harper, M. K., Mihalek, I., Jones, C. M., Ireland, C. M., and Virshup, D. M. (2010). WLS-dependent secretion of WNT3A requires Ser209 acylation and vacuolar acidification. J. Cell. Sci, 123(Pt19), 3357-3367. https://doi.org/10.1242/jcs.072132. 
Connett, R.J., Honig, C.R., Gayeski, T.E., and Brooks, G.A. (1985) Defining hypoxia: a system view of $\mathrm{VO}_{2}$, glycolysis, energetics, and intracellular $\mathrm{PO}_{2} . J$ Appl Physiol, 68(3), 833-42. doi: 10.1152/jappl.1990.68.3.833.

Costa, R., Peruzzo, R., Bachmann, M., Montà, G.D., Vicario, M., Santinon, G., Mattarei, A., Moro, E., Quintana-Cabrera, R., Scorrano, L., Zeviani, M., Vallese, F., Zoratti, M., Paradisi, C., Argenton, F., Brini, M., Calì, T., Dupont, S., Szabò, I., and Leanza, L. (2019). Impaired mitochondrial ATP production downregulates WNT signaling via ER stress induction. Cell Rep, 28(8),1949-1960.e6. doi: 10.1016/j.celrep.2019.07.050.

Coudreuse, D., and Korswagen, H.C. (2007). The making of WNT: new insights into WNT maturation, sorting and secretion. Dev, 134(1),3-12. doi: 10.1242/dev.02699.

Covey, T.M., Kaur, S., Tan Ong, T., Proffitt, K. D., Wu, Y., Tan, P., and Virshup, D. M. (2012). PORCN moonlights in a WNT-independent pathway that regulates cancer cell proliferation. PloS ONE, 7(4), e34532. https://doi.org/10.1371/journal.pone.0034532.

D'Autreaux, B., and Toledano, M.B. 2007. ROS as signalling molecules: mechanisms that generate specificity in ROS homeostasis. Nat Rev Mol Cell Biol, 8(10), 813-824. doi: $10.1038 / \mathrm{nrm} 2256$. 
Danilkovitch-Miagkova, A., Miagkov, A., Skeel, A., Nakaigawa, N., Zbar, B., \& Leonard, E. J. (2001). Oncogenic mutants of RON and MET receptor tyrosine kinases cause activation of the beta-catenin pathway. Mol Cell Biol, 21(17), 5857-5868. https://doi.org/10.1128/MCB.21.17.5857-5868.2001

Darom, A., Bening-Abu-Shach, U., and Broday, L. (2010). RNF-121 is an endoplasmic reticulum-membrane E3 ubiquitin ligase involved in the regulation of beta-integrin. Mol Cell, 21(11), 1788-1798. https://doi.org/10.1091/mbc.e09-09-0774.

Daub, M., Jöckel, J., Quack, T., Weber, C. K., Schmitz, F., Rapp, U. R., Wittinghofer, A., and Block, C. (1998). The RafC1 cysteine-rich domain contains multiple distinct regulatory epitopes which control Ras-dependent Raf activation. Mol Cell Biol, 18(11), 6698-6710. https://doi.org/10.1128/MCB.18.11.6698.

Denko, N.C., Fontana, L.A., Hudson, K.M., Sutphin, P.D., Raychaudhuri, S., Altman, R., and Giaccia, A.J. (2003). Investigating hypoxic tumor physiology through gene expression patterns. Oncogene, 22(37), 5907-5914. doi: 10.1038/sj.onc.1206703.

Dennis, J. W., Nabi, I. R., and Demetriou, M. (2009). Metabolism, cell surface organization, and disease. Cell, 139(7), 1229-1241. https:/doi.org/10.1016/j.cell.2009.12.008.

de Sousa E Melo, F., and Vermeulen, L. (2016). WNT signaling in cancer stem cell biology. Cancers, 8(7), 60. https://doi.org/10.3390/cancers8070060. 
Dhakshinamoorthy, S., Jain, A.K., Bloom, D.A., and Jaiswal, A.K. (2005). Bach1 competes with Nrf2 leading to negative regulation of the antioxidant response element (ARE)-mediated NAD(P)H:quinone oxidoreductase 1 gene expression and induction in response to antioxidants. $J$ Biol Chem, 280(17),16891-900. doi: 10.1074/jbc.M500166200.

Dhanvantari, S., and Loh, Y.P. (2000). Lipid raft association of carboxypeptidase E is necessary for its function as a regulated secretory pathway sorting receptor. $J$ Biol Chem, 275(38), 29887-93. doi: 10.1074/jbc.M005364200.

Dings, J., Meixensberger, J., Jäger, A., and Roosen, K. (1998). Clinical experience with 118 brain tissue oxygen partial pressure catheter probes. Neurosurgery, 43(5), 1082-95. doi: 10.1097/00006123-199811000-00045. PMID: 9802852.

Dorland, W.A.N. 1988. Dorland's illustrated medical dictionary (27 $7^{\text {th }}$ ed.) Philadelphia, Saunders publisher.

Drost, J., van Jaarsveld, R.H., Ponsioen, B., Zimberlin, C., Korving, J., Watering, M, van Boxtel, R., Buijs, A., Sachs, N., Overmeer, R.M., Offerhaus, G.J., Begthel, H., Schwank, G., Logtenberg, G., Cuppen, E., Snippert, H.J., Medema, J.P., Kops., and Clevers, H. (2015). Sequential cancer mutations in cultured human intestinal stem cells. Nature, 521, 43-47. https://doi.org/10.1038/nature14415. 
DuRose, J. B., Scheuner, D., Kaufman, R. J., Rothblum, L. I., and Niwa, M. (2009). Phosphorylation of eukaryotic translation initiation factor 2 alpha coordinates rRNA transcription andtranslation inhibition during endoplasmic reticulum stress. Mol Cell Biol, 29(15), 4295-4307. https://doi.org/10.1128/MCB.00260-09.

Erbel, P. J., Card, P. B., Karakuzu, O., Bruick, R. K., and Gardner, K. H. (2003). Structural basis for PAS domain heterodimerization in the basic helix--loop--helix-PAS transcription factor hypoxia-inducible factor. Proc Natl Acad Sci, 100(26), 15504-15509. https://doi.org/10.1073/pnas.2533374100.

Essers, M.A., de Vries-Smits, L.M., Barker, N., Polderman, P.E., Burgering, B.M., and Korswagen, H.C. (2005). Functional interaction between beta-catenin and FOXO in oxidative stress signaling. Science, 308(5725),1181-4. doi: 10.1126/science.1109083.

Farin, H. F., Jordens, I., Mosa, M. H., Basak, O., Korving, J., Tauriello, D. V., de Punder, K., Angers, S., Peters, P. J., Maurice, M. M., \& Clevers, H. (2016). Visualization of a short-range Wnt gradient in the intestinal stem-cell niche. Nature, 530(7590), 340-343. https://doi.org/10.1038/nature16937

Fernandez, E.L., Gustafson, A.L., Andersson, M., Hellman, B., and Dencker. L. (2003). Cadmium-induced changes in apoptotic gene expression levels and DNA damage in mouse embryos are blocked by zinc. Toxicol Sci. 76,162-170. 
Flanagan, D.J., Vincan, E., and Phesse, T.J. (2019). WNT signaling in cancer: not a binary ON:OFF switch. Cancer Res, 79(23), 5901-5906. doi: 10.1158/0008-5472.CAN$19-1362$.

Filomeni, G., Bolaños, J. P., and Mastroberardino, P. G. (2012). Redox status and bioenergetics liaison in cancer and neurodegeneration. J. Cell Biol, 659645. https://doi.org/10.1155/2012/659645.

Fontana, L., Gentilin, B., Fedele, L., Gervasini, C., and Miozzo, M. (2017). Genetics of Mayer-Rokitansky-Küster-Hauser (MRKH) syndrome. Clin Genet, 91(2), 233-246. doi: $10.1111 /$ cge. 12883 .

Franz, E., Römkens, P., van Raamsdonk, L., \& van der Fels-Klerx, I. (2008). A chain modeling approach to estimate the impact of soil cadmium pollution on human dietary exposure. J Food Protect, 71(12), 2504-2513. https://doi.org/10.4315/0362-028x71.12.2504.

Freedman, S. J., Sun, Z. Y., Poy, F., Kung, A. L., Livingston, D. M., Wagner, G. and Eck, M. J. (2002) Structural basis for recruitment of CBP/p300 by hypoxia-inducible factor-1 alpha. Proc. Natl. Acad. Sci. USA, 99, 5367-5372. 
Friberg, L., Elinder, C., Kjellstrom, T., and Nordberg, G.F. (1985). Cadmium and health: a toxicological and epidemiological appraisal volume 1: exposure, dose, and metabolism. Effects and response. Boca Raton, FL: CRC Press, 23-64.

Fu, J., Jiang, M., Mirando, A. J., Yu, H. M., and Hsu, W. (2009). Reciprocal regulation of WNT and Gpr177/mouse WNTless is required for embryonic axis formation. Proc Natl Acad Sci, 106(44), 18598-18603. https://doi.org/10.1073/pnas.0904894106.

Fulda, S., Gorman, A. M., Hori, O., \& Samali, A. (2010). Cellular stress responses: cell survival and cell death. Int J Cell Biol, 2010, 214074. https://doi.org/10.1155/2010/214074

Galli, L.M., Barnes, T.L., Secrest, S.S., Kadowaki, T., and Burrus, L.W. (2007). Porcupine-mediated lipid-modification regulates the activity and distribution of WNT proteins in the chick neural tube. Dev, 134(18), 3339-48. doi: 10.1242/dev.02881.

Galli, L. M., and Burrus, L. W. (2011). Differential palmit(e)oylation of Wnt1 on C93 and S224 residues has overlapping and distinct consequences. PloS ONE, 6(10), e26636. https://doi.org/10.1371/journal.pone.0026636 
Ganem, N., Godinho, S., and Pellman D. (2009). A mechanism linking extra centrosomes to chromosomal instability. Nature, 460, 278-282.

Gao, B. (2012). WNT regulation of planar cell polarity (PCP). Curr Top Dev Biol, 101, 263-95. doi: 10.1016/B978-0-12-394592-1.00008-9.

Gao, H., Chen, D., Liu, X., Wu, M., Mi, J., and Wang, W. (2013). Polymorphisms and expression of the WNT8A gene in Hirschsprung's disease. Int J Mol Med, 32(3), 647-52. doi: 10.3892/ijmm.2013.1433.

Gao, X., and Hannoush, R.N. (2014). Single-cell imaging of WNT palmitoylation by the acyltransferase porcupine. Nat. Chem. Biol, 10, 61-68. https://doi.org/10.1038/nchembio.1392.

Gatica-Andrades, M., Vagenas, D., Kling, J., Nguyen, T.K, Benham, H., and Thomas, R. (2017). WNT ligands contribute to the immune response during septic shock andamplify endotoxemia-driven inflammation in mice. Blood $A d v, 1,1274-86$. doi: 10.1182/bloodadvances.2017006163. 
Genchi, G., Sinicropi, M. S., Lauria, G., Carocci, A., and Catalano, A. (2020). The effects of cadmium toxicity. Int. J. Environ. Res. Public Health, (11), 3782. https://doi.org/10.3390/ijerph17113782

Globocan. (2018). International Agency for Research on Cancer, World Health Organization.

Groenman, F. A., Rutter, M., Wang, J., Caniggia, I., Tibboel, D., \& Post, M. (2007). Effect of chemical stabilizers of hypoxia-inducible factors on early lung development. $\mathrm{Am}$ J Phys: Lung Cell Mol Physiol, 293(3), L557-L567. https://doi.org/10.1152/ajplung.00486.2006

Grzeschik, K.H., Bornholdt, D., Oeffner, F., König, M., Boente, M.D., Enders, H., Fritz, B., Hertl, M., Grasshoff, U., Höfling, K., Oji, V., Paradisi, M., Schuchardt, C., Szalai, Z., Tadini, G., Traupe, H., and Happle, R. (2007). Deficiency of PORCN, a regulator of WNT signaling, is associated with focal dermal hypoplasia. Nat. Genet, 39,833-835. https://doi.org/10.1038/ng2052. 
Guan, X., and Fierke, C. A. (2011). Understanding protein palmitoylation: biological significance and enzymology. Sci. China Chem , 54(12), 1888-1897. https://doi.org/10.1007/s11426-011$4428-2$.

Hamada, F., Murata, Y., Nishida, A., Fujita, F., Tomoyasu, Y., Nakamura, M., Toyoshima, K., Tabata, T., Ueno, N., and Akiyama, T. (1999). Identification and characterization of E-APC, a novel Drosophila homologue of the tumour suppressor APC. Genes Cells, 4(8), 465-74. doi: 10.1046/j.1365-2443.1999.00272.x.

Hamazaki, J., and Murata, S. (2020). ER-resident transcription factor Nrf1 regulates proteasome expression and beyond. Int JMol. Sci, 21(10), 3683. https://doi.org/10.3390/jims21103683.

Han, Y.L., Sheng, Z., Liu, G.D., Long, L.L., Wang, Y.F., Yang, W.X., and Zhu, J.Q. (2015). Cloning, characterization and cadmium inducibility of metallothionein in the testes of the mudskipper Boleophthalmus pectinirostris. Ecotoxicol. Environ. Saf, 119, 18. doi: 10.1016/j.ecoenv.2015.04.055.

Harding, H.P., Novoa, I., Zhang, Y., Zeng, H., Wek, R., Schapira, M., and Ron, D. (2000). Regulated translation initiation controls stress-induced gene expression in mammalian cells. Mol. Cell, 6(5), 1099-1108. doi: 10.1016/s1097-2765(00)00108-8. 
Harris, A. L. (2002). Hypoxia--a key regulatory factor in tumour growth. Nature reviews. Cancer, 2(1), 38-47. https://doi.org/10.1038/nrc704

Harris, C.M., Sanders, S.A., and Massey, V. (1999). Role of the flavin midpoint potential and NAD binding in determining NAD versus oxygen reactivity of xanthine oxidoreductase. J Biol Chem, 274(8), 4561-9. doi: 10.1074/jbc.274.8.4561. PMID: 9988690.

Hart, B. A., Potts, R. J., \& Watkin, R. D. (2001). Cadmium adaptation in the lung - a double-edged sword? Toxicology, 160(1-3), 65-70. https://doi.org/10.1016/s0300$483 \times(00) 00436-4$

Harterink, M., and Korswagen, H.C. (2012) Dissecting theWNT secretion pathway: key questions on the modification and intracellular trafficking of WNT proteins. Acta Physiol, 204, 8-16.

Hartl, F.U., and Hayer-Hartl, M. (2009). Converging concepts of protein folding in vitro and in vivo. Nat Struct Mol Biol, 16(6), 574-81. doi: 10.1038/nsmb.1591. 
Hartmann, E., Rapoport, T.A., and Lodish, H.F. (1989). Predicting the orientation of eukaryotic membrane-spanning proteins. Proc. Natl. Acad. Sci. U.S.A, 86, 5786-5790. https://doi.org/10.1073/pnas.86.15.5786

Hartwig, A. (2010). Mechanisms in cadmium-induced carcinogenicity: recent insights. Biometals: an international journal on the role of metal ions in biology, biochemistry, and medicine, 23(5), 951-960. https://doi.org/10.1007/s10534-010-9330-4

Haynes, C.M., Titus, E.A., and Cooper, A.A. (2004). Degradation of misfolded proteins prevents ER-derived oxidative stress and cell death. Mol Cell, 15(5), 767-776. doi: 10.1016/j.molcel.2004.08.025.

He, T.C., Sparks, A.B., Rago, C., Hermeking, H., Zawel, L., da Costa, L.T., Morin, P.J., Vogelstein, B., and Kinzler, K.W. (1998). Identification of c-MYC as a target of the APC pathway. Science, 281(5382),1509-1512.

Herz, J., and Strickland, D. K. (2001). LRP: a multifunctional scavenger and signaling receptor. J Clin Invest, 108(6), 779-784. https://doi.org/10.1172/JCI13992. 
Heichsman, F., Smith, L., and Cumberledge, S. (1996). Glycosaminoglycans can modulate extracellular localization of the wingless protein andpromote signal transduction. J. Cell Biol, 135(3), 819-827. https://doi.org/10.1083/jcb.135.3.819.

Hishikawa, Y., Kohno, H., Ueda, S., Kimoto, T., Dhar, D.K., Kubota, H., Tachibana, M., Koji, T., and Nagasue, N. (2001). Expression of metallothionein in colorectal cancers andsynchronous liver metastases. Oncology, 61(2), 162-7. doi: 10.1159/000055368. PMID: 11528256.

Ho, S.Y., and Keller, T.H. (2015). The use of porcupine inhibitors to target WNT-driven cancers. Bioorg Med Chem Lett, 25(23), 5472-6. doi: 10.1016/j.bmcl.2015.10.032.

Hofmann, K. (2000). A superfamily of membrane-bound O-acyltransferases with implications for WNT signaling. Trends Biochem Sci, 25(3),111-112. doi: 10.1016/s09680004(99)01539-X.

Hong, C.F., Chen, W.Y., and Wu, C.W. (2017). Upregulation of WNT signaling under hypoxia promotes lung cancer progression. Oncol Rep, 38(3), 1706-1714. doi: 10.3892/or.2017.5807. 
Hosseini, V., Dani, C., Geranmayeh, M.H., Mohammadzadeh, F., Nazari, Soltan Ahmad, S., and Darabi, M. (2019). WNT lipidation: Roles in trafficking, modulation, and function. J Cell Physiol, 234(6), 8040-8054. doi: 10.1002/jcp.27570.

Houschyar, K. S., Tapking, C., Borrelli, M. R., Popp, D., Duscher, D., Maan, Z. N., Chelliah, M. P., Li, J., Harati, K., Wallner, C., Rein, S., Pförringer, D., Reumuth, G., Grieb, G., Mouraret, S., Dadras, M., Wagner, J. M., Cha, J. Y., Siemers, F., Lehnhardt, M., and Behr, B. (2019). WNT pathway in bone repair and regeneration What doweknow so far. Front. Cell. Dev Bio, 6, 170. https:/doi.org/10.3389/fcell.2018.00170.

Hsu, S.Y., Kudo, M., Chen, T., Nakabayashi, K., Bhalla, A., van der Spek, P.J., van Duin, M., and Hsueh, A.J. (2000). The three subfamilies of leucine-rich repeat-containing G protein-coupled receptors (LGR): identification of LGR6 and LGR7 and the signaling mechanism for LGR7. Mol Endocrinol, 14(8),1257-71. doi: 10.1210/mend.14.8.0510.

Huang, H. C., and Klein, P. S. (2004). The Frizzled family: receptors for multiple signal transduction pathways. Genome Biol, 5(7), 234. https://doi.org/10.1186/gb-2004-5-7-234. 
Hubbi, M.E., and Semenza, G.L. (2015). Regulation of cell proliferation by hypoxiainducible factors. Am J Physiol Cell Physiol, 309(12), C775-C782. https://doi.org/10.1152/ajpcell.00279.2015.

Hybertson, B.M., Gao, B., Bose, S.K., McCord, J.M. (2011). Oxidative stress in health and disease: The therapeutic potential of Nrf2 activation. Mol Aspects Med, 32(4-6), 23446. doi: 10.1016/j.mam.2011.10.006.

Ikonen, E. (2001). Roles of lipid rafts in membrane transport, Curr. Opin. Cell Biol, 13, 470-477. https://doi.org/10.1016/S0955-0674(00)00238-6.

Innonvation Training Network. 2020. Endoplasmic reticulum stress. http:/www.trainers.eu/background/

Iommarini, L., Porcelli, A. M., Gasparre, G., and Kurelac, I. (2017). Non-canonical mechanisms regulating hypoxia-inducible factor 1 alpha in cancer. Front. Oncol, 7, 286. https://doi.org/10.3389/fonc.2017.00286. 
Jan, C. H., Williams, C. C., and Weissman, J. S. (2014). Principles of ER cotranslational translocation revealed by proximity-specific ribosome profiling. Science, 346(6210), 1257521. https://doi.org/10.1126/science.1257521.

Jiang, S., Zhang, E., Zhang, R., and Li, X. (2016). Altered activity patterns of transcription factors induced by endoplasmic reticulum stress. BMC Biochem 17, 8 . https://doi.org/10.1186/s12858-016-0060-2.

Jing, Y., Liu, L. Z., Jiang, Y., Zhu, Y., Guo, N. L., Barnett, J., Rojanasakul, Y., Agani, F., and Jiang, B. H. (2012). Cadmium increases HIF-1 and VEGF expression through ROS, ERK, and AKT signaling pathways and induces malignant transformation of human bronchial epithelial cells. Toxi. Sci, 125(1), 10-19. https://doi.org/10.1093/toxsci/kfr256.

Jones, K.A., and Kemp, C.R. (2008). WNT-induced proteolytic targeting. Genes Dev, 22, 3077-3081. https://doi.org/10.1101/gad.1741008.

Jones, R. B., Dorsett, K. A., Hjelmeland, A. B., and Bellis, S. L. (2018). The ST6Gal-I sialyltransferase protects tumor cells against hypoxia by enhancing HIF-1 $\alpha$ signaling. $J$. Biol. Chem, 293(15), 5659-5667. https://doi.org/10.1074/jbc.RA117.001194. 
Kagi, J.H.R. (1991). Overview of metallothionein. Methods Enzymol, 205, 613-26. https://doi.org/10.1016/0076-6879(91)05145-1.

Kakugawa, S., Langton, P. F., Zebisch, M., Howell, S., Chang, T. H., Liu, Y., Feizi, T., Bineva, G., O'Reilly, N., Snijders, A. P., Jones, E. Y., and Vincent, J. P. (2015). Notum deacylates WNT proteins to suppress signalling activity. Nature, 519(7542), 187192. https://doi.org/10.1038/nature14259.

Kaldis, P., and Pagano, M. (2009). WNT signaling in mitosis. Dev Cell, 17(6),749-50. doi: 10.1016/j.devcel.2009.12.001.

Katoh, M., Hirai, M., Sugimura, T., and Terada, M. (1996). Cloning, expression and chromosomal localization of WNT-13, a novel member of the WNT gene family. Oncogene, 13(4), 873-6. PMID: 8761309.

Katoh, M. (2006). WNT2B: comparative integromics and clinical applications (Review). Int J Mol Med, 16(6), 1103-1108. PMID: 16273293. 
Katoh M. (2017). Canonical and non-canonical WNT signaling in cancer stem cells and their niches: Cellular heterogeneity, omics reprogramming, targeted therapy and tumor plasticity(Review). Int. JClin. Oncol, 51(5), 1357-1369. https://doi.org/10.3892/ijo.2017.4129.

Kestler, H. A., and Kühl, M. (2008). From individual WNT pathways towards a WNT signalling network. Phil Trans Royal Soc London. Ser B, Biol Sci, 363(1495), 1333-1347. https://doi.org/10.1098/rstb.2007.2251

Keupp, K., Beleggia, F., Kayserili, H., Barnes, A. M., Steiner, M., Semler, O., Fischer, B., Yigit, G., Janda, C. Y., Becker, J., Breer, S., Altunoglu, U., Grünhagen, J., Krawitz, P., Hecht, J., Schinke, T., Makareeva, E., Lausch, E., Cankaya, T., Caparrós-Martín, J. A., , Lapunzina, P., Temtamy, S., Aglan, M., Zabel, B., Eysel, P., Koerber, F., Leikin, S., Garcia, K.C., Netzer, C., Schönau, E., Ruiz-Perez, V.L., Mundlos, S., Amling, M., Kornak, U., Marini, J., and Wollnik, B. (2013). Mutations in WNT1 cause different forms of bone fragility. Am. J. Hum. Genet, 92(4), 565-574. https://doi.org/10.1016/j.ajhg.2013.02.010

Kim, Y.J., Kim, E.-H. and Hahm, K.B. (2012), Oxidative stress in inflammation-based gastrointestinal tract diseases: Challenges and opportunities. J. Gastroenterol. Hepatol, 27, 1004-1010. https://doi.org/10.1111/j.1440-1746.2012.07108.x 
Kim, S., Joe, Y., Kim, H. J., Kim, Y. S., Jeong, S. O., Pae, H. O., Ryter, S. W., Surh, Y. J., and Chung, H. T. (2015). Endoplasmic reticulum stress-induced IRE1 $\alpha$ activation mediates cross-talk of GSK-3 $\beta$ and XBP-1 to regulate inflammatory cytokine production. J. Immuno 194(9), 4498-4506. https://doi.org/10.4049/jimmunol.1401399

Kimelman, D., and Xu, W. (2006). $\beta$-Catenin destruction complex: insights and questions from a structural perspective. Oncogene, 25, 7482-7491. https://doi.org/10.1038/sj.onc.1210055.

Klaassen, C. D., Liu, J., and Diwan, B. A. (2009). Metallothionein protection of cadmium toxicity. Toxicol. Appl. Pharmacol,238(3), 215-220. https://doi.org/10.1016/j.taap.2009.03.026.

Komiya, Y., and Habas, R. (2008). WNT signal transduction pathways. Organogenesis, 4(2), 68-75. https://doi.org/10.4161/org.4.2.5851.

Koumenis, C., and Wouters, B.G. (2006). "Translating" tumor hypoxia: unfolded protein response (UPR)-dependent and UPR-independent pathways. Mol Cancer Res, 4(7), 42336. doi: 10.1158/1541-7786.MCR-06-0150.

Koumenis, C. (2006). ER stress, hypoxia tolerance andtumor progression. Curr Mol Med, 6, 55-69. https://doi.org/10.2174/156652406775574604. 
Korinek, V., Barker, N., Morin, P.J., van Wichen, D., de Weger, R., Kinzler, K.W., Vogelstein, B., and Clevers, H. (1997). Constitutive transcriptional activation by a betacatenin-Tcf complex in APC-/- colon carcinoma. Science, 275(5307), 1784-1787.

doi: $10.1126 /$ science.275.5307.1784.

Koritzinsky, M., Levitin, F., van den Beucken, T., Rumantir, R. A., Harding, N. J., Chu, K. C., Boutros, P. C., Braakman, I., and Wouters, B. G. (2013). Two phases of disulfide bond formation have differing requirements for oxygen. J Cell Biol., 203(4), 615627. https://doi.org/10.1083/jcb.201307185.

Kühl, M., Sheldahl, L.C., Park, M., Miller, J.R., and Moon, R.T. (2000). The WNT/Ca ${ }^{2+}$ pathway: a new vertebrate WNT signaling pathway takes shape. Trends Genet, 16(7), 279-83. https://doi.org/10.1016/s0168-9525(00)02028-x

Kurayoshi, M., Yamamoto, H., Izumi, S., and Kikuchi, A. (2007). Post-translational palmitoylation and glycosylation of WNT-5a are necessary for its signalling. Biochem $J$, 402(3), 515-523. https://doi.org/10.1042/BJ20061476. 
Langer, R., Feith, M., Siewert, J.R., Hans-Juergen, W., and Heinz H. (2008). Expression and clinical significance of Glucose Regulated Proteins GRP78 (BiP) and GRP94 (GP96) in human adenocarcinomas of the esophagus. BMC Cancer, 70, 1-9. https://doi.org/10.1186/1471-2407-8-70.

Langerholc, T., Maragkoudakis, P. A., Wollgast, J., Gradisnik, L., \& Cencic, A. (2011). Novel and established intestinal cell line models - An indispensable tool in food science and nutrition. Trends Food Sci Technol, 22, S11-S20. https://doi.org/10.1016/j.tifs.2011.03.010.

Lee, A. H., Iwakoshi, N. N., and Glimcher, L. H. (2003). XBP-1 regulates a subset of endoplasmic reticulum resident chaperone genes in the unfolded protein response. Mol Cell.Biol, 23(21), 7448-7459. https://doi.org/10.1128/MCB.23.21.7448-7459.2003.

Lee, J. H., Yoon, Y. M., and Lee, S. H. (2017). Hypoxic Preconditioning Promotes the Bioactivities of Mesenchymal Stem Cells via the HIF-1 $\alpha$-GRP78-Akt Axis. Int. J. Mol Sci, 18(6), 1320. https://doi.org/10.3390/ijms18061320. 
Lee, M. A., Park, J. H., Rhyu, S. Y., Oh, S. T., Kang, W. K., and Kim, H. N. (2014). WNT3a expression is associated with MMP-9 expression in primary tumor and metastatic site in recurrent or stage IV colorectal cancer. BMC cancer, 14, 125. https://doi.org/10.1186/1471-2407-14-125.

Li, B., and Niswander, L. A. (2020). TMEM132A, a novel WNT signaling pathway regulator through WNTless (WLS) interaction. Front. Cell. Dev Biol, 8, 599890. https://doi.org/10.3389/fcell.2020.599890.

Li, Y., Lin, A. W., Zhang, X., Wang, Y., Wang, X., \& Goodrich, D. W. (2007). Cancer cells and normal cells differ in their requirements for Thoc1. Cancer Research, 67(14), 6657-6664. https://doi.org/10.1158/0008-5472.CAN-06-3234.

Li, Z., and Li, Z. (2012). Glucose regulated protein 78: a critical link between tumor microenvironment and cancer hallmarks. Biochim Biophys Acta, 1826 (1),13-22. https://doi.org/10.1016/j.bbcan.2012.02.001.

Lin, J. H., Walter, P., and Yen, T. S. (2008). Endoplasmic reticulum stress in disease pathogenesis. Anmu Rev Pathol,3,399-425. https://doi.org/10.1146/annurev.pathmechdis.3.121806.151434. 
Linder, M.E. (2001). Reversible modification of proteins with thioester-linked fatty acids. Enzymes, 21, 215 - 240. https://doi.org/10.1016/S1874-6047(01)80021-4.

Liu, F., Inageda, K., Nishitai, G., and Matsuoka, M. (2006). Cadmium induces the expression of Grp78, an endoplasmic reticulum molecular chaperone, in LLC-PK1 renal epithelial cells. Environ. Health Perspect, 114(6), 859-864. https://doi.org/10.1289/ehp.8920.

Ljungberg, J. K., Kling, J. C., Tran, T. T., \& Blumenthal, A. (2019). Functions of the WNT signaling network in shaping host responses to infection. Frontiers Immunol, 10, 2521. https://doi.org/10.3389/fimmu.2019.02521.

Logan, C.Y, and Nusse, R. (2004). The WNT signaling pathway in development and disease. Anmu. Rev. Cell Dev. Biol, 20, 781-810. https://oi.org/10.1146/annurev.cellbio.20.010403.113126.

Lv, Y., Wang, P.; Huang, R., Liang, X., Wang, P., Tan, J., Chen, Z., Dun, Z., Wang, J., Jiang, Q. Jiang, Q., Wu, S., Ling, H., Li, Z., Yang, X. 2017. Cadmium exposure and osteoporosis: A population-based study and benchmark dose estimation in southern China. J Bone Miner Res, 32 (10), 1990-2000. doi: 10.1002/jbmr.3151. 
MacDonald, B. T., Tamai, K., and He, X. (2009). WNT/beta-catenin signaling: components, mechanisms, anddiseases. Dev cell, 17(1), 9-26. hittps://doi.org/10.1016/j.devcel.2009.06.016.

Main, A., and Fuller, W. (2021). Protein S-Palmitoylation: advances and challenges in studying a therapeutically important lipid modification. FEBS J doi: $10.1111 /$ febs. 15781 .

Margoshes, M., and Vallee, B.L. (1957). A cadmium protein from equine kidney cortex. Am. Chem. Soc, 79, 4813-4814. https://doi.org/10.1021/ja01574a064.

Mårtensson, C.U., Priesnitz, C., Song, J., Ellenrieder, L., Doan, K.N., Boos, F., Floerchinger, A., Zufall1, N., Oeljeklaus, S., Warscheid, B and Becke, B. (2019). Mitochondrial protein translocation-associated degradation. Nature, 569, 679-683. https://doi.org/10.1038/s41586-019-1227.

Mason, J. O., Kitajewski, J., and Varmus, H. E. (1992). Mutational analysis of mouse WNT-1 identifies two temperature-sensitive alleles and attributes of WNT-1 protein essential for transformation of a mammary cell line. Mol Biol Cell, 3(5), 521-533. https://doi.org/10.1091/mbc.3.5.521 
Menke, A., Muntner, P., Silbergeld, E. K., Platz, E. A., \& Guallar, E. (2009). Cadmium levels in urine and mortality among U.S. adults. Environ Health Perspect, 117(2), 190196. https://doi.org/10.1289/ehp.11236

Mosimann, C., Hausmann, G. and Basler, K. (2009). $\beta$-Catenin hits chromatin: regulation of WNT target gene activation. Nat Rev Mol Cell Biol, 10, 276-286. https://doi.org/10.1038/nrm2654.

Matano, M., Date, S., Shimokawa, M., Takano, A., Fujii, M., Ohta, Y., Watanabe, T., Kanai, T., and Sato, T. (2015). Modeling colorectal cancer using CRISPR-Cas9-mediated engineering of human intestinal organoids. Nat Med, 21(3), 256-62. doi: 10.1038/nm.3802.

McGarry, T., Biniecka, M., Veale, D.J., and Fearon, U. (2018). Hypoxia, oxidative stress and inflammation. Free Radic Biol Med, 125, 15-24. doi:10.1016/j.freeradbiomed.2018.03.042. 
Merenda, A., Fenderico, N., \& Maurice, M. M. (2020). Wnt Signaling in 3D: Recent advances in the applications of intestinal organoids. Trends in cell biology, 30(1), 60-73. https://doi.org/10.1016/j.tcb.2019.10.003.

Méplan, C., Mann, K., \& Hainaut, P. (1999). Cadmium induces conformational modifications of wildtype p53 and suppresses p53 response to DNA damage in cultured cells. JBiolChem274(44),31663-31670. https://doi.org/10.1074/jbc.274.44.31663

Metcalfe, C., and Bienz, M. (2011). Inhibition of GSK3 by WNT signalling - Two contrasting models. J. Cell Sci, 124 (21)3537-3544. https://doi.org/10.1242/jcs.091991.

Mihara, E., Hirai, H., Yamamoto, H., Tamura-Kawakami, K., Matano, M., Kikuchi, A., Sato, T., and Takagi, J. (2016). Active and water-soluble form of lipidated WNT protein is maintained by a serum glycoprotein afamin/a-albumin. eLife, 5, e11621. https://doi.org/10.7554/eLife.11621.

Mikels, A.J., and Nusse, R. (2006). WNTs as ligands: processing, secretion and reception, Oncogene, 25, 7461-7468. https://doi.org/10.1038/sj.onc.1210053. 
Miller, J.R., Hocking, A.M., Brown, J.D., \& Moon, R.T. (1999). Mechanism and function of signal transduction by the Wnt/beta-catenin and $\mathrm{Wnt} / \mathrm{Ca}_{2}{ }^{+}$pathways. Oncogene, 18(55), 7860-7872. https://doi.org/10.1038/sj.onc.1203245.

Mohamed, E., Cao, Y. and Rodriguez, P.C. (2017). Endoplasmic reticulum stress regulates tumor growth and anti-tumor immunity: a promising opportunity for cancer immunotherapy. Cancer Immunol Immunother, 66, 1069-1078. https://doi.org/10.1007/s00262-017-2019-6.

Moffatt, P., and Denizeau, F. (1997). Metallothionein in physiological and physiopathological processes. Drug Metab Rev, 9(1-2):261-307. doi: 10.3109/03602539709037585.

Moti, N., Yu, J., Boncompain, G., Perez, F., and Virshup, D. M. (2019). WNT traffic from endoplasmic reticulum to filopodia. PloS ONE, 14(2), e0212711. https://doi.org/10.1371/journal.pone.0212711.

Morin, P. J., Vogelstein, B., and Kinzler, K. W. (1996). Apoptosis and APC in colorectal tumorigenesis. $P N A S, 93(15), 7950-7954$. https://doi.org/10.1073/pnas.93.15.7950. 
Motohashi, H., O'Connor, T., Katsuoka, F., Engel, J.D., and Yamamoto, M. (2002). Integration and diversity of the regulatory network composed of Maf and CNC families of transcription factors. Gene, 294(1-2),1-12. doi: 10.1016/s0378-1119(02)00788-6.

Morin, P.J., Sparks, A.B., Korinek, V., Barker, N., Clevers, H., Vogelstein, B., and Kinzler, K.W. (1997). Activation of beta-catenin-Tcf signaling in colon cancer by mutations in betacatenin or APC. Science, 275(5307),1787-90. doi: 10.1126/science.275.5307.1787.

Mosimann, C., Hausmann, G. and Basler, K. (2009). $\beta$-Catenin hits chromatin: regulation of WNT target gene activation. Nat Rev Mol Cell Biol, 10, 276-286. https://doi.org/10.1038/nrm2654.

Mostafa, D.G., Khaleel, E.F., Badi, R.M., Abdel-Aleem, G.A., and Abdeen, H.M. 2019. Rutin hydrate inhibits apoptosis in the brains of cadmium chloride-treated rats via preserving the mitochondrial integrity and inhibiting endoplasmic reticulum stress. Neurol. Res, 41(7), 594-608. doi: 10.1080/01616412.2019.1596206. 
Muz, B., de la Puente, P., Azab, F., and Azab, A. K. (2015). The role of hypoxia in cancer progression, angiogenesis, metastasis, and resistance to therapy. Hypoxia, 3, 83-92. https://doi.org/10.2147/HP.S93413.

Nadanaka, S., Yoshida, H., Kano, F., Murata, M., and Mori, K. (2004). Activation of mammalian unfolded protein response is compatible with the quality control system operating in the endoplasmic reticulum. Mol Biol. Cell, 15(6), 2537-2548. https://doi.org/10.1091/mbc.e03-09-0693.

Nagao, A., Kobayashi, M., Koyasu, S., Chow, C., and Harada, H. (2019). HIF-1dependent reprogramming of glucose metabolic pathway of cancer cells and its therapeutic significance. Int JMol Sci, 20(2), 238. https://doi.org/10.3390/ijms20020238.

Nawaz, S., Klar, J., Wajid, M., Aslam, M., Tariq, M., Schuster, J., Baig, S. M., and Dahl, N. (2009). WNT10A missense mutation associated with a complete odonto-onychodermal dysplasia syndrome. Eur J Hum Genet, 17(12), 1600-1605. https://doi.org/10.1038/ejhg.2009.81. 
Niehrs, C., and Acebron, S. P. (2012). Mitotic and mitogenic WNT signalling. EMBO J, 31(12), 2705-2713. https://doi.org/10.1038/emboj.2012.12.

Nielsen, A. E., Bohr, A., and Penkowa, M. (2007). The balance between life and death of cells: roles of metallothioneins. Biomark. Insights, 1, 99-111. PMID: 19690641.

Niemann, S., Zhao, C., Pascu, F., Stahl, U., Aulepp, U., Niswander, L., Weber, J. L., and Müller, U. (2004). Homozygous WNT3 mutation causes tetra-amelia in a large consanguineous family. Am JHum Genet, 74(3), 558-563. https://doi.org/10.1086/382196.

Nishimura, T., Honda, H., and Takeichi, M. (2012). Planar cell polarity links axes of spatial dynamics in neural-tube closure. Cell, 149(5), 1084-97. doi: 10.1016/j.cell.2012.04.021.

Nouhi, Z., Chevillard, G., Derjuga, A., Blank, V. (2007). Endoplasmic reticulum association and $\mathrm{N}$-linked glycosylation of the human $\mathrm{Nrf3}$ transcription factor. FEBS Lett, 581(28), 5401-6. doi: 10.1016/j.febslet.2007.10.041. 
Novellasdemunt, L., Antas, P., and Li, V. S. (2015). Targeting WNT signaling in colorectal cancer. A review in the theme: cell signaling: proteins, pathways and mechanisms. Am. J. Physiol. Cell Physiol, 309(8), C511-C521. https://doi.org/10.1152/ajpcell.00117.2015.

Ntambi, J.M., and Miyazaki, M. (2003). Recent insights into stearoyl-CoA desaturase-1. Curr Opin Lipidol, 14(12), 255-261. doi: 10.1016/j.tem.2017.10.003.

Ntambi, J.M., and Miyazaki, M. (2004). Regulation of stearoyl-CoA desaturases and role in metabolism. Prog Lipid Res, 43(2), 91-104. doi: 10.1016/s0163-7827(03)00039-0.

Nusse, R. (2003). WNTs and Hedgehogs: lipid-modified proteins and similarities in signaling mechanisms at the cell surface. Dev, 130(22), 5297-305. doi: 10.1242/dev.00821.

Nusse, R. (2008). WNT signaling and stem cell control, Cell Res, 18, 523-527. https://doi.org/10.1038/cr.2008.47 
Nusse, R., and Varmus, H. (2012). Three decades of WNTs: a personal perspective on how a scientific field developed. EMBOJ, 31(12),2670-2684. https://doi.org/10.1038/emboj.2012.146.

Nusse, R., Fuerer, C., Ching, W., Harnish, K., Logan, C., Zeng, A., Ten Berge, D., and Kalani, Y. (2008). WNT signaling andstem cell control, Cold Spring Harb. Symp. Quant. Biol, 18,59-66. https://doi.org/10.1101/sqb.2008.73.035.

Nüsslein-Volhard, C., andWieschaus, E. (1980). Mutations affecting segment number and polarity in Drosophila. Nature 287, 795-801. https://doi.org/10.1038/287795a0.

Nüsslein-Volhard, C., Wieschaus, E., and Kluding, H. (1984). Mutations affecting the pattern of the larval cuticle in Drosophila melanogaster: I. Zygotic loci on the second chromosome. Wilehm Roux Arch Dev Biol,193(5), 267-282. doi: 10.1007/BF00848156.

Nygaard, R., Yu, J., Kim, J., Ross, D. R., Parisi, G., Clarke, O. B., Virshup, D. M., and Mancia, F. (2021). Structural basis of WLS/Evi-mediated WNT transport and Secretion. Cell, 184(1), 194-206.e14. https://doi.org/10.1016/j.cell.2020.11.038. 
Ohtsuji, M., Katsuoka, F., Kobayashi, A., Aburatani, H., Hayes, J. D., and Yamamoto, M. (2008). Nrf1 and Nrf2 play distinct roles in activation of antioxidant response element-dependent genes. $J$ Biol Chem, 283(48), 33554-33562. https://doi.org/10.1074/jbc.M804597200.

Oslowski, C. M., and Urano, F. (2011). Measuring ER stress and the unfolded protein response using mammalian tissue culture system. Meth Enzymol, 490, 71-92. https://doi.org/10.1016/B978-0-12-385114-7.00004-0.

Ozawa, M., Baribault, H., and Kemler, R. (1989). The cytoplasmic domain of the cell adhesion molecule uvomorulin associates with three independent proteins structurally related in different species. EMBO J, 8(6), 1711-1717.

Paiva, C. N., and Bozza, M. T. (2014). Are reactive oxygen species always detrimental to pathogens? ARS, 20(6), 1000-1037. https://doi.org/10.1089/ars.2013.5447.

Pearson, C.A., Prozialeck, W.C. (2001). E-Cadherin, $\beta$-catenin and cadmium carcinogenesis. Med. Hypotheses, 56, 573-581. https://doi.org/10.1054/mehy.2000.1243. 
Person, R. J., Tokar, E. J., Xu, Y., Orihuela, R., Ngalame, N. N., \& Waalkes, M. P. (2013). Chronic cadmium exposure in vitro induces cancer cell characteristics in human lung cells. Toxicol Appl Pharmacol, 273(2), 281-288. https://doi.org/10.1016/j.taap.2013.06.013

Pfaffenbach, K.T., and Lee, A.S. (2011). The critical role of GRP78 in physiologic andpathologic stress. Curr Opin Cell Biol, 23(2), 150-156. https:/doi.org/10.1016/j.ceb.2010.09.007.

Pfister, A.S., Kühl, M. (2018). Of WNTs and ribosomes. Prog Mol Biol Transl Sci, 153, 131-155. doi: 10.1016/bs.pmbts.2017.11.006.

Pino, M. S., and Chung, D. C. (2010). The chromosomal instability pathway in colon cancer. Gastroenterology, 138(6), 2059-2072. https://doi.org/10.1053/j.gastro.2009.12.065.

Polakis, P. (2000). WNT signaling and cancer. Genes Dev, 14(15), 1837-1851. PMID: 10921899.

Polakis, P. (2007). The many ways of WNT in cancer. Curr Opin Genet Dev, 17, 45-51. doi: 10.1016/j.gde.2006.12.007. 
Port, F., Hausmann, G., and Basler, K. (2011). A genome-wide RNA interference screen uncovers two p24 proteins as regulators of Wingless secretion. EMBO reports, 12(11), 1144-1152. https://doi.org/10.1038/embor.2011.165.

Proto, M. C., Fiore, D., Piscopo, C., Franceschelli, S., Bizzarro, V., Laezza, C., Lauro, G., Feoli, A., Tosco, A., Bifulco, G., Sbardella, G., Bifulco, M., \& Gazzerro, P. (2017). Inhibition of $\mathrm{Wnt} / \beta$-Catenin pathway and Histone acetyltransferase activity by Rimonabant: a therapeutic target for colon cancer. Scientific reports, 7(1), 11678. https://doi.org/10.1038/s41598-017-11688-x.

Prozialeck, W. C., and Edwards, J. R. (2012). Mechanisms of cadmium-induced proximal tubule injury: new insights with implications for biomonitoring and therapeutic interventions. J Pharmacol Exp Ther, 343(1), 2-12. https://doi.org/10.1124/jpet.110.166769.

Puertollano, M.A., Puertollano, E., de Cienfuegos, G.Á., and de Pablo, M.A. (2011). Dietary antioxidants: immunity and host defense. Curr Top Med Chem, 11(14), 1752-66. doi: $10.2174 / 156802611796235107$. 
Pukrop, T., Klemm, F., Hagemann, T., Gradl, D., Schulz, M., Siemes, S., Trümper, L., and Blinder, C. (2006). WNT 5a signaling is critical for macrophage-induced invasion of breast cancer cell lines. Proc Natl Acad Sci USA, 103, 5454-9. doi: 10.1073/pnas.0509703103.

Qu, J., Yue, L., Gao, J., and Yao, H. (2019). Perspectives on WNT signal pathway in the pathogenesis and therapeutics of chronic obstructive pulmonary disease. J Pharmacol Exp Ther, 369(3), 473-480. https://doi.org/10.1124/jpet.118.256222.

Rao, T.P., and Küh, I.M. (2010). An updated overview on WNT signaling pathways: a prelude for more. Circ Res, 106(12), 1798-806. doi: 10.1161/CIRCRESAHA.110.219840.

Riggleman, B., Schedl, P., and Wieschaus, E. (1990). Spatial expression of the Drosophila segment polarity gene armadillo is posttranscriptionally regulated by wingless. Cell, 63(3), 549-60. doi: 10.1016/0092-8674(90)90451-j.

Reily, C., Stewart, T. J., Renfrow, M. B., and Novak, J. (2019). Glycosylation in health and disease. Nat Rev Nephrol, 15(6), 346-366. https://doi.org/10.1038/s41581-019-01294. 
Rios-Esteves, J., and Resh, M. D. (2013). Stearoyl CoA desaturase is required to produce active, lipid-modified WNT proteins. Cell Reports, 4(6), 1072-1081. https://doi.org/10.1016/j.celrep.2013.08.027.

Rios-Esteves, J., Haugen, B., and Resh, M. D. (2014). Identification of key residues andregions important for porcupine-mediated WNT acylation. J Biol Chem, 289(24), 17009-17019. https://doi.org/10.1074/jbc.M114.561209.

Rouschop, K. M., Dubois, L. J., Keulers, T. G., van den Beucken, T., Lambin, P., Bussink, J., van der Kogel, A. J., Koritzinsky, M., and Wouters, B. G. (2013). PERK/eIF2 $\alpha$ signaling protects therapy resistant hypoxic cells through induction of glutathione synthesis and protection against ROS. Proc Natl Acad Sci USA 110(12), 46224627. https://doi.org/10.1073/pnas.1210633110.

Rubinfeld, B., Albert, I., Porfiri, E., Munemitsu, S., and Polakis, P. (1997). Loss of beta-catenin regulation by the APC tumor suppressor protein correlates with loss of structure due to common somatic mutations of the gene. Cancer Res, 57(20),4624-30. PMID: 9377578. 
Russell, J. O., and Monga, S. P. (2018). WNT/ $\beta$-catenin signaling in liver development, homeostasis and pathobiology. Annu Rev Pathol, 13, 351-378. https://doi.org/10.1146/annurev-pathol-020117-044010.

Sato, M., and Suzuki, S. (2007). Endoplasmic reticulum stress and metallothionein. Yakugaku Zasshi, 127(4), 703-8. Japanese. doi: 10.1248/yakushi.127.703.

Sano, R., and Reed, J. C. (2013). ER stress-induced cell death mechanisms. Biochim et Biophys Acta, 1833(12), 3460-3470. https://doi.org/10.1016/j.bbamcr.2013.06.028.

Schaale, K., Brandenburg, J., Kispert, A., Leitges, M., Ehlers, S., and Reiling, N. (2013). WNT6 is expressed in granulomatous lesions of Mycobacterium tuberculosisinfected mice andis involved in macrophage differentiation and proliferation. $J$ Immunol, 191, 5182-95. doi: 10.4049/jimmunol.1201819. 
Schatoff, E. M., Leach, B. I., and Dow, L. E. (2017). WNT signaling and colorectal cancer. Curr Colorectal Cancer Rep, 13(2), 101-110. https://doi.org/10.1007/s11888-0170354-9.

Schutte, R., Nawrot, T.S., Richart, T., Thijs, L., Vanderschueren, D., Kuznetsova, T., Van Hecke, E., Roels, H.A., and Staessen, J.A. (2008). Bone resorption and environmental exposure to cadmium in women: a population study. Environ. Health Perspect, 116(6), 777-783. https://doi.org/10.1289/ehp.11167.

Schwarz-Romond, T., Metcalfe, C., and Bienz, M. (2007) Dynamic recruitment of axin by Dishevelled protein assemblies. J. Cell Sci, 14, 484-492. https://doi.org/10.1242/jcs.002956.

Sekine, S., Shibata, T., Sakamoto, M., and Hirohashi, S. (2002). Target disruption of the mutant beta-catenin gene in colon cancer cell line HCT116: preservation of its malignant phenotype. $\quad$ Oncogene, 21(38), 5906-5911. https://doi.org/10.1038/sj.onc. 1205756.

Semenza G. L. (2012). Hypoxia-inducible factors in physiology and medicine. Cell, 148(3), 399-408. https://doi.org/10.1016/j.cell.2012.01.021. 
Sen, M., Lauterbach, K., El-Gabalawy, H., Firestein, G.S., Corr, M., and Carson, D.A. (2000). Expression and function of wingless and frizzled homologs in rheumatoid arthritis. Proc Natl Acad Sci USA, 97, 2791-6. doi: 10.1073/pnas.050574297.

Song, N. H., and Koh, J. W. (2012). Effects of cadmium chloride on the cultured human lens epithelial cells. Mol Vis, 18, 983-988.

Shen, M., Wang, L., Guo, X., Xue, Q., Huo, C., Li, X., Fan, L., and Wang, X. (2015). A novel endoplasmic reticulum stress-induced apoptosis model using tunicamycin in primary cultured neonatal rat cardiomyocytes. Mol Med Rep, 12(4), 5149-54. doi: 10.3892/mmr.2015.4040.

Shi, Y., Vattem, K. M., Sood, R., An, J., Liang, J., Stramm, L., and Wek, R. C. (1998). Identification and characterization of pancreatic eukaryotic initiation factor 2 alpha-subunit kinase, PEK, involved in translational control. Mol Cell Biol, 18(12), 7499-7509. https://doi.org/10.1128/MCB.18.12.7499. 
Shapiro, L., \& Weis, W. I. (2009). Structure and biochemistry of cadherins and catenins. Cold Spring Harbor Perspectives in Biology, 1(3), a003053. https://doi.org/10.1101/cshperspect.a003053.

Shin, D.H., Li, S.H., Chun, Y.S., Huang, L.E., Kim, M.S., and Park, J.W. (2008). CITED2 mediates the paradoxical responses of HIF-1 alpha to proteasome inhibition. Oncogene, 27(13), 1939-44. doi: 10.1038/sj.onc.1210826.

Spinelli, M., Fusco, S., and Grassi, C. (2018). Nutrient-dependent changes of protein palmitoylation: impact on nuclear enzymes and regulation of gene expression. Int $J \mathrm{Mol}$ Sci, 19(12), 3820. https://doi.org/10.3390/ijms19123820.

\section{Staessen, J.A., Roels, H.A., Emelianov, D., Kuznetsova, T., Thijs, L., Vangronsveld,} J., and Fagard, R. (1999). Environmental exposure to cadmium, forearm bone density, and risk of fractures: Prospective population study. Public health and environmental exposure to cadmium (pheecad) study group. Lancet, 353 (9159), 1140-1144. doi: 10.1016/s0140-6736(98)09356-8. 
Stewart, D., Killeen, E., Naquin, R., Alam, S., and Alam, J. (2003). Degradation of transcription factor Nrf2 via the ubiquitin-proteasome pathway and stabilization by cadmium. J Biol Chem, 278(4), 2396-402. doi: 10.1074/jbc.M209195200.

Stone, W. L., Krishnan, K., Campbell, S. E., and Palau, V. E. (2014). The role of antioxidants and pro-oxidants in colon cancer. World J Gastrointest Oncol, 6(3), 55-66. https://doi.org/10.4251/wjgo.v6.i3.55.

Strowitzki, M. J., Cummins, E. P., and Taylor, C. T. (2019). Protein hydroxylation by hypoxia-inducible factor (HIF) hydroxylases: Unique or ubiquitous? Cells, 8(5), 384. https://doi.org/10.3390/cells8050384.

Strutt, D. (2003). Frizzled signalling and cell polarisation in Drosophila and vertebrates. Development, 130(19), 4501-4513. doi: 10.1242/dev.00695.

Suryawanshi, A., Tadagavadi, R. K., Swafford, D., and Manicassamy, S. (2016). Modulation of inflammatory responses by WNT/ $\beta$-catenin signaling in dendritic cells: A novel immunotherapy target for autoimmunity and cancer. Front Immunol, 7, 460. https://doi.org/10.3389/fimmu.2016.00460. 
Swindle, E.J., and Metcalfe, D.D. (2007). The role of reactive oxygen species and nitric oxide in mast cell-dependent inflammatory processes. Immunol Rev, 217, 186-205.

Szatrowski, T.P, and Nathan, C.F. (1991). Production of large amounts of hydrogen peroxide by human tumor cells. Cancer Res, 51(3), 794-8. PMID: 1846317.

Tabaczar, S., Czogalla, A., Podkalicka, J., Biernatowska, A., and Sikorski, A. F. (2017). Protein palmitoylation: Palmitoyltransferases and their specificity. EBM 242(11), 1150-1157. https://doi.org/10.1177/1535370217707732.

Tabas, I., and Ron, D. (2011). Integrating the mechanisms of apoptosis induced by endoplasmic reticulum stress. Nature Cell Biol, 13(3), 184-190. https://doi.org/10.1038/ncb0311-184.

Takada, R., Satomi, Y., Kurata, T., Ueno, N., Norioka, S., Kondoh, H., Takao, T., andTakada, S. (2006). Monounsaturated fatty acid modification of WNT protein: its role in WNT secretion. Dev Cell, 11(6), 791-801. doi: 10.1016/j.devcel.2006.10.003. 
Tanaka, K., Okabayashi, K., Asashima, M., Perrimon, N., and Kadowaki, T. (2000).

The evolutionarily conserved porcupine gene family is involved in the processing of the WNT family. Eur J Biochem, 267(13),4300-11. doi: 10.1046/j.1432-1033.2000.01478.x.

Tang, X., Wu, Y., Belenkaya, T. Y., Huang, Q., Ray, L., Qu, J., and Lin, X. (2012).

Roles of N-glycosylation and lipidation in Wg secretion and signaling. Dev Biol, 364(1), 32-41. https://doi.org/10.1016/j.ydbio.2012.01.009.

Teh, M.T., Blaydon, D., Ghali, L.R., Briggs, V., Edmunds, S., Pantazi, E., Barnes, M.R., Leigh, I.M., Kelsell, D.P., and Philpott, M.P. (2007). Role for WNT16B in human epidermal keratinocyte proliferation and differentiation. J Cell Sci, 120(Pt 2), 330-9. doi: 10.1242/jcs.03329.

Templeton, D.M., and Liu, Y. (2010). Multiple roles of cadmium in cell death and survival. Chem Biol Interact, 188(2), 267-75. doi: 10.1016/j.cbi.2010.03.040.

Tetsu, O., and McCormick, F. (1999). Beta-catenin regulates expression of cyclin D1 in colon carcinoma cells. Nature, 398(6726), 422-6.doi: 10.1038/18884. 
Thevenod, F., and Chakraborty, P.K. (2010). The role of WNT/beta-catenin signaling in renal carcinogenesis: Lessons from cadmium toxicity studies, Curr Mol Med, 10 (4), 387-398. doi: $10.2174 / 156652410791316986$.

Thévenod, F., and Lee, WK. (2013). Cadmium and cellular signaling cascades: interactions between cell death and survival pathways. Arch Toxicol, 87(10), 1743-1786. doi: 10.1007/s00204-013-1110-9.

Thomlinson, R. H., and Gray, L. H. (1955). The histological structure of some human lung cancers and the possible implications for radiotherapy. Br J Cancer, 9(4), 539-549. https://doi.org/10.1038/bjc.1955.55.

Thorpe, C.J., Schlesinger, A., Carter, J.C., and Bowerman, B. (1997). WNT signaling polarizes an early C. elegans blastomere to distinguish endoderm from mesoderm. Cell, 90(4), 695-705. doi: 10.1016/s0092-8674(00)80530-9. 
Touma, M., Kang, X., Gao, F., Zhao, Y., Cass, A. A., Biniwale, R., Xiao, X., Eghbali, M., Coppola, G., Reemtsen, B., and Wang, Y. (2017). WNT11 regulates cardiac chamber development and disease during perinatal maturation. JCI. Insight, 2(17), e94904. https://doi.org/10.1172/jci.insight.94904.

Tu, B.P., Ho-Schleyer, S.C., Travers, K.J., and Weissman, J.S. (2000). Biochemical basis of oxidative protein folding in the endoplasmic reticulum. Science, 290 (5496), 15714. doi: 10.1126/science.290.5496.1571.

Uhlén, M., Fagerberg, L., Hallström, B.M., Lindskog, C., Oksvold, P., Mardinoglu, A., Sivertsson, Å., Kampf, C., Sjöstedt, E., Asplund, A., Olsson, I., Edlund, K., Lundberg, E., Navani, S., Szigyarto, C.A., Odeberg, J., Djureinovic, D., Takanen, J.O., Hober, S., Alm, T., Edqvist, P.H., Berling, H., Tegel, H., Mulder, J., Rockberg, J., Nilsson, P., Schwenk, J.M., Hamsten, M., von Feilitzen, K., Forsberg, M., Persson, L., Johansson, F., Zwahlen, M,, von Heijne, G., Nielsen, J., and Pontén, F. (2015). Proteomics. Tissue-based map of the human proteome. Science, 347(6220), 1260419. doi: 10.1126/science. 1260419 .

Umar S. (2010). Intestinal stem cells. Curr Gastroenterol Rep, 12(5), 340-348. https://doi.org/10.1007/s11894-010-0130-3. 
Urra, H., Dufey, E., Avril, T., Chevet, E., and Hetz, C. (2016). Endoplasmic reticulum stress and the hallmarks of cancer. Trends in cancer, 2(5), 252-262. https://doi.org/10.1016/j.trecan.2016.03.007.

Valvezan, A. J., Zhang, F., Diehl, J. A., and Klein, P. S. (2012). Adenomatous polyposis coli (APC) regulates multiple signaling pathways by enhancing glycogen synthase kinase3 (GSK-3) activity. J Biol Chem, 287(6), 3823-3832. https://doi.org/10.1074/jbc.M111.323337.

van den Heuvel, M., Harryman-Samos, C., Klingensmith, J., Perrimon, N., and Nusse, R. (1993). Mutations in the segment polarity genes wingless and porcupine impair secretion of the wingless protein. EMBO J, 12(13), 5293-5302. PMID: 8262072.

van Amerongen, R., Mikels, A., and Nusse, R. (2008). Alternative WNT signaling is initiated by distinct receptors. Sci Signal, 1(35), re9. doi: 10.1126/scisignal.135re9. 
van Amerongen, R., Bowman, A.N., and Nusse, R. (2012). Developmental stage andtime dictate the fate of WNT/ $\beta$-catenin-responsive stem cells in the mammary gland. Cell Stem Cell, 11, 387-400. https://doi.org/10.1016/j.stem.2012.05.023.

van den Heuvel, M., Harryman-Samos, C., Klingensmith, J., Perrimon, N., and Nusse, R. (1993). Mutations in the segment polarity genes wingless and porcupine impair secretion of the wingless protein. EMBO J, 12(13), 5293-5302. PMID: 8262072.

van der Vliet, A., Tuinstra, T.J., and Bast, A. (1989). Modulation of oxidative stress in the gastrointestinal tract and effect on rat intestinal motility. Biochem Pharmacol, 38(17), 2807-18. doi: 10.1016/0006-2952(89)90435-8.

van Lidth de Jeude, J. F., Meijer, B. J., Wielenga, M., Spaan, C. N., Baan, B., Rosekrans, S. L., Meisner, S., Shen, Y. H., Lee, A. S., Paton, J. C., Paton, A. W., Muncan, V., van den Brink, G. R., \& Heijmans, J. (2017). Induction of endoplasmic reticulum stress by deletion of Grp78 depletes Apc mutant intestinal epithelial stem cells. Oncogene, 36(24), 3397-3405. https://doi.org/10.1038/onc.2016.326. 
Vasak, M. (2005). Advances in metallothionein structure and functions. J Trace Elem Med Biol, 19(1), 13-17. https://doi.org/10.1016/j.jtemb.2005.03.003.

Venugopal, R., and Jaiswal, A.K. (1998). Nrf2 and Nrf1 in association with Jun proteins regulate antioxidant response element-mediated expression and coordinated induction of genes encoding detoxifying enzymes. Oncogene, 17(24), 3145-3156. https://doi.org/10.1038/sj.onc.1202237.

Vermeulen, L., De Sousa, E.M.F., van der Heijden, M., Cameron, K., de Jong, J.H., Borovski, T., Tuynman, J.B., Todaro, M., Merz, C., Rodermond, H., Sprick, M.R., Kemper, K., Richel, D.J., Stassi, G., and Medema, J.P. (2010). WNT activity defines colon cancer stem cells and is regulated by the microenvironment. Nat Cell Biol ,12(5), 468-476. doi: 10.1038/ncb2048.

Verras, M., Papandreou, I., Lim, A. L., and Denko, N. C. (2008). Tumor hypoxia blocks WNT processing and secretion through the induction of endoplasmic reticulum stress. Mol Cell Biol, 28(23), 7212-7224. https://doi.org/10.1128/MCB.00947-08. 
Very, N., Lefebvre, T., and El Yazidi-Belkoura, I. (2017). Drug resistance related to aberrant glycosylation in colorectal cancer. Oncotarget, 9(1), 1380-1402. https://doi.org/10.18632/oncotarget.22377.

Vincent, K.A., Shyu, K.G., Luo, Y., Magner, M., Tio, R.A., Jiang, C., Goldberg, M.A., Akita, G.Y., Gregory, R.J., and Isner, J.M. (2000). Angiogenesis is induced in a rabbit model of hindlimb ischemia by naked DNA encoding an HIF-1alpha/VP16 hybrid transcription factor. Circulation, 102(18), 2255-61. doi: 10.1161/01.cir.102.18.2255.

Von Zglinicki, T., Edwall, C., Ostlund, E., Lind, B., Nordberg, M., Ringertz, N.R., and Wroblewski, J. 1992. Very low cadmium concentrations stimulate DNA synthesis and cell growth. J Cell Sci, 103 (Pt 4), 1073-1081. PMID: 1487490.

Waisberg, M., Joseph, P., Hale, B., and Beyersmann, D. (2003). Molecular and cellular mechanisms of cadmium carcinogenesis. Toxicology, 192(2-3), 95-117. doi: $10.1016 / \mathrm{s} 0300-483 \times(03) 00305-6$.

Wang, G.L., and Semenza, G.L. (1995). Purification and characterization of hypoxiainducible factor 1. J Biol Chem, 270(3), 1230-7. doi: 10.1074/jbc.270.3.1230. 
Wang, X., Reid Sutton, V., Peraza-Llanes, O.J., Yu, Z., Rosetta, R., Kou, Y.C., Eble, T.N., Patel, A., Thaller, C., Fang, P., and Van Den Veyver, I.P. (2007). Mutations in Xlinked PORCN, a putative regulator of WNT signaling, cause focal dermal hypoplasia. Nat. Genet, 39, 836-838. https://doi.org/10.1038/ng2057.

Wang, M., Wey, S., Zhang, Y., Ye, R., and Lee, A. S. (2009). Role of the unfolded protein response regulator GRP78/BiP in development, cancer, and neurological disorders. $A R S$, 11(9), 2307-2316. https://doi.org/10.1089/ars.2009.2485.

Wang, H., Zhang, S. X., and Hartnett, M. E. (2013). Signaling pathways triggered by oxidative stress that mediate features of severe retinopathy of prematurity. JAMA Ophthalmol, 131(1), 80-85. https://doi.org/10.1001/jamaophthalmol.2013.986.

Wang, L., Zeng, X., Ryoo, H. D., and Jasper, H. (2014). Integration of UPRER and oxidative stress signaling in the control of intestinal stem cell proliferation. PLoS Genetics, 10(8), e1004568. https://doi.org/10.1371/journal.pgen.1004568. 
Wang, L. T., Lin, M. H., Liu, K. Y., Chiou, S. S., Wang, S. N., Chai, C. Y., Tseng, L. W., Chiou, H. C., Wang, H. C., Yokoyama, K. K., Hsu, S. H., and Huang, S. K. (2021). WLS/Wntless is essential in controlling dendritic cell homeostasis via a WNT signalingindependent mechanism. Autophagy, 1-16. Advance online publication. https://doi.org/10.1080/15548627.2021.1907516.

Wang, R., Zhang, P., Li, J., Guan, H., and Shi, G. (2016). Ubiquitination is absolutely required for the degradation of hypoxia-inducible factor-1 alpha protein in hypoxic conditions. Biochem Biophys Res Commun, 470(1), 117-122. doi: 10.1016/j.bbrc.2016.01.005.

Weber, C.E., Kuo, P.C. (2012). The tumor microenvironment. Surg Oncol, 21(3),172177. doi: 10.1016/j.suronc.2011.09.001.

Weidemann, A., and Johnson, R. (2008). Biology of HIF-1 $\alpha$. Cell Death Differ, 15, 621627. https://doi.org/10.1038/cdd.2008.12.

Wend, P., Wend, K., Krum, S.A., and Miranda-Carboni, G.A. (2012). The role of WNT10B in physiology and disease. Acta Physiol, 204(1), 34-51. doi: 10.1111/j.17481716.2011.02296.x. 
Willert, K., Brown, J.D., Danenberg, E., Duncan, A.W., Weissman, I.L., Reya, T., Yates, J.R., and Nusse, R. (2003). WNT proteins are lipid-modified and can act as stem cell growth factors. Nature, 423, 448-452. https://doi.org/10.1038/nature01611.

Weissenböck, M., Latham, R., Nishita, M., Wolff, L. I., Ho, H. H., Minami, Y., and Hartmann, C. (2019). Genetic interactions between Ror2 and WNT9a, Ror1 and WNT9a and Ror2 and Ror1: Phenotypic analysis of the limb skeleton and palate in compound mutants. Genes to cells, 24(4), 307-317. https://doi.org/10.1111/gtc.12676.

Wodarz, A., and Nusse, R. (1998). Mechanisms of WNT signaling in development. Annu Rev Cell Dev Biol, 14,59-88. doi: 10.1146/annurev.cellbio.14.1.59.

Wu, C. H., \& Nusse, R. (2002). Ligand receptor interactions in the Wnt signaling pathway in Drosophila. The Journal of biological chemistry, 277(44), 41762-41769. https://doi.org/10.1074/jbc.M207850200 
Xu, M., Horrell, J., Snitow, M., Cui, J., Gochnauer, H., Syrett, C. M., Kallish, S., Seykora, J. T., Liu, F., Gaillard, D., Katz, J. P., Kaestner, K. H., Levin, B., Mansfield, C., Douglas, J. E., Cowart, B. J., Tordoff, M., Liu, F., Zhu, X., Barlow, L. A., Rubin, A.I,, McGrath, J.A., Morrisey, E.E., Chu, E.Y., and Millar, S. E. (2017). WNT10A mutation causes ectodermal dysplasia by impairing progenitor cell proliferation and KLF4mediated differentiation. Nat Commun, 8, 15397. https://doi.org/10.1038/ncomms15397.

World Health Organization. (1992). Environmental Health Criteria 134: Cadmium. 1st ed. World Health Organization; Geneva, Switzerland.

Yang, Y., Topol, L., Lee, H., and Wu, J. (2003). WNT5a and WNT5b exhibit distinct activities in coordinating chondrocyte proliferation and differentiation. Dev, 130(5),100315. doi: $10.1242 / \mathrm{dev} .00324$.

Yao, K., Gietema, J. A., Shida, S., Selvakumaran, M., Fonrose, X., Haas, N. B., Testa, J., \& O'Dwyer, P. J. (2005). In vitro hypoxia-conditioned colon cancer cell lines derived from HCT116 and HT29 exhibit altered apoptosis susceptibility and a more angiogenic profile in vivo. BritishJCancer,93(12), 1356-1363. https://doi.org/10.1038/sj.bjc.6602864. 
Yoshida, H., Matsui, T., Yamamoto, A., Okada, T., and Mori, K. (2001). XBP1 mRNA is induced by ATF6 and spliced by IRE1 in response to ER stress to produce a highly active transcription factor. Cell, 107(7), 881-91. doi: 10.1016/s0092-8674(01)00611-0.

Yost, C., Torres, M., Miller, J.R., Huang, E., Kimelman, D., and Moon, R.T. (1996). The axisinducing activity, stability, andsubcellular distribution of beta-catenin is regulated in Xenopus embryos by glycogen synthase kinase 3. Genes Dev,10(12), 1443-1454. doi: 10.1101/gad.10.12.1443. PMID: 8666229.

Young, R. M., Ackerman, D., Quinn, Z. L., Mancuso, A., Gruber, M., Liu, L., Giannoukos, D. N., Bobrovnikova-Marjon, E., Diehl, J. A., Keith, B., and Simon, M. C. (2013). Dysregulated mTORC1 renders cells critically dependent on desaturated lipids for survival under tumor-like stress. Genes Dev, 27(10), 1115-1131. https://doi.org/10.1101/gad.198630.112.

Yue, Q., Wagstaff, L., Yang, X., Weijer, C., and Münsterberg, A. (2008). WNT3amediated chemorepulsion controls movement patterns of cardiac progenitors and requires RhoA function. Dev, 135(6), 1029-37. doi: 10.1242/dev.015321. 
Zeng, X., Huang, H., Tamai, K., Zhang, X., Harada, Y., Yokota, C., Almeida, K., Wang, J., Doble, B., Woodgett, J., Wynshaw-Boris, A., Hsieh, J. C., and He, X. (2008). Initiation of WNT signaling: control of WNT coreceptor Lrp6 phosphorylation/activation via Frizzled, Dishevelled and axin functions. Dev, 135(2), 367-375. https://doi.org/10.1242/dev.013540.

Zeng, Y. A., and Nusse, R. (2010). WNT proteins are self-renewal factors for mammary stem cells and promote their long-term expansion in culture. Cell stem cell, 6(6), 568-577. https://doi.org/10.1016/j.stem.2010.03.020.

Zhai, L., Chaturvedi, D., and Cumberledge, S. (2004). Drosophila WNT-1 undergoes a hydrophobic modification and is targeted to lipid rafts, a process that requires porcupine. $J$ Biol Chem, 279(32), 33220-7. doi: 10.1074/jbc.M403407200.

Zhan, T., Rindtorff, N. and Boutros, M. (2017). WNT signaling in cancer. Oncogene, 36, 1461-1473. https://doi.org/10.1038/onc.2016.304.

Zhang, K. (2010). Integration of ER stress, oxidative stress and the inflammatory response in health and disease. Int. J. Clin. Exp. Med, 3(1), 33-40. PMID: 20369038. 
Zhang, P., Wu, Y., Belenkaya, T. Y., and Lin, X. (2011). SNX3 controls Wingless/WNT secretion through regulating retromer-dependent recycling of WNTless. Cell Research, 21(12), 1677-1690. https://doi.org/10.1038/cr.2011.167.

Zhang, Y., Zu, D., Chen, Z., and Ying, G. (2020). An update on WNT signaling pathway in cancer. Transl Cancer Res, 9, 1246-1252. https://doi.org/10.21037/tcr.2019.12.50.

Zhdanov, A. V., Okkelman, I. A., Collins, F. W., Melgar, S., \& Papkovsky, D. B. (2015). A novel effect of DMOG on cell metabolism: direct inhibition of mitochondrial function precedes HIF target gene expression. Biochim et biophys Acta, 1847(10), 1254 1266. https://doi.org/10.1016/j.bbabio.2015.06.016

Zhu, Y. P., Zheng, Z., Hu, S., Ru, X., Fan, Z., Qiu, L., and Zhang, Y. (2019). Unification of opposites between two antioxidant transcription factors Nrf1 and Nrf2 in mediating distinct cellular responses to the endoplasmic reticulum stressor tunicamycin. Antioxidants, 9(1), 4. https://doi.org/10.3390/antiox9010004. 
Ziello, J. E., Jovin, I. S., and Huang, Y. (2007). Hypoxia-inducible factor (HIF)-1 regulatory pathway and its potential for therapeutic intervention in malignancy and ischemia. YJBM, 80(2), 51-60. PMID: 18160990.

Zimmermann, J., Erdmann, D., Lalande, I., Grossenbacher, R., Noorani, M., and Fürst, P. (2000). Proteasome inhibitor induced gene expression profiles reveal overexpression of transcriptional regulators ATF3, GADD153 and MAD1. Oncogene, 19(25), 2913-20. doi: 10.1038/sj.onc.1203606. 


\section{Appendices}

Table 2: List of all the primers used in all the experiments (Cloning, qPCR, EMSA,

siRNA).

\begin{tabular}{|c|c|}
\hline Experiment & Sequence \\
\hline Cloning & $\begin{array}{l}\text { Universal Sequencing Primers } \\
\text { T7 Promoter } \\
\text { 5'-TAATACGACTCACTATAG-3' } \\
\text { BGH Reverse } \\
\text { 5'-TAGAAGGCACAGTCGAGG-3' } \\
\text { PORCN } \\
\text { Forward: 5'-GGTCTCATATGCCACCTTTAGCCGCCAGGAATTTTTCC-3' } \\
\text { Reverse: 5'-ACAGAGAATTCTCAGCCTATGAGACGGTAGAAGATCCAG-3' } \\
\boldsymbol{W L S} \\
\text { Forward: 5'-GCGCACATATGGCTGGGGCAATTATAGAAAACATGAGC-3' } \\
\text { Reverse: 5'-GCTGCGAATTCCTACTCCTGGGCCTCCTTGCGGG-3' }\end{array}$ \\
\hline qPCR & $\begin{array}{l}\text { NFE2L1 } \\
\text { Forward: 5'-CTGGAGGAGGAATTTGACTCTG-3' } \\
\text { Reverse: 5-'GAGGAAGAGGAGGAGGAAGAA-3' } \\
\text { NFE2L2 } \\
\text { Forward: 5'-CCGGCATTTCACTAACACAAG-3' } \\
\text { Reverse: 5'-CAGAATCACTGAGGCCAAGTAG-3' } \\
\text { HIF1 } \boldsymbol{\alpha} \\
\text { Forward: 5'-CCAGTTACGTTCCTTCGATCAG-3' } \\
\text { Reverse: 5'-GTAGTGGTGGCATTAGCAGTAG-3' } \\
\text { GRP78 } \\
\text { Forward: 5'-CCTTCGATGTGTCTCTTCTCAC-3' } \\
\text { Reverse: 5'-ACGCTGGTCAAAGTCTTCTC-3' } \\
\text { PORCN } \\
\text { Forward: 5'-CTCCTTCCACTTCAGCAACTAT-3' } \\
\text { Reverse: 5'-CCATTCCAGGTGATCCTTCTC-3' } \\
\text { WLS } \\
\text { Forward: 5'-GGACATTGCCTTCAAGCTAAAC-3' } \\
\text { Reverse: 5'-CATTTCAGTCCACTCAGCAAAC-3' } \\
\text { WNT3A } \\
\text { Forward: 5'-GACTTCCTCAAGGACAAGTACG-3' } \\
\text { Reverse: 5'-GGAACCTTGAAGTAGGTGTAG-3' } \\
\text { WNT5A } \\
\text { Forward: 5'-CCCAGGACCCGCTTATTTATAG- 3' } \\
\text { Reverse: 5-'GGTTCCGGTTGCAATTCTTG-3' } \\
\text { MT1 } \\
\text { Forward: 5'-CTGGCTCCTGCAAATGCAAA- 3' } \\
\text { Reverse: 5'-GCACTTCTCTGATGCCCCTT-3' } \\
\text { GAPDH } \\
\text { Forward: 5'-ATCAAGAAGGTGGTGAAG-3' } \\
\text { Reverse: 5'-CCAAATTCGTTGTCATACC-3' }\end{array}$ \\
\hline EMSA & $\begin{array}{l}P O R C N \\
\text { Nrf-2 } \\
\text { 5'-Biotin-TGCTGGGGAATTAAGCATGCAAATCACTT-3' } \\
\text { 5'-AAGTGATTTGCATGCTTAATTCCCCAGCA-3' } \\
\text { HIF-1 } \\
\text { 5'-Biotin-ATTTAGAGGACGTGCGCGGGTGACTATT-3' } \\
\text { 5'-AATAGTCACCCGCGCACGTCCTCTAAAT-3' } \\
\text { NF-kB } \\
\text { 5'-Biotin-AATTACCATTGGTGTTTCCCAGCAAAAA-3' } \\
\text { 5'-TTTTTGCTGGGAAACACCAATGGTAATT-3' } \\
\text { WLS } \\
\text { Nrf-2 } \\
\text { 5'-Biotin-ATTAGAAATGAATGCTTCTATTAAGTGTA-3' } \\
\text { 5'-TACACTTAATAGAAGCATTCATTTCTAAT-3' } \\
\text { HIF-1 } \\
\text { 5'-Biotin-GTGGTAGAAACTGCACGTTTGGGGAGC-3' } \\
\text { 5'-GCTCCCCAAACGTGCAGTTTCTACCAC-3' }\end{array}$ \\
\hline si-RNA & $\begin{array}{l}\text { Porcupine C.D.Ri.13401.13.1 (PORCN) } \\
\text { Sense 5' rArArUrUrUrGrUrUrArUrUrGrUrCrArArArArUrA } \\
\text { Antisense 5' rUrArCrUrUrUrUrArUrUrUrGrArCrArArUrA } \\
\text { WNTless C.D.Ri.131398.13.7 (WLS) } \\
\text { Sense 5'rCrArArGrCrUrArArArCrArArCrCrArArU } \\
\text { Antisense 5'rUrUrCrUrCrUrGrArUrUrUrGrGrUrUrGrUrU } \\
\text { Negative Control siRNA }\end{array}$ \\
\hline
\end{tabular}


Sense 5' rCrGrUrUrArArUrCrGrCrGrUrArUrArArUrArCrGrCrGrUAT

Antisens5'rArUrArCrGrCrGrUrArUrUrArUrArCrGrCrGrGrArUrUrArArCrGrArC 


\section{List of Previous Publications}

Mohamed., R, Kennedy, C., Willmore, W. 2021. Responses of Porcupine and WNTless proteins to oxidative, hypoxic, and endoplasmic reticulum stresses, Cellular Signalling, 85:(1-12).10.1016/j.cellsig.2021.110047.

Mohamed., R and Willmore, W. 2021. Cadmium induced toxicity on WNT proteins and transcription factors. Toxicological Letters. (Submitted) reference number.

Mohamed., R and Willmore, W. 2021. Transcriptions factors activation implicated during WNT Secretion. Under revision

Mohamed, R., Groulx, E., Defilippi, S., Erak, T., Tambong, J.T., Tweddell, R.J., Tsopmo, A., Avis, T.J. 2017. Physiological and molecular characterization of compost bacteria antagonistic to soil-borne plant pathogens. Can J Microbiol. 2017, 63(5):411-426. doi: 10.1139/cjm-2016-0599.

DeFilippi, S., Groulx, E., Megalla, M., Mohamed, R., and Avis, T. J. 2018. Fungal competitors affect production of antimicrobial lipopeptides in Bacillus subtilis Strain B95. J. Chem. Ecol. 44, 374-383.

Kurniawan, O., Wilson, K., Mohamed, R., Avis, T.J. 2018. Bacillus and Pseudomonas spp. provide antifungal activity against gray mold and Alternaria rot on blueberry fruit, Biological Control, Volume 126, 36-141, https://doi.org/10.1016/j.biocontrol.2018.08.001. 


\section{Things I learned during my graduate studies}

1) Making new friends in other labs is extremely helpful (you get to use lots of machines $=$ more data) .

2) Read emails, and I mean literally all emails, you might miss on free stuff, I meant deadlines, and very important updates.

3) Apply for every scholarship as much as you can every year till you graduate

4) Volunteer its nicer to get involved you might be offered a job (trust me happens)

5) Be friendly and treat others nicely

6) Don't forget yourself in the process (enjoy life) be kind to yourself.

7) Attend conferences and talk (paid vacation all inclusive).

8) Say hello to your supervisor and ask them how they are doing they are humans and talk to them about other stuff not only data and orders from the science store

9) Attend departmental talks (free coffee, cookies and sometimes food). Of course, you will learn cool stuff from the presenter's research.

10) Read books other than scientific literature, be well informed about other things in life.

11) Its ok to complain about your experiments to your colleagues, they can help you sometimes by giving suggestions just don't over do it.

12) Keep at your desk the following (snacks, power bars, chocolate, candy, chips), they come handy when everything is closed.

13) Share funny science meme it does help easy up some of the boredom. 